\title{
Structure of Coset Models
}

\author{
Dissertation \\ zur Erlangung des Doktorgrades \\ der Mathematisch-Naturwissenschaftlichen Fakultäten \\ der Georg-August-Universität zu Göttingen
}

vorgelegt von

SÖREN KÖSTER

aus

Aachen

Göttingen 2003 
D 7

Referent: Prof. Dr. Karl-Henning Rehren 1

Korreferent: Prof. Dr. Detlev Buchholz 2

Tag der mündlichen Prüfung: 3. Juni 2003

\footnotetext{
${ }^{1}$ Institut für Theoretische Physik der Universität Göttingen

${ }^{2}$ Institut für Theoretische Physik der Universität Göttingen
} 


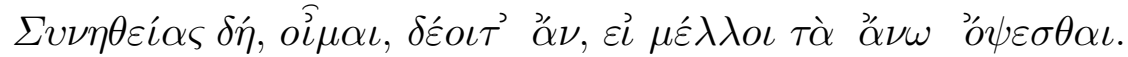
$\Pi о \lambda \iota \tau \varepsilon \iota \alpha \varsigma Z[\mathrm{P},(516 \mathrm{a} 4)]$. 
Zusammenfassung: Wir untersuchen Einbettungen lokaler, chiraler, konformer Quantentheorien $\mathcal{C} \subset \mathcal{B}$, die mit einer gegebenen Untertheorie $\mathcal{A} \subset \mathcal{B}$ vertauschen; die Untertheorien $\mathcal{C} \subset \mathcal{B}$ werden als Coset-Modelle bezeichnet. Die meisten Ergebnisse dieser Arbeit sind modellunabhängig, jedoch wird diese Untersuchung motiviert durch Einbettungen von Stromalgebren und deren CosETModelle.

Wir zeigen, dass es zu jeder gegebenen Untertheorie $\mathcal{A} \subset \mathcal{B}$ eine eindeutige, innere Darstellung $U^{\mathcal{A}}$ gibt, die die konforme Symmetrie auf der Untertheorie verwirklicht. Die lokalen beobachtbaren Größen von $\mathcal{B}$, die mit $U^{\mathcal{A}}$ vertauschen, bilden das maximale Coset-Modell $\mathcal{C}_{\max }$.

Unter der Annahme, dass $U^{\mathcal{A}}$ durch Integrale eines $\mathcal{A}$ innewohnenden Quantenfeldes erzeugt wird, zeigen wir: Die Einbettung der Untertheorie und ihrer Coset-Modelle steht in unmittelbarer Analogie zur Inklusion chiraler Theorien in einer 1+1-dimensionalen lokalen, konformen Quantentheorie. Die lokalen beobachtbaren Größen von $\mathcal{C}_{\max }$ zu einem bestimmten Gebiet sind gerade diejenigen Größen von $\mathcal{B}$, die mit den lokalen beobachtbaren Größen der Untertheorie $\mathcal{A}$ zu demselben Gebiet vertauschen.

Wir geben einige Anwendungen unserer Ergebnisse und diskutieren mögliche Verallgemeinerungen unserer Vorgehensweise.

Abstract: We study inclusions of local, chiral, conformal quantum theories $\mathcal{C} \subset \mathcal{B}$ which commute with a given subtheory $\mathcal{A} \subset \mathcal{B}$. These subtheories $\mathcal{C} \subset \mathcal{B}$ are called Coset models. Most of our results are model-independent, although our analysis is motivated by the inclusions of current algebras and their CosET models.

We prove that to every given $\mathcal{A} \subset \mathcal{B}$ there is a unique, inner representation $U^{\mathcal{A}}$ which implements conformal symmetry on the subnet. The local observables of $\mathcal{B}$ which commute with $U^{\mathcal{A}}$ form the maximal Coset model $\mathcal{C}_{\text {max }}$.

Assuming $U^{\mathcal{A}}$ to be generated by integrals of a quantum field affiliated with the subnet $\mathcal{A} \subset \mathcal{B}$, we show: The inclusion of the subnet and of its COSET models is directly analogous to the inclusion of chiral observables in a local, conformal theory in $1+1$ dimensions. The local observables of the maximal Coset model associated with a given region are found to be characterised by their commuting with the local observables of $\mathcal{A}$ associated with the very same region.

We give applications and discuss possible generalisations of our methods. 


\section{Contents}

\begin{tabular}{lll}
\hline I Introduction & 1
\end{tabular}

II $\quad$ Coset pairs of chiral subtheories $\quad 7$

1 Assumptions, conventions, and notions . . . . . . . . . 7

1.1 Chiral conformal symmetry . . . . . . . . . . . 7

1.2 Chiral nets, chiral subnets and their Coset models . . . . 9

1.3 Discussing the assumptions . . . . . . . . . . . . . . 12

2 Conformal transformations as observables . . . . . . . . . 22

2.1 BORCHERS-SUGAWARA construction . . . . . . . . . . 24

$2.2 \quad$ Direct applications to chiral subnets . . . . . . . . . . . 30

2.3 Discussion on the BORCHERS-SUGAWARA construction . . 34

III Subnets of chiral current algebras $\quad 36$

1 Quark models of chiral current algebras . . . . . . . . . . . . 37

1.1 Chiral currents as WiCk squares . . . . . . . . . . . 37

1.2 SUGAWARA construction for chiral current algebras . . . . 41

2 Chiral conformal nets of current algebras . . . . . . . . . . 44

2.1 Compact picture of chiral current algebras . . . . . . . 44

$2.2 \quad$ Integrating chiral current algebras . . . . . . . . . . . 46

$2.3 \quad$ From loop algebras to chiral current algebras . . . . . . . . 50

3 Current subalgebras as instructive examples . . . . . . . . . 53

4 Conformal covariance subalgebras . . . . . . . . . . . 56

$4.1 \quad$ Characterisation of conformal inclusions . . . . . . . 57

$4.2 \quad$ Covariant and invariant colours . . . . . . . . . . . 60

IV Local Nature of Coset Models $\quad 64$

1 Net-endomorphism Property . . . . . . . . . . . . . . 65

2 Chiral holography . . . . . . . . . . . . . . . . 70

2.1 The holographic quasi-theory in $1+1$ dimensions . . . . . . 71

$2.2 \quad$ Sharp geometrical action and time-like commutativity . . . 75

3 Solving the isotony problem . . . . . . . . . . . 78 
V Coset pairs of finite Index $\quad 82$

1 On local inclusions of Coset pairs . . . . . . . . . . . . . . . . . 82

2 Coset pairs as nets of subfactors . . . . . . . . . . . . . . . 87

2.1 The localised representation of a COSET pair . . . . . . . . 88

$2.2 \quad$ On spatial Coset pairs . . . . . . . . . . . . . . . . . . . 94

3 Coset construction of normal CTPS . . . . . . . . . . . . . . . 99

3.1 The Coset pairs of Goddard, Kent And Olive . . . . 101

3.2 Extension $\left(A_{10}, E_{6}\right)$ of $\mathcal{V} i r_{21}$ as a COSET model . . . . . 103

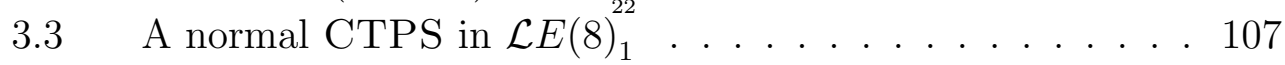

VI The conformally covariant derivatives of the $U(1)$ current 111

1 The chiral nets generated by the $\Phi^{(n)}$. . . . . . . . . . . . . . . . 112

$2 \quad$ No stress-energy tensor in $\Phi^{(n)}$-models . . . . . . . . . . . . . . 116

$\begin{array}{lr}\text { VII Discussion } & 118\end{array}$

$1 \quad$ Summary . . . . . . . . . . . . . . . . . . . . . . . . . . . . . . . . 118

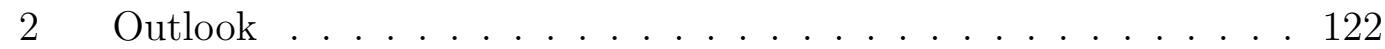

A Appendix 125

$1 \quad$ Lemmas on $\operatorname{PSL}(2, \mathbb{R})$ and $\operatorname{PSL}(2, \mathbb{R})^{\sim} \ldots \ldots$. . . . . . . . . 125

$2 \quad$ Lemmas on $\operatorname{Diff}_{+}\left(\mathrm{S}^{1}\right)$ and $\operatorname{Diff}_{+}\left(\mathrm{S}^{1}\right)^{\sim} \ldots \ldots$. . . . . . . . . . . . . . 128

3 Alternative argument for lemma IV.2 . . . . . . . . . . . . . . . . 130

4 Detailed proof of the BMT theorem . . . . . . . . . . . . . 131

$5 \quad$ Diff $_{+}\left(\mathrm{S}^{1}\right)$-Symmetry and $\operatorname{PSL}(2, \mathbb{R})$-Covariance . . . . . . . . . . . 132

Frequently used symbols $\quad 152$

\begin{tabular}{lc}
\hline Danksagungen & 153
\end{tabular} 


\section{Chapter I}

\section{Introduction}

The first quarter of the twentieth century saw two great revolutions is physics: the uncovering of quantum physics and the discovery of relativity. The quest for unifications of both in systems with infinitely many degrees of freedom, the development of relativistic quantum field theories, has led to remarkable successes, especially in elementary particle physics. The description of the physical laws valid in this context and our present understanding of the fundamental interactions, mainly summarised in the standard model for the strong, weak and electro-magnetic forces, has not yet reached the stage of a mathematically consistent theory. On the other hand, the underlying physical principles are clear:

The primary objects are expectation values of observable quantities. Not all observables are compatible, ie some sets of observables can not be measured simultaneously with arbitrary accuracy, like eg position and momentum due to HeisenBerG's uncertainty relation. The laws of physics are independent of the choice of frame of reference, ie they may be formulated covariantly with respect to the relativistic spacetime symmetry group. The principle of locality states that it is meaningful to talk of observables which can be measured in bounded spacetime regions and that observables are always compatible, if they are spacelike separated.

There are two concise mathematical frameworks which capture possible ways to formulate relativistic quantum field theories. In the first, one assigns to points $x^{\mu}$ in spacetime operator-valued distributions $\phi\left(x^{\mu}\right)$, the quantum fields, which may be viewed as quantised versions of the field strengths known from classical field theories. The principles of relativistic quantum field theory are reflected in certain requirements on the quantised fields, mostly known as Wightman's axioms [WG65]. Successes of this framework are summarised eg in [Jos65, SW64, Tod65, BLT75].

Another approach was proposed by HaAG AND Kastler [HK64]. Here, one selects regions $\mathcal{O}$ in spacetime and assigns to them (topological *-) algebras $\mathcal{A}(\mathcal{O})$ of bounded operators. The local algebras $\mathcal{A}(\mathcal{O})$ are regarded as the algebras of observations possible within the region $\mathcal{O}$ and their elements are called the local 
observables. The principles of relativistic quantum field theory can be expressed in this setting in a natural manner, leading to the framework of local quantum physics. This way of describing relativistic quantum field theory can be regarded as an extension of the picture of quantum fields and it has the advantage that one does not need to deal with particular sets of "coordinates" of the theory (the quantum fields). Especially structural and conceptual problems can be discussed within the setting of local quantum physics successfully (cf [Haa92, Buc00]).

The physics of a local quantum theory $\mathcal{A}$ is mainly encoded in the isotony inclusions of local algebras: if a region $\mathcal{O}_{1}$ is contained in another region $\mathcal{O}_{2}$ the property of isotony means that the local algebra $\mathcal{A}\left(\mathcal{O}_{1}\right)$ is contained in the algebra $\mathcal{A}\left(\mathcal{O}_{2}\right)$. Put differently: isotony says that the amount of possible observations increases with the localisation region they can be made in. This appears to be an almost trivial statement, but the isotony inclusions give structure of physical relevance to the local algebras which, ignoring this substructure, are (essentially) all isomorphic on quite general grounds [BDF87]. By isotony, a local quantum theory is a net of local algebras.

Within the framework of HAAG AND KASTLER it is natural to consider subtheories, ie inclusions of one local quantum theory, $\mathcal{A}$, in another one, $\mathcal{B}$, given by the inclusion of their local algebras: $\mathcal{A}(\mathcal{O}) \subset \mathcal{B}(\mathcal{O})$. The origin of these inclusions is encoded in two properties: they are covariant with respect to the action of the spacetime symmetry group and they are consistent with the net-structure of the subtheory, ie for $\mathcal{O}_{1} \subset \mathcal{O}_{2}$ we have: $\mathcal{A}\left(\mathcal{O}_{1}\right) \subset \mathcal{A}\left(\mathcal{O}_{2}\right)$.

Obviously, there are non-trivial subtheories like, for example, observables $\mathcal{A}$ included as gauge invariants in a local quantum theory $\mathcal{F}$ with a compact gauge symmetry. This example gives one motivation for studying subtheories: By the Doplicher-RoBERTS reconstruction [DR90] all the relevant information is in fact encoded in $\mathcal{A}$ already. This perspective is taken eg in [Ara92].

One might expect that the interaction between any subset of observables with the ambient system does not admit the subset to give rise to a local quantum theory essentially different from the whole theory. One could argue, for example, that local observables associated with energy and momentum already generate the whole net $\mathcal{A}$ and that the remaining freedom is taking local extensions like the local quantum theory $\mathcal{F}$ above (cf [Dop92, Con95]).

Actually, it seems that in most studies on subtheories $\mathcal{A} \subset \mathcal{B}$ the "energy content" of $\mathcal{B}$ is already contained in $\mathcal{A}$. In this work we are interested in cases where this is not the case. Rather, we want to look at the situation where the inclusion of $\mathcal{A}$ in $\mathcal{B}$ leaves enough space for other subtheories $\mathcal{C} \subset \mathcal{B}$ which commute with all of $\mathcal{A}$, ie the local subalgebras $\mathcal{C}(\mathcal{O})$ fulfill:

$$
\mathcal{C}(\mathcal{O}) \subset \mathcal{A}(\mathcal{O})^{\prime} \cap \mathcal{B}(\mathcal{O})=: \mathcal{C}_{\mathcal{O}}
$$

Such subtheories we call Coset models associated with $\mathcal{A} \subset \mathcal{B}$, and the algebras $\mathcal{C}_{\mathcal{O}}$ are called the local relative commutants. Admittedly quite trivial examples of 
this structure are tensor products of local quantum theories defined by $\mathcal{B}(\mathcal{O}):=$ $\mathcal{A}(\mathcal{O}) \otimes \mathcal{C}(\mathcal{O})$, but there are examples which are less simple.

We are interested in typical Coset models $\mathcal{C}$, in a description of the relative position of $\mathcal{A}, \mathcal{C}$ in the ambient theory $\mathcal{B}$, and in objects naturally associated with these inclusions. For example, one may ask immediately: Is there a maximal Coset model and how is it characterised? The local relative commutants $\mathcal{C}_{\mathcal{O}}$ are upper bounds for the local algebras of all CosET models, and if they define a Coset model themselves, then this is maximal, obviously.

As covariance is automatic, one has to show that the $\mathcal{C}_{\mathcal{O}}$ increase with $\mathcal{O}$. Since for $\mathcal{O}_{1} \subset \mathcal{O}_{2}$ we have $\mathcal{A}\left(\mathcal{O}_{1}\right)^{\prime} \supset \mathcal{A}\left(\mathcal{O}_{2}\right)^{\prime}$, we need arguments which ensure that this inclusion is inverted upon intersection with $\mathcal{B}\left(\mathcal{O}_{1}\right)$ and $\mathcal{B}\left(\mathcal{O}_{2}\right)$, respectively. For a general subtheory $\mathcal{A} \subset \mathcal{B}$ it is not obvious how to obtain such arguments, and this task will be referred to as the isotony problem.

Results of CARPI AND CONTI [CC01] indicate that in $3+1$ dimensions the structure of subnets essentially reduces to the tensor product scenario $\mathcal{A} \subset \mathcal{B}=$ $\mathcal{A} \otimes \mathcal{C}$ (under some additional assumptions). In these cases the isotony problem is absent and, in fact, it had not to be discussed explicitly in [CC01].

In two dimensions, however, we know of many subtheories $\mathcal{A} \subset \mathcal{B}$ which have interesting Coset models and a less simple relative position for the pair $\mathcal{A}, \mathcal{C}$ in $\mathcal{B}$. Characteristic for these examples is their high spacetime symmetry: they are not only covariant with respect to scale transformations $x^{\mu} \mapsto \lambda x^{\mu}$, but in fact covariant with respect to the whole stabiliser group of light-like directions, the conformal group. Conformal symmetry facilitates analysing such models a great deal. A physicist interested in relativistic quantum field theory has to ask for reasons justifying investigations in this setting.

The classical equations of motion of a typical massless field theory (containing no dimensional parameters), like MAXWELL's equations, are not only POINCARÉ and scale invariant, but actually invariant with respect to the whole conformal group. Initially, the hope was that in the high energy limit of a quantum field theory, where masses of particles do not make much of a difference, there holds an asymptotic conformal symmetry. The appearance of renormalisation scale dependent anomalous dimensions showed that scale symmetry (and a posteriori conformal symmetry) could not hold at the quantum level generally, but it could be valid at values of the coupling constant stable under the action of the renormalisation group (cf eg [FST89, Tod82] and references therein).

Renewed interest arose when Belavin, Polyakov and Zamolodchikov [BPZ84] took seriously the connection between conformally covariant quantum field theories in 1+1 dimensions and two-dimensional statistical systems undergoing a second order phase transition, like eg the critical IsING model, and exhibited how conformal symmetry in low dimensions allows one to obtain soluble models. And then, much of the work undertaken in string theory was made possible by the essential part that conformal symmetry in $1+1$ dimensions was able to play in this context as well (cf [GSW87]). 
From the perspective of local quantum physics there are some points of special interest: many of the soluble models can be shown to fit into this framework and thus they admit valuable case studies, which may teach us something about situations in four dimensions. Moreover, some new structures arise in this context, like non-trivial braid group statistics which is realised eg by quasi-particles in the fractional HALL effect. Both aspects led and lead to further insights into the structures of relativistic quantum field theory (cf eg [Tod01, Tod94]).

The models which inspire this work are generated by conserved currents in $1+1$ dimensions, $j^{a \mu}$. The currents transform in the adjoint representation of a compact, global gauge group, which is indicated by the label $a$. The LIE algebra $\mathfrak{g}$ of the gauge group determines, up to the normalisation of the current two-point functions, the commutation relations between the currents and hence the whole model, called the current algebra $\mathcal{A}_{\mathfrak{g}}$. By an inclusion of compact LIE algebras, $\mathfrak{h} \subset \mathfrak{g}$, one gets an inclusion of quantum field theories, the current subalgebra $\mathcal{A}_{\mathfrak{h}} \subset \mathcal{A}_{\mathfrak{g}}$. As many conformal fields in $1+1$ dimensions, the currents decompose into two commuting parts, each depending on one light cone coordinate, $t \pm x$, only; these generate the chiral current algebras. Hence, it suffices to look at the chiral inclusions only.

Infinitesimal coordinate transformations are implemented by a quadratic function of the currents, the stress-energy tensor $\Theta^{\mathfrak{g}}$. Since the current subalgebra possesses its own stress-energy tensor, $\Theta^{\mathfrak{h}}$, the field $\Theta^{\mathfrak{g}}-\Theta^{\mathfrak{h}}$ commutes with all of $\mathcal{A}_{\mathfrak{h}}$. $\Theta^{\mathfrak{g}}-\Theta^{\mathfrak{h}}$ is a stress-energy tensor itself, generically it does not vanish and hence generates a non-trivial CoseT model associated with the current subalgebra $\mathcal{A}_{\mathfrak{h}} \subset \mathcal{A}_{\mathfrak{g}}$. $\Theta^{\mathfrak{g}}-\Theta^{\mathfrak{h}}$ is called the CoseT stress-energy tensor and subtracting $\Theta^{\mathfrak{h}}$ from $\Theta^{\mathfrak{g}}$ is usually referred to as the Coset construction.

Goddard, Kent and Olive [GKO86] used the Coset construction to obtain the discrete series of chiral stress-energy tensors, which are of special interest due to a remarkable classification result [FQS86]. In fact, it proved possible to determine the features of the local quantum theories generated by these stress-energy tensors by studying the respective current algebras $\mathcal{A}_{\mathfrak{h}}, \mathcal{A}_{\mathfrak{g}}$ and the relative position of $\mathcal{A}_{\mathfrak{h}}$ in $\mathcal{A}_{\mathfrak{g}}$ [Lok94, KL02]. This was made possible, to a large extend, by works of XU ([Xu00a, Xu99] in particular), which are complementary to this work as they deal with particular inclusions of current algebras and their CosET models in the setting of local quantum physics.

More generally, one of the driving forces in the investigations on current subalgebras and COSET models was the interest in obtaining new models. A natural thing to do is to search for additional fields in the current algebra $\mathcal{A}_{\mathfrak{g}}$ which commute with all of $\mathcal{A}_{\mathfrak{h}}$, and to determine the algebra generated by these. This approach leads to $\mathcal{W}$-algebras. We will discuss COSET models from the perspective of local quantum physics and therefore we do not want to refer to particular field coordinates in COSET models. We will not comment further on the interesting achievements obtained on $\mathcal{W}$-algebras or on the related contributions to the understanding of current subalgebras and their COSET models from this side; we 
rather refer to [BS93, EHH93, Fuc97, Wat97] and references therein.

In the following, we want to broaden the perspective, to achieve clarity through a search for intrinsic structures and to shed new light on old problems. We intend to work in the spirit of the general theory of quantised fields, which "... analyzes the notions which are at the basis of all previously analyzed models", as RES Jost captured it [Jos65].

The next chapter provides a summary on chiral conformal quantum field theory in the sense of HAAG AND KASTLER. We will give a technical formulation of the questions raised in this introduction, state and discuss our main assumptions and obtain first, completely model independent results. In particular, we will see that for any chiral subtheory $\mathcal{A} \subset \mathcal{B}$ there is a unique implementation $U^{\mathcal{A}}$ of conformal transformations on $\mathcal{A}$ by unitary operators which are affiliated with $\mathcal{A}$ in a global sense; this inner-implementing representation $U^{\mathcal{A}}$ is the natural abstract counterpart of the stress-energy tensor of a current subalgebra. We prove that the local operators in $\mathcal{B}$ which commute with $U^{\mathcal{A}}$ form the maximal CoseT model $\mathcal{C}_{\text {max }}$ associated with $\mathcal{A} \subset \mathcal{B}$.

Thus, we are led to study subnets and their COSET models by analysing the action of the inner-implementing representation $U^{\mathcal{A}}$ of the subtheory $\mathcal{A}$ on the local observables of the ambient theory $\mathcal{B}$. This ansatz is new and the essence of this work. In particular, the isotony problem may be dealt with in a natural manner.

The chiral current subalgebras $\mathcal{A}_{\mathfrak{h}} \subset \mathcal{A}_{\mathfrak{g}}$ are discussed as examples satisfying our assumptions in chapter III. We give the relation between the inner implementation $U^{\mathcal{A}_{\mathfrak{h}}}$ of conformal transformations and the stress-energy tensor $\Theta^{\mathfrak{h}}$. This relation allows to establish some special properties of $U^{\mathcal{A}_{\mathfrak{h}}}$ (lemmas IV.2, IV.12 in chapter IV), which we believe to hold in general. We take the presence of a stress-energy tensor as the Additional Assumption for the subsequent analysis.

Chapter IV constitutes the main part of this work: It provides a solution of the isotony problem and thus establishes the identity of the local algebras of the maximal CoseT model $\mathcal{C}_{\max }$ and the local relative commutants of a chiral subtheory $\mathcal{A} \subset \mathcal{B}$. This way, we prove that the local operators in the ambient theory $\mathcal{B}$ which are contained in some CosET model may be characterised entirely in terms of local data, namely their commutativity with the respective local operators of the subtheory $\mathcal{A}$. Hence, all Coset models are of a local nature.

It is shown that through the action of $U^{\mathcal{A}}$ on the chiral theory $\mathcal{B}$ one may construct a $1+1$-dimensional quasi-theory $\mathcal{B}^{1+1}$ containing the original net $\mathcal{B}$ as "time zero subtheory"; this construction may be regarded as chiral holography. Taking $\mathcal{A}_{\text {max }}$ as the subtheory of $\mathcal{B}$ consisting of all local observables on which $U^{\mathcal{A}}$ implements the conformal symmetry, $\mathcal{B}^{1+1}$ contains $\mathcal{C}_{\max }$ and $\mathcal{A}_{\text {max }}$ as subtheories of chiral observables, each depending on one light cone coordinate in the holographic $1+1$-dimensional spacetime only. This establishes $\mathcal{A}_{\max }$ as a natural object connected with a subtheory $\mathcal{A} \subset \mathcal{B}$ and gives a straightforward interpretation for chiral subtheories and their COSET models as fixed-points with respect 
to a classical spacetime symmetry in a suitably enlarged ambient theory.

Chapter $\mathrm{V}$ is devoted to making contact with related works in the field. We spell out how the inclusion of a subtheory $\mathcal{A} \subset \mathcal{B}$ and its Coset models $\mathcal{C}$ may be seen as localised representations of the tensor product of the subtheories in the sense of Doplicher, HAAG AND RoBERTS. This formulation is used to get some more insights, mainly under the assumption that the localised representation has finite statistics. Moreover, we give a CosET construction for some normal canonical tensor product subfactors as introduced by REHREN [Reh00], when we revisit current subalgebras.

The limitations of the additional assumption on the presence of a stress-energy tensor are the issue of chapter VI: we prove that a class of models do not contain a stress-energy tensor. The concluding chapter briefly summarises the main results and gives an outlook on possible generalisations of the discussions given here. The appendix contains a few technical lemmas and miscellaneous results. 


\section{Chapter II}

\section{Coset pairs of chiral subtheories}

We introduce conformal quantum field theories in one chiral dimension, their subnets and the associated Coset models, which are the objects of this work. We discuss our main assumptions and obtain model independent results. In particular, section 2 is devoted to the construction of a representation $U^{\mathcal{A}}$ which implements chiral conformal symmetry on any given chiral subtheory $\mathcal{A} \subset \mathcal{B}$, is affiliated with $\mathcal{A}$ in a global sense and uniquely determined by these two properties. This representation forms the foundation of the subsequent analysis.

\section{Assumptions, conventions, and notions}

\subsection{Chiral conformal symmetry}

Conformal transformations of spacetime are required to leave the LORENTZ metric invariant up to a WeYL scaling. In $1+1$ dimensions, this group is infinitedimensional and contains localised diffeomorphisms. In positive-energy representations, only a finite-dimensional subgroup of this symmetry group remains unbroken, the group of global conformal transformations. If one restricts attention to this subgroup, the structures are similar to the situation of conformal symmetry in higher dimensions [BGL93]. When we speak of conformal symmetry in the following, we mean symmetry with respect to global conformal transformations.

Conformally covariant models are given as local nets on Minkowski space. In this context, conformal transformations have an action through mapping the observables associated with a given localisation region to the observables of the transformed region, as long as this is contained in Minkowski space. Following general arguments these nets may be extended to theories over the conformal covering of MinKOWSKI space, on which the conformal transformations act as a proper spacetime symmetry group. The extensions then form conformal quantum field theories. This scheme has been discussed for the general case by BRUNETTI, Guido And Longo [BGL93] (cf [Seg71], [LM75]) and for the chiral case by 
FREDENHAGEN AND JÖRSS [FJ96].

In $1+1$ dimensions, the light-cone (at the origin) consists of the two (chiral) light-rays, which provide the right and the left light-cone coordinates on MinkOWski space. Each conformal transformation in $1+1$ dimensions may be represented as a product of two commuting chiral conformal transformations, which for themselves act on one light-cone coordinate only.

Chiral conformal quantum field theories arise in 1+1-dimensional models as subsystems of observables which are invariant with respect to chiral coordinate transformations on the other light-cone coordinate. Examples of this structure are stress-energy tensors, the $U(1)$ current and its conformally covariant derivatives (cf chapters III and VI). In the following we restrict our attention to one chiral sector.

The conformal transformations of the chiral light-ray, $\mathbb{R}$, form a group isomorphic to $\operatorname{PSL}(2, \mathbb{R})$, which is the factor group of $S L(2, \mathbb{R})$ modulo the equivalence relation $A \sim-A$. Such a matrix $A=\left(\begin{array}{ll}a & b \\ c & d\end{array}\right)$ acts on the light-cone coordinate $x \in \mathbb{R}$ as follows:

$$
g_{\mathrm{A}} x=\frac{a x+b}{c x+d} .
$$

Some one-parameter subgroups of $\operatorname{PSL}(2, \mathbb{R})$ of particular interest are the translations, $T(a) x=x+a$, scale transformations, $D(t) x=e^{t} x$, often called dilatations, and special conformal transformations, $S(n) x=x /(1+n x)$.

A chiral conformal transformation maps exactly one point to $\infty$ and assumes a definite asymptotic value at $\pm \infty$, and $P S L(2, \mathbb{R})$ has its natural geometric action on the compactified light-ray $S^{1} \cong \mathbb{R} \cup\{\infty\}$. This compactification is achieved by means of a CAYLEY transformation:

$$
z=\frac{i x+1}{-i x+1} .
$$

When we speak of the light-ray, $\mathbb{R}$, as a subset of the conformal covering space, $\mathrm{S}^{1}$, then we mean the inclusion of the corresponding image under the CAYLEY transformation. The positive light-ray, $\mathbb{R}_{+}$, corresponds to the upper half-circle, $\mathrm{S}_{+}^{1}$, and $\mathbb{R}_{-}$to the lower half-circle, $\mathrm{S}_{-}^{1}$. The point $-1 \in \mathrm{S}^{1}$ corresponds to the point at infinity of $\mathbb{R}$, the point 1 is the image of the origin on the light-ray.

The CAYLEY transformation induces an isomorphism ${ }^{1} P S L(2, \mathbb{R}) \cong P S U(1,1)$. The latter is the factor group of the group $S U(1,1)$ consisting of complex matrices which have determinant 1 and leave invariant $\operatorname{diag}(+1,-1)$, divided by the relation $\mathrm{A} \sim-\mathrm{A}$. Elements of $S U(1,1)$ have the form $\mathrm{A}=\left(\begin{array}{c}\alpha \beta \\ \bar{\beta} \\ \bar{\alpha}\end{array}\right)$ with $|\alpha|^{2}-|\beta|^{2}=1$; their action on $z \in S^{1}$ reads:

$$
g_{\mathrm{A}} z=\frac{\alpha z+\beta}{\bar{\beta} z+\bar{\alpha}} .
$$

\footnotetext{
${ }^{1} P S L(2, \mathbb{R})$ is isomorphic to the proper, orthochronous LORENTZ group in $2+1$ dimensions, $S O(2,1)_{+}^{\uparrow}$, too.
} 
The one-parameter group of rigid conformal rotations acts by multiplication with a phase: $R(\varphi) z=e^{i \varphi} z, \varphi \in[-\pi, \pi[$.

Implementations of chiral conformal symmetry are given by unitary, strongly continuous representations of the universal covering group of $P S L(2, \mathbb{R})$, which we denote by $P S L(2, \mathbb{R})^{\sim}$. There is a local identification between one-parameter subgroups of $P S L(2, \mathbb{R})$ and of $P S L(2, \mathbb{R})^{\sim}$ by the identity of their LIE algebras. We denote the one-parameter subgroups in $P S L(2, \mathbb{R})^{\sim}$ corresponding to the subgroups of $P S L(2, \mathbb{R})$ introduced above by $\tilde{T}, \tilde{D}, \tilde{S}$, and $\tilde{R}$, respectively. The covering projection from $P S L(2, \mathbb{R})^{\sim}$ onto $P S L(2, \mathbb{R})$ will be written $\mathrm{p}$.

We adopt the physicists' convention on elements of the LIE algebra which allows us to use the same symbols for the generators of one-parameter subgroups in $\operatorname{PSL}(2, \mathbb{R})$, for the corresponding generators in $\operatorname{PSL}(2, \mathbb{R})^{\sim}$ and their self-adjoint representatives as generators of unitary one-parameter groups in a unitary, strongly continuous representation of $P S L(2, \mathbb{R})^{\sim}$. The generator of translations, the momentum operator, will be denoted by $P$, the generator of special conformal transformations by $K$, and the generator of rigid conformal rotations, the conformal Hamiltonian, by $L_{0}$. By the parametrisations above we have the identity

$$
2 L_{0}=P-K \text {. }
$$

$L_{0}, P$, and $-K$ all are positive operators in a representation, if one of them is positive (proposition II.8).

The localisation regions are open, non-dense intervals contained in the circle, called the proper intervals. A connected, open subset $I$ of $\mathrm{S}^{1}$ is a proper interval, denoted by $I \Subset \mathrm{S}^{1}$, if its causal complement $I^{\prime}:=\mathrm{S}^{1} \backslash \bar{I}$ is not the empty set. The action of the global conformal symmetry group on points in the circle as in (II.3) induces an action of $P S L(2, \mathbb{R})$ on the set of proper intervals. For $g \in P S L(2, \mathbb{R})$ the image of an interval $I \Subset \mathrm{S}^{1}$ under this action will be denoted $g I$.

Occasionally, we will encounter operators which are phases, for example cocycles of ray-representations. In order to distinguish the set of these phases from the chiral conformal covering space we will use the notation $\mathbb{C}_{1} \equiv\left\{z \in \mathbb{C},|z|^{2}=1\right\}$.

\subsection{Chiral nets, chiral subnets and their COSET models}

After having clarified the geometric situation, we now state the HAAG-KASTLER axioms of local quantum physics [HK64] in a form adequate for chiral conformal quantum field theory [GL96, FJ96, GF93]. 
Definition II.1: A chiral conformal theory (in short: chiral net ) $\mathcal{B}$ is given by a map assigning to each proper interval, $I \Subset \mathrm{S}^{1}$, a V.NEUMANN algebra, $\mathcal{B}(I)$, of bounded operators on a separable HILBERT space, $\mathscr{H}$, fulfilling the following properties:

i) Isotony: If $I_{1} \subset I_{2}$, we have $\mathcal{B}\left(I_{1}\right) \subset \mathcal{B}\left(I_{2}\right)$.

ii) Locality: For $I_{1} \subset I_{2}^{\prime}, \mathcal{B}\left(I_{1}\right)$ commutes with $\mathcal{B}\left(I_{2}\right)$, ie $\mathcal{B}\left(I_{1}\right) \subset \mathcal{B}\left(I_{2}\right)^{\prime}$.

iii) Covariance: There is a strongly continuous, unitary representation, $U$, of $P S L(2, \mathbb{R})$ on $\mathscr{H}$ such that the adjoint action $A d_{U(g)}, g \in P S L(2, \mathbb{R})$, defines for each $I \Subset \mathrm{S}^{1}$ an isomorphism $\alpha_{g} \uparrow \mathcal{B}(I)$ from $\mathcal{B}(I)$ onto $\mathcal{B}(g I) ; \alpha_{g}$ stands for the action of $A d_{U(g)}$ on the net of local algebras:

$$
A d_{U(g)} \mathcal{B}(I)=\alpha_{g}(\mathcal{B}(I))=\mathcal{B}(g I), I \Subset \mathrm{S}^{1} .
$$

iv) Positivity of energy: The spectrum of the momentum operator $P$ is positive in the representation $U$.

v) Vacuum: The space of U-invariant vectors is one-dimensional. We choose a unit vector, the vacuum $\Omega$, which is assumed to be cyclic for the V.NEUMANN algebra $\bigvee_{I \Subset \mathrm{S}^{1}} \mathcal{B}(I)$.

Remark: The set of proper intervals in $S^{1}$ is not directed with respect to the partial order defined by inclusion and thus is not a net in the proper sense of the word. Same holds true for the set of local algebras. It would be rigorous to call them precosheaves (cf eg [GL96]), but for three reasons we will use the term net for chiral conformal theories: They are completely determined by their restrictions to the light-ray, which are genuine nets of local algebras. We regard chiral conformal theories as models for general structures connected with local quantum theories in "realistic spacetimes", which are given by nets of local algebras. Finally, speaking of nets of local algebras is common practise in the literature on chiral conformal theories.

The fundamental object of this study is an inclusion of a chiral conformal theory $\mathcal{A}$ in a theory $\mathcal{B}$ as just introduced. We adopt the following definition [Lon01]:

Definition II.2: A chiral conformal subtheory (short: chiral subnet) $\mathcal{A}$ embedded in $\mathcal{B}$, written as $\mathcal{A} \subset \mathcal{B}$, is given by a map from the set of proper intervals to $\mathrm{V}$.NEUMANN algebras, $I \mapsto \mathcal{A}(I)$, with the following properties:

i) Inclusion: $\mathcal{A}(I) \subset \mathcal{B}(I)$ for $I \Subset \mathrm{S}^{1}$.

ii) Isotony: If $I_{1} \subset I_{2}$, then $\mathcal{A}\left(I_{1}\right) \subset \mathcal{A}\left(I_{2}\right)$.

iii) Covariance: For all $g \in P S L(2, \mathbb{R})$ and $I \Subset S^{1}$ we have: $\mathcal{A}(g I)=\alpha_{g}(\mathcal{A}(I))$. 
We say that $\mathcal{A}$ is non-trivial, if its local algebras are different from $\mathbb{C} \mathbb{1}$ and do not coincide with the local algebras of $\mathcal{B}$.

The goal of this work is to find and to establish typical features related with the following objects:

Definition II.3: Any chiral subnet $\mathcal{C} \subset \mathcal{B}$ is called a Coset model associated with a given chiral subnet $\mathcal{A} \subset \mathcal{B}$, if we have $\mathcal{C}(I) \subset \mathcal{A}(I)^{\prime}$ for one and hence for all $I \Subset \mathrm{S}^{1}$.

The chiral subnet defined by $\mathcal{A} \subseteq \mathcal{C}(I):=\mathcal{A}(I) \vee \mathcal{C}(I)$ is called a Coset pair. The local relative commutants $\mathcal{C}_{I}, I \Subset \mathrm{S}^{1}$, of $\mathcal{A} \subset \mathcal{B}$ are given by $\mathcal{C}_{I}:=$ $\mathcal{A}(I)^{\prime} \cap \mathcal{B}(I)$.

Obviously, the local relative commutants contain the local algebras of any Coset model:

$$
\mathcal{C}_{I}=\mathcal{A}(I)^{\prime} \cap \mathcal{B}(I) \supset \mathcal{C}(I), I \Subset \mathrm{S}^{1} .
$$

Note that, a priori, the local relative commutants $\mathcal{C}_{I}$ do not define a Coset model, because isotony is not known to hold; we refer to this as the isotony problem.

We have chosen the term "CosET model" rather than "Coset theory" since typically one constructs the COSET subnet from a specific chiral subnet and analyses its features in this setting rather than defining it abstractly.

A general, yet not obvious consequence of the assumptions is that there always is a representation $U^{\mathcal{A}}$ of $\operatorname{PSL}(2, \mathbb{R})^{\sim}$ which implements conformal covariance on the subtheory $\mathcal{A}$ and whose unitaries are globally inner in $\mathcal{A}$, ie the operators $U^{\mathcal{A}}(\tilde{g}), \tilde{g} \in P S L(2, \mathbb{R})^{\sim}$, are contained in the V.Neumann algebra $\bigvee_{I \Subset S^{1}} \mathcal{A}(I)$. With a slight abuse of notation, we denote the global algebra of the subnet $\mathcal{A} \subset \mathcal{B}$ by $\mathcal{A}:=\bigvee_{I \Subset S^{1}} \mathcal{A}(I)$. It contains all local observables of the subtheory $\mathcal{A}$ as well as all observables which are weak limits of local observables of $\mathcal{A} \subset \mathcal{B}$ but not local themselves; the latter we call genuine global observables.

$U^{\mathcal{A}}$ is constructed and some desirable properties of it are derived in section 2. There is shown, for example, that there is exactly on inner-implementing representation $U^{\mathcal{A}}$ for $\mathcal{A}$, put differently: $U^{\mathcal{A}}$ is the inner implementation of conformal symmetry for $\mathcal{A} \subset \mathcal{B}$. Furthermore, the operators $U^{\mathcal{A}}(\tilde{g}) \neq \mathbb{1}$ are genuine global observables in $\mathcal{A}$. We have the following important consequence:

Lemma II.4: $\mathcal{C}_{\max }(I):=\left\{U^{\mathcal{A}}(\tilde{g}), \tilde{g} \in P S L(2, \mathbb{R})^{\sim}\right\}^{\prime} \cap \mathcal{B}(I)$ defines a Coset model associated with $\mathcal{A} \subset \mathcal{B}$. Every Coset model $\mathcal{C}$ associated with $\mathcal{A} \subset \mathcal{B}$ satisfies $\mathcal{C}(I) \subset \mathcal{C}_{\max }(I)$. The maximal Coset model $\mathcal{C}_{\max }$ associated to a subnet $\mathcal{A} \subset \mathcal{B}$ satisfies $\mathcal{C}_{\text {max }}(I)=\mathcal{A}^{\prime} \cap \mathcal{B}(I)$.

Proof: Obviously this definition yields a subtheory $\mathcal{C}_{\max } \subset \mathcal{B}$. Since the operators of a local algebra of $\mathcal{C}_{\text {max }}$ commute with the inner implementation of $\mathcal{A}$, we deduce from locality of $\mathcal{B}$ that $\mathcal{C}_{\max }$ is in fact a CosET model. 
Let $\mathcal{C}$ be any Coset model, $I, J$ proper intervals satisfying $I \subset J$ and $I^{\prime} \cup J=$ $\mathrm{S}^{1}$. By isotony of $\mathcal{C}$, locality and weak additivity (see below) for chiral subtheories we have: $\mathcal{C}(I) \subset\left(\mathcal{A}\left(I^{\prime}\right) \vee \mathcal{A}(J)\right)^{\prime}=\mathcal{A}^{\prime} \subset\left\{U^{\mathcal{A}}(\tilde{g}), \tilde{g} \in P S L(2, \mathbb{R})^{\sim}\right\}^{\prime}$.

The characterisation of $\mathcal{C}_{\max }$ as a subtheory which commutes with a representation of $P S L(2, \mathbb{R})^{\sim}$ is analogous to that of maximal chiral observables in a 1+1-dimensional conformal theory [Reh00]. It turns out that this analogy is quite complete; see section IV.2.

\subsection{Discussing the assumptions}

\section{Geometric modular action on chiral nets}

The vacuum, $\Omega$, is cyclic and separating for each local algebra $\mathcal{B}(I), I \Subset \mathrm{S}^{1}$, by locality and the REEH-SCHLIEDER theorem [RS61, Bor68] (cf [GF93, FJ96]), which means that the closure of $\mathcal{B}(I) \Omega$ coincides for every $I \Subset \mathrm{S}^{1}$ with the whole Hilbert space, $\mathscr{H}$, and for a local observable $B \Omega=0$ implies $B=0$.

Hence, it is possible to apply Tomita-Takesaki theory ${ }^{2}$, also called modular theory, which is of particular use for local quantum physics (cf [Bor00]). The fundamental structures of modular theory are contained in the Tomita-Takesaki theorem: Given a V.NEUMAnN algebra $\mathcal{M}$ with a cyclic and separating vector $\Omega$, there is a positive, invertible operator, $\Delta$, called modular operator, and an anti-unitary involution $J$, called modular conjugation, such that $J \mathcal{M} J=\mathcal{M}^{\prime}$ and $\sigma_{t}(\mathcal{M}):=\Delta^{i t} \mathcal{M} \Delta^{-i t}=\mathcal{M}, t \in \mathbb{R}$. The automorphism group $\sigma$ is called the modular group. The operators $J, \Delta$ form the modular data of the pair $(\mathcal{M}, \Omega)$ and they satisfy: $J \Omega=\Omega=\Delta \Omega, J=J^{*}=J^{-1}, \Delta^{i t}=J \Delta^{i t} J . J$ and $\Delta$ are given by the polar decomposition of Tomita's operator $S=J \Delta^{\frac{1}{2}}$, which is defined densely by: $S M \Omega=M^{*} \Omega, M \in \mathcal{M}$.

Every local algebra of $\mathcal{B}$ has its modular data because of the REEH-SCHLIEDER theorem, but modular theory becomes really useful for studies on quantum field theories, if one can make contact with the geometric net structure underlying the theory. In case one has such a geometrical interpretation of modular data, the theory is said to have the Bisognano-Wichmann property ${ }^{3}$. In general, such links are hard to establish, but conformally covariant theories are a remarkable exception [BGL93]. Taking $\mathcal{M}=\mathcal{B}\left(\mathrm{S}_{+}^{1}\right)$ yields in our setting: the modular group is directly related to the scale transformations according to $\Delta^{i t}=U(D(-2 \pi t))$, and the modular conjugation $J$ implements the reflection $x \mapsto-x, x \in \mathbb{R}$ [GF93, FJ96].

Guido, Longo And Wiesbrock have shown the following: A local net $\mathcal{B}$ on the chiral light-ray, which is covariant with respect to a representation $U$ of the translation-dilatation group 4 , extends to a conformal net, if and only

\footnotetext{
${ }^{2}$ See [BR87], [KR86], [SZ79], [Str81], [Tak70].

${ }^{3}$ Bisognano And Wichmann were the first to establish such a connection [BW75, BW76].

${ }^{4}$ This group is given as semi-direct product of the translations $T$ and the dilatations $D$ with
} 
if we have $U(D(-2 \pi t))=\Delta^{i t}$ for the modular operator of the algebra $\mathcal{B}\left(\mathrm{S}_{+}^{1}\right)$ [GLW98, theorem 1.4]. Thus one can not have dilatation-translation covariance and the BISOGNANO-WICHMANN property in the indicated form without having conformal covariance.

The local algebras $\mathcal{B}(I), I \Subset \mathrm{S}^{1}$, are continuous from the inside as well as from the outside, that is: $\mathcal{B}(I)$ coincides with the intersection of all local algebras assigned to proper intervals $J$ containing $\bar{I}$ and is generated by all its local subalgebras assigned to proper intervals $J$ with $\bar{J} \subset I$, respectively. Continuity from the inside implies weak additivity, ie $\mathcal{B}(I)$ is generated by the subalgebras $\mathcal{B}\left(J_{i}\right)$ for each covering $\bigcup_{i} J_{i}=I$ [FJ96]. The crucial continuity argument leading to these properties depends on scale invariance and stems from [LRT78, lemma II.2.2].

Uniqueness of the vacuum (up to scalar multiples) implies that the local algebras are factors, to be precise type $I I I_{1}$ factors [Dri75]. Type $I I I$ factors $\mathcal{M}$ have the specific property that any of their non-trivial projections $P<\mathbb{1}$ has infinite dimension and is equivalent in $\mathcal{M}$ to $\mathbb{1}$, ie there is an isometry $W \in \mathcal{M}$ satisfying $P=W W^{*}, \mathbb{1}=W^{*} W$. Using these properties, it is straightforward to show that type $I I I$ factors possess cyclic and separating vectors and that an algebraic isomorphism between type $I I I$ factors may always be implemented by a unitary operator (eg [Sch67]).

According to ConNes' classification of type $I I I$ factors [Con73], the $I I I_{1}$ factors are characterised by the following properties: the action of the modular group is outer, ie there is no one-parameter group of unitaries in the algebra itself which could implement the modular group, and the action of the modular group is ergodic, ie every operator left invariant by the modular group is a multiple of $\mathbb{1}$.

Ergodicity of the modular group may be deduced from the BisognANOWichmann property: If $B \in \mathcal{B}\left(\mathrm{S}_{+}^{1}\right)$ fulfills $\Delta^{i t} B \Delta^{-i t}=B$, then $B \Omega$ is in fact invariant with respect to the whole representation $U$ (lemma A.1). By the separating property of the vacuum, we get $B=U(g) B U(g)^{*}$ for all $g \in P S L(2, \mathbb{R})$. Covariance, locality and irreducibility then force $B$ to be a scalar multiple of $\mathbb{1}$. Factoriality is established quite easily and the outerness of the modular group follows from results contained in [Con73], see discussion in [Dri75].

In fact, uniqueness of the vacuum is equivalent to irreducibility of the net $\mathcal{B}\left(\bigvee_{I \Subset S^{1}} \mathcal{B}(I)\right.$ coincides with the algebra of all bounded operators on $\left.\mathscr{H}\right)$, factoriality of local algebras, and triviality of local algebras associated with points $\left(\bigcap_{I \Subset S^{1}, I \ni \zeta} \mathcal{B}(I)=\mathbb{C} \mathbb{1}\right)[$ GL96].

By covariance, the BISOGNANO-WICHMANN property of $\mathcal{B}$ means in particular: the vacuum representation of $\mathcal{B}$ satisfies Haag duality (on the circle), namely we have $\mathcal{B}(I)^{\prime}=\mathcal{B}\left(I^{\prime}\right), I \Subset \mathrm{S}^{1}$. If we simply talk of "HAAG duality" in the following, we will always mean "HAAG duality on $\mathrm{S}^{1}$ ". In physical terms, HAAG duality

the relation $D(t) T(a) D(-t)=T\left(e^{t} a\right)$. 
says that the local algebras can not be extended without violating locality. It is a very useful feature of a theory, if one wants to study its representations.

\section{Representations of chiral nets}

In $S^{1}$ the causal complement of each localisation region is again a localisation region. This is different from the situation for POINCARÉ covariant theories in $3+1$ dimensions or, indeed, for chiral nets on the light-ray: here the localisation regions are taken to be bounded which results in unbounded causal complements. On the chiral light-ray the causal complement of a bounded localisation region even consists of two disconnected components; the same is true in 1+1-dimensional spacetime. It is a general feature of conformal covering spaces that causal complements are themselves localisation regions [BGL93]. As a consequence of this, all locally normal representations turn out to be localisable in the sense of DoPLICHER, HAAG AND RoBERTS (DHR) [DHR69a].

A representation $\pi$ of a chiral conformal theory is a set of homomorphisms $\pi_{I}: \mathcal{B}(I) \rightarrow \pi_{I}(\mathcal{B}(I)), I \Subset \mathrm{S}^{1}$, into algebras of bounded operators on some HILBERT space $\mathscr{H}_{\pi}$, where the $\pi_{I}$ are required to fulfill the consistency relation $\pi_{I}\left\lceil\mathcal{B}(J)=\pi_{J}\right.$ for $J \subset I$. This family lifts uniquely to a representation $\pi$ of $\mathcal{B}_{\text {uni }}$, the universal $C^{*}$-algebra generated by all $\mathcal{B}(I), I \Subset \mathrm{S}^{1}$, and the $\pi_{I}$ are given in terms of the embeddings $\iota_{I}: \mathcal{B}(I) \hookrightarrow \mathcal{B}_{\text {uni }}$ by $\pi_{I}=\pi \circ \iota_{I}$ [Fre90, GL92] (cf eg [Sch97]).

Local normality of $\pi$ says that the local representations $\pi_{I}$ are normal (weak* continuous). The physical relevance of this property is discussed, for example, in [Haa92]. A representation $\rho$ is said to be localised in some $I_{0} \Subset \mathrm{S}^{1}$, if we have $\mathscr{H}_{\rho}=\mathscr{H}$ and $\rho \uparrow \mathcal{B}\left(I_{0}^{\prime}\right)=i d$. Any locally normal representation of a chiral conformal net is unitarily equivalent to a localised representation, ie localisable. This is due to the fact that the local algebras are type III factors.

Local normality is automatic if the representation lives on a separable HILBERT space (cf [GL96]). It follows as well, if a representation is covariant with positive energy and the global algebra $\pi(\mathcal{B}):=\bigvee_{I \Subset \mathrm{S}^{1}} \pi_{I}(\mathcal{B}(I))^{\prime \prime}$ possesses a cyclic vector. This is content of a theorem of BuchHolz, MACK AND Todorov [BMT88, theorem 1]; the original proof is very brief and we consider it worth while to make available a detailed proof in appendix A.4.

Locally normal representations of a local quantum theory are gathered in unitary equivalence classes, the superselection sectors, and the localisable representations form the class of DHR sectors. Conformal symmetry ensures that all sectors of a conformal theory are of DHR type. The fact that the set of localisation regions is not directed, ie that there are pairs of proper intervals $I_{1}, I_{2} \Subset \mathrm{S}^{1}$ to which there is no proper interval containing both of them, necessitates a generalisation of the standard treatment of superselection theory of DHR sectors [DHR69a, DHR69b, DHR71, DHR74]. Such a generalisation has been established by Fredenhagen, Rehren And Schroer (FRS) [FRS89, FRS92] 
(reviews eg [KMR90, Sch97]). Although the general structures of DHR theory carry over, there occur some striking differences. In particular, presence of non-trivial braid group statistics prohibits the application of the reconstruction method of Doplicher AND RoBERTs [DR90], which identifies in higher dimensions the theory of DHR sectors as a result of the action of a global gauge group on a field algebra and leaving fixed the observables.

\section{Haag duality on the light-ray}

In both the DHR theory and its generalisation by FRS, HAAG duality is fundamental. But while HAAG duality on $\mathrm{S}^{1}$ follows from the general assumptions, HAAG duality for the restricted net on the light-ray may be violated. A bounded interval $I$ on the light-ray $\mathbb{R}$, denoted $I \Subset \mathbb{R}$, has a causal complement $I^{\perp} \subset \mathbb{R}$ which, as a subset of the covering space $S^{1}$, consists of two disjoint, proper intervals $I_{ \pm} \Subset \mathrm{S}^{1}: I^{\perp}:=\mathrm{S}^{1} \backslash \bar{I} \cup\{\infty\}=I_{+} \cup \dot{\cup} I_{-}$. HAAG duality on the light-ray is valid, by definition, if we have: $\mathcal{B}(I)^{\prime}=\mathcal{B}\left(I_{+}\right) \vee \mathcal{B}\left(I_{-}\right)$. This identity does not hold in large classes of models [BSM90, Yng94].

It is not difficult to show that HAAG duality on the light-ray for chiral conformal theories is equivalent to strong additivity of local algebras (eg [GLW98, lemma 1.3]): a theory $\mathcal{B}$ is said to be strongly additive, if for every disjoint decomposition $I=I_{1} \dot{\cup}\{\zeta\} \dot{\cup} I_{2}, \zeta \in I \Subset \mathrm{S}^{1}$, we have $\mathcal{B}(I)=\mathcal{B}\left(I_{1}\right) \vee \mathcal{B}\left(I_{2}\right)$, ie the local algebra $\mathcal{B}(I)$ is, as a V.NEUMANN algebra, generated by its subalgebras $\mathcal{B}\left(I_{1}\right)$ and $\mathcal{B}\left(I_{2}\right)$. If this property holds for one such decomposition, then for all by covariance.

If a subnet $\mathcal{A} \subset \mathcal{B}$ is strongly additive, then the isotony problem is absent, ie the local relative commutants are automatically isotonuous. For a pair $I_{1,2}$ of proper intervals, $\overline{I_{1}} \subset I_{2}$, the latter possesses a disjoint decomposition $I_{2}=$ $I_{l} \dot{\cup}\left\{\zeta_{l}\right\} I_{1} \dot{\cup}\left\{\zeta_{r}\right\} \dot{\cup} I_{r}$, where $\zeta_{l, r}$ denote the boundary points of $I_{1}$ and $I_{l, r}$ are proper intervals. Locality implies under the assumption of strong additivity of $\mathcal{A}$ :

$$
\mathcal{C}_{I_{1}} \subset \mathcal{A}\left(I_{l}\right)^{\prime} \cap \mathcal{A}\left(I_{1}\right)^{\prime} \cap \mathcal{A}\left(I_{r}\right)^{\prime}=\mathcal{A}\left(I_{2}\right)^{\prime} .
$$

Examples of strongly additive theories are the stress-energy tensors with $c \leq 1$ [KL02, Xu03] and the chiral current algebras [BSM90, TL97].

It is always possible to construct from a non-strongly additive chiral conformal theory $\mathcal{B}$ a strongly additive one by taking the dual net $\mathcal{B}^{d}$ (cf [GLW98]). One first defines the local algebras of its restriction to the light-ray to be:

$$
\mathcal{B}^{d}(I):=\left(\mathcal{B}\left(I_{+}\right) \vee \mathcal{B}\left(I_{-}\right)\right)^{\prime}, I \Subset \mathbb{R} .
$$

As for its analogue in higher dimensions, the duality property for half-lines 5 , ie $\mathcal{B}\left(\mathbb{R}_{+}\right)^{\prime}=\mathcal{B}\left(\mathbb{R}_{-}\right)$, ensures locality for the dual net. The Bisognano-WiChmann

\footnotetext{
${ }^{5}$ Corresponding to duality for wedges in higher dimensions; this wedge duality usually is called essential duality. In our setting essential duality is identical to HAAG duality on $\mathrm{S}^{1}$.
} 
property of $\mathcal{B}$ yields conformal covariance of the dual net [GLW98, theorem 1.4]. By construction, both theories live on the same vacuum HiLBERT space.

The dual net $\mathcal{B}^{d}$ is an extension of $\mathcal{B}$ only upon restriction to the light-ray and not as a net on $\mathrm{S}^{1}$ and it has, in general, different physical properties. For example the superselection theory might be different, in particular due to the possible occurrence of soliton sectors [GLW98]. So, absence of strong additivity in a chiral conformal theory has to be taken seriously.

For the following another aspect is illuminating: conformal covariance is implemented on $\mathcal{B}^{d}$ by a representation $U^{d}$ of $P S L(2, \mathbb{R})$, which agrees with the implementation on $\mathcal{B}$, namely $U$, when restricted to the dilatation-translation subgroup; $U^{d}$ and $U$ are different as representations of $P S L(2, \mathbb{R})$, if $\mathcal{B}$ is not strongly additive. The following proposition is a simple variant of [GLW98, lemma 1.3]:

Proposition II.5: Let $\mathcal{B}$ be a chiral conformal net, covariant with respect to the representation $U$ of $P S L(2, \mathbb{R})$, and $\mathcal{B}^{d}$ its dual net, covariant with respect to the representation $U^{d}$. Then the following are equivalent:

i) $\mathcal{B}$ satisfies HAAG duality on the light-ray.

ii) $\mathcal{B}$ is strongly additive.

iii) $\mathcal{B}$ and $\mathcal{B}^{d}$ coincide.

iv) $U$ and $U^{d}$ coincide.

Proof: The equivalence i) $\Leftrightarrow$ ii) is established in [GLW98, lemma 1.3]. iii) is equivalent with ii) by the very definition of the dual net, (II.8), and HAAG duality.

The Bisognano-Wichmann property of chiral conformal theories establishes iii) $\Rightarrow$ iv), because the one-parameter subgroups of $\operatorname{PSL}(2, \mathbb{R})$ which leave the boundary points of some proper interval fixed coincide, when represented through $U$ and $U^{d}$, with the modular groups of the respective local algebra, and together these generate all of $P S L(2, \mathbb{R})$.

The opposite direction is implied by modular theory, too. Covariance allows us to deal with the situation for a single proper interval. For $I \Subset \mathbb{R}$ we have the inclusion $\mathcal{B}(I) \subset \mathcal{B}^{d}(I)$. By assumption iv) and the Bisognano-Wichmann property of $\mathcal{B}^{d}$ the subalgebra $\mathcal{B}(I)$ is globally invariant with respect to the action of the modular group of $\mathcal{B}^{d}(I)$. The remainder follows by a standard argument: first TAKESAKI's theorem [Tak72] on modular covariant subalgebras ensures existence of a normal, faithful conditional expectation from $\mathcal{B}^{d}(I)$ onto $\mathcal{B}(I)$, which leaves invariant the vacuum state. From here an argument of Jones [Jon83] establishes $\mathcal{B}(I)$ as the subalgebra of operators in $\mathcal{B}^{d}(I)$ which commute with the projection onto the closure of $\mathcal{B}(I) \Omega$, which is $\mathbb{1}$ by the REEH-SCHLIEDER theorem for $\mathcal{B}$. 


\section{Modular covariance of chiral subnets}

The assumption of conformal covariance, definition II.2,iii), introduces rich and powerful structures into the investigations of a chiral subnet $\mathcal{A} \subset \mathcal{B}$. Basically, this assumption requires the restricted nets $\mathcal{A}$ and $\mathcal{B}$ to have simultaneous conformal extensions preserving the inclusions for the extended nets on $\mathbf{S}^{1}$. This excludes interesting inclusions on the light-ray. One example is given by the relation between the strongly additive dual net $\mathcal{B}^{d}$ and its basis $\mathcal{B}$ (proposition II.5): the restriction of $\mathcal{B}$ to the light-ray is contained in the corresponding restriction of $\mathcal{B}^{d}$, both possess conformal extensions, but this extension can not be simultaneous, if $\mathcal{B} \neq \mathcal{B}^{d}$. The conformally covariant derivatives of the $U(1)$ current form a particular class of examples of this structure [GLW98, corollary 2.11].

Conformal covariance of the subnet $\mathcal{A} \subset \mathcal{B}$ implies two important facts: first, the chiral subnet possesses the REEH-SCHLIEDER property, ie the cyclic subspace generated by the global algebra $\mathcal{A}$ from the vacuum coincides with the cyclic subspace of any local algebra $\mathcal{A}(I), I \Subset \mathrm{S}^{1}$. This is a direct consequence of the REEH-SChlieder theorem as proved by Borchers [Bor68]. We write $e_{\mathcal{A}}$ for the cyclic projection of $\mathcal{A}$, ie we have: $e_{\mathcal{A}} \mathscr{H}=\overline{\mathcal{A}(I) \Omega}$.

While the REEH-SCHLIEDER property holds for dilatation-translation covariant subnets on the light-ray as well, in conformally covariant subnets each local inclusion $\mathcal{A}(I) \subset \mathcal{B}(I), I \Subset \mathrm{S}^{1}$, is globally left invariant by the modular group of $\mathcal{B}(I)$. Such modular covariant subalgebras have features which will be crucial in the following (cf section V、1): The cyclic projection $e_{\mathcal{A}}$ characterises completely the subalgebra $\mathcal{A}(I)$ in $\mathcal{B}(I)$, namely we have by TAKESAKI's theorem [Tak72] and an argument of JONES [Jon83]:

$$
\mathcal{A}(I)=\left\{e_{\mathcal{A}}\right\}^{\prime} \cap \mathcal{B}(I), I \Subset \mathrm{S}^{1} .
$$

In particular there are no modular covariant subalgebras of $\mathcal{B}(I)$ which generate a dense subspace from the vacuum other than $\mathcal{B}(I)$ itself.

The mapping $\mathcal{A}(I) \rightarrow \mathcal{A}(I) e_{\mathcal{A}}$ given by $A \mapsto A e_{\mathcal{A}}$ is known to define an isomorphism of V.NEUMANN algebras (because of the separating property of the vacuum, eg $[$ Bor97a] $)$. It is easy to see that the net $\mathcal{A} e_{\mathcal{A}}$ defines a chiral conformal theory in its vacuum representation, and it is readily checked that the inverse isomorphisms $\pi_{I}: \mathcal{A}(I) e_{\mathcal{A}} \rightarrow \mathcal{A}(I) \subset \mathcal{B}(I)$ define a locally normal $]^{6}$ representation of $\mathcal{A} e_{\mathcal{A}}$ due to equation II.9. Because of these local isomorphisms we will speak occasionally of "representations of $\mathcal{A}$ ", although these actually are representations of $\mathcal{A} e_{\mathcal{A}}$. We will never speak of representations of the global algebra $\mathcal{A}$ and so no confusion should arise.

\footnotetext{
${ }^{6}$ Algebraic isomorphisms of V.NEUMANN algebras are automatically ultra-weakly and ultrastrongly continuous [Dix81, I.4.3. corollary 1]. Since the local algebras are type $I I I$ factors, the isomorphism may be implemented by a unitary operator from $\mathscr{H}$ onto $e_{\mathcal{A}} \mathscr{H}$ [Sch67, II.4.6. theorem].
} 
TAKESAKI's theorem [Tak72] says the following as well: the vacuum state, $\omega()=.\langle\Omega, \Omega\rangle$, is a product state on every v.NEUMANN algebra $\mathcal{A}(I) \vee \mathcal{C}_{I}$ :

$$
\omega(A C)=\omega(A) \omega(C), A \in \mathcal{A}(I), C \in \mathcal{C}_{I}, I \Subset S^{1} .
$$

Because of this product state, the mapping $\mathcal{A}(I) \vee \mathcal{C}_{I} \ni \sum_{i} a_{i} c_{i} \mapsto \sum_{i} a_{i} \otimes c_{i} \in$ $\mathcal{A}(I) \otimes \mathcal{C}_{I}$ extends to an isomorphism from $\mathcal{A}(I) \vee \mathcal{C}_{I}$ onto $\mathcal{A}(I) \otimes \mathcal{C}_{I}$.

For Coset pairs $\mathcal{A} \subset \mathcal{C} \subset \mathcal{B}$ the product state property of the vacuum has the following consequence:

Proposition II.6: Let $\mathcal{A} \subset \mathcal{C} \subset \mathcal{B}$ be a Coset pair. The chiral conformal theories $\mathcal{A} \subset \mathcal{C} e_{\mathcal{A} \odot \mathcal{C}}$ and $\mathcal{A} e_{\mathcal{A}} \otimes \mathcal{C} e_{\mathcal{C}}$ are unitarily equivalent.

Proof: Straightforward verification shows $\mathcal{A} e_{\mathcal{A}} \otimes \mathcal{C} e_{\mathcal{C}}$ to be a chiral conformal theory with the obvious definitions: its vacuum is given by $\Omega \otimes \Omega$, the representation implementing covariance is $\left(U e_{\mathcal{A}} \otimes U e_{\mathcal{C}}\right)($.$) , its representation space is$ $e_{\mathcal{A}} \mathscr{H} \otimes e_{\mathcal{C}} \mathscr{H}$. The factoriality of the local algebras proves that $\Omega \otimes \Omega$ is (up to scalar multiples) the unique vacuum [GL96, proposition 1.2], [Tak79, IV.5., corollary 5.11].

$\Omega$ is separating for $\bigcup_{I \in \mathbb{R}} \mathcal{A} \subset \mathcal{C}(I) e_{\mathcal{A} \subset \mathcal{C}}$, the union of all local algebras assigned to bounded intervals in $\mathbb{R}$. Thus, we are allowed to define a linear operator $W$ densely by:

$$
W A C \Omega:=A \Omega \otimes C \Omega, A \in \mathcal{A}(I), C \in \mathcal{C}(I), I \Subset \mathbb{R} .
$$

On the algebra $\bigcup_{I \Subset \mathbb{R}} \mathcal{A} \subset \mathcal{C}(I) e_{\mathcal{A} \subseteq \mathcal{C}}$ the vacuum is a product state (a corollary to TAKESAKI's theorem [Tak72]). Hence, $W$ is bounded and extends by continuity to an isometry, as one may readily verify.

We may check from the definition, that $W$ is an intertwiner:

$$
W A_{1} C_{1} A_{2} C_{2} \Omega=\left(A_{1} \otimes C_{1}\right) W A_{2} C_{2} \Omega, A_{1,2} \in \mathcal{A}(I), C_{1,2} \in \mathcal{C}(I), I \Subset \mathbb{R} .
$$

Thus, $W W^{*}$ and $W^{*} W$ commute with the respective restricted nets on $\mathbb{R}$, but these are irreducible, as follows from irreducibility of the respective conformal nets on $\mathrm{S}^{1}$ using weak additivity, HAAG duality and the factor property of the algebras assigned to $\mathrm{S}_{+}^{1}$. Thereby, $W$ is a unitary operator.

$A d_{W}$ induces a unitary equivalence of the respective local algebras associated with every $I \Subset \mathbb{R}$ by its definition (II.11) and the separating property of the vacuum. Furthermore, $W$ is readily shown to be covariant. If we denote the covariance automorphisms of $\mathcal{A} e_{\mathcal{A}} \otimes \mathcal{C} e_{\mathcal{C}}$ by $\alpha^{\otimes}$, we have for $g I \Subset \mathbb{R}, I \Subset \mathbb{R}$ : $\alpha_{g}^{\otimes} A d_{W}\left\lceil\mathcal{A}\left(\mathcal{C}(I)=A d_{W} \alpha_{g} \uparrow \mathcal{A}(\mathcal{C}(I)\right.\right.$.

Using the REEH-SCHLIEDER property of the local algebras, one may reconstruct the representations $\left(U e_{\mathcal{A}} \otimes U e_{\mathcal{C}}\right)($.$) and U(.) e_{\mathcal{A} \odot \mathcal{C}}$ from the action of the automorphisms. This, in turn, proves that $W$ intertwines the representations $U(.) e_{\mathcal{A} \odot \mathcal{C}}$ and $\left(U e_{\mathcal{A}} \otimes U e_{\mathcal{C}}\right)($.$) . Finally, we reconstruct the conformal models$ 
from their restrictions by applying conformal covariance.

Because of this proposition we will denote the vacuum representation of a Coset pair $\mathcal{A} \subset \mathcal{C} \subset \mathcal{B}$ by $\mathcal{A} \otimes \mathcal{C}$; hence, we regard the inclusion $\mathcal{A} \subset \mathcal{C} \subset \mathcal{B}$ as a locally normal representation of $\mathcal{A} \otimes \mathcal{C}$.

Even in the case of finite index (see below) it is not clear, whether the representation induced by the inclusion $\mathcal{A} \subset \mathcal{C} \subset \mathcal{B}$ has a spatial decomposition into tensor products of representations of $\mathcal{A} e_{\mathcal{A}}$ and $\mathcal{C} e_{\mathcal{C}}$, respectively. The examples we study in chapter $\mathrm{V}$ have such a spatial decomposition and there are conditions which ensure this structure [KLM01, lemma 27].

\section{Split property for chiral subnets}

One of the properties which are expected to hold true in every physically decent local quantum theory is the split property. In physical terms, the split property ensures that observables which are sufficiently spacelike separated do not only commute, but actually become statistically independent $t^{7}$. There are equivalent formulations of this property, but the usual definition (adapted to our context; cf [GF93, definition 2.11]) is: a chiral net $\mathcal{B}$ has the split property, if for any pair $I_{1,2}$ of proper intervals satisfying $\overline{I_{1}} \subset I_{2}$ there is a type $I$ factor $\mathcal{M}$ interpolating between $\mathcal{B}\left(I_{1}\right)$ and $\mathcal{B}\left(I_{2}\right)$, ie:

$$
\mathcal{B}\left(I_{1}\right) \subset \mathcal{M} \subset \mathcal{B}\left(I_{2}\right), \overline{I_{1}} \subset I_{2} \Subset \mathrm{S}^{1} .
$$

The split property is implied by the condition of nuclearity which, loosely speaking, says that finite volumes in classical phase space should correspond to almost finite-dimensional parts in state space of quantum physics. Nuclearity ensures, in particular, decent thermodynamic properties of a theory. A technical formulation was given and established for the free scalar Hermitian field by BuchHolz And Wichmann [BW86]. For a general summary on the notions of split property and nuclearity see eg [Haa92, V.5].

The split property has far reaching consequences. First to name is the (almost) complete determination of the type of local algebras. BuchHolz, D'AnToni AND FREDENHAGEN [BDF87] proved the local algebras to be the tensor product of their centre and the hyperfinite type $I I I_{1}$ factor; the latter is unique up isomorphism [Haa87]. Another important consequence is a general quantum version of NoETHER's theorem [BDL86, DDFL87].

In [BDF87] the formulation is given for nets in 3+1-dimensional MinkowskI spacetime, but it was translated for chiral nets by GABBIANI AND FröHLICH [GF93, lemma 2.12, theorem 2.13]. In typical chiral models $e^{-\beta L_{0}}, \beta>0$, is trace-class and nuclearity follows from the asymptotic properties of its trace in the limit $\beta \searrow 0$ (see section VI.1); a very general discussion on this aspect of

\footnotetext{
${ }^{7}$ For a discussion of different notions of statistical independence see eg [Sum90].
} 
nuclearity for chiral conformal theories is contained in [DLR01, theorem 3.2., lemma 3.3].

From the very formulation of the nuclearity condition (see equations (VI.6), (VI.7)), it is obvious, that any chiral subnet $\mathcal{A} \subset \mathcal{B}$ inherits nuclearity and hence the split property. It is remarkable that the split property can be shown directly to be passed on from $\mathcal{B}$ to the subnet $\mathcal{A}$ by standard methods [CC01, proof of proposition 2.3]. As every Coset model $\mathcal{C}$ is a chiral subnet, the following proposition applies to them in particular:

Proposition II.7: Let $\mathcal{A} \subset \mathcal{B}$ be a chiral subnet. If $\mathcal{B}$ has the split property (is nuclear), then $\mathcal{A}$ has the split property (is nuclear).

Proof: There is nothing to prove with respect to nuclearity. We discuss the split property for the restriction of $\mathcal{A}$ to its vacuum subrepresentation $\mathcal{A} e_{\mathcal{A}}$. According to arguments in [Buc74] we only need to prove a faithful normal product state $\phi$ to exist on $\mathcal{A}\left(I_{1}\right) e_{\mathcal{A}} \vee \mathcal{A}\left(I_{2}^{\prime}\right) e_{\mathcal{A}}$, if $\overline{I_{1}} \subset I_{2}$.

From $\overline{I_{1}} \subset I_{2}$ we conclude that there is $I_{3} \Subset \mathrm{S}^{1}$ satisfying $I_{1} \cup I_{2}^{\prime} \subset I_{3}$, and because of modular covariance $\eta: \mathcal{A}\left(I_{3}\right) \rightarrow \mathcal{A}\left(I_{3}\right) e_{\mathcal{A}}$, given by $A \mapsto A e_{\mathcal{A}}$, defines an isomorphism of V.NEUMANN algebras. Upon restriction, $\eta$ becomes an isomorphism from $\mathcal{A}\left(I_{1}\right) \vee \mathcal{A}\left(I_{2}^{\prime}\right)$ onto $\mathcal{A}\left(I_{1}\right) e_{\mathcal{A}} \vee \mathcal{A}\left(I_{2}^{\prime}\right) e_{\mathcal{A}}$. Hence, we get the desired state $\phi$ by taking a normal, faithful product state $\psi$ on $\mathcal{B}\left(I_{1}\right) \vee \mathcal{B}\left(I_{2}^{\prime}\right)$ (which exists since $\mathcal{B}$ is split) and setting: $\phi:=\psi \circ \eta^{-1}$.

\section{On the isotony problem of chiral subnets}

A chiral subnet $\mathcal{A} \subset \mathcal{B}$ is called cofinite (cf [Xu00a]), if the inclusion $\mathcal{A} \subset \mathcal{C}_{\text {max }} \subset \mathcal{B}$ is of finite index, which is equivalent to the requirement that the DHR endomorphism of $\mathcal{A} \otimes \mathcal{C}_{\max }$ which induces the representation $\mathcal{A} \subset \mathcal{C}_{\max } \subset \mathcal{B}$ has finite statistics $^{8}$. In general it is hard to prove that a subnet $\mathcal{A} \subset \mathcal{B}$ is cofinite. However, $\mathrm{XU}[\mathrm{Xu} 00 \mathrm{a}]$ was able to prove a large class of current subalgebras being cofinite. Cofiniteness of $\mathcal{A} \subset \mathcal{B}$ has remarkable consequences, like: $\mathcal{B}$ is strongly additive, if and only if both $\mathcal{A}$ and $\mathcal{C}_{\max }$ are [Lon01]. The current subalgebras considered by XU are strongly additive [TL97, corollary IV.1.3.3.].

Yet, finiteness of index is not a general assumption. For example, REHREN studied the chiral subnet induced by the inclusion of the stress-energy tensor of central charge $c=1$ in the current algebra $\mathcal{L} S U(2)_{1}$ and found that this inclusion does not have a finite index [Reh94a]. Furthermore, CARPI [Car02] has shown that sectors of the theory generated by this stress-energy tensor typically do not have finite statistical dimension.

So, even if both $\mathcal{A}$ and $\mathcal{B}$ are strongly additive, it is unclear, in general,

\footnotetext{
${ }^{8}$ More details on inclusions with finite index in chapter $\mathrm{V}$.
} 
whether $\mathcal{C}_{\max }$ or any other non-trivial COSET model is strongly additive $e^{9}$. If we now look at the subnet $\mathcal{C}_{\max } \subset \mathcal{B}$ and consider its CosET models, we arrive at the isotony problem for this inclusion. In case $\mathcal{C}_{\max }$ is strongly additive as well, it is obvious that $\mathcal{C}_{\max } \subset \mathcal{B}$ and its maximal COSET model are locally their mutual relative commutants. Inclusions of this type are of particular interest; REHREN called them normal pairs of subtheories [Reh00]. Hence, we have reasons to take the isotony problem serious, even if we start with an inclusion of strongly additive theories.

We would like to have a simple and applicable characterisation of local observables in $\mathcal{B}$ which belong to a CosET model associated with a subtheory $\mathcal{A}$, and we would like this characterisation to involve only local data following the conviction that every observation is of finite extension and of finite duration. Of course, it is possible to make this decision simply by taking all operators from $\mathcal{C}_{I}$ and discarding all operators which do not commute with all operators belonging to an algebra $\mathcal{A}(J), J$ slightly enlarged. But such a method will in most cases not prove useful when looking at a particular model and, furthermore, chiral conformal quantum field theories usually behave well when taking the limit $J \rightarrow I$. So, we are led to the conjecture that equality $\mathcal{C}_{I}=\mathcal{C}_{\max }(I)$ should hold in very general circumstances.

As it stands at the moment, the maximal COSET model is determined by global data, the inner-implementing representation $U^{\mathcal{A}}$, and establishing the equality $\mathcal{C}_{I}=\mathcal{C}_{\text {max }}(I)$ would prove that all CosET models are of a local nature, their local operators being singled out by a simple algebraic relation only involving local data associated with the very same localisation region, namely the commutativity with the operators of $\mathcal{A}(I)$.

For dealing with the isotony problem in our context 10 , we look at the action of $A d_{U^{\mathcal{A}}}$ on the local observables of $\mathcal{B}$. Because the construction of $U^{\mathcal{A}}$ does not refer to the local structure of $\mathcal{A}$ at all (see section 2), we need some more information on the way this representation is generated by local observables.

In chiral conformal field theory it is natural to assume that $U^{\mathcal{A}}$ is generated by integrals of a stress-energy tensor affiliated with $\mathcal{A}$. This assumption does not imply strong additivity [BSM90] and concerning the models known today seems more general, because all strongly additive models do contain a stress-energy tensor. Because of the special features of stress-energy tensors in chiral (and 1+1-dimensional) conformal field theory, mainly due to the LüsCHER-MACK

\footnotetext{
${ }^{9}$ Not even for current subalgebras the situation has been clarified. Relying on results of WASSERMAnN [Was98, theorem E], XU claimed strong additivity to hold for the maximal CoseT model of current subalgebras $\mathcal{A} \subset \mathcal{B}, \mathcal{B}$ a current algebra associated with $S U(n)$ [Xu00a], but the method of proof for [Was98, theorem E] is not valid, actually, and thus the claim was withdrawn [Xu01].

${ }^{10}$ Apparently, CARPI AND CONTI encountered the same problem while generalising their analysis [CC01] to general field algebras and solved it by methods quite different from the ones applied here $[\mathrm{CC}]$.
} 
theorem [FST89, Mac88, LM76], this assumption admits a successful discussion of the isotony problem, see chapter IV. But the presence of a stress-energy tensor does not trivialise the problem at all. In fact, one is led to pinpoint the problem using general arguments, before the stress-energy tensor actually is needed to prove two crucial lemmas. Our discussion should, therefore, serve well as a setup for further generalisations.

Even for current subalgebras, which always contain a stress-energy tensor by the Sugawara construction, the action of the stress-energy tensor $\Theta^{\mathcal{A}}$ of a current subalgebra on general currents in the larger current algebra $\mathcal{B}$ has not been studied as such, yet. Only in connection with the classification of conformal inclusions, ie the case that the stress-energy tensor $\Theta^{\mathcal{B}}$ coincides with that of $\mathcal{A}$ [SW86, AGO87, BB87], this action has been object of research, however, by quite indirect methods. The new perspective of analysing the action of $U^{\mathcal{A}}$ on $\mathcal{B}$ (in this context: of $\Theta^{\mathcal{A}}$ on $\mathcal{B}$ ) has led to a simple characterisation of conformal inclusions by methods familiar in (axiomatic) quantum field theory, see section III.4. There is a natural notion of conformal inclusion of chiral nets in our broader approach (definition II.21).

Our analysis directly applies to the maximal CosET models of current subalgebras, because these contain the CosET stress-energy tensor $\Theta^{\mathcal{B}}-\Theta^{\mathcal{A}}$. This way we extend the finding on normal pairs for cofinite current subalgebras to all inclusions $\mathcal{A} \subset \mathcal{B}$ where both $\mathcal{B}$ and $\mathcal{A}$ contain a stress-energy tensor, independent of strong additivity or the index of the inclusion $\mathcal{A} \subseteq \mathcal{C}_{\max } \subset \mathcal{B}$.

We will give a summary on current subalgebras in chapter III and show them to have all the features which we assume to hold for our analysis. The isotony problem will be solved step by step in chapter IV. But, first we have to show that our notion of maximal CosET model applies to all chiral subnets by proving the inner-implementing representation associated with them to exist and to have properties required later on.

\section{Conformal transformations as observables}

Spacetime symmetries are of paramount importance to relativistic quantum field theory. Intuitively we expect such coordinate transformations to be connected to observables. Time translations, for example, should be observable due to their connection with the energy operator. If we have a stress-energy tensor in the theory, as it is often the case in models, the energy operator itself is given as an integral of this local quantum field. Yet, the implementation of covariance may be given in abstract terms or may stem from a larger theory into which the theory of interest is embedded, and it is not always manifest how covariance may be implemented by observables of the subtheory.

More specifically, as a fact of life any observation is of finite extension in space and time and thus we regard the local observables as the constituting objects in 
quantum field theory. For this reason we shall work with the V.NEUMANN algebra $\mathcal{A}$ which is generated by all local observables. Thereby our setting includes quantum field theories which are not necessarily described completely by covariant quantum fields, and which might not possess a stress-energy tensor. In fact, the main result of this section (theorem II.14) is an abstract statement about V.NEUMANN algebras, without reference to the local structure of a quantum field theory.

We consider representations of such theories which admit a unitary implementation of covariance, $V$, and the task thus amounts to a search for observable, unitary, implementing operators. Quite obviously these operators can not be local observables, since locality implies that adjoint action of these operators is trivial on algebras which are associated with causally disconnected regions. On the other hand we believe any observation has to be local in nature and we conclude: spacetime transformations should be non-local limits of local observables, ie genuine global observables.

The problem of identifying spacetime symmetry transformations as global observables is of interest only, if the given representation is reducible. In irreducible representations, such as the vacuum representation, any bounded operator can be represented as a weak limit of local operators. The representations induced by non-trivial chiral subnets $\mathcal{A} \subset \mathcal{B}$ are manifestly reducible, as we have for the cyclic projection: $e_{\mathcal{A}} \neq \mathbb{1}, 0$.

To our knowledge this problem so far has been dealt with only in the case of abelian groups of translations satisfying the spectrum condition (positivity of energy). Borchers [Bor66] has solved this problem relying almost entirely on the spectrum condition and using a deep result on the innerness of norm-continuous connected automorphism groups of V.NEUMANN algebras [KR67, corollary 8]. His result is the key building block in our construction.

In the abelian case there are many inner-implementing representations with different spectral properties. It was a challenging task to ensure existence of an inner-implementing representation satisfying the spectrum condition. ARveson Arv74] gave a proof for a one-parameter group, Borchers and BuchHOLZ [Bor84, BB85] succeeded in solving this problem in general; see [Bor87] for a summary.

The situation for an inner-implementing representation of $\operatorname{PSL}(2, \mathbb{R}) \sim$ is different. Because $P S L(2, \mathbb{R})^{\sim}$ is identical with its commutator subgroup, the result of our construction is unique and validity of the spectrum condition follows. We show as well that $V$-invariant vectors are left invariant by the action of the inner-implementing representation $V^{\mathcal{A}}$. Another result is the proof of complete reducibility of $V^{\mathcal{A}}$ under weak assumptions on the original representation $V$.

In the course of our argument we will construct an inner-implementing representation $V^{\mathcal{A}^{\prime}}$ for the commutant of the V.Neumann algebra $\mathcal{A}$ as well. We have the following relation: $V(\tilde{g})=V^{\mathcal{A}}(\tilde{g}) V^{\mathcal{A}^{\prime}}(\tilde{g}), \quad \forall \tilde{g} \in P S L(2, \mathbb{R})^{\sim}$. This equation reminds of the COSET construction [GKO86] involving stress-energy tensors 
of chiral current algebras, which are given by the SUGAWARA construction. It is not difficult to show that our result agrees with the outcome of integrating the respective stress-energy tensors (see chapter III).

Although the relation to CoseT constructions as considered by GODDARD, KENT AND OLIVE [GKO86] motivates the enterprise undertaken here, its result is independent of the existence of a stress-energy tensor. We have made use of this, already, and connected it to a generalised notion of COSET construction (lemma II.4). In subsection 2.2 we will give some more details on this and other applications to chiral subnets.

Although there are special features of an inner-implementing representation connected to a stress-energy tensor, the result of our construction serves well as a substitute for the SUGAWARA stress-energy tensor in many respects. On the other hand we believe our construction to be somewhat special to (chiral) conformal field theories as we argue in the discussion concluding this section, and we know that the deeper part of it is due to Borchers. Summing up these thoughts we consider the term Borchers-Sugawara construction appropriate.

Most of this section has been published already [Kös02], but there are some differences which we have indicated in the text. Moreover, we have included some additional material, in particular proposition II.20.

\subsection{BORCHERS-SUGAWARA construction}

In the following $\mathscr{H}$ stands for a separable HiLBERT space and $V$ is a unitary, strongly continuous representation of $P S L(2, \mathbb{R})^{\sim}$ on $\mathscr{H}$. If not stated otherwise, $\mathcal{A}$ stands for a v.NeUmAnN algebra of operators on $\mathscr{H}, \mathcal{A}^{\prime}$ for its commutant and $\alpha, \alpha^{\prime}$ for automorphic actions of $\operatorname{PSL}(2, \mathbb{R})^{\sim}$ on $\mathcal{A}, \mathcal{A}^{\prime}$ respectively. We note that any spatial automorphism of $\mathcal{A}$, given by the adjoint action of a unitary operator, induces a spatial automorphism of $\mathcal{A}^{\prime}$ as well.

We first prove a lemma on the spectrum condition. The result is well known and our proof is not new, presumably; its second part is adapted from [Mac77].

Proposition II.8: If any one of the operators $L_{0}, P,-K$ has positive spectrum, then all three of them. In this case we say that $V$ satisfies the spectrum condition.

Proof: Assume $L_{0}$ is positive. Take any vector $\phi$ analytic for the representation $V(\mathrm{cf}$ eg [BR77]). We have:

$$
0 \leqslant 2\left\langle\phi, V(\widetilde{D}(\tau)) L_{0} V(\widetilde{D}(-\tau)) \phi\right\rangle=e^{\tau}\langle\phi, P \phi\rangle+e^{-\tau}\langle\phi,-K \phi\rangle .
$$

Multiplying by $e^{ \pm \tau}$ and taking the appropriate limits $\tau \rightarrow \mp \infty$ we deduce $\omega_{\phi}(P) \geq 0$ and $\omega_{\phi}(-K) \geq 0$. Since the analytic vectors for the representation $V$ form a core for all generators we may apply criterion 5.6.21 of [KR83].

Now assume $P$ or $-K$ is positive. Special conformal transformations and translations are conjugate in $P S L(2, \mathbb{R}): S(-n)=R(\pi) T(n) R(-\pi)$. Defining 
$g_{t}:=S(n) R(t) T(n) R(-t)$ this identity becomes: $\lim _{t / \pi} g_{t}=i d$. Now we see that the corresponding relation holds true in $\operatorname{PSL}(2, \mathbb{R})^{\sim}$, since we know it for $\operatorname{PSL}(2, \mathbb{R})$, the relation is continuous in $n$ and the covering projection is continuous as well. Because conjugation by a unitary operator does not change the spectrum, positivity of $P$ follows from positivity of $-K$ and vice versa. Positivity of $L_{0}$ follows from equation (II.4) by criterion 5.6.21 of [KR83] applied as before.

Alternatively, one may prove proposition II.8 by decomposing any unitary representation satisfying the spectrum condition into a direct integral of irreducible representations (eg [BR77, chapter 5, §6, theorem 3]). The latter are known explicitly (eg [Gri93]) and the ones fulfilling the spectrum condition on $L_{0}$ have positive spectrum for the translations as well. This procedure is followed in the literature (eg [GL96, PS86, GF93]).

Proposition II.9: Assume $A d_{V}$ induces an automorphism group $\alpha$ on $\mathcal{A}$. If there exists a representation $V^{\mathcal{A}}$ of $\operatorname{PSL}(2, \mathbb{R})^{\sim}$ by unitary operators in $\mathcal{A}$ implementing $\alpha$ by its adjoint action on $\mathcal{A}$, then this representation is unique.

Proof: Assume there are two such representations, $V_{1}^{\mathcal{A}}$ and $V_{2}^{\mathcal{A}}$. Then the operators $V_{1}^{\mathcal{A}}(\tilde{g}) V_{2}^{\mathcal{A}}(\tilde{g})^{*}, \tilde{g} \in P S L(2, \mathbb{R})^{\sim}$, implement the trivial automorphism. For this reason these operators belong to the centre of $\mathcal{A}$. Using this fact it is straightforward to show that the operators $V_{1}^{\mathcal{A}}(\tilde{g}) V_{2}^{\mathcal{A}}(\tilde{g})^{*}$ form a representation of $P S L(2, \mathbb{R})^{\sim}$. This representation is abelian and its kernel contains all elements of the form $\tilde{g}_{1} \tilde{g}_{2} \tilde{g}_{1}^{-1} \tilde{g}_{2}^{-1}$.

Now these elements generate the whole of $\operatorname{PSL}(2, \mathbb{R})^{\sim}$ since it is a perfect group, ie it coincides with its commutator subgroup. One can reduce this statement on the LIE group to the structure of the corresponding LIE algebra by standard arguments (eg [HN91], lemmas III.3.19, III.3.20, definition I.5.1 and remarks nearby this definition). So, $P S L(2, \mathbb{R})^{\sim}$ is perfect since it has a simple LIE algebra and is connected. Thereby $V_{1}^{\mathcal{A}}(\tilde{g}) V_{2}^{\mathcal{A}}(\tilde{g})^{*}=\mathbb{1} \forall \tilde{g} \in P S L(2, \mathbb{R})^{\sim}$.

We call a representation $V^{\mathcal{A}}$ in the sense of the proposition above an innerimplementing representation (corresponding to the pair $(V, \mathcal{A})$ ). We immediately have:

Proposition II.10: Assume the unique inner-implementing representation $V^{\mathcal{A}}$ to exist. Then $V^{\mathcal{A}^{\prime}} \equiv V\left(V^{\mathcal{A}}\right)^{*}$ is the unique inner-implementing representation corresponding to $\left(V, \mathcal{A}^{\prime}\right)$. If $V^{\mathcal{A}}$ is strongly continuous, then so is $V^{\mathcal{A}^{\prime}}$.

Proof: First we prove innerness of the operators $V(\tilde{g}) V^{\mathcal{A}}(\tilde{g})^{*}$ by recognising that their adjoint action on $\mathcal{A}$ implements the trivial automorphism. Making use of this it is straightforward to show that these operators do in fact define a representation. The implementation property and unitarity are trivial. Uniqueness 
follows from proposition II.9 directly. Continuity is fulfilled, since we are multiplying continuous functions.

We now come to the derivation of the main result of this section (theorem II.14). It depends on the following statement:

Lemma II.11: Let $V$ satisfy the spectrum condition and let $A d_{V}$ induce an automorphism group $\alpha$ of $\mathcal{A}$. Then there are strongly continuous, unitary, innerimplementing representations $\widetilde{T}^{\mathcal{A}}, \widetilde{S}^{\mathcal{A}}, \widetilde{R}^{\mathcal{A}}$ for the restrictions of $\alpha$ to the oneparameter subgroups of translations, special conformal transformations and rotations, respectively.

Proof: This is an application of Borchers' theorem [Bor66] and proposition II.8.

At this point we stress that it is not clear at all whether these restricted innerimplementing groups form a representation of $\operatorname{PSL}(2, \mathbb{R})^{\sim}$. We will show that the inner-implementing representation may be constructed from any given pair $\widetilde{T}^{\mathcal{A}}$, $\widetilde{S}^{\mathcal{A}}$. The fact that there are sufficiently many subgroups satisfying the spectrum condition to generate the whole group seems to be special.

According to the IWASAwA decomposition of $P S L(2, \mathbb{R})$ [GF93] (appendix I) we can write every $g \in P S L(2, \mathbb{R})$ in the form $g=T\left(p_{g}\right) D\left(\tau_{g}\right) R\left(t_{g}\right)$. Each term in this decomposition depends continuously on $g$. By a short consideration on the covering projection from $P S L(2, \mathbb{R})^{\sim}$ to $P S L(2, \mathbb{R})$ we readily see that the same decomposition works for $P S L(2, \mathbb{R})^{\sim}$ as every element $\tilde{g}$ is of the form $\tilde{R}(2 \pi)^{m} g$, $m \in \mathbb{Z}$ and $g$ from the first sheet of the covering, which we may identify with $\operatorname{PSL}(2, \mathbb{R})$.

Again in $P S L(2, \mathbb{R})$ we may check that every dilatation and every rotation may be written as follows:

$$
\begin{aligned}
D(\tau) & =S\left(-\left(e^{\frac{\tau}{2}}-1\right) e^{-\frac{\tau}{2}}\right) T(1) S\left(e^{\frac{\tau}{2}}-1\right) T\left(-e^{-\frac{\tau}{2}}\right) \\
R(2 t) & =S\left((-1+\cos t)(\sin t)^{-1}\right) T(\sin t) S\left((-1+\cos t)(\sin t)^{-1}\right) .
\end{aligned}
$$

By looking at the curves in $P S L(2, \mathbb{R})^{\sim}$ defined by the left and the right-hand sides and the action of the covering projection we conclude that the corresponding equations hold true in $\operatorname{PSL}(2, \mathbb{R})^{\sim}$.

Now we have found that any $\tilde{g} \in P S L(2, \mathbb{R})^{\sim}$ may be written as a product of four translations and four special conformal transformations each of them depending continuously on $\tilde{g}$. Using the results of BorCHERS' construction (lemma II.11), the IwASAWA decomposition and (II.15), (II.16) we define for each $\tilde{g} \in P S L(2, \mathbb{R})^{\sim}$ :

$$
\pi^{\mathcal{A}}(\tilde{g}):=\prod_{i=1}^{4} \widetilde{T}^{\mathcal{A}}\left(p_{\tilde{g}}^{(i)}\right) \widetilde{S}^{\mathcal{A}}\left(n_{\tilde{g}}^{(i)}\right), \quad \tilde{g} \in P S L(2, \mathbb{R})^{\sim}
$$


We have $\pi^{\mathcal{A}}(i d)=\mathbb{1}$. The following lemma asserts that the $\pi^{\mathcal{A}}(\tilde{g})$ define an inner-implementing representation up to a cocycle in the centre of $\mathcal{A}$. To this end we define operators sensitive to the violation of the group multiplication law: $z^{\mathcal{A}}(\tilde{g}, \tilde{h}):=\pi^{\mathcal{A}}(\tilde{g}) \pi^{\mathcal{A}}(\tilde{h}) \pi^{\mathcal{A}}(\tilde{g} \tilde{h})^{*}, \tilde{g}, \tilde{h} \in \operatorname{PSL}(2, \mathbb{R})^{\sim}$.

Lemma II.12: $\pi^{\mathcal{A}}: \tilde{g} \mapsto \pi^{\mathcal{A}}(\tilde{g})$ defines a strongly continuous mapping with unitary values in $\mathcal{A}$. The adjoint action of $\pi^{\mathcal{A}}(\tilde{g}), \tilde{g} \in P S L(2, \mathbb{R})^{\sim}$, on $\mathcal{A}$ implements the automorphism $\alpha_{\tilde{g}}$. $z^{\mathcal{A}}:(\tilde{g}, \tilde{h}) \mapsto z^{\mathcal{A}}(\tilde{g}, \tilde{h})$ defines a strongly continuous 2-cocycle with unitary values in $\mathcal{A}^{\prime} \cap \mathcal{A}$.

Proof: Unitarity is obvious. Strong continuity follows in both cases from continuity of products of continuous functions. The implementing property of the $\pi^{\mathcal{A}}(\tilde{g})$ follows immediately by the decomposition of $\tilde{g}$ into a word containing four translations and four special conformal transformations, the definition of $\pi^{\mathcal{A}}(\tilde{g})$ and lemma II.11 due to BorCHERs. At this point all but the cocycle properties of $z^{\mathcal{A}}$ follow immediately from its definition. If we look at $\pi^{\mathcal{A}}(\tilde{f}) \pi^{\mathcal{A}}(\tilde{g}) \pi^{\mathcal{A}}(\tilde{h})$, insert some identities appropriately, we find: $z^{\mathcal{A}}(\tilde{f}, \tilde{g} \tilde{h}) z^{\mathcal{A}}(\tilde{g}, \tilde{h})=z^{\mathcal{A}}(\tilde{f}, \tilde{g}) z^{\mathcal{A}}(\tilde{f} \tilde{g}, \tilde{h})$. Even more immediate are the equalities $z^{\mathcal{A}}(i d, \tilde{g})=z^{\mathcal{A}}(\tilde{g}, i d)=\mathbb{1}$.

We write the abelian V.NEUMANN algebra generated by the cocycle operators $z^{\mathcal{A}}(\tilde{g}, \tilde{h})$ as follows: $\mathcal{Z}^{\mathcal{A}} \equiv\left\{z^{\mathcal{A}}(\tilde{g}, \tilde{h}), z^{\mathcal{A}}(\tilde{g}, \tilde{h})^{*} \mid \tilde{g}, \tilde{h} \in P S L(2, \mathbb{R})^{\sim}\right\}^{\prime \prime}$. Obviously $\mathcal{Z}^{\mathcal{A}}$ is contained in the centre of $\mathcal{A}$. Now we are prepared to realise the construction itself by proving that the cocycle $z^{\mathcal{A}}$ is exact:

Lemma II.13: For every $\tilde{g} \in P S L(2, \mathbb{R})^{\sim}$ exists a unitary operator $z^{\mathcal{A}}(\tilde{g}) \in \mathcal{Z}^{\mathcal{A}}$ such that

$$
V^{\mathcal{A}}(\tilde{g}):=z^{\mathcal{A}}(\tilde{g}) \pi^{\mathcal{A}}(\tilde{g})
$$

defines a strongly continuous, inner-implementing representation of $P S L(2, \mathbb{R})^{\sim}$.

Proof: As $\mathcal{Z}^{\mathcal{A}} \subset \mathcal{A} \cap \mathcal{A}^{\prime}$ we may apply the direct integral decomposition (cf eg [KR86, chapter 14]). This yields a decomposition of $\mathscr{H}$ as a direct integral of HILBERT spaces $\mathscr{H}_{x}$ and it implies for the operators under consideration: the action of $z^{\mathcal{A}}(\tilde{g}, \tilde{h})$ on $\mathscr{H}_{x}$, denoted by $z^{\mathcal{A}}(\tilde{g}, \tilde{h})(x)$, is a multiple of the identity $\mathbb{1}_{x}$ and thereby defines for almost every $x$ a continuous 2-cocycle $\omega(\tilde{g}, \tilde{h})_{x} \in \mathbb{C}_{1} \mathbb{1}_{x}$. The action of the operators $\pi^{\mathcal{A}}(\tilde{g})$ on $\mathscr{H}_{x}$, denoted by $\pi^{\mathcal{A}}(\tilde{g})(x)$, defines for almost every $x$ a unitary, strongly continuous, projective representation of $P S L(2, \mathbb{R})^{\sim}$.

For LIE groups with a simple LIE algebra the lifting criterion is valid [Sim68], [Jac79, section III.10, theorem 10]. This ensures for almost every $x$ the existence of continuous phases $\omega(\tilde{g})(x)$ such that $\omega(\tilde{g})(x) \pi^{\mathcal{A}}(\tilde{g})(x)$ defines a representation of $\operatorname{PSL}(2, \mathbb{R})^{\sim}$. Integrating $\omega_{x}(\tilde{g})$ over all $x$ yields a unitary $z^{\mathcal{A}}(\tilde{g}) \in \mathcal{Z}^{\mathcal{A}}$, depending strongly continuously on $\tilde{g}$. Integrating the representations defined by the $\omega(\tilde{g})(x) \pi^{\mathcal{A}}(\tilde{g})(x)$ yields a unitary, strongly continuous representation $V^{\mathcal{A}}$ satisfying equation (II.18). $V^{\mathcal{A}}(\tilde{g})$ is an element of $\mathcal{A}$ for every $\tilde{g}$ and implements $\alpha_{\tilde{g}}$ by 
its adjoint action due to lemma II.12.

We summarise the discussions above:

Theorem II.14: Let $\mathscr{H}$ be a separable HILBERT space, and $V$ a representation of $\operatorname{PSL}(2, \mathbb{R})^{\sim}$ on $\mathscr{H}$, unitary, strongly continuous and satisfying the spectrum condition. $\mathcal{A}$ is taken to be a V.NEUMANN algebra of bounded operators on $\mathscr{H}$. The adjoint actions of $V$ on $\mathcal{A}, \mathcal{A}^{\prime}$ shall define groups $\alpha, \alpha^{\prime}$ of automorphisms of $\mathcal{A}, \mathcal{A}^{\prime}$, respectively.

Then there exist unique unitary, strongly continuous, inner-implementing representations $V^{\mathcal{A}}, V^{\mathcal{A}^{\prime}} \equiv V\left(V^{\mathcal{A}}\right)^{*}$ of $\operatorname{PSL}(2, \mathbb{R})^{\sim}$.

Proof: Direct consequence of the propositions and lemmas above.

Two remarks: If we start with a proper representation $V$ of $P S L(2, \mathbb{R})$, then one arrives at representations $V^{\mathcal{A}}, V^{\mathcal{A}^{\prime}}$ which will be (generalized) ray representations of $\operatorname{PSL}(2, \mathbb{R})$ and proper representations of $\operatorname{PSL}(2, \mathbb{R})^{\sim}$. The cocycles of $V^{\mathcal{A}}, V^{\mathcal{A}^{\prime}}$ have to be mutually inverse, and common eigenvectors of $L_{0}^{\mathcal{A}}, L_{0}^{\mathcal{A}^{\prime}}$ have eigenvalues which sum up to integers.

In [Kös02] an alternative derivation is given for $P S L(2, \mathbb{R})$ and $P S O(4,2)$ (conformal group in $3+1$ dimensions) which uses an argument from [BGL95] and should generalise to all conformal groups. This method does not extend to the (universal) covering groups, at least not directly. The approach presented here is close to the one of [BDFS00] for deriving a representation of the POINCARÉ group from modular conjugations of wedge algebras. The corollaries which follow in this section generalise directly to $P S O(4,2)$, as shown in [Kös02].

Now we derive three features of the inner-implementing representations which they inherit from the original representation: spectrum condition, invariant vectors, complete reducibility.

Corollary II.15: Both $V^{\mathcal{A}}$ and $V^{\mathcal{A}^{\prime}}$ satisfy the spectrum condition.

Proof: The operators $V^{\mathcal{A} \vee \mathcal{A}^{\prime}}(\tilde{g}, \tilde{h}):=V^{\mathcal{A}}(\tilde{g}) V^{\mathcal{A}^{\prime}}(\tilde{h})$ define a unitary, strongly continuous representation of $P S L(2, \mathbb{R})^{\sim} \times P S L(2, \mathbb{R})^{\sim}$. With respect to $V^{\mathcal{A} \vee \mathcal{A}^{\prime}}$ we have a dense domain of analytic vectors and we take an arbitrary vector $\psi$ from it. The result follows now as in the proof of proposition II.8 from the inequality

$$
0 \leqslant\left\langle V^{\mathcal{A}}(\widetilde{D}(\tau)) \psi, P V^{\mathcal{A}}(\widetilde{D}(\tau)) \psi\right\rangle=\left\langle\psi, e^{-\tau} P^{\mathcal{A}} \psi\right\rangle+\left\langle\psi, P^{\mathcal{A}^{\prime}} \psi\right\rangle
$$

by letting $\tau \rightarrow \infty$ and $\tau \rightarrow-\infty$, respectively.

Corollary II.16: Let $\Omega \in \mathscr{H}$ be a vector left invariant by $V$. Then $V^{\mathcal{A}}, V^{\mathcal{A}^{\prime}}$ both leave $\Omega$ invariant. 
Proof: Since translations and special conformal transformations generate the whole of $\operatorname{PSL}(2, \mathbb{R})^{\sim}$ it is sufficient to show invariance of $\Omega$ for these two subgroups. We consider translations only; the argument for special conformal transformations is the same.

Take arbitrary $\psi \in \mathscr{H}$. We have $\left\langle\psi, V^{\mathcal{A}}(\tilde{g}) \Omega\right\rangle=\left\langle\psi, V^{\mathcal{A}^{\prime}}(\tilde{g})^{*} \Omega\right\rangle$ by assumption. Set $f_{\psi}(p):=\left\langle\psi, V^{\mathcal{A}}(\widetilde{T}(p)) \Omega\right\rangle, g_{\psi}(p):=\left\langle\psi, V^{\mathcal{A}^{\prime}}(\widetilde{T}(p))^{*} \Omega\right\rangle$. Due to the spectrum condition (corollary II.15) $f_{\psi}$ may be extended to the upper half of the complex plane by means of the LAPLACE transform (cf eg [SW64, chapter 2]). This continuation is analytic in the interior and of at most polynomial growth for complex arguments. On the real line we have $\left|f_{\psi}\right| \leq\|\Omega\|\|\psi\|$ and due to the theorem of Phragmen-LindelöF [Tit39, section 5.62] this bound holds true for the continuation of $f_{\psi}$ as well.

The same line of argument works for $g_{\psi}$ with respect to the lower half of the complex plane. Since $f_{\psi}$ and $g_{\psi}$ coincide on the real line both are restrictions of an entire function (reflection principle). This entire function is bounded by $\|\Omega\|\|\psi\|$, and due to LiouvilLE's theorem it is constant. Since the vectors $V^{\mathcal{A}}(\widetilde{T}(p)) \Omega$, $V^{\mathcal{A}^{\prime}}(\widetilde{T}(p))^{*} \Omega$ are determined by the scalar products $f_{\psi}(p)$ and $g_{\psi}(p), \psi \in \mathscr{H}$, invariance follows by taking $p=0$.

For the next corollary we prepare ourselves by a comment and a lemma. In the corollary the representation $V$ is assumed completely reducible with finite multiplicities. Although this is a pretty strong assumption in group theoretical terms, we consider this a rather convincing assumption from the quantum field theoretical point of view. In this context it is somewhat weaker than a common nuclearity condition [BGL93]. Nuclearity is desirable for quantum field theories and in our setting it corresponds to demanding the $L_{0}$ eigenspaces to be finite-dimensional with degeneracies growing at most exponentially. Typical (integrable) chiral models such as current algebras exhibit this behaviour [GF93]. This implies our assumption as the following lemma clarifies.

The centre of $P S L(2, \mathbb{R})^{\sim}$ is an infinite cyclic group generated by the rotation $\widetilde{R}(2 \pi)$. The following lemma shows that complete reducibility of a representation $V$ as in theorem II.14 is equivalent to requiring the representation space to have a decomposition into a direct sum of eigenspaces of $\widetilde{R}(2 \pi)$. Due to the infinite order of the centre of $\operatorname{PSL}(2, \mathbb{R})^{\sim}$ this is not obvious.

Lemma II.17: Assume $V(\widetilde{R}(2 \pi))$ to have pure point spectrum. Then the spectrum of $L_{0}$ is pure point and $V$ is completely reducible into a direct sum of irreducible representations.

Proof: Let $\mathscr{H}_{i}$ denote the eigenspace belonging to eigenvalue $e^{i 2 \pi h_{i}}$. The restriction of $V(\widetilde{R}(t)) e^{-i h_{i} t}$ to $\mathscr{H}_{i}$ defines a representation of $U(1)$. This representation is completely reducible due to the compactness of $U(1)$ (cf eg [BR77, chapter 
7, $\S 7$, theorem 4 ; chapter $5, \S 4$, proposition 5$]$ ). This proves the claim on the spectrum of $L_{0}$.

By the spectrum condition there are vectors of lowest eigenvalue. Because of the complete analysis of lowest weights in unitary representations of $P S L(2, \mathbb{R})^{\sim}$ [Gri93], it is known which lowest eigenvalues may occur and that the cyclic representations generated from the lowest weight vectors are irreducible. Taking such a lowest weight vector, applying to it the linear span of the $V(\tilde{g}), \tilde{g} \in P S L(2, \mathbb{R})^{\sim}$, and taking the completion yields an irreducible representation space. We may reduce with respect to it because of unitarity. We iterate this procedure and arrive at the second claim since $\mathscr{H}$ is separable.

Corollary II.18: Assume $V$ to be completely reducible with finite multiplicities. Then $V^{\mathcal{A}}$ and $V^{\mathcal{A}^{\prime}}$ are completely reducible.

Proof: Denote the lowest weight vectors by $\varphi_{(d, i)}, i$ being the multiplicity index and $d$ the eigenvalue of $L_{0}$. For any fixed $d$ the $\varphi_{(d, i)}$ span a finite-dimensional HiLBERT space. This space is left invariant by the operators $V^{\mathcal{A}}(\widetilde{R}(2 \pi)), V^{\mathcal{A}^{\prime}}(\widetilde{R}(2 \pi))$. Both operators may be diagonalised on this space simultaneously, the result being a mere relabelling of the irreducible subrepresentations of $V$. Now $V^{\mathcal{A}}(\widetilde{R}(2 \pi))$, $V^{\mathcal{A}^{\prime}}(\widetilde{R}(2 \pi))$ both are diagonal on the irreducible subspaces generated from the "new" lowest weight vectors $\varphi_{(d, i)}^{\prime}$ and thus on the whole of $\mathscr{H}$. Now the claim follows as in the proof of lemma II.17.

Remark: Non-trivial unitary representations of $P S L(2, \mathbb{R})^{\sim}$ are necessarily infinitedimensional and the multiplicity spaces of $V^{\mathcal{A}}$ serve as representation spaces for $V^{\mathcal{A}^{\prime}}$ and vice versa. The irreducible representations of $V^{\mathcal{A}}$ and $V^{\mathcal{A}^{\prime}}$ will, therefore, not have finite multiplicities in general.

\subsection{Direct applications to chiral subnets}

We collect a few immediate implications of the BORCHERS-SUGAWARA construction for chiral subnets $\mathcal{A} \subset \mathcal{B}$.

Proposition II.19: The inner-implementing unitaries $U^{\mathcal{A}}(g) \neq \mathbb{1}$ are not elements of any local algebra. If $\mathcal{A} \neq \mathbb{C} \mathbb{1}$, then $\mathcal{A}$ contains non-trivial non-local operators, the vacuum is not faithful for $\mathcal{A}$, and the action of $A d_{U \mathcal{A}}$ on the local operators of the net $\mathcal{A}$ is ergodic.

Proof: Suppose that for some $\tilde{g} \in P S L(2, \mathbb{R})^{\sim}$ the unitary $U^{\mathcal{A}}(\tilde{g}) \neq \mathbb{1}$ is contained in a local algebra. By locality and invariance of the vacuum there is a local algebra $\mathcal{B}(I)$ such that all vectors $B \Omega, B \in \mathcal{B}(I)$, remain unchanged when acted upon by $U^{\mathcal{A}}(\tilde{g})$. Thus, by the REEH-SCHLIEDER property of $\mathcal{B}, U^{\mathcal{A}}(\tilde{g})$ has to be trivial and the existence of such operators is denied. 
The kernel of $U^{\mathcal{A}}$ has to be different from $P S L(2, \mathbb{R})^{\sim}$, else $\mathcal{A}$ is left invariant point-wise by the covariance automorphisms and therefore must be abelian by locality. But local algebras of $\mathcal{A}$ have to be factors as elements of a chiral subnet. So $\mathcal{A} \neq \mathbb{C} \mathbb{1}$ requires the existence of operators $U^{\mathcal{A}}(\tilde{g}) \neq \mathbb{1}$. These are not local operators.

Any fixed point of the action of $A d_{U_{\mathcal{A}}}$ on a local algebra $\mathcal{A}(I)$ has to be contained in its centre due to locality. This centre is trivial since $\mathcal{A}(I)$ is a factor. $\Omega$ can not be separating, because we have: $\left(U^{\mathcal{A}}(\tilde{g})-\mathbb{1}\right) \Omega=0$.

The inversion on the light-ray, $I_{+}: x \mapsto-x \in \mathbb{R}$, induces an outer automorphism of $P S L(2, \mathbb{R}): \eta(g)=I_{+} g I_{+}$. We have in particular: $\eta(T(a))=T(-a)$, $\eta(D(t))=D(t), \eta(S(n))=S(-n), \eta(R(\varphi))=R(-\varphi)$. Obviously, this outer automorphism extends to the universal covering group, where we denote it by $\tilde{\eta}$. We have for the covering projection $\mathrm{p}$ : $\mathrm{p} \tilde{\eta}=\eta \mathrm{p}$, which may be checked directly eg by looking at the IWASAWA decomposition.

The Bisognano-Wichmann property of $\mathcal{B}$ implies that the modular conjugation of $\mathcal{B}\left(\mathrm{S}_{+}^{1}\right)$, namely $J$, implements $I_{+}$. By this, the representation $U$ and the operator $J$ generate a representation of $P S L(2, \mathbb{R})_{ \pm} \equiv P S L(2, \mathbb{R}) \rtimes_{\eta} \mathbb{Z}_{2}$, the group generated by $I_{+}$and $P S L(2, \mathbb{R})$ modulo the relation $\eta(g)=I_{+} g I_{+}$. The following simple proposition shows, that $J$ and $U^{\mathcal{A}}$ together generate a representation of $P S L(2, \mathbb{R})_{ \pm}^{\sim}$.

Proposition II.20: The adjoint action of the modular conjugation $J$ of $\mathcal{B}\left(\mathrm{S}_{+}^{1}\right)$ on $U^{\mathcal{A}}$ implements the outer automorphism $\tilde{\eta}$, ie we have: $J U^{\mathcal{A}}(\tilde{g}) J=U^{\mathcal{A}}(\tilde{\eta}(\tilde{g}))$. The same is true for $U^{\mathcal{A}^{\prime}}$.

Proof: $J U^{\mathcal{A}} J$ defines a globally inner representation of $P S L(2, \mathbb{R})^{\sim}$, since $A d_{J}$ defines automorphisms of the global algebra $\mathcal{A}$. For arbitrary $A \in \mathcal{A}(I), I \Subset \mathrm{S}^{1}$, we set $A^{\prime}:=J A J$. This allows us to write:

$$
A d_{J U^{\mathcal{A}}(\tilde{g}) J}(A)=A d_{J}\left(\alpha_{\mathrm{p}(\tilde{g})}\left(A^{\prime}\right)\right)=\alpha_{\eta(\mathbf{p}(\tilde{g}))}(A) .
$$

Thus, $J U^{\mathcal{A}} J$ implements an automorphic action $\alpha \circ \eta$ of $\operatorname{PSL}(2, \mathbb{R})$ on $\mathcal{A}$. Conversely, $J U^{\mathcal{A}}(\tilde{\eta}())$.$J implements the automorphic action \alpha$, is a globally inner representation of $\operatorname{PSL}(2, \mathbb{R})^{\sim}$, and hence identical to the unique inner-implementing representation $U^{\mathcal{A}}$ (proposition II.9). This proves the statement for $U^{\mathcal{A}}$. A look at the very definition of $U^{\mathcal{A}^{\prime}}$ completes the proof.

In a large class of chiral conformal models such as free fermions and chiral current algebras there are explicit constructions for the transformation operators as observables in terms of local quantum fields (cf eg [FST89] and chapter III). In both cases the construction yields a representation of the whole VIRASORO algebra. This diffeomorphism invariance is necessarily broken in any positiveenergy representation; it remains a $\operatorname{PSL}(2, \mathbb{R}) \sim$ symmetry only. 
We have constructed the inner implementation of this remaining symmetry in a completely model independent way and used the result in lemma II.4 for the definition of the maximal COsET model $\mathcal{C}_{\max }$ associated with a given subnet $\mathcal{A} \subset \mathcal{B}$. It might happen that a subnet $\mathcal{A} \subset \mathcal{B}$ admits no Coset theory at all:

Definition II.21: A chiral subnet $\mathcal{A} \subset \mathcal{B}$ is called a conformal inclusion, if $\mathcal{C}_{\text {max }}(I)=\mathbb{C} \mathbb{1}, I \Subset \mathrm{S}^{1}$.

This term stems from studies on chiral current algebras. Here we have for both nets $\mathcal{A} \subset \mathcal{B}$ stress-energy tensors $\Theta^{\mathcal{A}}, \Theta^{\mathcal{B}}$. A simple argument shows that their difference $\Theta^{\mathcal{B}}-\Theta^{\mathcal{A}} \equiv \Theta^{\text {coset }}$ is a stress energy tensor alike. By the REEHSCHLIEDER theorem and the LÜSCHER-MACK theorem [FST89, Mac88, LM76] $\Theta^{\text {coset }}$ vanishes iff its central charge vanishes. Its central charge is completely determined by the finite-dimensional LIE algebras from which the current algebras are constructed and by the embedding of the smaller one into the larger one. Its zeros, characterising the notion of conformal embeddings for these models, have been classified [SW86, AGO87, BB87].

In section III. 4 we give a characterisation of conformal inclusions of current algebras by methods familiar in axiomatic quantum field theory and, thus, giving an illustration of our perspective to look at such problems by analysing the action of the inner implementation of covariance of the subnet on the larger theory. This approach avoids arithmetic considerations on the central charge.

The following proposition shows that our definition of conformal inclusions covers conformal inclusions of current algebras (understood as above) as special cases (cf theorem III.3). Chiral subnets of finite index are necessarily conformal inclusions in the sense of definition II.21 (lemma V.1).

Proposition II.22: Suppose the inner-implementing representation of theorem II.14 for a chiral subnet $\mathcal{A} \subset \mathcal{B}$ satisfies $U=U^{\mathcal{A}}$. Then $\mathcal{A} \subset \mathcal{B}$ is conformal.

Proof: By assumption we have $U^{\mathcal{A}^{\prime}}=\mathbb{1}$. Since $U^{\mathcal{A}^{\prime}}$ implements covariance on any Coset theory, the local algebras of $\mathcal{C}_{\max }$ have to be trivial by the reasoning given in the proof of proposition II.19.

Looking at the factorisation $U \circ \mathrm{p}=U^{\mathcal{A}} U^{\mathcal{A}^{\prime}}$ and the spectrum condition of all three of them (theorem II.14, corollary II.15), this proposition says that one can not have non-trivial COSET models if all the energy of $\mathcal{B}$, represented in a global sense by $U$, already belongs to $\mathcal{A}$, ie if $U^{\mathcal{A}^{\prime}}=\mathbb{1}$. Hence, we are interested in situations where not all of the "energy content" of $\mathcal{B}$ is contained in $\mathcal{A}$, as mentioned in the introduction (chapter I).

It is not clear in general whether $U^{\mathcal{A}^{\prime}}$ is globally inner in $\mathcal{C}_{\text {max }}$. It is, of course, if both $\mathcal{B}$ and $\mathcal{A}$ possess a stress-energy tensor, in which case $U^{\mathcal{A}^{\prime}}$ is generated by the Coset stress-energy tensor (theorem III.3). If $U^{\mathcal{A}^{\prime}}$ is contained in $\mathcal{C}_{\text {max }}$ and we apply the BORCHERS-SUGAWARA construction with respect to the action 
of $U$ on $\mathcal{C}_{\text {max }}$, yielding the representation $U^{\mathcal{C}_{\text {max }}}$, then we have: $U=U^{\mathcal{A}} U^{\mathcal{C}_{\text {max }}}$. This identity is the complete analogue of the decomposition of the stress energy tensor $\Theta^{\mathcal{B}}$ of an ambient theory into the stress-energy tensor $\Theta^{\mathcal{A}}$ of a subtheory and the respective Coset stress-energy tensor $\Theta^{\mathcal{B}}-\Theta^{\mathcal{A}}$. Probably, this equality holds true in general, but we know of no proof to date.

Taking this lack of understanding seriously, one is led to the following consideration: If we iterate the BORCHERS-SUGAWARA construction with respect to the action of $U^{\mathcal{A}^{\prime}}$ on $\mathcal{C}_{\text {max }}$, and with respect to the action of $U^{\mathcal{C}_{\text {max }}^{\prime}}$ on $\mathcal{A}$, we get the inner-implementing representations $U^{\mathcal{C}_{\text {max }}}$ and $U^{\mathcal{A}}$ and the respective remainders $U_{\left(\mathcal{A}^{\prime}\right)}^{\mathcal{C}_{\text {max }}^{\prime}}, U_{\left(\mathcal{C}_{\text {max }}^{\prime}\right)}^{\mathcal{A}^{\prime}}$ such that we have the identities:

$$
U^{\mathcal{A}^{\prime}}=U^{\mathcal{C}} U_{\left(\mathcal{A}^{\prime}\right)}^{\mathcal{C}_{\text {max }}^{\prime}}, \quad U^{\mathcal{C}_{\text {max }}^{\prime}}=U^{\mathcal{A}} U_{\left(\mathcal{C}_{\max }^{\prime}\right)}^{\mathcal{A}^{\prime}}
$$

Obviously, $U^{\mathcal{A}}, U^{\mathcal{C}}$ commute with each other and with $U_{\left(\mathcal{A}^{\prime}\right)}^{\mathcal{C}_{\text {max }}^{\prime}}, U_{\left(\mathcal{C}_{\text {max }}^{\prime}\right)}^{\mathcal{A}^{\prime}}$ and, moreover, the following holds:

$$
U \circ \mathrm{p}=U^{\mathcal{A}} U^{\mathcal{C}} U_{\left(\mathcal{A}^{\prime}\right)}^{\mathcal{C}_{\text {max }}^{\prime}}=U^{\mathcal{C}} U^{\mathcal{A}} U_{\left(\mathcal{C}_{\max }^{\prime}\right)}^{\mathcal{A}^{\prime}}
$$

Thus, $U_{\left(\mathcal{A}^{\prime}\right)}^{\mathcal{C}_{\text {max }}^{\prime}}$ and $U_{\left(\mathcal{C}_{\max }^{\prime}\right)}^{\mathcal{A}^{\prime}}$ coincide with the remainder of the BorCHERS-SUGAWARA construction on the Coset pair $\mathcal{A} \subset \mathcal{C}_{\max } \subset \mathcal{B}$, namely:

$$
U_{\left(\mathcal{A}^{\prime}\right)}^{\mathcal{C}_{\text {max }}^{\prime}}=U_{\left(\mathcal{C}_{\text {max }}^{\prime}\right)}^{\mathcal{A}^{\prime}}=U^{\mathcal{A}^{\prime} \cap \mathcal{C}_{\max }^{\prime}}=U \circ \mathrm{p}\left(U^{\mathcal{A}}\right)^{*}\left(U^{\mathcal{C}_{\text {max }}}\right)^{*}
$$

As a next step one may observe that the inclusion $\mathcal{A} \subset \mathcal{C}_{\max } \subset \mathcal{B}$ is conformal: the local operators of its maximal Coset theory have to be central in the re-

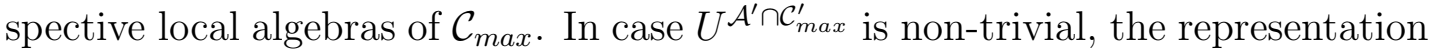
$U$ can not be generated by local data according to the scheme indicated (II.20), ie the result would exhibit a kind of commutativity of its constituents which its local approximations can not have. This appears unsatisfactory.

It is straightforward to check that in case $\mathcal{A} \subset \mathcal{B}$ is cofinite and both $U^{\mathcal{A}}$ and $U^{\mathcal{C}_{\max }}$ have the net-endomorphism property (definition IV.1) the representation $U^{\mathcal{A}^{\prime} \cap \mathcal{C}_{\text {max }}^{\prime}}$ has to be trivial (proposition $\mathrm{V} .2$ ). This result relies on a purely representation theoretic argument concerning the action of $P S L(2, \mathbb{R}) \sim$ on finitedimensional subspaces of a unitary representation, which, basically, trivialises the problem. A general argument based on fundamental assumptions such as the split property is highly desirable, but out of reach to date.

Therefore, it is not known whether our definition of conformal inclusion, referring to triviality of all CoseT models associated with $\mathcal{A} \subset \mathcal{B}$, actually is equivalent to $U=U^{\mathcal{A}}$. For this work there is no need to go into this problem in detail, but we would like to state which extended Coset pair might be attached to the possibility $U^{\mathcal{A}^{\prime} \cap \mathcal{C}_{\text {max }}^{\prime}} \neq \mathbb{1}$.

The maximal iterated Coset model, $\mathcal{A}_{2}$, associated with $\mathcal{C}_{\text {max }} \subset \mathcal{B}$ is given by the local algebras $\mathcal{A}_{2}(I):=\left\{U^{\mathcal{C}_{\max }}\right\}^{\prime} \cap \mathcal{B}(I)$. It contains the subnet $\mathcal{A}_{\text {max }}$ 
consisting of all observables which are covariant with respect to $U^{\mathcal{A}}$, ie commute with $U^{\mathcal{A}^{\prime}}$ :

$$
\mathcal{A}_{\text {max }}(I):=\left\{U^{\mathcal{A}^{\prime}}(g), g \in P S L(2, \mathbb{R})^{\sim}\right\}^{\prime} \cap \mathcal{B}(I)
$$

This is obvious since $\mathcal{A}_{\text {max }}$ is a Coset model associated with $\mathcal{C}_{\text {max }}$ (lemma II.4). Clearly, $\mathcal{A}_{2}$ and $\mathcal{C}_{\max }$ can not be extended without spoiling their character as a Coset pair.

As argued above, we expect $U=U^{\mathcal{A}} U^{\mathcal{C}_{\text {max }}}$, which yields $\mathcal{A}_{2}=\mathcal{A}_{\text {max }}$. We take $\mathcal{A}_{\text {max }}$ to be the other natural object in our studies of chiral subnets and their Coset models. To put it plainly: We do not assume $U^{\mathcal{A}^{\prime}}=U^{\mathcal{C}_{\max }}$ to hold, we rather proceed without having an answer to this problem. In all what follows, our analysis will be done in terms of $U^{\mathcal{A}}$ and $U^{\mathcal{A}^{\prime}}$ and we will not have to face again the questions just raised, because the forthcoming arguments will be independent. The further development leads to satisfactory results, which support our opinions on this issue.

We mention another reason for considering $\mathcal{A}_{\text {max }}$ as a natural object of investigation. While given $\mathcal{A}$ and $U$ the inner-implementing representation $U^{\mathcal{A}}$ is unique, $U^{\mathcal{A}}$ does not determine the subnet $\mathcal{A} \subset \mathcal{B}$, as examples of conformal inclusions show. In general there will be subnets transforming covariantly under the action of $U^{\mathcal{A}}$ (transformation property) and subnets containing the operators of $U^{\mathcal{A}}$ as global observables (generating property). Generically there will be no simple relation such as inclusion or commutativity etc for any pair $\mathcal{A}_{\alpha}, \mathcal{A}_{\beta}$ of chiral subnets having one or both properties. $\mathcal{A}_{\text {max }}$ is, of course, the maximal subnet transforming covariantly and having the generating property. Any subnet $\mathcal{A}$ having both properties defines a conformal inclusion $\mathcal{A}_{\mathcal{A}_{\text {max }}} \subset \mathcal{A}_{\text {max }} e_{\mathcal{A}_{\text {max }}}$. Since studies on conformal inclusions form an area of research of their own, $\mathcal{A}_{\max }$ should be a generic object to explore.

\subsection{Discussion on the BORCHERS-SUGAWARA construction}

We have presented a construction applying and generalising the result of BorCHERS [Bor66]. Hence we obtained the unique inner-implementing representation of $\operatorname{PSL}(2, \mathbb{R})^{\sim}$. It generalises, within its limits, the SUGAWARA construction [Sug68]. We have proposed the name Borchers-Sugawara construction because of these relations. The construction is completely model independent and does not require existence of a stress-energy tensor. Properties connected with representations generated by a stress-energy tensor will be discussed in IV.

It is natural to ask if this construction may be applied to other spacetime symmetry groups. In our view the key tools in our construction are the following: The original representation satisfies the spectrum condition for some translation subgroups. There are sufficiently many of them to generate the whole group and we have an argument how to derive a representation of the covering group from the unitary group generated by the operators constructed by means of BorCHERs' 
key result [Bor66].

In the case of the POINCARÉ group the translations usually satisfy the spectrum condition. Unfortunately, so to say, they form an invariant subgroup and although one is tempted to generate the group from $P S L(2, \mathbb{R})$ subgroups (as eg in [KW01]) this seems impossible with subgroups satisfying the spectrum condition.

For conformal groups the situation is different, as shown in [Kös02]. These groups are generated by their subgroups of translations and special conformal transformations [BGL93] and both subgroups satisfy the spectrum condition, if the conformal Hamiltonian has positive spectrum. The proof of main theorem in [Kös02] (corresponding to theorem II.14) extends to all conformal groups $P S O(d, 2), d \geq 3$, and the construction given here applies directly to the conformal group in $1+1$ dimensions, since this is a factor group of $\operatorname{PSL}(2, \mathbb{R})^{\sim} \times$ $\operatorname{PSL}(2, \mathbb{R})^{\sim}$.

The construction used in [Kös02], however, does not extend to coverings of the conformal groups, apparently. Applying the method used here requires establishing an explicit, continuous decomposition into translations and special conformal transformations, which ought to be possible. 


\section{Chapter III}

\section{Subnets of chiral current algebras}

The purpose of this chapter is to introduce the objects of most investigations of chiral subnets and their CoseT models: the chiral conformal theories and subtheories generated by chiral current algebras. These models are either formulated as theories of WIGHTMAN fields in $1+1$ dimensions, which factorise into independent chiral parts, or more abstractly through unitarisable highest-weight representations of affine Kac-Moody algebras, which then are shown to integrate to chiral conformal nets by group theoretical methods and can be seen to define chiral conformal quantum field theories in the sense of WiGHTMAN's axioms as well.

If one wants to study model specific properties of current algebras, their superselection structure in particular, other approaches to constructing chiral current algebras prove very useful. They may be defined by the action of BogoluUBOv (gauge) transformations on the CAR algebra of chiral fermions [Was98] or one may use the basic construction of FRENKEL-KAC [FK80] for affine KAC-MoODY algebras $A_{n}^{(1)}, D_{n}^{(1)}, E_{6-8}^{(1)}$ at level 1, using vertex operators (see eg [TL97]). Closely related to the latter point of view, one may consider these models as local extensions of the rank $_{\mathfrak{g}}$-fold tensor product of the $U(1)$-current algebra (eg [Sta95]); a variant is the approach of [GF93].

Several authors have dealt with specific properties of chiral current algebras, their inclusions and their Coset models (eg [Xu00a, Xu99, Xu00b, Xu01, Xu02, KL02, TL97, Was98, Lok94]). We are primarily interested in the archetypical, instructive character of these models and for this reason we focus on establishing the properties needed in the definitions II.1 and II.2 and additional properties connected with the Sugawara construction, which ensures presence of a stressenergy tensor in any chiral current algebra.

Therefore, we take a straightforward approach to these models through a construction using free, chiral fermion fields, the quarks, take a direct route from the Wightman fields to the chiral conformal theory they generate, and discuss other aspects of these models later on. The term chiral current algebra will be used for all these models. 


\section{Quark models of chiral current algebras}

First, we describe how chiral current algebras may be obtained directly as quantum fields in the sense of WightMAN's axioms from free, massless fermion fields, the quarks, a method introduced in [BH71]. The SUGAWARA construction for chiral currents, which yields a stress-energy tensor for each chiral current algebra, is discussed as the next step.

The fundamental objects of the framework laid down in WighTMAN's axioms are local quantum fields, $\Phi$, which consist of maps from smooth test functions on spacetime, $f$, to (generically unbounded) closable, linear operators, $\Phi(f)$, defined on a dense subspace of some HiLBERT space. The fields $\Phi$ usually carry multiple indices referring to their type, in particular to their transformation behaviour under gauge and spacetime transformations. Covariance with respect to spacetime symmetries is required to be implemented by a unitary, strongly continuous representation which satisfies the spectrum condition and admits a unique (up to its phase) normalised, invariant vector, the vacuum $\Omega$. Multiple application of smeared fields $\Phi(f)$ to the vacuum, $\Omega$, is assumed to generate a dense subspace, the Wightman domain, which is common to all smeared fields, invariant under their action and allows their reconstruction, ie is a core for all fields. The matrix elements of the fields $\Phi(f)$ have to depend continuously on the test function $f$, ie they shall be tempered distributions. Locality is formulated as (graded) commutativity of the smeared fields $\Phi_{1}\left(f_{1}\right), \Phi_{2}\left(f_{2}\right)$ on the Wightman domain for test functions $f_{1}, f_{2}$ which have spacelike separated supports. According to the Wightman reconstruction theorem [Wig56], the whole theory including its covariance with respect to a unitary implementation of spacetime and gauge symmetry is encoded in the properties of the hierarchy of $n$-point functions, namely in the numerical, tempered distributions defined by the vacuum expectation values of products of fields as eg $\left\langle\Omega, \Phi_{1}\left(f_{1}\right) \ldots \Phi_{n}\left(f_{n}\right) \Omega\right\rangle$.

Reviews and textbooks on this framework of relativistic quantum field theory are eg [WG65, SW64, Jos65, Tod65], a short summary is contained in R. HAAG's book [Haa92, sections II.1, II.2]. The discussion in sections 1.1 and 1.2 is a summary of well-known results. The review of FurLan, SotKOV AND TODOROV [FST89] serves as a source for the formulation of the general theory of quantised fields with conformal symmetry in $1+1$ dimensions, and [GO86, Fuc92] contain expositions of quark models and compact LIE algebras.

\subsection{Chiral currents as WICK squares}

Take $N$ independent, free, massless, complex fermion fields, the quarks $\psi^{i}, i=$ $1, \ldots, N$, in $1+1$ dimensions. They satisfy the corresponding Dirac equation:

$$
i \not \partial \psi^{i}(t, x)=i\left(\gamma^{0} \partial_{0}+\gamma^{1} \partial_{1}\right) \psi^{i}=0 .
$$


Choosing units in which the speed of light equals $1, t=x^{0}$ stands for the time coordinate and $x=x^{1}$ for the space coordinate. The algebra of the $\gamma$-matrices is given by $\left(\gamma^{0}\right)^{2}=\mathbb{1}=-\left(\gamma^{1}\right)^{2},\left\{\gamma^{0}, \gamma^{1}\right\}=0$. With $\gamma^{5}:=\gamma^{0} \gamma^{1}$ and $P_{ \pm}:=$ $\frac{1}{2}\left(1 \pm \gamma^{5}\right)$ the quarks can be decomposed into their chiral components, each of which depends on one light-cone coordinate only:

$$
\left(\partial_{0} \pm \partial_{1}\right) P_{ \pm} \psi^{i}(t, x)=0 \Leftrightarrow \psi_{ \pm}^{i}(t \mp x):=P_{ \pm} \psi^{i}(t, x)
$$

For the time being we use the light-cone coordinates $x_{ \pm}:=t \mp x$.

The quantum field theoretical nature of these fields is encoded in canonical anti-commutation relations (CAR):

$$
\begin{aligned}
\left\{\psi_{+}^{i}\left(x_{+}\right), \psi_{-}^{j}\left(y_{-}\right)\right\} & =\left\{\psi_{+}^{i}\left(x_{+}\right), \psi_{-}^{j}\left(y_{-}\right)^{*}=0\right. \\
\left\{\psi_{+}^{i}\left(x_{+}\right), \psi_{+}^{j}\left(y_{+}\right)\right\} & =\left\{\psi_{-}^{i}\left(x_{-}\right), \psi_{-}^{j}\left(y_{-}\right)\right\}=0 \\
\left\{\psi_{+}^{i}\left(x_{+}\right), \psi_{+}^{j}\left(y_{+}\right)^{*}\right\} & =\delta\left(x_{+}-y_{+}\right) \delta^{i j} \\
\left\{\psi_{-}^{i}\left(x_{-}\right), \psi_{-}^{j}\left(y_{-}\right)^{*}\right\} & =\delta\left(x_{-}-y_{-}\right) \delta^{i j} .
\end{aligned}
$$

The non-vanishing two-point functions of these fields are given by:

$$
\left\langle\Omega, \psi_{ \pm}^{i}\left(x_{ \pm}\right) \psi_{ \pm}^{j}\left(y_{ \pm}\right)^{*} \Omega\right\rangle=\left\langle\Omega, \psi_{ \pm}^{i}\left(x_{ \pm}\right)^{*} \psi_{ \pm}^{j}\left(y_{ \pm}\right) \Omega\right\rangle=\frac{\delta^{i j}}{2 \pi} \Delta\left(x_{ \pm}-y_{ \pm}\right) .
$$

The distribution $\Delta(t)$ is given as boundary value of the analytic function $\frac{-i}{t-i \varepsilon}$ in the limit $\varepsilon \searrow 0$.

The CAR determine the $n$-point functions completely in terms of the twopoint functions and it is well-known that fermion fields as above define quantised fields satisfying Wightman's axioms on the fermionic FoCK space (see eg [Jos65, section II.5]). The transformation behaviour of $\Delta$ with respect to coordinate transformations shows that conformal symmetry is present, implemented by a unitary representation $U_{\psi}$ of the twofold covering of $\left(P S L(2, \mathbb{R})^{\sim} \times\right.$ $\left.\operatorname{PSL}(2, \mathbb{R})^{\sim}\right) / \mathbb{Z}$, where the cyclic group generated by the simultaneous rigid conformal rotations by $2 \pi, \tilde{R}(2 \pi) \times \tilde{R}(2 \pi)$, is factored out. Moreover, the decomposition into chiral components (III.1) yields a decomposition of the WIGHTMAN theory of quarks $\psi^{i}$ on fermionic Fock space, $\mathscr{H}_{\psi}$, into the tensor product of the Wightman theories of the chiral quarks $\psi_{ \pm}^{i}: \mathscr{H}_{\psi}=\mathscr{H}_{\psi_{+}} \otimes \mathscr{H}_{\psi_{-}}$.

The quarks $\psi^{i}$ are found to be quasi-primary fields of scaling dimension $\frac{1}{2}$, ie they have a definite transformation behaviour determined by the scaling dimension, which we state for one chiral component:

$$
U_{\psi}(\tilde{g}) \psi_{+}^{i}\left(x_{+}\right) U_{\psi}(\tilde{g})^{*}=(d(\mathrm{p}(\tilde{g})(x)) / d x)^{\frac{1}{2}} \psi_{+}^{i}\left(\mathrm{p}(\tilde{g})\left(x_{+}\right)\right), \tilde{g} \in P S L(2, \mathbb{R})^{\sim} .
$$

For the transformation law of a general quasi-primary field of scaling dimension $h$ replace $\frac{1}{2}$ by $h$.

The normal ordering of free complex fermions is given by the usual pointsplitting procedure:

$$
: \psi_{+}^{i} \psi_{+}^{j *}:\left(x_{+}\right)=\lim _{x_{+}^{\prime} \rightarrow x_{+}}\left(\psi_{+}^{i}\left(x_{+}\right) \psi_{+}^{j}\left(x_{+}^{\prime}\right)^{*}-\frac{\delta^{i j}}{2 \pi} \Delta\left(x_{+}-x_{+}^{\prime}\right)\right) .
$$


It is common to call this Wightman field the Wick square of the free complex fermions.

Now, we assume that there is an $N$-dimensional, non-trivial representation of a compact, simple LIE algebra $\mathfrak{g}$ by Hermitian matrices $\left\{\left(M_{i j}^{a}\right)_{i, j=1, \ldots, N}\right\}_{a=1, \ldots, d_{\mathfrak{g}}}$, where $d_{\mathfrak{g}}$ denotes the dimension of $\mathfrak{g}$. This means that we have: $\overline{M_{i j}^{a}}=M_{j i}^{a}$ and $\left[M^{a}, M^{b}\right]=i{ }^{a b}{ }_{c} M^{c}$. The real numbers $f^{a b}{ }_{c}$ are the structure constants of $\mathfrak{g}$ defined with respect to some basis $\left\{T^{a}\right\}_{a=1, \ldots, d_{\mathfrak{g}}}$. The 1+1-dimensional complex quark model of the current algebra associated with $\mathfrak{g}$ is given as follows:

$$
j^{a \mu}(t, x):=M_{i j}^{a}: \overline{\psi^{i}} \gamma^{\mu} \psi^{j}:(t, x)=M_{i j}^{a}: \psi^{i *} \gamma^{0} \gamma^{\mu} \psi^{j}:(t, x) .
$$

It is straightforward to check that this defines a Hermitian field of scaling dimension 1 and that it is a conserved current: $\partial_{\mu} j^{a \mu}=0$.

$j^{a \mu}$ decomposes into two independent chiral parts, the chiral currents $j_{ \pm}^{a}$ :

$$
j_{ \pm}^{a}:=\frac{1}{2}\left(j^{a 0} \pm j^{a 1}\right)=M_{i j}^{a}: \psi^{i *} P_{ \pm} \psi^{j}:=M_{i j}^{a}: \psi_{ \pm}^{i *} \psi_{ \pm}^{j}:
$$

The decomposition of the fermionic Fock space into the tensor product of chiral fermionic FocK spaces induces a tensor-product decomposition of the HILBERT space which the currents generate from the vacuum, $\mathscr{H}_{j}$, into the tensor product of the vacuum HiLBERT spaces of the chiral currents: $\mathscr{H}_{j}=\mathscr{H}_{j_{+}} \otimes \mathscr{H}_{j_{-}}$. The chiral currents act as $j_{+}^{a}=j_{+}^{a} \otimes \mathbb{1}_{j_{-}}, j_{-}^{a}=\mathbb{1}_{j_{+}} \otimes j_{-}^{a}$.

The commutation relations between chiral currents read:

$$
\left[j_{ \pm}^{a}\left(x_{ \pm}\right), j_{ \pm}^{b}\left(y_{ \pm}\right)\right]=i f^{a b}{ }_{c}^{c} j_{ \pm}^{c}\left(y_{ \pm}\right) \delta\left(x_{ \pm}-y_{ \pm}\right)+\operatorname{tr}\left(M^{a} M^{b}\right) \frac{i}{2 \pi} \delta^{\prime}\left(x_{ \pm}-y_{ \pm}\right) .
$$

The currents are examples of Lie fields, as their commutator is linear in the field itself. In higher dimensions the possibilities of LIE fields are very restricted (see eg [Bau76] and references therein). The $c$-number part in (III.5) is called the Schwinger term.

The trace $\operatorname{tr}\left(M^{a} M^{b}\right)$ is invariant under cyclic permutations and hence induces an invariant symmetric bilinear form on $\mathfrak{g}$. Since $\mathfrak{g}$ is simple, there is up to normalisation only one such form and thus $\operatorname{tr}\left(M^{a} M^{b}\right)$ has to be proportional to the trace in the adjoint representation of $\mathfrak{g}$. The latter is usually normalised such that the highest weight of the adjoint representation has length 2 in the scalar product which is induced by the form. Because $\mathfrak{g}$ is compact, the result is a Euclidean scalar product which we will call the Killing form of $\mathfrak{g}$.

The KILLING form will be denoted $\langle., .\rangle_{\mathfrak{g}}$ and the matrix which induces it will be written $g_{\mathfrak{g}}^{a b}$. The inverse of $g_{\mathfrak{g}}^{a b}$ is written with two lower indices: $g_{a b}^{\mathfrak{g}}$. With these conventions, there exists a positive integer $k$ such that $\operatorname{tr}\left(M^{a} M^{b}\right)=k g_{\mathfrak{g}}^{a b}$. $k$ is the second Dynkin index of the representation of $\mathfrak{g}$ by the matrices $M^{a}$ and in relation to the commutation relations (III.5) it is the level of the current algebra associated with $\mathfrak{g}$. 
The constant functions on the light-cone are among the admissible test functions for currents (cf below). If one smears out currents with constant test functions, one reads off the current algebra (III.5) that such current operators form a representation of $\mathfrak{g}$, which is contained in the current algebra and is commonly called the horizontal subalgebra. The adjoint action of the horizontal subalgebra on quarks and on currents generates global gauge transformations of both fields and thus the currents may be viewed as conserved currents associated with a gauge symmetry. For this reason we regard the label " $a$ " of the currents $j_{ \pm}^{a}$ as their colour and will call the algebra of the $T^{a}$, the LIE algebra $\mathfrak{g}$, the colour algebra of our current algebra. The colour algebra and the level characterise a current algebra completely.

For orthogonal representations, there is the real quark model. We assume there are $N$-dimensional, skew-symmetric, real matrices $M^{a}$ forming a non-trivial, orthogonal representation of $\mathfrak{g}$. Then we take $N$ real fermions $\psi_{r}^{i}(t, x):=\frac{1}{2}\left(\psi^{i}(t, x)+\right.$ $\left.\psi^{i}(t, x)^{*}\right)$. It is readily checked that the following definition yields a current algebra associated with $\mathfrak{g}$ at the level $k$ satisfying $k g_{\mathfrak{g}}^{a b}=\frac{1}{2} \operatorname{tr}\left(M^{a} M^{b}\right)$ :

$$
j^{a \mu}(t, x)=\left(i M_{i j}^{a}\right): \psi_{r}^{i} \gamma^{0} \gamma^{\mu} \psi_{r}^{j}:(t, x) .
$$

These models factorise into their chiral parts as above.

An interesting question is: For which level $k$ exist current algebras as given above? If one takes the direct sum of representations as above, ie by matrices $M_{1}^{a} \oplus M_{2}^{a}$, it is trivial to check that the level of the corresponding current algebra is the sum of the levels determined by the $M_{1}^{a}$ and $M_{2}^{a}$, respectively. Therefore, we have primarily to look for level 1 representations.

As a well known fact, there are representations of DyNKIN index 1 by Hermitian matrices for the compact real LIE algebras of type $A_{n}$, the LIE algebras of the special unitary groups $S U(n+1)$, and of type $C_{n}$, the LIE algebras of the symplectic groups $S p(n)$. Orthogonal representations of DYNKIN index 2 exist for $B_{n}(S O(2 n+1)), D_{n}(S O(2 n))$ and the exceptional LIE algebra $G_{2}$ (for both facts see eg $\left[\right.$ Fuc92, (1.6.78)]). Therefore, for all but the colour algebras $E_{6-8}, F_{4}$ there are quark models with level 1 and hence at all levels 1 .

There are obvious adaptations of quark models to abelian, semi-simple and reductive colour algebras. As reductive LIE algebras decompose into a direct sum of simple ideals and a $d$-dimensional ideal isomorphic to $\mathbb{R}^{d}$, we take this as the general case. Assume now that $\mathfrak{g}$ is a reductive LIE algebra with the decomposition $\mathfrak{g}=\bigoplus_{\alpha} \mathfrak{g}_{\alpha} \oplus \mathbb{R}^{d}$, where the $\mathfrak{g}_{\alpha}$ stand for simple ideals. For the semi-simple LIE algebra $\bigoplus_{\alpha} \mathfrak{g}_{\alpha}$ we simply take direct sums of the current algebras above.

The abelian current algebra, ie the current algebra associated with an abelian colour algebra of dimension $d$, is constructed by taking $d$ independent, complex

\footnotetext{
${ }^{1}$ For the remaining four simple colour algebras, $E_{6-8}, F_{4}$, there are quark models for some levels (see eg [MP81]), but not for level 1. Yet, the corresponding chiral conformal nets are available at any level by different means (see section 2.3 ).
} 
quarks and setting with respect to some basis $\left\{T^{k}\right\}_{k=1, \ldots, d}$ of $\mathbb{R}^{d}$ :

$$
j^{k \mu}(t, x):=: \overline{\psi^{k}} \gamma^{\mu} \psi^{k}:(t, x) .
$$

These currents decompose into independent, chiral components as above and their commutation relations are given in terms of these chiral components as:

$$
\left[j_{ \pm}^{k}\left(x_{ \pm}\right), j_{ \pm}^{l}\left(y_{ \pm}\right)\right]=\frac{i}{2 \pi} \delta^{k l} \delta^{\prime}\left(x_{ \pm}-y_{ \pm}\right) .
$$

Simply by changing the basis of $\mathbb{R}^{d}$, one may replace in (III.8) the KroNECKER symbol $\delta^{k l}$ by any other Euclidean scalar product on $\mathbb{R}^{d}$. To capture the general commutation relation of an abelian current algebra, we take an arbitrary Euclidean scalar product, $g_{\mathbb{R}^{d}}$, and admit an additional multiplicative constant, $\kappa$ :

$$
\left[j_{ \pm}^{k^{\prime}}\left(x_{ \pm}\right), j_{ \pm}^{l^{\prime}}\left(y_{ \pm}\right)\right]=\frac{i}{2 \pi} \kappa g_{\mathbb{R}^{d}}^{k^{\prime} l^{\prime}} \delta^{\prime}\left(x_{ \pm}-y_{ \pm}\right)
$$

Because of the indicated arbitrariness, $\kappa$ should not be viewed as a "level" like for current algebras associated with simple colour algebras. The general formulation of abelian current algebras is the one needed for current subalgebras in current algebras of simple colours, where $\kappa$ and $g_{\mathbb{R}^{d}}^{k l}$ are given by the embedding. The current algebra for $d=1$ will be called the $U(1)$-current algebra.

\subsection{SUGAWARA construction for chiral current algebras}

For a general quantum field theory it is not clear, whether and how the coordinate and gauge transformations are generated by local quantum fields which are intrinsic to the theory and may be viewed as densities of energy, momentum, or charges. While in classical Lagrangean field theory the explicitly known NOETHER currents and their integrals over space yield such objects of a local nature, an analogous result for a general quantum field theory in Wightman's framework is not known.

In 1+1-dimensional conformal quantum field theory the situation is different: under weak assumptions, LÜSCHER AND MACK found that conformal stressenergy tensors always decompose into independent chiral parts and that these yield a local formulation of the VIRASORO algebra, ie an infinitesimal, projective representation of the group of orientation preserving diffeomorphisms of the circle, Diff $\left(\mathrm{S}^{1}\right)$. Such stress-energy tensors serve well as densities for the conformal transformations and their specific properties allow to apply powerful tools connected to structure and representation theory of the VIRASORO algebra and of $\operatorname{Diff}_{+}\left(\mathrm{S}^{1}\right)$.

It is a remarkable feature of current algebras that we have a simple method for constructing their stress-energy tensor, the Sugawara construction. We come back to the general result, the theorem of Lüscher and Mack, after we have sketched the SugaWARA construction and hence given a non-trivial example. 
The Sugawara construction applies independently to both chiral parts of a 1+1-dimensional current algebra. It was introduced by SugAwARA [Sug68] for currents in 3+1-dimensional MiNkOWski space; for an account on the history of the SugaWARA construction in the context of 1+1-dimensional and chiral current algebras see eg [HKOC96]. We will deal with chiral currents only and we drop the suffices,+- .

The discussion for a chiral current algebra associated with a simple colour algebra, $\mathfrak{g}$, covers the general case of reductive colour algebras with straightforward modifications, so we deal with the former in detail. The normal ordering of currents will be used:

$$
\begin{aligned}
& : j^{a} j^{b}:(x) \\
= & \lim _{x^{\prime} \rightarrow x}\left(j^{a}(x) j^{b}\left(x^{\prime}\right)-\frac{\Delta^{2}\left(x-x^{\prime}\right)}{(2 \pi)^{2}} k g_{\mathfrak{g}}^{a b}-i f^{a b}{ }_{c} j^{c}(x) \frac{\Delta\left(x-x^{\prime}\right)}{2 \pi}\right) .
\end{aligned}
$$

We define the Sugawara stress-energy tensor, $\Theta^{\mathfrak{g}}$, of the current algebra of colours in $\mathfrak{g}$ at level $k$ by:

$$
\Theta^{\mathfrak{g}}(x):=\frac{\pi}{k+\mathrm{g}_{\mathfrak{g}}^{\vee}} g_{a b}^{\mathfrak{g}}: j^{a} j^{b}:(x)
$$

The symbol $\mathrm{g}_{\mathfrak{g}}^{\vee}$ stands for the dual Coxeter number which amounts to half of the second Casimir operator, $C_{2}^{\mathfrak{g}}:=g_{a b}^{\mathfrak{g}} T^{a} T^{b}$, in the adjoint representation of $\mathfrak{g}$.

From the following commutation relation of $\Theta^{\mathfrak{g}}$ with the currents it can be readily deduced that integrals of $\Theta^{\mathfrak{g}}$ indeed generate the conformal transformations on the currents:

$$
\left[\Theta^{\mathfrak{g}}(x), j^{c}(y)\right]=i j^{c}(x) \delta^{\prime}(x-y) .
$$

The commutation relation of $\Theta^{\mathfrak{g}}$ with itself reads:

$$
\begin{aligned}
{\left[\Theta^{\mathfrak{g}}(x), \Theta^{\mathfrak{g}}(y)\right]=} & i 2 \Theta^{\mathfrak{g}}(x) \delta^{\prime}(x-y)-i\left(\frac{d}{d y} \Theta^{\mathfrak{g}}(y)\right) \delta(x-y) \\
& -i \frac{c_{\mathfrak{g}}(k)}{24 \pi} \delta^{\prime \prime \prime}(x-y)
\end{aligned}
$$

The number $c_{\mathfrak{g}}(k)$, which determines the central extension of this algebra, is called central charge and has for the SUGAWARA stress-energy tensor the following value:

$$
c_{\mathfrak{g}}(k)=\frac{k d_{\mathfrak{g}}}{k+\mathrm{g}_{\mathfrak{g}}^{\vee}} .
$$

It is not difficult to show that $c_{\mathfrak{g}}(k)$ lies between the rank of $\mathfrak{g}, r_{\mathfrak{g}}$, and its dimension, $d_{\mathfrak{g}}$.

The SugaWARA stress-energy tensor for the current algebras of semi-simple colour algebras, $\bigoplus_{\alpha} \mathfrak{g}_{\alpha}$, is simply given by $\Theta^{\oplus_{\alpha}}=\sum_{\alpha} \Theta^{\mathfrak{g}_{\alpha}}$. For a current algebra 
associated with an abelian colour algebra of dimension $d$, metric tensor $g_{\mathbb{R}^{d}}^{k l}$ and "level" $\kappa$ as discussed above we set (normal ordering as in (III.10) with obvious alterations):

$$
\Theta^{\mathbb{R}^{d}}(x):=\frac{\pi}{\kappa} g_{k l}^{\mathbb{R}^{d}}: j^{k} j^{l}:(x) .
$$

This stress-energy tensor has central charge $c_{\mathbb{R}^{d}}=d$. Concerning a current algebra of a reductive colour algebra one has to add this contribution for the abelian ideal.

The theorem of LÜSCHER AND MACK determines the commutation relations of a chiral stress-energy tensor of a conformally invariant theory to be of the form (III.13) where the central charge is the only free parameter [FST89, Mac88, LM76]. The prerequisites are the following: It is assumed that the stress-energy tensor $\Theta^{\mu \nu}$ is a symmetric-tensor $\left(\Theta^{\mu \nu}=\Theta^{\nu \mu}\right)$, Hermitian $\left(\Theta^{\mu \nu \dagger}=\Theta^{\mu \nu}\right)$, conserved $\left(\partial_{\mu} \Theta^{\mu \nu}=0\right)$ local Wightman field, which is relatively local to all the fields of a Wightman theory and which generates the translations as $P^{\mu}=\int d x^{1} \Theta^{\mu 0}$. The Wightman theory itself shall be scale invariant, ie there shall be a unitary implementation $V(D(t))$ of the scale transformations $x^{\mu} \mapsto e^{t} x^{\mu}$ which leaves the vacuum invariant. All translationally invariant vectors are assumed to be scalar multiples of the vacuum, $\Omega$. $\Theta$ is required to have scale dimension 2 , ie we have $V(D(t)) \Theta^{\mu \nu}(t, x) V(D(t))^{*}=e^{2 t} \Theta^{\mu \nu}\left(e^{t} t, e^{t} x\right)$.

Then $\Theta$ is traceless $\left(\Theta_{\mu}{ }^{\mu}=0\right)$, takes in light-cone coordinates the form $\Theta\left(x_{+}, x_{-}\right)=\operatorname{diag}\left(\Theta_{++}\left(x_{+}\right), \Theta_{--}\left(x_{-}\right)\right)$, ie $\Theta$ decomposes into its independent chiral parts $\Theta_{++}$and $\Theta_{--}$and its chiral components obey the commutation relation (III.13) above with some central charges $c_{ \pm}$. The two-point function of the chiral components is given by:

$$
\left\langle\Omega, \Theta_{ \pm \pm}\left(x_{ \pm}\right) \Theta_{ \pm \pm}\left(y_{ \pm}\right) \Omega\right\rangle=\frac{c_{ \pm}}{8 \pi^{2}} \Delta\left(x_{ \pm}-y_{ \pm}\right)^{4}
$$

Hence, we have $c_{ \pm} \geq 0$ and $\Theta_{ \pm}$vanishes if and only if $c_{ \pm}$vanishes ${ }^{2}$. If parity is conserved, then both central charges coincide.

The assumptions of the LÜSCHER MACK theorem are comparatively weak and natural in the setting of conformal quantum field theory in $1+1$ dimensions; they should be fulfilled in a large class of models. The most remarkable outcome of the theorem is the determination of the commutation relations (III.13) which, on a "decent" HILBERT space, exponentiate to a projective representation of Diff $_{+}\left(\mathrm{S}^{1}\right)^{\sim}$, the universal enveloping group of Diff $\left(\mathrm{S}^{1}\right)$ [GW85, TL99]. This does not mean, however, that the theory itself has a diffeomorphism symmetry.

The Sugawara stress-energy tensor fulfills all the properties of a stressenergy tensor in the sense of LÜSCHER AND MACK with respect to the currents. In addition, it is relatively local to the quarks by its very definition and the quark

\footnotetext{
${ }^{2}$ Follows from the REEH-SCHLIEDER theorem [RS61]; alternatively, one may apply [Jos65, lemma 2, section V.3.B]. For the Virasoro algebra there are other proofs [GW85, lemma 1.1], [Gom86], too.
} 
model construction. In most cases, however, the SuGAWARA stress-energy tensor does not implement the conformal transformations on the quarks and differs from the stress-energy tensor which may be constructed from the quarks directly.

Of course, the Sugawara construction yields stress-energy tensors for rational $c \geq 1$ only. BuchHolz And Schulz-Mirbach [BSM90] have given a construction for all $c>1$. As results of FrIEDAN, QIU AND SHEnker [FQS84, FQS85a, FQS85b] (cf [Lan88]) show, all values of $c$ below 1 which are compatible with unitarity and positivity of energy lie in a discrete series: $c(m)=1-6 /(m+2)(m+3), m \in \mathbb{N}$. The stress-energy tensors having these central charges were obtained by Goddard, Kent And Olive (GKO) [GKO85, GKO86] by a CoseT construction, see section $\mathrm{V}, 3.1$.

\section{Chiral conformal nets of current algebras}

This section establishes the connection between chiral current algebras and chiral conformal nets. As a first step we need to formulate the conformal extension of the current algebras on the conformal covering of the light-ray. From there we come to the description of chiral conformal fields in terms of non-local operators, their modes. The mode picture is particularly suitable for controlling the action of the unbounded current operators on vectors in the WIGHTMAN domain and enables us to show how current algebras generate chiral conformal nets. It identifies chiral current algebras as highest-weight representations of affine KAC-MoODY algebras as well. This connection makes it possible to establish chiral current algebras on abstract grounds, ie beyond the quark model construction.

\subsection{Compact picture of chiral current algebras}

Fields which are defined for test functions on the light-ray will be denoted with a hat, eg $\widehat{\Phi}$, such that we can clearly distinguish between fields on the light-ray and on its conformal compactification. The natural definition of conformal fields extended to $\mathrm{S}^{1}$ referring to the CAYLEY transformation (II.2) is given for an arbitrary chiral, quasi-primary field $\widehat{\Phi}$ of scaling dimension $h \in \mathbb{N}$ as follows:

$$
\widetilde{\Phi}(z)=N_{\Phi}\left(i \frac{d x}{d z}\right)^{h} \widehat{\Phi}(x(z)) .
$$

The normalisation constant $N_{\Phi}$ is fixed such that the commutation relations take a simple form; we use $N_{\Phi}=2 \pi$ in the following. 
The operator-valued distributions $\widetilde{\Phi}$ are defined with respect to the integration measure $\oint d z / 2 \pi i$ on $\mathrm{S}^{1}$. We write $f_{\wedge}$ for a test function on the light-cone:

$$
\begin{aligned}
\widetilde{\Phi}\left(\widetilde{f}_{\wedge}\right) & :=\int_{\mathbb{R}} d t \widehat{\Phi}(t) f_{\wedge}(t) \\
& =\oint \frac{d z}{2 \pi i} \widetilde{\Phi}(z) \underbrace{(1+z)^{2(h-1)} 2^{-(h-1)} f_{\wedge}(t(z))}_{=: \widetilde{f}_{\wedge}(z)} .
\end{aligned}
$$

The fields $\widehat{\Phi}$ are declared on smooth test functions on $\mathbb{R}$ with compact support. Conformal symmetry permits to extend the fields $\widetilde{\Phi}$ from the space of test functions $\widetilde{f}_{\wedge}$ to all $f_{\sim} \in C^{\infty}\left(\mathrm{S}^{1}\right)$, which in turn may be used to define extensions of the fields $\widehat{\Phi}$ on all test functions $\widehat{f_{\sim}} ;$ the introduction of the compact picture is not necessary for extending the fields, of course, but it is convenient:

$$
\begin{aligned}
\widehat{\Phi}\left(\widehat{f_{\sim}}\right) & :=\oint \frac{d z}{2 \pi i} \widetilde{\Phi}(z) f_{\sim}(z) \\
& =\int_{\mathbb{R}} d t \widehat{\Phi}(t) \underbrace{(1-i t)^{2(h-1)} 2^{-(h-1)} f_{\sim}(z(t))}_{=: \widehat{f \sim}(t)} .
\end{aligned}
$$

The change between the compact picture (fields on $\mathrm{S}^{1}$ ) and the light-cone picture (fields on $\mathbb{R}$ ) induces mutually inverse, linear transformations $f_{\sim} \mapsto \widehat{f_{\sim}}$ and $f_{\wedge} \mapsto$ $\widetilde{f}_{\wedge}$, which depend on the scaling dimension of the field under consideration.

The smooth functions on the circle form the test-function space of a conformally covariant chiral theory. Their images on the light-ray, $\hat{f}_{\sim}, f_{\sim} \in C^{\infty}\left(\mathrm{S}^{1}\right)$, are smooth functions which grow at most as $|x|^{2(h-1)}$ and have the same asymptotic behaviour for $x \rightarrow+\infty$ as for $x \rightarrow-\infty$. This allows us to integrate currents $(h=1)$ with constant test functions which yields the horizontal subalgebra. The stress-energy tensor $(h=2)$ may be integrated with test functions of growth up to $x^{2}$. This allows us to define the generators of global conformal symmetry as integrals of the stress-energy tensor; we come to this in section 3 .

For a Hermitian field on the light-ray we have: $\widehat{\Phi}\left(f_{\wedge}\right)^{\dagger}=\widehat{\Phi}\left(\overline{f_{\wedge}}\right)$. Hence, for real $f_{\wedge}$, the smeared field $\widehat{\Phi}\left(f_{\wedge}\right)$ is a Hermitian operator. The corresponding condition on a test function $f_{\sim}$ reads:

$$
\widetilde{\Phi}\left(f_{\sim}\right)^{\dagger}=\widetilde{\Phi}\left(f_{\sim}\right) \quad \Leftrightarrow \quad f_{\sim}=\widetilde{\widetilde{\widehat{f_{\sim}}}} .
$$

Now that we have given the rules for switching between the compact and the light-cone picture, it is straightforward to calculate the commutation relations in the compact picture from the relations between fields on the light-cone. For the currents we have (cf equation III.5):

$$
\left[\widetilde{j^{a}}\left(f_{\sim}\right), \widetilde{j^{b}}\left(g_{\sim}\right)\right]=i f^{a b}{ }_{c} \tilde{j}^{c}\left(f_{\sim} g_{\sim}\right)+k g_{\mathfrak{g}}^{a b} \oint \frac{d z}{2 \pi i} f_{\sim}^{\prime}(z) g_{\sim}(z) .
$$


$f_{\sim}^{\prime}$ stands for $d / d z f_{\sim}$.

The LÜSCHER-MACK algebra for a stress-energy tensor of central charge $c$ (cf equation III.13) reads:

$$
\left[\widetilde{\Theta}\left(f_{\sim}\right), \widetilde{\Theta}\left(g_{\sim}\right)\right]=\widetilde{\Theta}\left(f_{\sim}^{\prime} g_{\sim}-f_{\sim} g_{\sim}^{\prime}\right)+\frac{c}{12} \oint \frac{d z}{2 \pi i} f_{\sim}^{\prime \prime \prime}(z) g_{\sim}(z)
$$

The action of the SugAwARA stress-energy tensor on the currents takes the following form (cf equation III.12):

$$
\left[\widetilde{T}\left(f_{\sim}\right), \widetilde{j}^{a}\left(g_{\sim}\right)\right]=\widetilde{j}^{a}\left(-f_{\sim} g_{\sim}^{\prime}\right)
$$

All these relations are mere rewritings of those on the light-ray.

Test functions on the circle possess a FouRIER expansion: $f_{\sim}=\sum_{n \in \mathbb{Z}} f_{n} z^{-n}$. We write the test function defined by $z \mapsto z^{m}$ by $\left[z^{m}\right]$ and define for a quasiprimary chiral field $\widetilde{\Phi}$ of scaling dimension $h$ its modes as:

$$
\Phi_{n}:=\widetilde{\Phi}\left(\left[z^{h-1+n}\right]\right), n \in \mathbb{Z}
$$

If $\widehat{\Phi}$ is Hermitian, we have for the modes $\Phi_{n}^{\dagger}=\Phi_{-n}$. The modes of currents and stress-energy tensors are given by:

$$
L_{n}:=\widetilde{\Theta}\left(\left[z^{n+1}\right]\right), \quad j_{n}^{a}:=\widetilde{j^{a}}\left(\left[z^{n}\right]\right) .
$$

It is a matter of simple arithmetics to calculate the commutation relations in terms of the modes:

$$
\begin{aligned}
{\left[j_{m}^{a}, j_{n}^{b}\right] } & =i f^{a b}{ }_{c} j_{m+n}^{c}+k g_{\mathfrak{g}}^{a b} m \delta_{m,-n} \\
{\left[L_{m}, L_{n}\right] } & =(m-n) L_{m+n}+\frac{c}{12} \delta_{-n, m} m\left(m^{2}-1\right) \\
{\left[L_{m}, j_{n}^{a}\right] } & =-n j_{m+n}^{a} .
\end{aligned}
$$

The commutation relations (III.27) are known as Virasoro algebra at central charge $c$. The commutation relations (III.26) are manifestations of the relations in an affine Kac-Moody algebra or affine Lie algebra (see eg [Kac90], [Fuc92]).

The relations (III.28) and (III.27) show that the zeroth mode of $\Theta$, the conformal Hamiltonian $L_{0}$, defines a natural energy grading on the modes and hence on the vacuum HILBERT spaces of both current algebras and stress-energy tensors. Since the spectrum of $L_{0}$ is positive and pure-point on these spaces, it is a positive, essentially self-adjoint operator. Moreover, the eigenspaces are finite dimensional. These facts are founding elements of the next section.

\section{$2.2 \quad$ Integrating chiral current algebras}

In spite of their strong conceptual and structural similarities, the relation between the formulations of quantum field theory in terms of tempered operator-valued 
distributions (WIGHTMAN's axioms) and in terms of local algebras of bounded operators (HAAG-KASTLER axioms) is not that of a straightforward equivalence. We shall not elaborate on the remarkable general achievements in establishing relations in both directions (see eg [Fre91] for a short summary and a list of references). In the following we discuss this relation for current algebras. The technical problems present in general surface here as well, but in a mild and manageable form.

As we will see in a moment, the energy grading with respect of $L_{0}$, the $\mathrm{Su}$ GAWARA construction and the LIE algebra of the modes allow us to establish the following properties for the smeared currents: For real test functions ${ }^{3} f_{\sim}, g_{\sim}$ the current operators $j^{a}\left(f_{\sim}\right), j^{b}\left(g_{\sim}\right)$ are essentially self-adjoint on the WiGHTMAN domain and all bounded functions of their self-adjoint closures commute, ie their closures $j^{a}\left(f_{\sim}\right)^{-}, j^{b}\left(g_{\sim}\right)^{-}$commute in the sense of self-adjoint operators [RS72, VIII.5], if $f_{\sim}, g_{\sim}$ have disjoint supports. Both properties are not obvious due to the unbounded character of the smeared fields (cf eg [RS72, VIII.5], [Ree88]), but when they hold one may define the local algebras of the chiral net generated by the current algebra as:

$$
\mathcal{B}(I):=\left\{\tilde{j}^{a}\left(f_{\sim}\right)^{-}, \operatorname{supp}\left(f_{\sim}\right) \subset I, f_{\sim}=\overline{f_{\sim}}, T^{a} \in \mathfrak{g}\right\}^{\prime \prime}, I \Subset S^{1}
$$

Covariance and isotony are direct consequences of this definition and the properties of the current algebras as Wightman theories. The cyclicity of the vacuum may be established by a simple argument based on the spectral resolution of the closures of the smeared fields [DF77, theorem 4.1]. In general, WiGHTMAN fields do not behave that well and one has to introduce more refined definitions for the local algebras (cf [DSW86]).

BuchHolz AND SCHulz-Mirbach [BSM90] introduced a method for establishing essential self-adjointness and locality for the closures of symmetric smeared fields based on proving linear energy bounds with respect to the conformal Hamiltonian. This approach is particularly well-suited for chiral conformal models for which the commutation relations are explicitly known in terms of their modes.

In [BSM90], the method is applied to the VIRASORO algebras and this scheme covers, as stated by BuCHHOLz AND SCHulz-MiRBACH, the $U(1)$-current algebra as well. Hence it is known already that both Wightman fields generate chiral conformal nets. The argument on the $U(1)$-current algebra is extended to another class of conformally covariant fields in chapter VI. If one is interested in current algebras with simple colour algebras, one needs to apply another argument, which uses the connection between $L_{0}$ and the current algebra via the SugAWARA construction, because the zero modes of in these algebras are

\footnotetext{
${ }^{3}$ Currents have scaling dimension 1 which leads to $\widetilde{\widetilde{\widehat{f_{\sim}}}}=\overline{f_{\sim}}, f_{\sim} \in C^{\infty}\left(\mathrm{S}^{1}\right)$; see (III.19), (III.18). Hence, real test functions $f_{\sim}$ lead to symmetric smeared current operators, see equation (III.20).
} 
not scalars. Since the $U(1)$-current algebra possesses a SUGAWARA stress-energy tensor as well, the arguments below apply to this case as well.

To the author's knowledge, a proof of the following result is not yet available:

Theorem III.1: Assume $\mathfrak{g}$ is a reductive colour algebra for which the current algebra is available as a chiral conformal WIGHTMAN theory on the conformally compactified light-ray, $\mathrm{S}^{1}$. Then equation (III.29) defines a chiral conformal theory.

Proof: We establish linear energy bounds for the currents $\widetilde{j^{a}}$ of a simple colour algebra $\mathfrak{g}_{\alpha} \subset \mathfrak{g}$; the argument relies on the SuGAWARA construction, the energy grading on HILBERT space and positivity of energy.

We choose a basis $\left\{T^{a}\right\}$ in colour space which diagonalises the KILLING metric on $\mathfrak{g}_{\alpha}$ such that: $2 \mathrm{~g}_{\mathfrak{g}_{\alpha}}^{\vee}\left\langle T^{a}, T^{b}\right\rangle_{\mathfrak{g}_{\alpha}}=\delta^{a b}$. Then the conformal Hamiltonian $L_{0}^{\mathfrak{g}_{\alpha}}$ of colours in $\mathfrak{g}_{\alpha}$ reads:

$$
L_{0}^{\mathfrak{g}_{\alpha}}=\frac{\mathrm{g}_{\mathfrak{g}_{\alpha}}^{\vee}}{k_{\mathfrak{g}_{\alpha}}+\mathrm{g}_{\mathfrak{g}_{\alpha}}^{\vee}}\left(\sum_{i=1}^{d_{\mathfrak{g}_{\alpha}}} j_{0}^{a} j_{0}^{a}+2 \sum_{i=1}^{d_{\mathfrak{g}_{\alpha}}} \sum_{n>0} j_{-n}^{a} j_{n}^{a}\right)
$$

This positive operator is dominated by the conformal Hamiltonian of the whole current algebra, $L_{0}^{\mathfrak{g}}$.

We take a vector $\phi_{N}$ of energy $N$ with respect to $L_{0}^{\mathfrak{g}}$ and of unit length. There is the following bound for the action of the annihilation modes $j_{n}^{a}, n \geq 1$, on $\phi_{N}$ (using (III.30)) :

$$
\left\|j_{n}^{a} \phi_{N}\right\|^{2} \leqslant \frac{k_{\mathfrak{g}_{\alpha}}+\mathrm{g}_{\mathfrak{g}_{\alpha}}^{\vee}}{2 \mathrm{~g}_{\mathfrak{g}_{\alpha}}^{\vee}}\left\langle\phi_{N}, L_{0}^{\mathfrak{g}_{\alpha}} \phi_{N}\right\rangle \leqslant \frac{k_{\mathfrak{g}_{\alpha}}+\mathrm{g}_{\mathfrak{g}_{\alpha}}^{\vee}}{2 \mathrm{~g}_{\mathfrak{g}_{\alpha}}^{\vee}} N
$$

(III.30) yields a similar bound for the zero modes $j_{0}^{a}$, and for the generating modes $j_{-n}^{a}, n \geq 1$, we use the bound (III.31) and the commutation relations (III.26). Thus, we have for all $n \in \mathbb{Z}$ :

$$
\left\|j_{n}^{a} \phi_{N}\right\|^{2} \leqslant \frac{k_{\mathfrak{g}_{\alpha}}+\mathrm{g}_{\mathfrak{g}_{\alpha}}^{\vee}}{\mathrm{g}_{\mathfrak{g}_{\alpha}}^{\vee}} N+k_{\mathfrak{g}_{\alpha}} 2 \mathrm{~g}_{\mathfrak{g}_{\alpha}}^{\vee}|n| \leqslant d_{\mathfrak{g}_{\alpha}} k_{\mathfrak{g}_{\alpha}} N+k_{\mathfrak{g}_{\alpha}} 2 \mathrm{~g}_{\mathfrak{g}_{\alpha}}^{\vee}|n|
$$

The second inequality follows from $c_{\mathfrak{g}_{\alpha}}=d_{\mathfrak{g}_{\alpha}} k_{\mathfrak{g}_{\alpha}} /\left(k_{\mathfrak{g}_{\alpha}}+\mathrm{g}_{\mathfrak{g}_{\alpha}}^{\vee}\right) \geq r_{\mathfrak{g}_{\alpha}} \geq 1$ and $\mathrm{g}_{\mathfrak{g}_{\alpha}}^{\vee} \geq 1$. We note that the right-hand side is greater than 1 for $N \neq 0$; the case $N=0$, ie $\phi_{0} \sim \Omega$, does not pose any problem in the following.

In order to obtain a bound for a general $\phi$ from the Wightman domain, we proceed as follows: The symmetric operator $j_{-n}^{a} j_{n}^{a}$ leaves invariant the finitedimensional energy eigenspace of energy $N$ and we may choose an orthonormal basis of vectors $\phi_{N}^{\iota}$ diagonalising the action of $j_{-n}^{a} j_{n}^{a}$. Expanding $\phi$ in terms of 
this basis yields:

$$
\begin{aligned}
\left\|j_{n}^{a} \phi\right\|^{2} & \leqslant \sum_{N, \iota} \overline{c_{N, \iota}} c_{N, \iota}\left\|\left(d_{\mathfrak{g}_{\alpha}} k_{\mathfrak{g}_{\alpha}} N+k_{\mathfrak{g}_{\alpha}} 2 \mathrm{~g}_{\mathfrak{g}_{\alpha}}^{\vee}|n|\right) \phi_{N}^{\iota}\right\|^{2} \\
& =\left\|\left(d_{\mathfrak{g}_{\alpha}} k_{\mathfrak{g}_{\alpha}} L_{0}^{\mathfrak{g}}+k_{\mathfrak{g}_{\alpha}} 2 \mathrm{~g}_{\mathfrak{g}_{\alpha}}^{\vee}|n|\right) \phi\right\|^{2} .
\end{aligned}
$$

This argument gives a linear energy bound for the action of the modes on the whole Wightman domain.

Our interest is in bounds for smeared currents. If we take a test function $f_{\sim} \in C^{\infty}\left(\mathrm{S}^{1}\right)$, then the FouRIER coefficients decrease fast enough to make any series $\sum_{n}\left|f_{n}\right||n|^{l}, l \in \mathbb{N}$, converge. Hence one gets for an arbitrary smeared current $j^{a}\left(f_{\sim}\right)$ and an arbitrary vector $\phi$ from the Wightman domain:

$$
\left\|j^{a}\left(f_{\sim}\right) \phi\right\| \leqslant c_{f_{\sim}}\left\|\left(L_{0}+\mathbb{1}\right) \phi\right\| .
$$

The constant $c_{f \sim}$ depends on $f_{\sim}$ only (and not on $\phi$ ). In fact, one may choose: $c_{f_{\sim}}=\sum_{n \in \mathbb{Z}}\left|f_{n}\right| k_{\mathfrak{g}_{\alpha}}\left(d_{\mathfrak{g}_{\alpha}}+2 \mathrm{~g}_{\mathfrak{g}_{\alpha}}^{\vee}|n|\right)$. By applying the arguments above for all simple ideals of $\mathfrak{g}$ and (in a simplified version) for the abelian ideal, we get linear energy bounds for all smeared currents.

$L_{0}$ has a total set of eigenvectors and it is straightforward to check that $L_{0}$ is essentially self-adjoint on the Wightman domain. By NeLSON's analytic vector theorem [Nel59], $L_{0}$ has a dense set of analytic vectors $\phi$. A vector $\phi$ is analytic for an operator $A$ if for any $n=0,1, \ldots$ the $n$th power of $A$ is declared on $\phi$ and the following holds for some $0<s$ :

$$
\sum_{n=0}^{\infty} \frac{\left\|A^{n} \phi\right\|}{n !} s^{n}<\infty .
$$

The symmetric smeared fields $\tilde{j}^{a}\left(f_{\sim}\right)$ may be extended to the space of finite energy vectors, the domain $D\left(L_{0}{ }^{-}\right)$of the closure of $L_{0}$, because of the bound (III.34). Any analytic vector of $L_{0}{ }^{-}+\mathbb{1}$ is an analytic vector for the extended smeared currents. Again by NELSON's theorem, the extended smeared currents are essentially self-adjoint and hence the WIGHTMAN domain is a core for the self-adjoint closures $\widetilde{j^{a}}\left(f_{\sim}\right)^{-}$.

As indicated by BuchHolz and Schulz-Mirbach [BSM90], finally, we make contact with a result of DRIESSLER AND FRÖHLICH [DF77], which requires to establish linear energy bounds on the smeared fields $\widetilde{j^{a}}\left(f_{\sim}\right)$, for $A d_{L_{0}}\left(\widetilde{j^{a}}\left(f_{\sim}\right)\right)$ and for $A d_{L_{0}}^{2}\left(\widetilde{j^{a}}\left(f_{\sim}\right)\right)$ as quadratic forms on the Wightman domain. Because we argued for arbitrary test functions, we need not worry about the action of $A d_{L_{0}}$ and the bounds for the quadratic forms follow from (III.34) by [RS75, theorem X.18]. The result of DRIESSLER AND FrÖHLICH now says: if smeared currents commute on the core, then all spectral projections of their self-adjoint closures commute. This ensures locality for the algebras defined above. 
Cyclicity of the vacuum follows by the argument in [DF77, theorem 4.1]. Since the other properties of definition II.1 follow directly, we completed the proof..

The argument above works for test functions with support in some $I \Subset \mathrm{S}^{1}$ as well as for test functions supported in all of $S^{1}$. For test functions of support in some $I \Subset \mathrm{S}^{1}$ one could have proceeded along the lines of BorCHERS AND ZIMMERMANN [BZ63] by recognising that the linear energy bounds prove the vacuum to be an analytic vector for all currents.

The results of [BSM90] on the VIRASORO algebra show that the SugAwARA stress-energy tensor generates a local and relatively local subnet of the chiral theory generated by the currents. The energy bounds for the modes $L_{0}, L_{ \pm 1}$ of such a tensor ensure that their symmetric linear combinations generate a unitary representation of $\operatorname{PSL}(2, \mathbb{R})^{\sim}$ [Frö77].

\subsection{From loop algebras to chiral current algebras}

Both chiral current algebras and chiral stress-energy tensors carry the structures of infinite-dimensional LIE algebras. The chiral current algebra of colours in a simple, compact $\mathfrak{g}$ represents a central extension of $l \mathfrak{g} \equiv C^{\infty}\left(\mathrm{S}^{1}, \mathfrak{g}\right)$, the loop algebra of smooth mappings from $\mathrm{S}^{1}$ into $\mathfrak{g}$. The commutation relations of stressenergy tensors represent central extensions of the LIE algebra of smooth vector fields on the circle, $\operatorname{Vect}\left(\mathrm{S}^{1}\right)$, known as Witt algebra (see eg [Sch95]). lg is the LIE algebra of the loop group $\mathcal{L} G \equiv C^{\infty}\left(\mathrm{S}^{1}, G\right)$ of smooth mappings from $\mathrm{S}^{1}$ into $G$, the compact, connected and simply connected LIE group having $\mathfrak{g}$ as its LIE algebra. And $\operatorname{Vect}\left(\mathrm{S}^{1}\right)$ is the LIE algebra of the group of orientation preserving diffeomorphisms on the circle, Diff $\left(\mathrm{S}^{1}\right)$.

Multiplication in $\mathcal{L} G$ is declared point wise: $g_{1} \cdot g_{2}(z)=g_{1}(z) \cdot g_{2}(z), z \in \mathrm{S}^{1}$, and in $\operatorname{Diff}_{+}\left(\mathrm{S}^{1}\right)$ by successive evaluation: $\varphi_{1} \cdot \varphi_{2}(z)=\varphi_{1}\left(\varphi_{2}(z)\right)$. Diff $\left(\mathrm{S}^{1}\right)$ acts on $\mathcal{L} G$ through reparametrisations of $S^{1}$. The group multiplication law in the semi-direct product induced by this action, $\mathcal{L} G \rtimes \operatorname{Diff}_{+}\left(\mathrm{S}^{1}\right)$, reads:

$$
\left(g_{1}, \varphi_{1}\right) \cdot\left(g_{2}, \varphi_{2}\right)=\left(g_{1} \circ \varphi_{2} \cdot g_{2}, \varphi_{1} \cdot \varphi_{2}\right)
$$

General references on these structures are: [Mil84], [PS86].

These relations may be used to establish current algebras for all simple colour algebras and at all positive integer levels as chiral conformal theories both in accordance with Wightman's axioms and the HAAG-KASTLER axioms. The starting point are unitarisable, highest-weight representations of affine KAC-MoODY algebras with the vacuum, $\Omega$, as highest-weight vector.

These representations are given in terms of modes $j_{n}^{a}, a=1, \ldots, d_{\mathfrak{g}}, n \in \mathbb{Z}$, and their complex linear combinations. One defines $j_{n}^{a} \Omega:=0, n \geq 0$. On the vector space of linear combinations of vectors of the form $j_{-n_{1}}^{a_{1}} \cdots j_{-n_{m}}^{a_{m}} \Omega$, $n_{1}, \ldots, n_{m}>0$, called $\mathscr{H}^{\text {fin }}$, there exists a scalar product such that the modes 
satisfy the commutation relations (III.26), are Hermitian $\left(j_{n}^{a \dagger}=j_{-n}^{a}\right.$ ), and we have and $\|\Omega\|=1$ [Kac90, theorem 11.7]. For this it is necessary and sufficient that the level $k$ is a positive integer.

The SugaWARA stress-energy tensor finds its counterpart in this context by the Segal-Sugawara formula:

$$
L_{n}:=\frac{1}{2\left(k+\mathrm{g}_{\mathfrak{g}}^{\vee}\right)} g_{a b}^{\mathfrak{g}} \sum_{n_{1}+n_{2}=n}: j_{n_{1}}^{a} j_{n_{2}}^{b}:
$$

Here, $g_{a b}^{\mathfrak{g}}: j_{k}^{a} j_{l}^{b}:=g_{a b}^{\mathfrak{g}} j_{k}^{a} j_{l}^{b}$ for $l>0$, and $g_{a b}^{\mathfrak{g}}: j_{k}^{a} j_{l}^{b}:=g_{a b}^{\mathfrak{g}} j_{l}^{b} j_{k}^{a}$ for $l \leq 0$. The $L_{n}$ satisfy the relations (III.27), (III.28) on $\mathscr{H}^{\text {fin }}$.

The unitarisable, highest-weight representations of affine KAC-MOODY algebras (cf eg [Kac90]) are known to exponentiate uniquely to projective, unitary representations of $\mathcal{L} G$ [GW84, TL99]. In $\mathcal{L} G$ a group element is localised in some $I \Subset \mathrm{S}^{1}$, if it coincides with the neutral element of the group outside $I$. This intrinsic locality structure makes it possible to prove, by group theoretical methods, that the exponentiated projective representations with the vacuum as highest weight define chiral conformal nets [TL97, Was98, GF93]. Since the level may identified in any locally normal represention within local algebras, it is a constituting element of the theory. We denote the local quantum theory connected with an exponentiated projective representation of $\mathcal{L} G$ at level $k$ by $\mathcal{L} G_{k}$, in marginal deviation from the common practice which leaves out " $\mathcal{L}$ ".

For Diff $\left(\mathrm{S}^{1}\right)$ one can follow the same program ${ }^{4}$, but Wightman's axioms are established for stress-energy tensors of all central charges $c$ (cf section 1.2), hence these models were established as chiral nets in [BSM90] already. The chiral net generated by a stress-energy tensor with central charge $c$ will be called the Virasoro model $\mathcal{V} i r_{c}$.

Intermediate results which were obtained in order to establish the $\mathcal{L} G_{k}$ models can be used to show that for every simple colour algebra $\mathfrak{g}$ the action of the modes on $\mathscr{H}^{\text {fin }}$ extends to a Wightman theory of chiral currents. This covers in particular the four cases $E_{6-8}, F_{4}$ for which there are no quark models at level 1 , and it will be applied in the context of current subalgebras (see section 3 ). The proof of the following proposition relies mainly on results of TOLEDANO-LAREDO [TL97, TL99], but the statement has not appeared explicitly, yet:

Proposition III.2: For every compact, simple colour algebra $\mathfrak{g}$ and at every positive, integer level $k$ the corresponding current algebra exists as a conformally covariant chiral quantum field theory in agreement with WIGHTMAN's axioms.

Proof: We start from the action of the Hermitian modes $j_{n}^{a}$ on the pre-HILBERT space $\mathscr{H}^{\text {fin }}$, which we introduced above. The closure of $\mathscr{H}^{\text {fin }}$, the HiLBerT space $\mathscr{H}$, is separable by construction.

\footnotetext{
${ }^{4}$ The relevant results are contained in [Kac90, theorem 11.12] (unitarity), [GW85, TL99] (integrability), [Lok94] (locality etc).
} 
One controls the action of the modes $j_{n}^{a}$ in terms of Sobolev norms:

$$
\|\xi\|_{s}:=\left\|\left(1+L_{0}\right)^{s} \xi\right\|, \quad \xi \in \mathscr{H}^{f i n}, s \in \mathbb{R}
$$

The right-hand side may be calculated by expansion into energy eigenvectors. The closure of $\mathscr{H}^{\text {fin }}$ with respect to the norm $\|\cdot\|_{s}$ is called the scale $\mathscr{H}_{s}$, and the space $\mathscr{H}^{\infty}=\bigcap_{s} \mathscr{H}_{s}$ is the space of smooth vectors for the action of the conformal Hamiltonian $L_{0}$. Since $L_{0}$ has a total set of eigenvectors, its closure is essentially self-adjoint on $\mathscr{H}^{\infty}$.

The commutation relations of the modes $j_{n}^{a}$ yield bounds for their action on $\mathscr{H}^{\text {fin }}$ in terms of the norms $\|.\|_{s}[$ GW84]. These allow to prove that this action on $\mathscr{H}^{\text {fin }}$ extends to a jointly continuous mapping $l \mathfrak{g} \times \mathscr{H}^{\infty} \rightarrow \mathscr{H}^{\infty}$ such that the commutation relations (III.21) are satisfied, ie we have a projective representation of $l \mathfrak{g}$ on $\mathscr{H}^{\infty}$ [TL97, corollary 1.3.1]. Hence, the currents $\tilde{j}^{a}$ are local.

The matrix elements of currents, namely $\left\langle v, \widetilde{j^{a}}\left(f_{\sim}\right) w\right\rangle, v, w \in \mathscr{H}^{\infty}$, define tempered distributions on $C^{\infty}\left(\mathrm{S}^{1}\right)$ by the definition of the topology on $l \mathfrak{g}$. The currents $\widetilde{j^{a}}\left(f_{\sim}\right)$ for real $f_{\sim}$ are essentially self-adjoint on $\mathscr{H}^{\infty}$, which means that all currents are closable operators [TL97, corollary 1.3.1].

$\mathscr{H}^{\infty}$ is the Wightman domain of the currents. It is clear that the vacuum is cyclic for the currents, since $\mathscr{H}=\mathscr{H}_{0}$. Arguments given in [BSM90] show that the Hermitian linear combinations of $L_{0, \pm 1}$ satisfy linear energy bounds with respect to $L_{0}$ and hence exponentiate to a positive-energy representation $U$ of $\operatorname{PSL}(2, \mathbb{R})[$ Frö77]. Obviously, $\mathbb{C} \Omega$ is the space of $U$-invariant vectors.

The action of the current modes and of the $L_{n}$ on $\mathscr{H}^{\text {fin }}$ exponentiates uniquely to a projective representation of $\mathcal{L} G \rtimes$ Diff $_{+}\left(\mathrm{S}^{1}\right)$ [GW84, TL99]. This shows that the currents are covariant with respect to $U$.

Irreducible, locally normal representations $\pi$ of $\mathcal{L} G_{k}$ on a HILBERT space $\mathscr{H}_{\pi}$ yield unitary, strongly continuous, projective representations of $\mathcal{L} G$. This can be seen looking at the one-parameter groups in $\mathcal{L} G$. Referring to a basis $\left\{T^{a}\right\}$ of $\mathfrak{g}$, a general element in $l \mathfrak{g}$ is given in terms of an $d_{\mathfrak{g}}$-tuple $f_{\sim}=\left(f_{\sim}^{(a)}\right)_{a}$ of smooth functions, and the one-parameter subgroups are of the form $g_{f_{\sim}}(t)=$ $\exp \left(-i t \sum_{a} f_{\sim}^{(a)} T^{a}\right)$. With respect to a partition of unity on $\mathrm{S}^{1}$ by two smooth, non-negative functions $\chi_{1,2}, \operatorname{supp}\left(\chi_{1,2}\right) \Subset \mathrm{S}^{1}$, we see that a locally normal representation $\mathcal{L} G_{k}$ defines a strongly continuous mapping from the one-parameter subgroups of $\mathcal{L} G$ to the unitaries on $\mathscr{H}_{\pi}$. In a neighbourhood of the identity the exponential map exp $: l \mathfrak{g} \rightarrow \mathcal{L} G$ is a homeomorphism, and thus we have 5 unitary, strongly continuous, projective representation of $\mathcal{L} G$.

The diffeomorphism symmetry of loop groups can be used to show that every locally normal representation is automatically covariant with respect to $P S L(2, \mathbb{R})$ (see section $A, 5$ ), but it is not clear a priori that the representation is of positive

\footnotetext{
${ }^{5}$ For an analogous argument on the $\mathcal{V} i r_{c}$ models one may use lemma A.5.
} 
energy. Likewise, there is no argument yet which ensures an action of $l \mathfrak{g}$ on a dense subspace of smooth vectors in representation space.

Therefore, it is not sure whether the locally normal representations are covered by the classification of unitarisable highest-weight representations of $l \mathfrak{g}$ at level $k$ in terms of the Weyl-alcove condition (eg [Fuc92, (2.4.26)]). These representations are completely determined by the action of the horizontal subalgebra on the lowest energy eigenspace, that is by the highest weight $\lambda$ of this irreducible, unitary representation of $\mathfrak{g}$. We discuss the WEYL-alcove condition for $l \mathfrak{s u}(n)$ in detail.

$\lambda$ is of the form $\lambda=\sum_{i=1}^{n-1} \lambda_{i} \lambda^{(i)}$, where the $\lambda^{(i)}$ are the fundamental weights of $\mathfrak{s u}(n)$ and the $\lambda_{i}$ are non-negative integers. The WEYL-alcove condition for $l \mathfrak{s u}(n)$ at level $k$ reads: $\sum_{i=1}^{n-1} \lambda_{i} \leq k$. We denote the set of highest weights $\lambda$ satisfying this condition by $P_{+}^{k}(S U(n))$. The corresponding (projective) highestweight representations of $l \mathfrak{g}$ are known to exponentiate to unitary, projective representations of $\mathcal{L} S U(n)$ with positive energy [GW84], which form locally normal representations of $\mathcal{L} S U(n)_{k}$ (covariant with positive energy) [Was98].

The fusion rules of these positive energy representations of $\mathcal{L} S U(n)_{k}$ are known to coincide with the ones expected from operator product expansions [Was98] which means that their statistical dimensions coincide with the asymptotic dimensions of the corresponding highest weights (eg [Fuc94, Fuc92]), which shall be denoted $d_{\lambda}, \lambda \in P_{+}^{k}(S U(n))$. XU has calculated the $\mu$-index [KLM01] of these models explicitly as [Xu00b, theorem 4.1]:

$$
\mu\left(\mathcal{L} S U(n)_{k}\right)=\sum_{\lambda \in P_{+}^{k}(S U(n))} d_{\lambda}^{2} .
$$

Because the $\mu$-index is finite and since the models are strongly additive [TL97] and split [GF93], the chiral nets $\mathcal{L} S U(n)_{k}$ are completely rational [KLM01], and by the value of the $\mu$-index the unitarisable, highest-weight representations are known to exhaust all sectors [KLM01, theorem 33].

This result opens the door to the classification of locally normal representations of many other chiral nets; in this work it will be needed only in the proof of proposition V.16 where it is shown that $\mathcal{L} E(8)_{1}$ has only one sector. The identification of all sectors of the $\mathcal{V} i r_{c<1}$ models [KL02] is another achievement of this sort, cf section $\mathrm{V} .3 .1$.

\section{Current subalgebras as instructive examples}

An injective LIE algebra homomorphism $\iota$ from a reductive colour algebra $\mathfrak{h}$ into a reductive colour algebra $\mathfrak{g}$, denoted $\iota: \mathfrak{h} \hookrightarrow \mathfrak{g}$, is regarded as an inclusion of LIE algebras $\iota(\mathfrak{h}) \subset \mathfrak{g}$. It clear from the current algebra (III.5) that the inclusion of colour algebras induces an inclusion of the corresponding current algebras. By theorem III.1, the inclusion of current algebras yields a chiral subnet, the current subalgebra, which we denote by $\mathcal{A} \subset \mathcal{B}$. 
The Sugawara construction endows the current subalgebra associated with $\iota(\mathfrak{h})$ with its own stress-energy tensor $\Theta^{\iota(\mathfrak{h})}$. Since $\Theta^{\iota(\mathfrak{h})}$ and $\Theta^{\mathfrak{g}}$ obey the same commutation relations with currents of colour in $\iota(\mathfrak{h})$, the tensor $\Theta^{\mathfrak{g}}-\Theta^{\iota(\mathfrak{h})}$ commutes with all these currents and hence with $\Theta^{\iota(\mathfrak{h})}$. It follows that $\Theta^{\mathfrak{g}}-\Theta^{\iota(\mathfrak{h})}$ satisfies the LÜSCHER-MACK commutation relations (III.13) with central charge $c_{\mathfrak{g}}-c_{\iota(\mathfrak{h})}$ and, therefore, it defines a stress-energy tensor. $\Theta^{\mathfrak{g}}-\Theta^{\iota(\mathfrak{h})}$ is called the Coset stress-energy tensor (associated with the respective current subalgebra).

It is clear from the arguments of BuchHolz AND Schulz-Mirbach [BSM90] that the COSET stress-energy tensor generates a chiral subnet of $\mathcal{B}$ which is contained in $\mathcal{C}_{\max }$. As arguments below show, the Hermitian linear combinations of modes $L_{0, \pm 1}^{\iota(\mathfrak{h})}$ of $\Theta^{\iota(\mathfrak{h})}$ generate the BorchERS-SugAwARA representation $U^{\mathcal{A}}$ of $\mathcal{A} \subset \mathcal{B}$ and the corresponding modes of the Coset stress-energy tensor generate $U^{\mathcal{A}^{\prime}}$, which coincides with the BorCHERS-SUGAWARA representation $U^{\mathcal{C}_{\text {max }}}$ of the maximal Coset model $\mathcal{C}_{\text {max }}$ associated with $\mathcal{A} \subset \mathcal{B}$ (cf section II.2).

If we have $\Theta^{\mathfrak{g}}=\Theta^{\iota(\mathfrak{h})}$, ie $c_{\Theta^{\mathfrak{g}}}=c_{\Theta^{\iota}(\mathfrak{h})}$, then the inclusion $\iota(\mathfrak{h})$ is called a conformal inclusion or conformal embedding. The following theorem III.3 shows that this notion of conformal inclusion coincides, for current subalgebras, with the one given in definition II.21.

We now establish that the Additional Assumption on $U^{\mathcal{A}}$ needed in chapter IV is satisfied for current subalgebras (cf page 65). The covering projection from $\operatorname{Diff}_{+}\left(\mathrm{S}^{1}\right)^{\sim}$ onto Diff $\left(\mathrm{S}^{1}\right)$ is denoted by p. The subgroup of diffeomorphisms $\varphi$ which are localised in some $I \Subset \mathrm{S}^{1}$, ie which satisfy $\varphi \uparrow I^{\prime}=i d \uparrow I^{\prime}$, is denoted as $\operatorname{Diff}_{I}\left(\mathrm{~S}^{1}\right)$. Localised diffeomorphisms $\varphi$ will be identified with their preimage $\mathrm{p}^{-1}(\varphi)$ in the first sheet of the covering:

Theorem III.3: Let the chiral subnet $\mathcal{A} \subset \mathcal{B}$ stem from an embedding of chiral current algebras and let $\mathcal{C}_{\max }$ denote the maximal COSET model associated with this inclusion. Then there are unitary, projective representations $\Upsilon^{\mathcal{B}}$ of Diff $\left(\mathrm{S}^{1}\right)$ and $\Upsilon^{\mathcal{A}}, \Upsilon^{\mathcal{C}}$ of Diff, $\left(\mathrm{S}^{1}\right)^{\sim}$ having the following properties:

○ It holds true: $\Upsilon^{\mathcal{B}} \circ \mathrm{p}(\tilde{\varphi})=\Upsilon^{\mathcal{A}}(\tilde{\varphi}) \Upsilon^{\mathcal{C}}(\tilde{\varphi}), \tilde{\varphi} \in \operatorname{Diff}_{+}\left(\mathrm{S}^{1}\right)^{\sim}$.

- For $\varphi \in \operatorname{Diff}_{I}\left(\mathrm{~S}^{1}\right), I \Subset \mathrm{S}^{1}$, we have:

$$
\Upsilon^{\mathcal{B}}(\varphi) \in \mathcal{B}(I), \Upsilon^{\mathcal{A}}\left(\mathrm{p}^{-1}(\varphi)\right) \in \mathcal{A}(I), \Upsilon^{\mathcal{C}}\left(\mathrm{p}^{-1}(\varphi)\right) \in \mathcal{C}_{\max }(I)
$$

- For all elements $\tilde{g}$ of the universal covering group of global conformal transformations, $P S L(2, \mathbb{R})^{\sim}$, holds true:

$$
\Upsilon^{\mathcal{B}}(\mathrm{p}(\tilde{g}))=U(\mathrm{p}(\tilde{g})), \begin{aligned}
& \Upsilon^{\mathcal{A}}(\tilde{g})=U^{\mathcal{A}}(\tilde{g}), \\
& \Upsilon^{\mathcal{C}}(\tilde{g})=U^{\mathcal{A}^{\prime}}(\tilde{g})=U^{\mathcal{C}_{\max }}(\tilde{g}) .
\end{aligned}
$$

Proof: The representations of current algebras induced by embeddings $\iota(\mathfrak{h}) \subset$ $\mathfrak{g}, \mathfrak{h}, \mathfrak{g}$ reductive colour algebras, are known to be completely reducible into 
irreducible highest-weight representations of the current algebra associated with $\iota(\mathfrak{h})$; this yields branchings of the vacuum representation space $\mathscr{H}$ of the current algebra associated with $\mathfrak{g}$ of the form [Kac90, §12.12] [KP84, §4.9] [KW88]:

$$
\mathscr{H}=\bigoplus_{\Lambda} \mathscr{H}_{(\Lambda)} \otimes \mathscr{H}_{\Lambda}
$$

Here $\mathscr{H}_{(\Lambda)}$ is the multiplicity space of the highest-weight representation $\pi_{\Lambda}$ living on $\mathscr{H}_{\Lambda}$; the corresponding representations are of the form $\mathbb{1}_{(\Lambda)} \otimes \pi_{\Lambda}($.$) .$

The HiLBERT space $\mathscr{H}$ is completely reducible into irreducible highest-weight representations with respect to the action of the SUGAWARA stress-energy tensor $\Theta^{\mathcal{B}}$ of $\mathcal{B}$ and the representation spaces $\mathscr{H}_{\Lambda}$ are completely reducible into highest-weight representations with respect to the stress-energy tensor $\Theta^{\mathcal{A}}$ of $\mathcal{A}$ [Kac90, §11.12]. These representations exponentiate to projective representations of Diff $_{+}\left(\mathrm{S}^{1}\right)^{\sim}$ [GW85, TL99]. In the latter reference it is shown that the cocycles of the irreducible, exponentiated highest-weight representations stemming from $\Theta^{\mathcal{B}}$ all coincide. Therefore, we can take their direct sum which defines a projective representation of $\operatorname{Diff}_{+}\left(\mathrm{S}^{1}\right)^{\sim}$; we denote this representation by $\Upsilon^{\mathcal{B}}$. Mutatis mutandis, the same is true for the direct sum of irreducible, projective representations of Diff $\left(\mathrm{S}^{1}\right)^{\sim}$ stemming from $\Theta^{\mathcal{A}}$, which we write as $\Upsilon^{\mathcal{A}}$.

Because the spectrum of the conformal Hamiltonian, the zeroth mode of $\Theta^{\mathcal{B}}$, is integer, it is clear that the kernel of $\mathrm{p}$, the cyclic group generated by $\tilde{R}(2 \pi)$, is represented trivially in $\Upsilon^{\mathcal{B}}$. Thus, $\Upsilon^{\mathcal{B}}$ is a projective representation of $\operatorname{Diff}_{+}\left(\mathrm{S}^{1}\right)$.

The exponentiated self-adjoint integrals of the stress-energy tensor represent the one-parameter groups in $\Upsilon^{\mathcal{B}}$ and $\Upsilon^{\mathcal{A}}$, respectively [Lok94, proposition I.1.13]. HAAG duality of $\mathcal{B}$ and linear energy bounds (theorem [II.1, [BSM90]) imply that localised one-parameter groups of diffeomorphisms are represented in $\Upsilon^{\mathcal{B}}, \Upsilon^{\mathcal{A}}$ by local operators. Moreover, $\Upsilon^{\mathcal{A}}$ commutes with the cyclic projection $e_{\mathcal{A}}$ onto $\overline{\mathcal{A} \Omega}$ because of the SUGAWARA construction; modular covariance of the subnet $\mathcal{A} \subset \mathcal{B}$ implies that localised one-parameter groups of diffeomorphisms are represented in $\Upsilon^{\mathcal{A}}$ by local observables in $\mathcal{A}$. According to [Lok94, proposition V.2.1], products of one-parameter groups in $\operatorname{Diff}_{I}\left(\mathrm{~S}^{1}\right)$ are dense in $\operatorname{Diff}_{I}\left(\mathrm{~S}^{1}\right)$, which proves the statement in (III.39) for $\Upsilon^{\mathcal{B}}$ and $\Upsilon^{\mathcal{A}}$.

The Hermitian linear combinations of the modes $L_{0, \pm 1}^{\Theta^{\mathcal{A}}}$ of $\Theta^{\mathcal{A}}$ satisfy linear energy bounds by arguments as in [BSM90] and integrate to a representation of $P S L(2, \mathbb{R})^{\sim}$ [Frö77], which we know to be globally inner in $\mathcal{A}$ and which has the same infinitesimal action as $U^{\mathcal{A}}$ on the currents in $\mathcal{A}$. Denoting the corresponding linear combinations of generators of $U^{\mathcal{A}}$ by $L_{0, \pm 1}^{\mathcal{A}}$, we recognise that $L_{i}^{\mathcal{A}}-L_{i}^{\Theta^{\mathcal{A}}}$, $i=0, \pm 1$, defines an abelian and hence trivial representation of $\mathfrak{s l}(2)$. This yields the statement on $\Upsilon^{\mathcal{A}}$ in (III.40). By the same line of argument we see that $U$ and $\Upsilon^{\mathcal{B}}$ coincide for global conformal transformations.

The Coset Hamiltonian, $L_{0}-L_{0}^{\mathcal{A}}$, defines an energy grading on the multiplicity spaces $\mathscr{H}_{(\Lambda)}$ with finite-dimensional energy eigenspaces. Hence we know that the $\mathscr{H}_{(\Lambda)}$ are completely reducible into highest-weight representations with 
respect to the action of the CosET stress-energy tensor [Kac90, §11.12]. These representations yield a projective, unitary representation $\Upsilon^{\mathcal{C}}$ of $\operatorname{Diff} f_{+}\left(\mathrm{S}^{1}\right)^{\infty}$ (see arguments above on $\Upsilon^{\mathcal{A}}$ ). In order to show the statement in (III.39), it is sufficient to look at localised one-parameter groups for which it is seen to hold immediately. The statement concerning $\Upsilon^{\mathcal{C}}$ in (III.40) is obvious. The identity $\Upsilon^{\mathcal{B}} \circ \mathrm{p}=\Upsilon^{\mathcal{A}} \Upsilon^{\mathcal{C}}$ follows from the uniqueness of the exponentiation and from $\Theta^{\mathcal{B}}=\Theta^{\mathcal{A}}+\left(\Theta^{\mathcal{B}}-\Theta^{\mathcal{A}}\right)$.

Remarks: Chiral current algebras are known to be strongly additive [TL97, corollary IV.1.3.3], [HL82, BSM90] which means that the local algebras of the maximal Coset model $\mathcal{C}_{\max }(I)$ associated with a current subalgebra coincide with the respective local relative commutants $\mathcal{C}_{I}$. If the CoseT stress-energy tensor has central charge $c \leq 1$, the CoseT model generated by $\Upsilon^{\mathcal{C}}$ is strongly additive [KL02, Xu03]. In this case, the subnet $\mathcal{A}_{\max }$ (see equation II.22) is automatically given by the local relative commutants of $\mathcal{C}_{\max }$ (lemma II.4).

If the CosET central charge is greater than 1 , the subnet generated by $\Upsilon^{\mathcal{C}}$ is not strongly additive [BSM90]. The discussion of the following chapter applies directly to all COsET models of current subalgebras containing $\Upsilon^{\mathcal{C}}$, but the arguments indicate that our analysis probably admits extensions to subnets which do not possess a stress-energy tensor at all (cf discussion in chapter VII).

\section{Conformal covariance subalgebras}

Conformal inclusions of chiral current algebras are of interest for a large variety of reasons. Their classification was undertaken some time ago, because they are particularly relevant to string theory: they make string compactification possible without altering conformal covariance. Using general arguments this task was transferred to checking maximal inclusions of reductive LIE algebras in simple LIE algebras, for which a classification was available already, mainly due to the work of Dynkin [Dyn57a, Dyn57b]. The classification of conformal inclusions was thus achieved, looking at the central charge of the respective stress-energy tensors, by several authors [AGO87, BB87, SW86].

Many of the conformal inclusions were found to correspond to symmetric spaces (cf [GNO85, Dab96] in particular), and isotropy irreducibility of the coset space proved a useful yet neither necessary nor sufficient criterion for an inclusion being conformal. We undertake a complete characterisation of conformal inclusions by means of straightforward arguments familiar in (axiomatic) quantum field theory. On the course we prove a longstanding 6 conjecture of SCHELLEKENS AND WARNER [SW86].

We use properties of any Wightman quantum field theory: positivity of energy, separating property of the vacuum for local quantum fields, and unitarity. Our analysis clarifies the situation in natural group theoretical terms and in direct

\footnotetext{
${ }^{6}$ To the author's surprise, there does not seem to be a proof available yet.
} 
correspondence to quantum field theoretical notions. In addition, there is no need to specialise in maximal subalgebras and our approach is rather direct in that respect.

The methods applied here arise from a more general question: how does the inner-implementing representation $U^{\mathcal{A}}$, uniquely associated with every covariant subtheory $\mathcal{A}$ of a chiral conformal theory $\mathcal{B}$ by means of the BorchersSUGAWARA construction (section II 2), act on the observables of the ambient theory $\mathcal{B}$ ? While detailed knowledge of the action of the SUGAWARA stress-energy tensor of a current subalgebra on the currents of the larger current algebra (equation III.42) does not directly lead to an understanding of the geometric character of this action, it is helpful for characterising the currents on which this action implements conformal covariance. The geometrical impact is resolved in chapter IV in a broader setting.

We proceed as follows: In the following subsection we introduce notations and conventions, prove the conjecture of SCHELLEKENS AND WARNER and provide a direct argument for conformal inclusions being necessarily restricted to level 1. The second subsection is about studying conformal covariance subalgebras associated to LIE algebra inclusions, these being intermediate to the original inclusion, if not trivial. The section will be closed by a simple characterisation of Coset currents, ie current subalgebras commuting with the given current subalgebra. The contents of this section are available as [Kös03b].

\subsection{Characterisation of conformal inclusions}

We study a current algebra with colours in a simple 7 , compact LIE algebra $\mathfrak{g}$ as quantum fields on the chiral light-ray. Basis elements of $\mathfrak{g}$ will be denoted by $T^{a}$; they give the colour of the corresponding current $j^{a}$. The current algebra is given by the following commutation relations:

$$
\left[j^{a}(x), j^{b}(y)\right]=i f^{a b}{ }_{c} j^{c}(x) \delta(x-y)+k g_{\mathfrak{g}}^{a b} \frac{i}{2 \pi} \delta^{\prime}(x-y) .
$$

$g_{\mathfrak{g}}$ denotes the KILLING metric of $\mathfrak{g}, f^{a b}{ }_{c}$ its structure constants and $k$ the current algebra's level; $k$ is a positive integer.

By embedding a reductive LIE subalgebra $\mathfrak{h}$ into $\mathfrak{g}$ via an injective homomorphism $\iota: \mathfrak{h} \hookrightarrow \mathfrak{g}$ we have an associated current subalgebra. $\mathfrak{h}$ consists of several simple ideals, denoted (for the time being) by $\mathfrak{h}_{\alpha}$, and an abelian ideal of dimension $n \geq 0$. The inclusions $\iota\left(\mathfrak{h}_{\alpha}\right) \subset \mathfrak{g}$ are partly characterised by their Dynkin index $I_{\alpha}$, which is defined through the relation $I_{\alpha} g_{\alpha}^{a b}=g_{\mathfrak{g}}^{\iota(a) \iota(b)}$ between the KILLing metric of $\mathfrak{h}_{\alpha}$, denoted $g_{\alpha}$, and the restriction of the KILLING metric of $\mathfrak{g}$ to $\iota\left(\mathfrak{h}_{\alpha}\right)$. The commutation relations (III.5) take the following form for

\footnotetext{
${ }^{7}$ General reasoning leads to an extension of the following discussion to inclusions of reductive subalgebras in reductive LIE algebras, cf eg [AGO87].
} 
currents associated with colours in $\iota\left(\mathfrak{h}_{\alpha}\right)$ :

$$
\left[j^{\iota(a)}(x), j^{\iota(b)}(y)\right]=i f_{\iota(c) \iota(b)}^{\iota{ }^{\iota(c)}}(x) \delta(x-y)+I_{\alpha} k g_{\alpha}^{a b} \frac{i}{2 \pi} \delta^{\prime}(x-y) .
$$

The infinitesimal conformal transformations are implemented by the adjoint action of the SugAWARA stress-energy tensor $\Theta^{\mathfrak{g}}$, see equation (III.11). Restricting to colours in $\iota\left(\mathfrak{h}_{\alpha}\right)$ the SugAWARA stress-energy tensor $\Theta^{\alpha}$ has the same commutation relations with currents associated with colours in $\iota\left(\mathfrak{h}_{\alpha}\right)$ as $\Theta^{\mathfrak{g}}$ :

$$
\Theta^{\alpha}(x)=\frac{\pi}{I_{\alpha} k+\mathrm{g}_{\alpha}^{\vee}} g_{a b}^{\alpha}: j^{\iota(a)} j^{\iota(b)}:(x) .
$$

For the abelian ideal we adopt the following conventions: $I_{\mathbb{R}^{n}}:=1, \mathrm{~g}_{\mathbb{R}^{n}}^{\vee}:=0$, $g_{\mathbb{R}^{n}}^{i j}:=g_{\mathfrak{g}}^{\iota(i) \iota(j)}$. Using these as input all the formulas above apply to currents associated with colours in $\iota\left(\mathbb{R}^{n}\right)$. We shall, therefore, drop the distinction between simple and abelian ideals of $\mathfrak{h}$ and use the symbol $\mathfrak{h}_{\alpha}$ for any simple or the abelian ideal from now on.

With this general notation the action of a stress-energy tensor $\Theta^{\alpha}$ on an arbitrary current $j^{c}$ reads:

$$
\begin{aligned}
{\left[\Theta^{\alpha}(x), j^{c}(y)\right]=} & \frac{\pi}{I_{\alpha} k+\mathrm{g}_{\alpha}^{\vee}} g_{a b}^{\alpha} i f_{d}^{\iota(b) c}: j^{\iota(a)} j^{d}+j^{d} j^{\iota(a)}:(x) \delta(x-y) \\
& +i \frac{k}{I_{\alpha} k+\mathrm{g}_{\alpha}^{\vee}} j^{\iota(a)}(x) g_{a b}^{\alpha} g_{\mathfrak{g}}^{\iota(b) c} \delta^{\prime}(x-y) \\
& +i \frac{1}{2\left(I_{\alpha} k+\mathrm{g}_{\alpha}^{\vee}\right)} j^{d}(x)\left(C_{2}^{\alpha}\right)_{d}^{c} \delta^{\prime}(x-y)
\end{aligned}
$$

This equation is obtained by applying the current algebra and the normal ordering prescription for currents (see equation III.10). The matrix $C_{2}^{\alpha}$ stands for the second CAsimir element of $\mathfrak{h}_{\alpha}$ in the representation $A d_{\mathfrak{g}} \circ \iota \uparrow \mathfrak{h}_{\alpha}$, if $\mathfrak{h}_{\alpha}$ is a simple ideal. In any case we have:

$$
\left(C_{2}^{\alpha}\right)_{d}^{c}=g_{a b}^{\alpha} i f_{d}^{\iota(b) e} i f_{e}^{\iota(a) c}=g_{a b}^{\alpha}\left(A d_{T^{\iota}(b)} A d_{T^{\iota}(a)}\right)_{d}{ }^{c} .
$$

Taking the trace of this matrix one may readily see that it does not vanish for the abelian ideal.

Now we are prepared to state and prove our main result. SCHELLEKEnS AND WARNER conjectured it in their discussion closing [SW86].

Theorem III.4: The following holds true for the weighted Casimir element $\widetilde{C}_{2}^{\iota(\mathfrak{h})}$ of $\iota(\mathfrak{h})\left(P_{\alpha}\right.$ stands for the projection onto $\left.\iota\left(\mathfrak{h}_{\alpha}\right)\right)$ :

$$
\widetilde{C}_{2}^{\iota(\mathfrak{h})}:=\sum_{\alpha} \frac{2 I_{\alpha} k P_{\alpha}+C_{2}^{\alpha}}{2\left(I_{\alpha} k+\mathrm{g}_{\alpha}^{\vee}\right)} \leqslant \mathbb{1} .
$$

This inequality is saturated if and only if $\iota(\mathfrak{h}) \subset \mathfrak{g}$ yields a conformal inclusion, ie $\sum_{\alpha} \Theta^{\alpha}=: \Theta^{\iota(\mathfrak{h})}=\Theta^{\mathfrak{g}}$. 
Proof: By invariance of $g^{\mathfrak{g}}$ the orthocomplementation $\mathfrak{g}=\iota(\mathfrak{h})+\iota(\mathfrak{h})^{\perp}$ provides a reduction of the representation $A d_{\mathfrak{g}} \circ \iota$. We have $C_{2}^{\alpha}\left\lceil\iota(\mathfrak{h})=2 \mathrm{~g}_{\alpha}^{\vee} P_{\alpha}\right.$, ie $\widetilde{C}_{2}^{\iota(\mathfrak{h})} \uparrow$ $\iota(\mathfrak{h})=\mathbb{1}$, and the inequality only remains to be proven for colours orthogonal to $\iota(\mathfrak{h})$, where $P_{\alpha}\left\lceil\iota(\mathfrak{h})^{\perp}=0\right.$. Because all CASIMIR elements commute and all are positive operators, we assume as well that $T^{c}$ is a common eigenvector for all linear mappings $C_{2}^{\alpha}$.

We prove the inequality by looking at specific expectation values of the COSET Hamiltonian $L_{0}^{\mathfrak{g}}-L_{0}^{\mathfrak{h}}$. This is a positive operator, which is given by the Coset stress-energy tensor $\Theta^{\mathfrak{g}}-\Theta^{\iota(\mathfrak{h})}$ smeared with the test function $\xi_{L_{0}}(x)=\frac{1}{2}\left(x^{2}+1\right)$. The infinitesimal action of a conformal Hamilton operator on the test function of a smeared field covariant with respect to it shall be abbreviated by $l_{0}$, ie we have

$$
\left[L_{0}^{\mathfrak{g}}, j^{c}(g)\right]=i \int d x g^{\prime}(x) \xi_{L_{0}}(x) j^{c}(x) \equiv i \int d x\left(l_{0} g\right)(x) j^{c}(x)=i j^{c}\left(l_{0} g\right) .
$$

Using the general commutation relation (III.42), calculating two and three point functions of currents (cf [FST89]), observing that some group-theoretical tensors involved are null for reasons of permutation symmetry/ antisymmetry and carefully taking into account the normal ordering of currents [FST89] one arrives at the following formula:

$$
\begin{aligned}
0 & \leqslant\left\langle\Omega, j^{c}(g)^{\dagger}\left(L_{0}^{\mathfrak{g}}-L_{0}^{\mathfrak{h}}\right) j^{c}(g) \Omega\right\rangle \\
& =i\left(1-\sum_{\alpha} \frac{C_{2}^{\alpha}\left[T^{c}\right]}{2\left(I_{\alpha} k+\mathbf{g}_{\alpha}^{\vee}\right)}\right)\left\langle\Omega, j^{c}(g)^{\dagger} j^{c}\left(l_{0} g\right) \Omega\right\rangle .
\end{aligned}
$$

The desired inequality may be established through division by $i\left\langle\Omega, j^{c}(g)^{\dagger} j^{c}\left(l_{0} g\right) \Omega\right\rangle$, which does not vanish for generic $g$ and is positive as an expectation value of $L_{0} \geq 0$.

If we have $\Theta^{\mathfrak{g}}=\Theta^{\iota(\mathfrak{h})}$, (III.43) is saturated on $\iota(\mathfrak{h})$ trivially $\left(C_{2}^{\alpha} P_{\beta}=2 \mathrm{~g}_{\alpha}^{\vee} \delta^{\alpha}{ }_{\beta}\right)$ and because of (III.44) on $\iota(\mathfrak{h})^{\perp}$ as well, hence on all of $\mathfrak{g}$. The conclusion in the opposite direction is, actually, a consequence of equation (III.45) in proposition III.6: This leads to trivial commutation relations for $\Theta^{\mathfrak{g}}-\Theta^{\iota(\mathfrak{h})}$, especially to $c_{\mathfrak{g}}=c_{\mathfrak{h}}$, which yields, by the REEH-Schlieder theorem, $\Theta^{\mathfrak{g}}-\Theta^{\iota(\mathfrak{h})}=0$.

Corollary III.5: An embedding $\iota(\mathfrak{h}) \subset \mathfrak{g}$ can give rise to a conformal inclusion of the associated current algebras only, if the current algebra associated with $\mathfrak{g}$ has level $k=1$.

Proof: Highest-weight representations of current algebras may be characterised uniquely by a vector of lowest energy which is a highest-weight vector with respect to the horizontal subalgebra. We look at the representation defined by the highest weight $\psi_{\mathfrak{g}}$ of the adjoint representation of $\mathfrak{g}$. Since $\psi_{\mathfrak{g}}$ has, by the usual convention, length 2 , this representation is in accordance with the WEYL-alcove condition 
[FST89, (4.51)] for unitary representations of current algebras for $k \geq 2$. The following argument applies, therefore, to all but level 1.

Actually, we may restrict attention to the action of $L_{0}^{\mathfrak{g}}-L_{0}^{\mathfrak{h}}$ on $\mathfrak{g} \psi_{\mathfrak{g}}$, the highest weight module of $\mathfrak{g}$ generated from the vector with lowest energy and highest weight $\psi_{\mathfrak{g}}$. Here we have (cf equation III.30):

$$
0 \leqslant\left(L_{0}^{\mathfrak{g}}-L_{0}^{\mathfrak{h}}\right)\left\lceil\mathfrak{g} \psi_{\mathfrak{g}}=\frac{\mathrm{g}_{\mathfrak{g}}^{\vee}}{k+\mathrm{g}_{\mathfrak{g}}^{\vee}} \mathbb{1}-\sum_{\alpha} \frac{C_{2}^{\alpha}}{2\left(I_{\alpha} k+\mathrm{g}_{\alpha}^{\vee}\right)} .\right.
$$

This implies a strictly sharper bound than (III.43) and by theorem III.4 this immediately yields the desired result.

\subsection{Covariant and invariant colours}

After we have given a characterisation of conformal inclusions $\iota(\mathfrak{h}) \subset \mathfrak{g}$, we now pursue further the structures in colour space which are associated with the action of $\Theta^{\iota(\mathfrak{h})}$ on currents with colours in $\mathfrak{g}$. We find that covariant and invariant colours form reductive LIE algebras, the first being intermediate to the original embedding $\iota(\mathfrak{h}) \subset \mathfrak{g}$, the second being orthogonal to and commuting with it. All these results are in terms on the weighted CASIMIR element $\widetilde{C}_{2}^{\iota(\mathfrak{h})}$ of the LIE algebra $\iota(\mathfrak{h})$.

The following is the main ingredient of the results in this section:

Proposition III.6: For an arbitrary colour $T^{c} \in \mathfrak{g}$ we have:

$$
\begin{aligned}
& \left\|\left[\left(\Theta^{\mathfrak{g}}-\Theta^{\iota(\mathfrak{h})}\right)(f), j^{c}(g)\right] \Omega\right\|^{2} \\
= & 8 k \pi^{2}\left\langle\left(\mathbb{1}-\widetilde{C}_{2}^{\mathfrak{h}}\right) T^{c}, \widetilde{C}_{2}^{\mathfrak{h}} T^{c}\right\rangle_{\mathfrak{g}} \widetilde{\Delta}^{4}(\overline{f \cdot g}, f \cdot g) \\
& +k\left\langle\left(\mathbb{1}-\widetilde{C}_{2}^{\mathfrak{h}}\right) T^{c},\left(\mathbb{1}-\widetilde{C}_{2}^{\mathfrak{h}}\right) T^{c}\right\rangle_{\mathfrak{g}} \widetilde{\Delta}^{2}\left(\overline{f \cdot g^{\prime}}, f \cdot g^{\prime}\right) .
\end{aligned}
$$

Here $\langle., .\rangle_{\mathfrak{g}}$ stands for the scalar product on $\mathfrak{g}$ induced by the KILLING form. We define:

$$
\Phi^{c}(x):=\sum_{\alpha} \frac{1}{2\left(I_{\alpha} k+\mathrm{g}_{\alpha}^{\vee}\right)} g_{a b}^{\alpha} f_{d}^{\iota(b) c}: j^{\iota(a)} j^{d}+j^{d} j^{\iota(a)}:(x)
$$

The two-point function of $\Phi^{c}$ is given by:

$$
\left\langle\Omega, \Phi^{c}(x) \Phi^{c}(y) \Omega\right\rangle=2 k \widetilde{\Delta}^{4}(x-y)\left\langle\left(\mathbb{1}-\widetilde{C}_{2}^{\mathfrak{h}}\right) T^{c}, \widetilde{C}_{2}^{\mathfrak{h}} T^{c}\right\rangle_{\mathfrak{g}} .
$$

The numerical distributions in these formulae are given by:

$$
\begin{aligned}
\widetilde{\Delta}^{4}(\overline{f \cdot g}, f \cdot g) & =(2 \pi)^{-4} \iint d x d y(i[(x-y)-i \varepsilon])^{-4} \overline{f \cdot g}(x) f \cdot g(y), \\
\widetilde{\Delta}^{2}\left(\overline{f \cdot g^{\prime}}, f \cdot g^{\prime}\right) & =(2 \pi)^{-2} \iint d x d y(i[(x-y)-i \varepsilon])^{-2} \overline{f \cdot g^{\prime}}(x) f \cdot g^{\prime}(y) .
\end{aligned}
$$


Proof: We will not give the derivation of these formulae in detail. We rather indicate their verification. First, one may restrict attention to colours $T^{c} \in$ $\iota(\mathfrak{h})^{\perp}$ since the weighted CASIMIR respects the orthogonal decomposition $\mathfrak{g}=$ $\iota(\mathfrak{h}) \oplus \iota(\mathfrak{h})^{\perp}$ with respect to $A d \circ \iota$ and $\Theta^{\mathfrak{g}}-\Theta^{\iota(\mathfrak{h})}$ commutes with all currents whose colours are in $\iota(\mathfrak{h})$. "All" that one has to do is to apply the general commutation relation (III.42) restricted to colours from $\iota(\mathfrak{h})^{\perp}$, follow carefully the normal ordering of currents, observe symmetries of group theoretical coefficients, keep in mind $T^{c} \in \iota(\mathfrak{h})^{\perp}$, calculate some $n$-point functions of currents following the scheme in [FST89], use JACOBI's identity a few times and recognise the second CASIMIR element in the adjoint representation, which amounts to twice the dual COXETER number. With all that, it is a straightforward algebraic exercise.

Taking $g$ as the test function of constant value 1, equation (III.45) implies $\widetilde{C}_{2}^{\mathfrak{h}}\left(\mathbb{1}-\widetilde{C}_{2}^{\mathfrak{h}}\right) \geq 0$, from which we immediately get inequality (III.43), and the second statement in theorem III.4 follows from (III.45), too.

Definition III.7: $A$ current $j^{c}$ is said to transform covariantly with respect to $\Theta^{\iota(\mathfrak{h})}$, if and only if $\left[\Theta^{\mathfrak{g}}(f), j^{c}(g)\right]=\left[\Theta^{\iota(\mathfrak{h})}(f), j^{c}(g)\right] \forall f, g$.

The proof of the following corollary shows that, in fact, it is sufficient to require the equality to hold for a few, but sufficiently many test functions: two suitable pairs $\left(f_{i}, g_{i}\right), i=1,2$, are enough.

Corollary III.8: A current $j^{c}$ transforms covariantly with respect to $\Theta^{\iota(\mathfrak{h})}$, if and only if its colour fulfills: $\widetilde{C}_{2}^{\iota(\mathfrak{h})} T^{c}=T^{c}$. These covariant colours form a reductive LIE algebra, $\mathfrak{k}$, containing $\iota(\mathfrak{h})$ as a subalgebra. The currents with colours in $\mathfrak{k}$ form the conformal covariance subalgebra. If $\mathfrak{k} \neq \iota(\mathfrak{h})$, then the level of the current algebra associated with $\mathfrak{g}$ has to be $k=1$.

Proof: If we have $\widetilde{C}_{2}^{(\mathfrak{h})} T^{c}=T^{c}$, we know from the variant of the ReEHSchlieder theorem (eg [Jos65, lemma 2, section V.3.B]) and proposition III.6 above, that $j^{c}$ and the Coset stress-energy tensor commute. This is another way of saying: $j^{c}$ transforms covariantly with respect to $\Theta^{\iota(\mathfrak{h})}$.

Conversely: If $j^{c}$ is covariant with respect to $\Theta^{\iota(\mathfrak{h})}$, the group theoretical scalar products in equation (III.45) have to be zero, since the numerical distributions involved are linearly independent. The second one of these is the norm of ( $\mathbb{1}-$ $\left.\widetilde{C}_{2}^{\iota(\mathfrak{h})}\right) T^{c}$, which makes the equation $\widetilde{C}_{2}^{\iota(\mathfrak{h})} T^{c}=T^{c}$ valid.

Now, if $T^{a}$ and $T^{b}$ are covariant colours, then so is $-i\left[T^{a}, T^{b}\right]$. This becomes clear, if one observes $f^{a b}{ }_{c} j^{c}(g)=-i\left[j^{a}([1]), j^{b}(g)\right]$, where [1] is a constant test function: $[1](x)=1$.

The reductivity of $\mathfrak{k}$ is not difficult to prove, either. $\mathfrak{k}$ is a subspace of $\mathfrak{g}$, endowed with an invariant scalar product, which is given by the restriction of the KILLING form on $\mathfrak{g}$. By invariance of this scalar product on $\mathfrak{k}$ with respect to $A d_{\mathfrak{k}}$ (this being a mere restriction of invariance under $A d_{\mathfrak{g}}$ ) any invariant subspace of 
$\mathfrak{k}$ has an invariant orthogonal complement. Now this is complete reducibility of $A d_{\mathfrak{k}}$ and by [Cor89, 25.3.a] $\mathfrak{k}$ is reductive.

Since one can reduce the problem of understanding all conformal inclusions to the studies of reductive inclusions in simple LIE algebras (cf eg [AGO87]) the last part of the claim follows immediately from corollary III.5, as $\iota(\mathfrak{h}) \subset \mathfrak{k}$ is, by construction of $\mathfrak{k}$, a conformal inclusion and the DYNKIN indices of the simple ideals in $\mathfrak{k}$ are greater than or equal to 1 .

Corollary III.9: A current $j^{c}$, whose colour $T^{c}$ lies in $\iota(\mathfrak{h})^{\perp}$ and fulfills $\widetilde{C}_{2}^{\iota(\mathfrak{h})} T^{c}=$ 0 , commutes with the entire current algebra associated with $\iota(\mathfrak{h})$. These colours form a reductive LIE algebra, the algebra of invariant colours; we call their current algebra Coset current algebra.

Proof: We set $V_{0}:=\operatorname{ker}\left(\widetilde{C}_{2}^{\iota(\mathfrak{h})}\right) \cap \iota(\mathfrak{h})^{\perp} . V_{0}$ is an invariant subspace with respect to the action of $\mathfrak{h}$ on $\mathfrak{g}$ via $A d_{\mathfrak{g}} \circ \iota$. In fact, it is the representation space for the trivial subrepresentation on $\iota(\mathfrak{h})^{\perp}$ : For any simple ideal $\mathfrak{h}_{\alpha}$ we have by complete reducibility $C_{2}^{\alpha} \uparrow V_{0}=\sum_{\Lambda}\langle\Lambda+2 \rho, \Lambda\rangle_{\mathfrak{h}_{\alpha}}=0$. Since both the WEYL vector $\rho$ and the contributing highest weight vectors $\Lambda$ are dominant, we have $\Lambda=0$. For the abelian ideal the irreducible subrepresentations on $V_{0}$ are given by common eigenvectors, such that $C_{2}^{\mathbb{R}^{n}} v=g_{\iota(i) \iota(j)}^{\mathfrak{g}} \lambda^{i} \lambda^{j} v=0$. This gives the same result. This means, that all of $V_{0}$ commutes with $\iota(\mathfrak{h})$, ie $V_{0} \subset \iota(\mathfrak{h})^{\prime}$. We gain directly: $V_{0}=\iota(\mathfrak{h})^{\prime} \cap \iota(\mathfrak{h})^{\perp}$.

By JACOBI's identity and invariance of the KILLING metric, $\iota(\mathfrak{h})^{\prime} \cap \iota(\mathfrak{h})^{\perp}$ forms a LIE subalgebra of $\mathfrak{g}$. This is reductive by the same argument as in the proof to corollary III.8.

Generically, the Coset theory is not generated by COSET currents, although there are examples of this structure [KNS88]. Obviously $\iota(\mathfrak{h}) \oplus\left(\iota(\mathfrak{h})^{\prime} \cap \iota(\mathfrak{h})^{\perp}\right) \subset$ $\mathfrak{g}$ has to be a conformal inclusion for that to be the case, since the CoseT stress-energy tensor has to be the SUGAWARA stress-energy tensor of the current algebra associated with $\iota(\mathfrak{h})^{\prime} \cap \iota(\mathfrak{h})^{\perp}$. CAsimir elements of $\iota(\mathfrak{h})^{\prime} \cap \iota(\mathfrak{h})^{\perp}$ give, when transferred to the corresponding horizontal subalgebra, charge operators of the Coset theory. These will, in general, fail to separate the representations of the Coset theory. The same goes for the CARTAN subalgebra of $\iota(\mathfrak{h})^{\prime} \cap \iota(\mathfrak{h})^{\perp}$, whose spectrum defines characters of the representations of the CosET theory. The CoseT current algebra is trivial for all inclusions with minimal CoseT theory: Here the Coset theory is generated by the Coset stress-energy tensor and this theory contains nothing but this field [Car98]. Triviality of CoseT current algebra ought to be regarded as the generic situation.

Currents $j^{c}$ leading to vanishing $\Phi^{c}$ are linear combinations of covariant and Coset currents (proposition III.6). This is obvious, since a decomposition of $T^{c}$ 
into eigenvectors of $\widetilde{C}_{2}^{\mathfrak{h}}$ with distinct eigenvalues $\lambda$ yields:

$$
\left\langle\left(\mathbb{1}-\widetilde{C}_{2}^{\mathfrak{h}}\right) T^{c}, \widetilde{C}_{2}^{\mathfrak{h}} T^{c}\right\rangle_{\mathfrak{g}}=\sum_{\lambda} \lambda(1-\lambda)\left\langle T_{\lambda}^{c}, T_{\lambda}^{c}\right\rangle_{\mathfrak{g}}
$$

As $0 \leq \lambda \leq 1$ this scalar product vanishes, if and only if just 0 and 1 contribute. This means, that there are no currents with a "simple" intermediate transformation behaviour with respect to the action of $\Theta^{\iota(\mathfrak{h})}$. Typically, a current $j^{c}$ has $\Phi^{c} \neq 0$, ie a "complicated" transformation behaviour. By the analysis in chapter IV this behaviour will be seen to be physically satisfactory, still. 


\section{Chapter IV}

\section{Local Nature of COSET Models}

In this chapter we discuss the action of the inner-implementing representation $U^{\mathcal{A}}$ of a chiral subnet $\mathcal{A} \subset \mathcal{B}$ on general local observables in $\mathcal{B}$. The first section studies the "geometric impact" of $U^{\mathcal{A}}$, ie the localisation of $A d_{U^{\mathcal{A}}(\tilde{g})} \mathcal{B}(I)$ depending on $I \Subset \mathrm{S}^{1}$ and $\tilde{g} \in P S L(2, \mathbb{R})^{\sim}$. Intuitively, we do not expect an observable of $\mathcal{B}$ to be more sensitive to the action of $A d_{U \mathcal{A}}$ than to that of $A d_{U}$ : the generator of translations, $P$, is known to decompose into two commuting positive parts, $P=P^{\mathcal{A}}+P^{\mathcal{A}^{\prime}}$, and regarding them as chiral analogues of Hamiltonians leads us to the expectation that $P^{\mathcal{A}}$ should not transport observables of $\mathcal{B}$ "faster" than $P$ itself. A typical observable $B$ in $\mathcal{B}$ should exhibit a behaviour interpolating between invariance $\left(B\right.$ in $\mathcal{C}_{\max }$ ) and covariance $\left(B\right.$ in $\left.\mathcal{A}_{\max }\right)$.

For this behaviour to be ensured we have, as it turns out, only to show that scale transformations represented through $U^{\mathcal{A}}$ respect the two fixed points of scale transformations, namely 0 and $\infty$, when acting on $\mathcal{B}$. We can prove this to be the case in presence of a stress-energy tensor and it seems natural in any case. The sub-geometrical transformation behaviour for translations, which we expect, then follows by results of Borchers [Bor97a, Bor97b] using the spectrum condition and modular theory. We collected, rearranged and reformulated results of BORCHERS and WIESBROCK in order to provide a natural converse of Borchers' theorem on half-sided translations, which was not yet available in the literature. By extending the analysis to general conformal transformations we arrive at the notion of net-endomorphism property for the action of $U^{\mathcal{A}}$ on $\mathcal{B}$.

In the second section we use the net-endomorphic action of $U^{\mathcal{A}}$ to construct from the chiral conformal theory $\mathcal{B}$ a conformal net in $1+1$ dimensions which contains the chiral algebras as time-zero algebras. The result satisfies all axioms of a 1+1-dimensional conformal quantum theory, except that not translations in futurelike directions have positive spectrum but rather translations in right spacelike directions. While this prohibits interpreting the picture of chiral holography as genuinely physical, it provides a helpful geometrical framework of a quasi-theory in $1+1$ dimensions. The maximal CoseT model appears as a subtheory of chiral observables and hence we make contact with results of REHREN 
[Reh00], which have interesting consequences for known examples.

In the last section we provide our solution to the isotony problem (main theorem IV.13), ie we establish the local nature of the maximal CoseT model. We start by giving a new characterisation of $\mathcal{C}_{\max }$ making use of the particular structure of the group of chiral conformal transformations. And then, again, the presence of a stress-energy tensor for $\mathcal{A}$ is only needed in order to establish a rather natural, but crucial lemma on the representation of scale transformations through $U^{\mathcal{A}}$. Most of this chapter is available as [Kös03c].

\section{Net-endomorphism Property}

In the following we deduce, step by step, the sub-geometric character of the adjoint action of $U^{\mathcal{A}}$ (and of $U^{\mathcal{A}^{\prime}}$ ) on $\mathcal{B}$. The analysis relies on a single property of the dilatations in $U^{\mathcal{A}}$. The notion of net-endomorphisms arises naturally in the course of the argument and will be discussed at the end of this section. We, therefore, define:

Definition IV.1: $U^{\mathcal{A}}$ is said to have the net-endomorphism property, if the adjoint action of $U^{\mathcal{A}}(\tilde{D}(t)), t \in \mathbb{R}$, defines a group of automorphisms of $\mathcal{B}\left(\mathrm{S}_{+}^{1}\right)$.

The net-endomorphism property holds making the following

Additional Assumption: There is a unitary, strongly continuous, projective representation $\Upsilon^{\mathcal{A}}$ of the universal covering group of orientation preserving diffeomorphisms of the circle, Diff $\left(\mathrm{S}^{1}\right)^{\sim}$, on $\mathscr{H}$ such that:

- If a diffeomorphism $\varphi \in$ Diff $_{+}\left(\mathrm{S}^{1}\right)$ is localised in $I \Subset \mathrm{S}^{1}$, ie $\varphi \uparrow I^{\prime}=i d \uparrow I^{\prime}$, it is represented by a local observable of $\mathcal{A}$, namely: $\Upsilon^{\mathcal{A}}\left(\mathrm{p}^{-1}(\varphi)\right) \in \mathcal{A}(I)$.

○ $\Upsilon^{\mathcal{A}}(\tilde{D}(t)) U^{\mathcal{A}}(\tilde{D}(t))^{*} \in \mathbb{C}_{1} \mathbb{1}_{\mathscr{H}}$ for all $t \in \mathbb{R}$.

Here, the covering projection from Diff, $\left(\mathrm{S}^{1}\right)^{\sim}$ onto Diff $\left(\mathrm{S}^{1}\right)$ is denoted by $\mathrm{p}$. Localised diffeomorphisms $\varphi$ are identified with their preimage $\mathrm{p}^{-1}(\varphi)$ in the first sheet of the covering.

The Additional Assumption only enters through the lemmas IV.2 and IV.12, which we believe to hold true in a lot more general circumstances. It was verified in presence of an integrable stress-energy tensor in $\mathcal{A}$ for current subalgebras in theorem III.3. In this case the representations $\Upsilon^{\mathcal{A}} \uparrow P S L(2, \mathbb{R})^{\sim}$ and $U^{\mathcal{A}}$ coincide, whereas we have only assumed that the respective generators agree up to a multiple of $\mathbb{1}$. At this point we want to stress: We do not assume $\mathcal{A}$ to be diffeomorphism covariant, ie the adjoint action of $\Upsilon^{\mathcal{A}}$ on $\mathcal{A}$ to implement a geometric, automorphic action of $\operatorname{Diff}_{+}\left(\mathrm{S}^{1}\right)$ on $\mathcal{A}$.

Lemma IV.2: $U^{\mathcal{A}}$ has the net-endomorphism property, if the ADDITIONAL AsSUMPTION holds. 
Proof: By lemma A.4 there exist, for small $t \in \mathbb{R}$, diffeomorphisms $g_{\varepsilon}, g_{\delta}$ localised in arbitrarily small neighbourhoods of -1 and 1 , respectively, and diffeomorphisms $g_{+}, g_{-}$localised in $\mathrm{S}_{+}^{1}$ and $\mathrm{S}_{-}^{1}$, respectively, such that we have: $D(t)=g_{+} g_{-} g_{\delta} g_{\varepsilon}$. If the closure of a proper interval $I$ is contained in $\mathrm{S}_{+}^{1}$, we have with an appropriate choice of $g_{\delta}, g_{\varepsilon}$ by the Additional Assumption :

$$
U^{\mathcal{A}}(\tilde{D}(t)) \mathcal{B}(I) U^{\mathcal{A}}(\tilde{D}(t))^{*}=\Upsilon^{\mathcal{A}}\left(\mathrm{p}^{-1}\left(g_{+}\right)\right) \mathcal{B}(I) \Upsilon^{\mathcal{A}}\left(\mathrm{p}^{-1}\left(g_{+}\right)\right)^{*} \subset \mathcal{B}\left(\mathrm{S}_{+}^{1}\right) .
$$

Because $\mathcal{B}\left(\mathrm{S}_{+}^{1}\right)$ is continuous from the inside, we see that $A d_{U^{\mathcal{A}}(\tilde{D}(t))}$ induces an endomorphism of $\mathcal{B}\left(\mathrm{S}_{+}^{1}\right)$. The same holds true for $U^{\mathcal{A}}(\tilde{D}(-t))$ and, therefore, these endomorphisms are automorphisms.

Remark: From formula (A.3) we readily see that a stress-energy tensor yields lemma IV.2 without any direct reference to the structure of $\operatorname{Diff}_{+}\left(\mathrm{S}^{1}\right)$ or, indeed, the LÜsCHER-MACK algebra (III.13).

The next step is to give a characterisation of one-parameter groups of unitary operators which define, by their adjoint action, endomorphism semigroups of a standard V.NEUMANN algebra. The following theorem is mainly a new formulation of results by BorCHERs and WiEsBROCK. It appears to be a natural converse of Borchers' theorem on half-sided translations. The methods involved in the proof are completely standard, but the result ought to be made available 1 .

Theorem IV.3: Assume $\mathcal{M} \subset \mathcal{B}(\mathscr{H})$ to be a V.NEUMANn algebra having a cyclic and separating vector $\Omega$ in the separable HILBERT space $\mathscr{H}$. J, $\Delta$ shall stand for the modular data of this pair. Let $V(t), t \in \mathbb{R}$, be a strongly continuous one-parameter group. Then any two from $\{i),(i i),(i i i)\}$ imply the remaining two in the list below; iv) yields i), ii), iii).

i) a. $V(s)=e^{i H s}, H \geqslant 0$, b. $V(s) \mathcal{M} V(s)^{*} \subset \mathcal{M}, s \geqslant 0$.

ii) $\quad$ a. $V(s) \Omega=\Omega, s \in \mathbb{R}$, b. $V(s) \mathcal{M} V(s)^{*} \subset \mathcal{M}, s \geqslant 0$.

iii) $\quad$ a. $\Delta^{i t} V(s) \Delta^{-i t}=V\left(e^{-2 \pi t} s\right), J V(s) J=V(-s), t, s \in \mathbb{R}$, b. $V(s) \mathcal{M} V(s)^{*} \subset \mathcal{M}, s \geqslant 0$.

iv) a. $V(s)=e^{i H s}, H \geqslant 0$,

b. $\Delta^{i t} V(s) \Delta^{-i t}=V\left(e^{-2 \pi t} s\right), t, s \in \mathbb{R}$, c. $\left\langle m_{+}^{\prime} \Omega, V(s) m_{+} \Omega\right\rangle \geqslant 0, s \geqslant 0, m_{+} \in \mathcal{M}_{+}, m_{+}^{\prime} \in \mathcal{M}_{+}^{\prime}$.

\footnotetext{
${ }^{1}$ Compare [Dav96] for another characterisation of endomorphism semigroups related to BoRCHERS' theorem.
} 
$\mathcal{M}_{+}$denotes the cone of positive elements in $\mathcal{M}, \mathcal{M}_{+}^{\prime}$ the cone of positive elements in its commutant $\mathcal{M}^{\prime}$.

Proof: Most of the implications were proved by Borchers and WiESBROCK, respectively: i) $\wedge$ ii) $\Rightarrow$ iii): [Bor92] (cf [Flo98]); ii) $\wedge$ iii) $\Rightarrow$ i): [Wie92]; i) $\wedge$ iii) $\Rightarrow$ ii): [Bor98]; i) $\wedge$ ii) $\wedge$ iii) $\Rightarrow$ iv): [BR87, proposition 2.5.27].

We prove the remaining statement, namely iv) $\Rightarrow$ i) $\wedge($ ii) $\wedge$ iii), by reduction to [Bor97a, theorem 1.1] $]_{2}^{2}$. As a first step we look at the domain of entire analytic vectors with respect to $\Delta^{i z}$, which we denote by $D_{\Delta}$, and derive an analytic continuation of relation iv)b. as a quadratic form on $D_{\Delta}$. We define:

$$
F(z, w):=\left\langle\Delta^{i \bar{z}} \psi, e^{i e^{2 \pi w} H} \Delta^{i z} \phi\right\rangle .
$$

According to the spectrum condition on $H, F$ is analytic in $w$ for $0<\operatorname{Im}(w)<\frac{1}{2}$, and this function is bounded and continuous for the closure of this region; the region itself shall be denoted by $\mathbb{S}$. In fact, by HARTOG's theorem, $F$ is analytic on $\mathbb{C} \times \mathbb{S}$ as a function in two complex variables. We make full use of relation iv)b. by looking at another function $G$, which agrees with $F$ for $0<\operatorname{Im}(w)+\operatorname{Im}(z)<\frac{1}{2}$ :

$$
G(z, w):=\left\langle\psi, e^{i e^{2 \pi(w+z)} H} \phi\right\rangle .
$$

Evaluating at $w \in \mathbb{R}$ and $z=\frac{i}{4}$ we get:

$$
\left\langle\Delta^{\frac{1}{4}} \psi, e^{i e^{2 \pi w} H} \Delta^{-\frac{1}{4}} \phi\right\rangle=\left\langle\psi, e^{-e^{2 \pi w} H} \phi\right\rangle .
$$

Both $\psi, \phi$ are of the form $\psi=\Delta^{-\frac{1}{4}} \psi^{\prime}, \phi=\Delta^{\frac{1}{4}} \phi^{\prime}, \psi^{\prime}, \phi^{\prime} \in D_{\Delta}$. Since the set of such $\psi^{\prime}, \phi^{\prime}$ is dense in $\mathscr{H}$, the equation above becomes an equation for bounded operators, which yields:

$$
e^{i s H}=\Delta^{-\frac{1}{4}} e^{-s H} \Delta^{\frac{1}{4}}, \quad s \geqslant 0 .
$$

Next, we show invariance of $\Omega$ following arguments from the proof of [Bor98, lemma 2.3.c]: let $E$ be the projection onto the eigenvectors of $\Delta$ having eigenvalue 1. Multiplying the identity (IV.3) from both sides by $E$ leads to:

$$
E e^{i s H} E=E e^{-s H} E, \quad s \geqslant 0 .
$$

Here, the right hand side is a positive operator and thus we have as well:

$$
\left(E e^{i s H} E\right)^{*}=E e^{-i s H} E=E e^{-s H} E=E e^{i s H} E, \quad s \geqslant 0 .
$$

According to a standard argument 3 , this invariance with respect to conjugation yields: $E e^{i s H} E=E e^{i 0 H} E=E$. Therefore, all vectors $\xi$ satisfying $\xi=E \xi$ are invariant under the action of $V$ and this means in particular: $V(s) \Omega=\Omega, \forall s \in \mathbb{R}$.

\footnotetext{
${ }^{2}$ Alternatively, one may use the same statement in [Bor97b, theorem 2.5].

${ }^{3}$ Such an argument is given, for example, in the proof of corollary II.16 and uses the spectrum condition, the Phragmen-Lindelöf theorem, Schwarz' reflection principle and Liouville's theorem.
} 
It now follows from iv)c. and [BR87, proposition 2.5.28] that $e^{-s H}, s \geq 0$, leaves the natural cone of $(\mathcal{M}, \Omega)$ globally fixed. The other assumptions of [Bor97a, theorem 1.1] are the identities:

$$
\begin{aligned}
\Delta^{i t} e^{-H s} \Delta^{-i t} & =e^{-s e^{-2 \pi t} H}, \quad s \geqslant 0, \\
e^{-H s} \Omega & =\Omega, \quad s \geqslant 0 .
\end{aligned}
$$

These relations are obvious by analytic continuation of results derived above. By [Bor97a, theorem 1.1] the adjoint action of $V(s), s \geq 0$, does indeed induce endomorphisms of $\mathcal{M}$ and we have completed the proof.

The analytic continuation of the dilatation-translation relation iv)b. to imaginary arguments as in (IV.2) is a consequence of the spectrum condition and does not follow from general group theoretical bounds (see eg [BR77, chapter 11, §3, theorem 4]). It does not appear to be possible to drop the assumption iv)a. on the spectrum condition and to deduce it in the course of the argument, since one inevitably runs into domain problems (cf [Dav96, theorem 1], [Lon97, corollary 2.8], [BCL98, proposition 2.4]).

The arguments in the proof of theorem IV.3 apply, with minor alterations, to translation groups with negative generator, as eg the special conformal transformations $U(S()$.$) . While J$ has the same action, $J U(S(n)) J=U(S(-n))$, the scaling behaviour is opposite:

$$
\Delta^{i t} U(S(n)) \Delta^{-i t}=U\left(S\left(e^{2 \pi t} n\right)\right) .
$$

The negative spectrum together with the opposite scaling law (IV.4) shows that the condition characterising endomorphism semi-groups is again given by the one in iv)c. Since the arguments are the same as for the case of positive spectrum and scaling law iii)a., iv)b. we state the following corollary without proof:

Corollary IV.4: The statements in theorem IV.3 still hold, if one uses $V(s)=$ $e^{i K s}, K \leq 0$, instead of i)a., iv)a. and replaces the scaling law in iii)a., iv)b. by $\Delta^{i t} V(s) \Delta^{-i t}=V\left(e^{2 \pi t} s\right), s, t \in \mathbb{R}$.

At this stage our intuition about the geometric impact of $U^{\mathcal{A}}$ on $\mathcal{B}$ can be verified. We will discuss the general situation after the following corollary:

Corollary IV.5: Assume $U^{\mathcal{A}}$ to have the net-endomorphism property. Then the adjoint action of $U^{\mathcal{A}^{\prime}}(\tilde{D}()$.$) on \mathcal{B}\left(\mathrm{S}_{+}^{1}\right)$ defines a group of automorphisms.

For $s \geq 0$ the adjoint action of $U^{\mathcal{A}}(\tilde{T}(s))$ induces endomorphisms of $\mathcal{B}\left(\mathrm{S}_{+}^{1}\right)$ and the adjoint action of $U^{\mathcal{A}}(\tilde{T}(-s))$ maps $\mathcal{B}\left(\mathrm{S}_{+}^{1}\right)$ into $\mathcal{B}\left(T(-s) \mathrm{S}_{+}^{1}\right)$. The corresponding statements hold true, if one replaces $\mathcal{A}$ by $\mathcal{A}^{\prime}$ or $\tilde{T}($.$) by \tilde{S}($.$) .$

Proof: The statement on $A d_{U^{\mathcal{A}^{\prime}}(\tilde{D}(.))}$ follows from $U^{\mathcal{A}^{\prime}}=U$ op $U^{\mathcal{A} *}$ and covariance of $\mathcal{B}$. Using the factorisation $U(T(s))=U^{\mathcal{A}}(\tilde{T}(s)) U^{\mathcal{A}^{\prime}}(\tilde{T}(s))$, covariance and 
isotony of $\mathcal{B}$, the statement on $A d_{U^{\mathcal{A}^{\prime}}(\tilde{D}(.))}$ and invariance of $\Omega$ with respect to $U^{\mathcal{A}^{\prime}}$, we have the following inequality for all $t \in \mathbb{R}, s \geq 0, B_{+} \in \mathcal{B}\left(\mathrm{S}_{+}^{1}\right)_{+}, B_{+}^{\prime} \in \mathcal{B}\left(\mathrm{S}_{-}^{1}\right)_{+}$:

$$
\begin{aligned}
0 & \leqslant\left\langle U^{\mathcal{A}^{\prime}}(\tilde{D}(t))^{*} B_{+}^{\prime} U^{\mathcal{A}^{\prime}}(\tilde{D}(t)) \Omega, U(T(s)) U^{\mathcal{A}^{\prime}}(\tilde{D}(t))^{*} B_{+} U^{\mathcal{A}^{\prime}}(\tilde{D}(t)) \Omega\right\rangle \\
& =\left\langle B_{+}^{\prime} \Omega, U^{\mathcal{A}}(\tilde{T}(s)) U^{\mathcal{A}^{\prime}}\left(\tilde{T}\left(e^{t} s\right)\right) B_{+} \Omega\right\rangle .
\end{aligned}
$$

In the limit $t \rightarrow-\infty$ strong continuity of $U^{\mathcal{A}^{\prime}}$ implies $\left\langle B_{+}^{\prime} \Omega, U^{\mathcal{A}}(\tilde{T}(s)) B_{+} \Omega\right\rangle \geq 0$, which in turn yields the statement on $U^{\mathcal{A}}(\tilde{T}(s)), s \geq 0$, by theorem IV.3, because of the BISOGNANO-Wichmann property of $\mathcal{B}$ and general results on $U^{\mathcal{A}}$ (section II/2). Following the same argument with $\mathcal{A}$ instead of $\mathcal{A}^{\prime}$ and vice versa leads to the corresponding statement on $U^{\mathcal{A}^{\prime}}(\tilde{T}(s)), s \geq 0$. If one replaces in both statements $\tilde{T}(s)$ by $\tilde{S}(s)$, one may apply the argument as well, but using the limit $t \rightarrow \infty$ and corollary IV.4.

The remainder follows immediately from the following argument, which we indicate for the translations represented through $U^{\mathcal{A}}$ :

$$
\begin{aligned}
A d_{U^{\mathcal{A}}(\tilde{T}(-s))} \mathcal{B}\left(\mathrm{S}_{+}^{1}\right) & =A d_{U(T(-s))} A d_{U \mathcal{A}^{\prime}(\tilde{T}(s))} \mathcal{B}\left(\mathrm{S}_{+}^{1}\right) \\
& \subset A d_{U(T(-s))} \mathcal{B}\left(\mathrm{S}_{+}^{1}\right)=\mathcal{B}\left(T(-s) \mathrm{S}_{+}^{1}\right) .
\end{aligned}
$$

The geometric impact of a general $U^{\mathcal{A}}(\tilde{g}), \tilde{g} \in P S L(2, \mathbb{R})^{\sim}$, on an arbitrary local algebra $\mathcal{B}(I)$ is discussed easily. We may restrict our attention to group elements $\tilde{g}$ for which there is a single sheet of the covering projection p containing both $\tilde{g}$ and the identity, as the following discussion indicates.

Every element $g$ in $\operatorname{PSL}(2, \mathbb{R})$ is contained in (at least) one one-parameter group ${ }^{4}$ [Mos94, Mos97]. We use the local identification of one-parameter subgroups in $\operatorname{PSL}(2, \mathbb{R})$ and in $\operatorname{PSL}(2, \mathbb{R})^{\sim}$, choose a parametrisation such that $\tilde{g}=\tilde{g}(1), i d=\tilde{g}(0)$, and we set $\gamma_{\tilde{g}}(I):=\bigcup_{\tau=0}^{1} \mathrm{p}(\tilde{g}(\tau)) I$. For $\tilde{g}$ further away from the identity we set $\gamma_{\tilde{g}}(I):=\mathrm{S}^{1}$ and take $\mathcal{B}\left(\mathrm{S}^{1}\right)$ to be the algebra of all bounded operators on $\mathscr{H}$. Then we have:

Proposition IV.6: Assume $U^{\mathcal{A}}$ to have the net-endomorphism property. Then for any $\tilde{g} \in P S L(2, \mathbb{R})^{\sim}$ and any $I \Subset \mathrm{S}^{1}$ :

$$
A d_{U^{\mathcal{A}}(\tilde{g})} \mathcal{B}(I) \subset \mathcal{B}\left(\gamma_{\tilde{g}}(I)\right), \quad A d_{U^{\mathcal{A}^{\prime}(\tilde{g})}} \mathcal{B}(I) \subset \mathcal{B}\left(\gamma_{\tilde{g}}(I)\right) .
$$

Proof: Each proper interval $I$ in $\mathrm{S}^{1}$ may be identified by the ordered pair consisting of its boundary points, $z_{+}$and $z_{-}$. We define three one-parameter subgroups in $P S L(2, \mathbb{R})$ referring to each $I \Subset \mathrm{S}^{1}$ with respect to a particular choice $h \in P S L(2, \mathbb{R})$ satisfying $h S_{+}^{1}=I: D_{I}()=.h D(.) h^{-1}, T_{I}()=.h T(.) h^{-1}$, $S_{I}()=.h S(.) h^{-1}$.

\footnotetext{
${ }^{4}$ I am indebted to D. Guido for providing the reference. In the particular case of $\operatorname{PSL}(2, \mathbb{R})$ this fact may be checked directly (proposition A.3).
} 
Each element $g$ in $P S L(2, \mathbb{R})$ is fixed, up to a dilatation $D_{I}(t)$, by its action on $\left\{z_{+}, z_{-}\right\}$. Under the action of elements $g(\tau), \tau=0, \ldots, 1$, interpolating in the one-parameter group associated with $g$ between the identity and $g=g(1)$, the orbits of $z_{ \pm}$are given by monotonous functions $z_{+}(\tau), z_{-}(\tau)$. Demanding $s, n, t$ to depend continuously on $\tau$ and to take value 0 at $\tau=0$, every $g(\tau)$ may be represented as $g(\tau)=S_{T_{I}(s(\tau)) I}(n(\tau)) T_{I}(s(\tau)) D_{I}(t(\tau))$ or as $g(\tau)=T_{S_{I}(n(\tau)) I}(s(\tau)) S_{I}(n(\tau)) D_{I}(t(\tau))$. We choose one form which works for all interpolating elements. By the requirements we have made it is ensured that the representation works (after obvious identifications) in $P S L(2, \mathbb{R})^{\sim}$ as well. Corollary IV.5 implies the claim of the proposition now.

This proves in particular: For every $I \Subset \mathrm{S}^{1}$ there is a neighbourhood of the identity in $\operatorname{PSL}(2, \mathbb{R})^{\sim}$ for which the action of $A d_{U^{\mathcal{A}}(.)}$ on $\mathcal{B}(I)$ delivers local observables. The result of this proposition can be improved on grounds of some further assumptions; see proposition V.11.

We have found $A d_{U^{\mathcal{A}}}$ to induce homomorphisms from local algebras of $\mathcal{B}$ into algebras associated with an enlarged localisation region. This sub-geometrical action respects isotony, ie the net-structure. The adjoint action of $U$ induces the covariance isomorphisms of local algebras and one usually regards these as automorphisms of the net $\mathcal{B}$. We consider, therefore, the term net-endomorphisms appropriate. The automorphic action of $A d_{U \mathcal{A}(\tilde{D}(.))}$ on $\mathcal{B}\left(\mathrm{S}_{+}^{1}\right)$ which we proved in lemma IV.2 does not, apparently, follow from the endomorphism property for translations in corollary IV.5. This motivated definition IV.1 above.

\section{Chiral holography}

We give a holographic interpretation of the net-endomorphism property. This shows that the results achieved so far are satisfactory, and it yields new insights into structures associated with chiral conformal subnets and their COSET models.

If we define $U^{\mathcal{A}} \times U^{\mathcal{A}^{\prime}}$ as representation of $\operatorname{PSL}(2, \mathbb{R})^{\sim} \times \operatorname{PSL}(2, \mathbb{R})^{\sim}$ through $(\tilde{g}, \tilde{h}) \mapsto U^{\mathcal{A}}(\tilde{g}) U^{\mathcal{A}^{\prime}}(\tilde{h})$, this is, in fact, a representation of the conformal symmetry group of a local conformal quantum theory in $1+1$ dimensions, which is isomorphic to $\left(P S L(2, \mathbb{R})^{\sim} \times P S L(2, \mathbb{R})^{\sim}\right) / \mathbb{Z}$. This factor group arises, if one identifies the simultaneous rigid conformal rotation by $2 \pi$, namely $(\tilde{R}(2 \pi), \tilde{R}(2 \pi))$, with the trivial transformation. The last section taught us a lot about the subgeometrical action of $U^{\mathcal{A}}, U^{\mathcal{A}^{\prime}}$ on the local observables in $\mathcal{B}$. So, it is natural to look for a relation between the geometrical character of this action and structures in $1+1$ dimensions.

This relation turns out to be a complete correspondence: We construct a $1+1$-dimensional, local, conformal theory from the original chiral theory $\mathcal{B}$ applying the net-endomorphism property of $U^{\mathcal{A}}$. In order to prove locality in $1+1$ dimensions we are led to a particular choice of light-cone coordinates, by which 
the original local algebras $\mathcal{B}(I), I \Subset \mathrm{S}^{1}$, are included in the 1+1-dimensional picture as time zero algebras. This choice of coordinates yields an unphysical spectrum condition: translations in the right spacelike wedge have positive spectrum. Whereas this prohibits an interpretation of the new theory as a genuinely physical one, where we would have positivity of the spectrum in future-like directions, the construction does provide a useful geometrical picture for questions concerned with chiral subnets and their COSET models. For this reason we have to regard the result of our construction as a local, conformal quasi-theory in $1+1$ dimensions.

If, on the opposite, one takes a conformal local quantum theory in $1+1$ dimensions and defines a chiral conformal net by restriction to time zero algebras, a similar phenomenon arises (cf [KLM01, Lon01]): the spectrum condition disappears altogether, but powerful tools of local quantum theory are available still, because the REEH-SCHLIEDER property survives. In our case there remains a spectrum condition from which one can still derive the REEH-SchliEDER property. In this sense we find a natural "converse" of the restriction process which justifies the term chiral holography for our construction.

The main result of this section will be proved by making contact with the analysis of BRUNETti, GuIDo AND LONGO [BGL93] who discussed conformal quantum field theories in general spacetime dimensions as local quantum theories on the conformal covering of the respective MINKOWSKI space given by extensions of local nets living on MinKOWSKI space itself.

\subsection{The holographic quasi-theory in $1+1$ dimensions}

In $1+1$ dimensions, Minkowski space $\mathbb{M}$ is the Cartesian product of two chiral light-rays, which we take as light-cone coordinates of $\mathbb{M}=\mathbb{R} \times \mathbb{R}$. One arrives at the (physical) conformal covering $\widetilde{\mathbb{M}}$ of $\mathbb{M}$, if one compactifies both light-rays adding the points at infinity, takes the infinite, simply connected covering of the compactification $S^{1} \times S^{1}$, which yields $\mathbb{R} \times \mathbb{R}$, and, finally, one identifies all points which are connected by the action of simultaneous rigid conformal rotations by $2 \pi$. The result has the shape of a cylinder having infinite timelike extension: $\widetilde{\mathbb{M}}=$ $\mathrm{S}^{1} \times \mathbb{R}$. Without the final identification we would have spacelike separated copies of $\mathbb{M}$ in covering space, which we consider unphysical; conformally covariant quantum fields can be proven to live on this (physical) conformal covering of MinKOWSKI space, see [LM75].

Light-rays in $\widetilde{\mathbb{M}}$ are infinitely extended, universal coverings of the compactified light-rays and serve well as light-cone coordinates of $\widetilde{\mathbb{M}}$. The localisation regions, which we will consider, are 1+1-dimensional double cones given as Cartesian products of two intervals, $I \times J$, where $I, J$ are properly contained in a single copy of $S^{1}$ on the left and right light-rays in $\widetilde{\mathbb{M}}$, respectively.

$P S L(2, \mathbb{R})^{\sim}$, the universal covering group of the chiral conformal transformations, has an action on the infinite covering $\mathbb{R}$ of $S^{1}$ which is transitive for the 
intervals which are properly contained in a single copy of $\mathrm{S}^{1}$. We exclude the point of infinity from $S^{1}$ and choose a fixed interval $I$ which is properly contained in the remainder. This interval is identified with its first pre-image in covering space. For intervals $J_{L}, J_{R}$ which are properly included in a single copy of $\mathrm{S}^{1}$ we choose group elements $\tilde{g}_{L, R} \in P S L(2, \mathbb{R})^{\sim}$ satisfying $J_{L}=\tilde{g}_{L} I, J_{R}=\tilde{g}_{R} I$. Making use of this choice we define a set of (local) algebras indexed by $1+1$-dimensional double cones:

$$
\mathcal{B}^{1+1}\left(J_{L} \times J_{R}\right):=U^{\mathcal{A}}\left(\tilde{g}_{L}\right) U^{\mathcal{A}^{\prime}}\left(\tilde{g}_{R}\right) \mathcal{B}(I) U^{\mathcal{A}}\left(\tilde{g}_{L}\right)^{*} U^{\mathcal{A}^{\prime}}\left(\tilde{g}_{R}\right)^{*}
$$

By covariance of $\mathcal{B}$, the resulting algebra $\mathcal{B}^{1+1}\left(J_{L} \times J_{R}\right)$ is uniquely determined by $J_{L} \times J_{R}$.

Furthermore, we define a covering projection $\mathrm{p}$ from $\mathbb{R}$ onto $\mathrm{S}^{1}$ referring to the covering projection $\mathrm{p}: P S L(2, \mathbb{R})^{\sim} \rightarrow P S L(2, \mathbb{R})$ such that we have: $\mathrm{p} J_{L, R}:=$ $\mathrm{p}\left(\tilde{g}_{L, R}\right) I$. Because of the close relation between both covering projections and the fact that the respective argument distinguishes between them, we did not introduce a new symbol. This definition enables us to state two identities for the algebras defined in equation (IV.5):

$$
\begin{aligned}
\mathcal{B}^{1+1}\left(J_{L} \times J_{R}\right) & =U^{\mathcal{A}}\left(\tilde{g}_{L} \tilde{g}_{R}{ }^{-1}\right) \mathcal{B}\left(\mathrm{p} J_{R}\right) U^{\mathcal{A}}\left(\tilde{g}_{L} \tilde{g}_{R}{ }^{-1}\right)^{*} \\
& =U^{\mathcal{A}^{\prime}}\left(\tilde{g}_{R} \tilde{g}_{L}{ }^{-1}\right) \mathcal{B}\left(\mathrm{p} J_{L}\right) U^{\mathcal{A}^{\prime}}\left(\tilde{g}_{R} \tilde{g}_{L}{ }^{-1}\right)^{*}
\end{aligned}
$$

Double cones $J \times J$, which are centered at the time zero axis, are called time zero double cones and we get for the corresponding time zero algebras:

$$
\mathcal{B}^{1+1}(J \times J)=\mathcal{B}(\mathrm{p} J)
$$

Thus, the local algebras of the original chiral conformal theory $\mathcal{B}$ are included into the new quasi-theory $\mathcal{B}^{1+1}$ as time zero algebras:

Theorem IV.7: If $\mathcal{A} \subset \mathcal{B}$ is an inclusion of chiral conformal theories and if the unique inner-implementing representation $U^{\mathcal{A}}$ associated with this inclusion has the net-endomorphism property, then equation (IV.5) defines a set $\mathcal{B}^{1+1}$ of local algebras assigned to double cones in $1+1$-dimensional conformal space time, $\widetilde{\mathbb{M}}$, having all but one of the usual properties of a local, conformal, weakly additive quantum theory in $1+1$ dimensions (see [BGL93]): the spectrum condition holds for translations in the right spacelike wedge.

Proof: Obviously, the set $\mathcal{B}^{1+1}$ of local algebras is covariant with respect to the representation $U^{\mathcal{A}} \times U^{\mathcal{A}^{\prime}}$. Because of the identity $U^{\mathcal{A}}(\tilde{R}(2 \pi)) U^{\mathcal{A}^{\prime}}(\tilde{R}(2 \pi))=\mathbb{1}$ the set $\mathcal{B}^{1+1}$ is in fact labelled by the double cones in $\widetilde{\mathbb{M}}$, and $U^{\mathcal{A}} \times U^{\mathcal{A}^{\prime}}$ is a representation of the conformal group in $1+1$ dimensions, namely the group $\left(P S L(2, \mathbb{R})^{\sim} \times P S L(2, \mathbb{R})^{\sim}\right) / \mathbb{Z}$. The spectrum condition for $U^{\mathcal{A}} \times U^{\mathcal{A}^{\prime}}$ was proved in corollary II.15. 


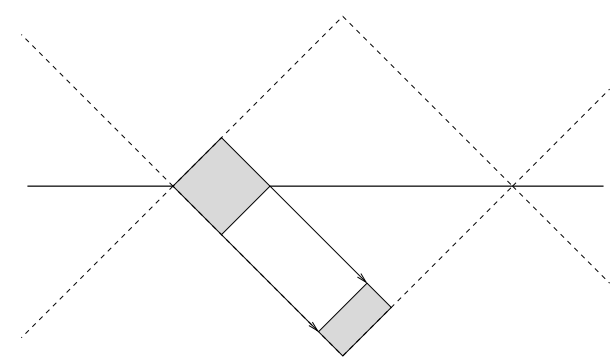

Figure IV.1: Chiral transformations and locality in $d=1+1$.

The vacuum vector is invariant with respect to $U^{\mathcal{A}} \times U^{\mathcal{A}^{\prime}}$ (corollary II.16) and it is a basis for the space of vectors with this property, because the space of $U$-invariant vectors is one-dimensional. $\Omega$ is cyclic for all local algebras in $\mathcal{B}^{1+1}$ because of the REEH-SCHLIEDER property of $\mathcal{B}$.

Isotony follows directly from the net-endomorphism property. An inclusion of 1+1-dimensional double cones $\tilde{g}_{L} I \times \tilde{g}_{R} I \subset \tilde{h}_{L} I \times \tilde{h}_{R} I$ contained in Minkowski space $\mathbb{M}$, yields the relations: $\tilde{h}_{L, R}{ }^{-1} \tilde{g}_{L, R} I \subset I$. Applying proposition IV.6 we get: $A d_{U \mathcal{A}^{\prime}\left(\tilde{h}_{R}-1 \tilde{g}_{R}\right) \cup \mathcal{A}\left(\tilde{h}_{L}^{-1} \tilde{g}_{L}\right)} \mathcal{B}(I) \subset \mathcal{B}(I)$. This is equivalent to $\mathcal{B}^{1+1}\left(\tilde{g}_{L} I \times \tilde{g}_{R} I\right) \subset$ $\mathcal{B}^{1+1}\left(\tilde{h}_{L} I \times \tilde{h}_{R} I\right)$.

Locality for double cones in $\mathbb{M}$ is shown easily as well. We can reduce the discussion to the situation where there is a double cone $J_{1} \times J_{2}$ spacelike to our basic time zero double cone $I \times I$ simply by applying an appropriate transformation. There is a time zero double cone $J \times J$ which contains $J_{1} \times J_{2}$ and is spacelike to $I \times I$. Since we have shown isotony for $\mathcal{B}^{1+1}$, locality for this set follows from locality of $\mathcal{B}$.

Weak additivity may be proved as in the chiral case. By scale covariance the local algebras of $\mathcal{B}^{1+1}$ are continuous from the inside as well as from the outside [LRT78]. Because we can restrict the discussion to time zero algebras and since the argument of Jörss [Jör96] for the corresponding chiral situation may be extended directly, we have weak additivity for $\mathcal{B}^{1+1}$.

The proof is complete, if one recognises that the proof of [BGL93, proposition 1.9], which establishes the unique extendibility of $\mathcal{B}^{1+1}$ from $\mathbb{M}$ to all of $\widetilde{\mathbb{M}}$, only requires the prerequisites established so far. In particular, not the spectrum condition itself is needed, but only its consequence, the REEH-SCHLIEDER property.

In light of this theorem we obtain a straightforward interpretation of the subgeometrical action of $U^{\mathcal{A}}$ on $\mathcal{B}$. If we apply a chiral coordinate transformation $\tilde{g}_{R}$ to a time zero double cone $J \times J$ and if we test the localisation of the correspondingly transformed local algebra of $\mathcal{B}^{1+1}$ only by looking at time zero algebras, then we find that the result commutes just with time zero algebras $\mathcal{B}(K)$ assigned to proper intervals $K$ contained in the causal complement of $\gamma_{\tilde{g}_{R}} J$. The statement of proposition IV.6 follows from HAAG duality of $\mathcal{B}$. (Compare figure IV.1.)

The theorem has some direct applications to chiral subtheories and their 
Coset models: We have found that the maximal CoseT model $\mathcal{C}_{\max }$ associated with a subtheory $\mathcal{A} \subset \mathcal{B}$ may be regarded as the chiral conformal theory of all right chiral observables in $\mathcal{B}^{1+1}$ in the sense of REHREN [Reh00], ie the local observables of $\mathcal{B}^{1+1}$ which are invariant under the action of transformations on the left light-cone coordinate only.

The observables of $\mathcal{A}$ may be viewed as left chiral observables and the chiral conformal subnet $\mathcal{A}_{\text {max }} \subset \mathcal{B}$ consisting of local observables invariant with respect to the action of $U^{\mathcal{A}^{\prime}}$ (and accordingly covariant with respect to the action of $U^{\mathcal{A}}$ ) is to be identified with the chiral theory of all left chiral observables in $\mathcal{B}^{1+1}$.

Thus, we have identified $\mathcal{A}_{\max }$ and $\mathcal{C}_{\max }$ as fixed-points of a spacetime symmetry acting on a suitably extended theory, namely $\mathcal{B}^{1+1}$. In presence of the net-endomorphism property it is not necessary to extend the "classical" symmetry concept (see eg [Ara92]), if one wants to interpret the chiral subtheories $\mathcal{A}_{\text {max }}$ and $\mathcal{C}_{\text {max }}$ as fixed-points of a symmetry; all one has to do is to extend the theory $\mathcal{B}$ to its holographic image $\mathcal{B}^{1+1}$. Generalisations of the symmetry concept are necessary for a large class of chiral conformal subtheories [LR95, Reh94b].

Another interesting, direct consequence of theorem IV.7 is the following: The cyclic subspaces of $\mathcal{C}_{\max }$ and $\mathcal{A}_{\max }$, namely $\overline{\mathcal{C}_{\max }(I) \Omega}$ and $\overline{\mathcal{A}_{\max }(I) \Omega}$, coincide with the spaces of $U^{\mathcal{A}}$ - and $U^{\mathcal{A}^{\prime}}$-invariant vectors, respectively, as was shown by REHREN [Reh00, lemma 2.3]. The proof of the following proposition includes an alternative proof of this statement; together with the other parts, this proposition may be viewed as a generalised version of [Xu00a, theorem 2.4], which applies to a particular class of chiral subnets.

Proposition IV.8: Assume $U^{\mathcal{A}}$ to have the net-endomorphism property and denote the projections onto the subspaces of $U^{\mathcal{A}}$ - and $U^{\mathcal{A}^{\prime}}$-invariant vectors by $E_{U^{\mathcal{A}}}$ and $E_{U_{\mathcal{A}^{\prime}}}$, respectively. Then we have for the maximal $U^{\mathcal{A}}$-covariant extension of $\mathcal{A}$, given by $\mathcal{A}_{\text {max }}(I)=\left\{U^{\mathcal{A}^{\prime}}\right\}^{\prime} \cap \mathcal{B}(I)$, and the maximal CosET model associated with $\mathcal{A} \subset \mathcal{B}$, given by $\mathcal{C}_{\text {max }}(I)=\left\{U^{\mathcal{A}}\right\}^{\prime} \cap \mathcal{B}(I)$, for arbitrary $I \Subset \mathrm{S}^{1}$ :

$$
\overline{\mathcal{A}_{\max }(I) \Omega}=E_{U_{\mathcal{A}^{\prime}}} \mathscr{H}, \quad \overline{\mathcal{C}_{\max }(I) \Omega}=E_{U \mathcal{A}} \mathscr{H}
$$

For any Coset model $\mathcal{C}$ associated with $\mathcal{A} \subset \mathcal{B}$ we have a unitary equivalence of chiral conformal theories: $\mathcal{A} \subseteq \mathcal{C} e_{\mathcal{A} \odot \mathcal{C}} \cong \mathcal{A} e_{\mathcal{A}} \otimes \mathcal{C} e_{\mathcal{C}} \cdot E_{U^{\mathcal{A}}} \mathscr{H}$ has a direct interpretation as multiplicity space of the vacuum subrepresentation of $\mathcal{A} \subset \mathcal{B}$.

Proof: Concerning the proof of (IV.6) it suffices to deal with the case $I=\mathrm{S}_{+}^{1}$ (because of the REEH-SCHLIEDER theorem). By lemma A.1 the spaces of vectors which are invariant with respect to translations are identical with $E_{U^{\mathcal{A}}} \mathscr{H}$ and $E_{U^{\mathcal{A}^{\prime}}} \mathscr{H}$, respectively. Taking into account corollary IV.5 above the statement (IV.6) was proved by Borchers [Bor98, theorem 2.6.3].

The statement on the tensor-product character of vacuum representations of CoseT pairs was proved for proposition II.6 already. 
In the following, $A$ denotes local observables in $\mathcal{A} \subset \mathcal{B}$ and $\pi_{0}(A)=A e_{\mathcal{A}}$ its representative in the vacuum representation on $e_{\mathcal{A}} \mathscr{H}=: \mathscr{H}_{0}$. The implementation of conformal covariance in $\pi_{0}$ shall be written $U_{0}$. For every vacuum subrepresentation in $\mathcal{A} \subset \mathcal{B}$ there is a partial isometry $R: \mathscr{H} \rightarrow \mathscr{H}_{0}$ satisfying $R A=\pi_{0}(A) R$ for all local $A$ in $\mathcal{A} \subset \mathcal{B}$.

The projection $e_{R}:=R^{*} R$ commutes with all of $\mathcal{A}$. $R U^{\mathcal{A}}(.) R^{*}$ is a unitary strongly continuous representation of $\operatorname{PSL}(2, \mathbb{R})^{\sim}$ which implements global conformal covariance in $\pi_{0}$, thus: $R U^{\mathcal{A}}(.) R^{*}=U_{0}($.$) . It follows directly that$ $\Phi_{\Omega}:=R^{*} \Omega$, the vacuum of the subrepresentation associated with $R$, is invariant with respect to $U^{\mathcal{A}}$, ie $\Phi_{\Omega} \in E_{U \mathcal{A}} \mathscr{H}$. This completes the proof of the last statement.

It is not clear in general that the representation $\mathcal{A} \subseteq \mathcal{C}_{\text {max }} \subset \mathcal{B}$ of the tensorproduct theory defined by the vacuum representation of a chiral subnet $\mathcal{A} \subset$ $\mathcal{B}$ and the vacuum representation of its maximal Coset model has a (spatial) tensor-product decomposition. This is known under certain conditions [KLM01].

Proposition IV.8 gives results from character arguments on inclusions of current algebras a direct and rigorous meaning in the context of the analysis of the respective inclusions of chiral conformal theories and CoseT models. We will discuss this in section $\mathrm{V}+3$.

\subsection{Sharp geometrical action and time-like commutativity}

It is interesting to look at two properties which are closely related to but stronger than the net-endomorphism property. The first of these, the sharp geometrical action, is sufficient for reinterpreting the holographic quasi-theory as a physically sensible model:

Definition IV.9: We say that the unique inner-implementing representation $U^{\mathcal{A}}$ of a chiral subnet $\mathcal{A} \subset \mathcal{B}$ has sharp geometrical action (on $\mathcal{B}$ ) if for every $I \Subset \mathrm{S}^{1}$ and for every $\tilde{g}$ of $\operatorname{PSL}(2, \mathbb{R})^{\sim}$ we have:

$$
A d_{U^{\mathcal{A}}(\tilde{g})} \mathcal{B}(I) \subset \mathcal{B}\left(\gamma_{\tilde{g}}(I)\right) \cap \mathcal{B}\left(\gamma_{\tilde{g}}^{\prime}(I)^{\prime}\right) .
$$

Here, we have introduced a new notation: $\gamma_{\tilde{g}}^{\prime}(I):=\gamma_{\tilde{g}}(I) \backslash(\bar{I} \cup \mathrm{p}(\tilde{g}) \bar{I})$. For the case $\gamma_{\tilde{g}}^{\prime}(I)=\varnothing$ we set $\varnothing^{\prime}:=\mathrm{S}^{1}, \mathcal{B}(\varnothing):=\mathbb{C} \mathbb{1}$ and $\mathcal{B}\left(\varnothing^{\prime}\right):=\mathcal{B}(\varnothing)^{\prime}$.

One recognises immediately that sharp geometrical action of $U^{\mathcal{A}}$ implies the same property for $U^{\mathcal{A}^{\prime}}$ :

$$
\begin{aligned}
A d_{U^{\mathcal{A}^{\prime}\left(\tilde{g}^{-1}\right)}} \mathcal{B}(I) & \subset \mathcal{B}\left(\gamma_{\tilde{g}}\left(\mathrm{p}\left(\tilde{g}^{-1}\right) I\right)\right) \cap \mathcal{B}\left(\gamma_{\tilde{g}}^{\prime}\left(\mathrm{p}\left(\tilde{g}^{-1}\right) I\right)^{\prime}\right) \\
& =\mathcal{B}\left(\gamma_{\tilde{g}^{-1}}(I)\right) \cap \mathcal{B}\left(\gamma_{\tilde{g}^{-1}}^{\prime}(I)^{\prime}\right)
\end{aligned}
$$

Here we have used the elementary identity $\gamma_{\tilde{g}}\left(\mathbf{p}\left(\tilde{g}^{-1}\right) I\right)=\gamma_{\tilde{g}^{-1}}(I)$. 


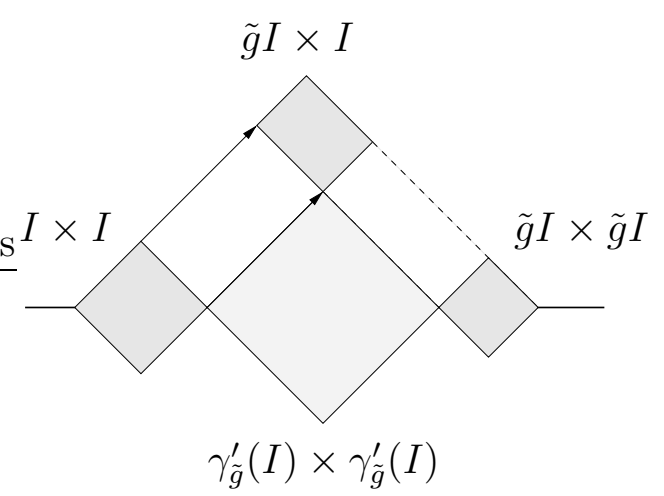

Figure IV.2: Sharp geometrical action and timelike commutativity.

For $\tilde{g}$ close to $i d$ we have $I \cup \mathrm{p}(\tilde{g}) I=\gamma_{\tilde{g}}(I) \Subset \mathrm{S}^{1}$ and the inclusion (IV.7) is clear, if we have the net-endomorphism property. In fact, sharp geometrical action implies the net-endomorphism property. When $I$ and $\mathrm{p}(\tilde{g}) I$ become disjoint, then (generically) the complement of $I$ and $\mathrm{p}(\tilde{g}) I$ in $\mathrm{S}^{1}$ consists of two proper, disjoint intervals, $\gamma_{\tilde{g}}(I)^{\prime}$ and $\gamma_{\tilde{g}}^{\prime}(I)$; sharp geometrical action says in this case, that $A d_{U^{\mathcal{A}}(\tilde{g})} \mathcal{B}(I)$ commutes with all observables associated with either localisation region.

Proposition IV.10: Assume $U^{\mathcal{A}}$ to have the net-endomorphism property. Then sharp geometrical action is equivalent to commutativity for timelike separation in the quasi-theory $\mathcal{B}^{1+1}$, which thus may be reinterpreted as a physical model.

Proof: We take a $\tilde{g} \subset P S L(2, \mathbb{R})^{\sim}$ such that $I$ and $\tilde{g} I$ both lie in a single copy of $\mathrm{S}^{1}$ in $\mathbb{R}$ and are disconnected: $\bar{I} \cap \tilde{g} \bar{I}=\varnothing$. Assuming sharp geometrical action for $U^{\mathcal{A}}$ we have:

$$
A d_{U^{\mathcal{A}}(\tilde{g})} \mathcal{B}(\mathrm{p} I)=\mathcal{B}^{1+1}(\tilde{g} I \times I) \subset \mathcal{B}\left(\mathrm{p} \gamma_{\tilde{g}}^{\prime}(I)\right)^{\prime} .
$$

Repeating the argument with $U^{\mathcal{A}^{\prime}}$, varying $I$ and $\tilde{g}$ it is clear that sharp geometrical action implies timelike commutativity of $\mathcal{B}^{1+1}$. The opposite direction is obvious. (Compare figure IV.2).

Hence, in presence of sharp geometrical action the distinction between timelike and spacelike directions from locality disappears. We may swap the time axis with the space axis. Then the original chiral local algebras become $x$-zero algebras associated with intervals on the time axis. With this definition the usual spectrum condition holds.

Making some assumptions on the inclusion $\mathcal{A}_{\max }\left(\mathcal{C}_{\max } \subset \mathcal{B}\right.$ we give a straightforward analysis in chapter $\mathrm{V}$ which yields a necessary condition on a possible sharp geometrical action: the spectrum of $U^{\mathcal{A}}(\tilde{R}(2 \pi))$ has to be contained in $\{ \pm 1\}$. This condition excludes sharp geometrical action for all current subalgebras known to the author, except the ones one can make up trivially; section $\mathrm{V}, 3$ contains some examples of current subalgebras. 
A very special sub-geometrical action arises, if $\mathcal{B}$ is actually generated by a Coset pair $\mathcal{A}_{\max } \subseteq \mathcal{C}_{\max }$, ie we have $e_{\mathcal{A}_{\max } \odot \mathcal{C}_{\max }}=\mathbb{1}$. This we call a conormal chiral subnet $\mathcal{A}_{\max } \subset \mathcal{B}$. In this case, obviously, we have a stronger version of sharp geometrical action which we shall call completely sharp geometrical action:

$$
A d_{U^{\mathcal{A}}(\tilde{g})} \mathcal{B}(I) \subset \mathcal{B}(I) \vee \mathcal{B}(\mathrm{p}(\tilde{g}) I) .
$$

It is not known, if or under which additional conditions sharp geometrical action actually implies conormality of the subnet $\mathcal{A}_{\max } \subset \mathcal{B}$ and thus the character of $\mathcal{B}$ as a tensor product of chiral conformal theories. But we have some immediate remarks to make.

Obviously, completely sharp geometrical action implies sharp geometrical action. Both are equivalent if for every pair of proper intervals $I_{0}, I_{2}$ for which $\mathrm{S}^{1} \backslash \overline{I_{0}} \cup \overline{I_{2}}$ is the union of two disjoint proper intervals $I_{1}, I_{3}$ the inclusion

$$
\mathcal{B}\left(I_{0}\right) \vee \mathcal{B}\left(I_{2}\right) \subset\left(\mathcal{B}\left(I_{1}\right) \vee \mathcal{B}\left(I_{3}\right)\right)^{\prime}
$$

is actually an equality. Generically, the inclusion is non-trivial and for completely rational $\mathcal{B}$ equality implies that all locally normal representations of $\mathcal{B}$ are unitarily equivalent to its vacuum representation [KLM01]. This is seldom the case; however, $\mathcal{L} E(8)_{1}$ is an example of this, cf section V 3.3 . Inclusions of the type (IV.10) have been studied eg in [Sta95, Xu00b, KLM01]. The index of the inclusion in (IV.10) is the $\mu$-index of $\mathcal{B}$ [KLM01] (cf chapter V).

Sharp geometrical action is weaker than conormality of $\mathcal{A}_{\max } \subset \mathcal{B}$. The $1+1$ dimensional conformal theory defined by tensor products of free, massless, chiral fermions $\psi_{L, R}$ (bounded Bose fields, cf [Bau97, Bau99, GR00, Reh97]) fulfills timelike commutativity, but is manifestly not generated by the subtheories defined by its maximal chiral observables, $\mathcal{A}_{L, R}^{\max }$, in the sense of REHREn [Reh00]. Since the chiral fermion fields possess a stress-energy tensor, this model may be reinterpreted in the sense of chiral subnets and their CosET models: we restrict it to the time axis and look at the subnets induced by the inclusions of the maximal chiral observables in the whole theory. This gives subnets with sharp geometrical action of the respective inner-implementing representations, but the inclusions are not conormal. It is doubtful, whether the restriction to $x$-zero algebras does yield a subnet $\mathcal{A}_{L}^{\max } \subset \mathcal{B}$ with a completely sharp geometrical action for $U^{\mathcal{A}_{L}^{\max }}$, as products $\psi_{L}(f) \otimes \psi_{R}(g), \operatorname{supp}(f) \subset I, \operatorname{supp}(g) \subset J$ do not seem to be approximated by operators from $\mathcal{B}(I \times I)$ and $\mathcal{B}(J \times J)$.

It is clear that the adjoint action of $U^{\mathcal{A}}(\tilde{R}(2 \pi))$ induces an automorphism of each local algebra $\mathcal{B}(I), I \Subset \mathrm{S}^{1}$, if $U^{\mathcal{A}}$ has sharp geometrical action (see definition IV.9). This improves our knowledge on the net-endomorphic character of the action of $U^{\mathcal{A}}$ on $\mathcal{B}$ (proposition IV.6), but it does not apply to many subnets. A more relevant statement of the same type can be verified for a broad class of Coset pairs (proposition V.11). 


\section{Solving the isotony problem}

In this section we use the AdDitional Assumption to solve the isotony problem for the local relative commutants $\mathcal{C}_{I}$ of an inclusion of chiral conformal theories, $\mathcal{A} \subset \mathcal{B}$. Once their isotony is proved, they are known to coincide with the local algebras of the maximal Coset model $\mathcal{C}_{\text {max }}$ associated with $\mathcal{A} \subset \mathcal{B}$. This way, we reach one of the main goals of this work: the maximal COSET model is found to be of a local nature, ie it is determined completely by local data.

The isotony problem is solved in two steps: A crucial, purely group theoretical lemma (lemma A.1) admits a simple, accessible characterisation of the isotony problem, which we give in proposition IV.11. Aside of being an intermediate step of our analysis, it illustrates the character of the isotony problem. The argument is completed by an application of the AdDitional Assumption (lemma IV.12) and summarised in the main theorem of this work (theorem IV.13).

Proposition IV.11: Assume the unique inner-implementing representation $U^{\mathcal{A}}$ associated with a chiral subnet $\mathcal{A} \subset \mathcal{B}$ to have the net-endomorphism property. Referring to $I \Subset \mathrm{S}^{1}$, $e_{I}^{c}$ shall denote the projection onto the HILBERT subspace which the local relative commutant $\mathcal{C}_{I}=\mathcal{A}(I)^{\prime} \cap \mathcal{B}(I)$ generates from the vacuum. The following are equivalent:

i) For some pair $I, K$ of intervals satisfying $K \subsetneq I \Subset \mathrm{S}^{1}$ holds: $e_{K}^{c} \subset e_{I}^{c}$.

ii) $\mathcal{C}_{\mathrm{S}_{+}^{1}} \subset\left\{U^{\mathcal{A}}(\tilde{D}(t)), t \in \mathbb{R}\right\}^{\prime}$.

iii) $\mathcal{C}_{\max }(I)=\left\{U^{\mathcal{A}}(\tilde{g}), \tilde{g} \in P S L(2, \mathbb{R})^{\sim}\right\}^{\prime} \cap \mathcal{B}(I)=\mathcal{C}_{I}, I \Subset \mathrm{S}^{1}$.

Remark: The statement on the cyclic projections is non-trivial since, although the local relative commutants are manifestly covariant with respect to $U$, the REEH-SCHLIEDER theorem does not apply due to the unclear status of isotony (cf eg [Bor68]).

Proof: The implications iii) $\Rightarrow$ i), ii) are obvious. We start the proof proper with a discussion on $\mathrm{i}$ ) $\Rightarrow$ iii) and here we look at the case $I=\mathrm{S}_{+}^{1}$ (general case by covariance). We set $e_{\mathrm{S}_{+}^{1}}^{c}=e_{+}^{c}$. The inclusion $e_{K}^{c} \subset e_{+}^{c}$ yields by the separating property of the vacuum and modular covariance of $\mathcal{C}_{\mathrm{S}_{+}^{1}} \subset \mathcal{B}\left(\mathrm{S}_{+}^{1}\right): \mathcal{C}_{K} \subset \mathcal{C}_{\mathrm{S}_{+}^{1}}$. Thus, any $g \in P S L(2, \mathbb{R})$ satisfying $g \mathrm{~S}_{+}^{1}=K$ leads to an operator $U(g)$ which leaves $e_{+}^{c} \mathscr{H}$ globally invariant. $g$ has the form $g=S(n) T(s) D(t), n, s \geq 0 . g$ may be chosen such that $t=0$.

By modular covariance $J$, the modular conjugation of $\mathcal{B}\left(\mathrm{S}_{+}^{1}\right)$, and $e_{+}^{c}$ commute and, by covariance and the Bisognano-WICHMAnn property of $\mathcal{B}, A_{J U(R(\pi))}$ induces an automorphism of $\mathcal{C}_{\mathrm{S}_{+}^{1}}$, so $e_{+}^{c}$ commutes with $U(R(\pi))$, too. The relations $J T(s) J=T(-s), J S(n) J=S(-n)$ lead to $U(S(-n)) U(T(-s)) e_{+}^{c} \mathscr{H} \subset e_{+}^{c} \mathscr{H}$. We assume $n, s>0$ and define

$$
g(n, s):=S\left(-n \frac{n s+(1+n s)^{2}}{2+n s}\right) T\left(-s \frac{2+n s}{n s+(1+n s)^{2}}\right)(S(n) T(s))^{2} .
$$


Applying scale covariance we arrive at: $U(g(n, s)) e_{+}^{c} \mathscr{H} \subset e_{+}^{c} \mathscr{H}$. The group element $g(n, s)$ leaves the point $1 \in \mathrm{S}^{1}$ invariant and is not a pure scale transformation. This proves that all special conformal transformations leave $e_{c}^{+}$invariant. The same follows for the translations because of $R(\pi) S(n) R(\pi)=T(-n)$, which proves $\left[U(g), e_{+}^{c}\right]=0$ for all $g \in P S L(2, \mathbb{R})$ recognising that translations and special conformal transformations generate the whole group (see section II.2). For $n=0$ or $s=0$ the last part applies directly. This proves: $e_{K}^{c}=e_{+}^{c}$ for all $K \Subset \mathrm{S}^{1}$. By modular covariance of the inclusions $\mathcal{C}_{K} \subset \mathcal{B}(K)$ we have $\mathcal{C}_{K}=\left\{e_{K}^{c}\right\}^{\prime} \cap \mathcal{B}(K)$ and this yields isotony for the local relative commutants. The remainder follows by maximality of $\mathcal{C}_{\max }$ (lemma II.4).

Finally we discuss the implication ii) $\Rightarrow$ iii). If $B \in \mathcal{B}\left(\mathrm{S}_{+}^{1}\right)$ commutes with $U^{\mathcal{A}}(\tilde{D}(t)), t \in \mathbb{R}$, then $B \Omega$ is invariant under the action of all of $U^{\mathcal{A}}$ (lemma A.1). If $\tilde{g}$ is sufficiently close to the identity, $A d_{U^{\mathcal{A}}(\tilde{g})}(B)$ is a local operator (proposition IV.6), and the separating property of the vacuum proves that $B$ commutes with all of $U^{\mathcal{A}}$. Thereby, we arrive at $\mathcal{C}_{\mathrm{S}_{+}^{1}} \subset \mathcal{C}_{\max }\left(\mathrm{S}_{+}^{1}\right)$, provided the assumption in ii) holds. The other inclusion is trivial.

Remark: The dilatations $U^{\mathcal{A}}(\tilde{D}(t)), t \in \mathbb{R}$, induce automorphisms of $\mathcal{B}\left(\mathrm{S}_{+}^{1}\right)$ and the last part of the proof shows $\mathcal{C}_{\max }\left(\mathrm{S}_{+}^{1}\right)$ to be the fixed-point subalgebra with respect to this automorphism group. Covariance leads to a corresponding identification of every $\mathcal{C}_{\max }(I), I \Subset \mathrm{S}^{1}$. This may be regarded as an alternative "local" characterisation of $\mathcal{C}_{\text {max }}$, but since the automorphism groups are determined by global observables, namely non-trivial unitaries from $U^{\mathcal{A}}$, this is not satisfactory.

Only for the final step of our analysis we need to invoke the AdDitional Assumption once again:

Lemma IV.12: Assume the Additional Assumption to hold. Then we have: $U^{\mathcal{A}}(\tilde{D}(t)) \in \mathcal{A}\left(\mathrm{S}_{+}^{1}\right) \vee \mathcal{A}\left(\mathrm{S}_{-}^{1}\right), t \in \mathbb{R}$, and $U^{\mathcal{A}}$ has the net-endomorphism property.

Proof: According to the Additional Assumption and lemma A.4 there exist, for small, fixed $t$, diffeomorphisms $g_{\delta}, g_{\varepsilon}$ localised in arbitrarily small neighbourhoods of $+1 \in \mathrm{S}^{1}$ and $-1 \in \mathrm{S}^{1}$, respectively, and diffeomorphisms $g_{+}^{\tau_{1}, \tau_{2}}, g_{-}^{\tau_{1}, \tau_{2}}$ which are localised in $\mathbf{S}_{+}^{1}$ and $\mathbf{S}_{-}^{1}$, respectively, and phases $\varphi\left(\tau_{1}, \tau_{2}\right)$ such that for $\tau_{1,2} \in \mathbb{R}_{+}$:

$$
\begin{aligned}
U^{\mathcal{A}}(\tilde{D}(t))= & \varphi\left(\tau_{1}, \tau_{2}\right) \Upsilon^{\mathcal{A}}\left(\mathrm{p}^{-1}\left(g_{+}^{\tau_{1}, \tau_{2}}\right)\right) \Upsilon^{\mathcal{A}}\left(\mathrm{p}^{-1}\left(g_{-}^{\tau_{1}, \tau_{2}}\right)\right) \\
& \cdot A d_{U^{\mathcal{A}}\left(\tilde{D}\left(\tau_{1}\right)\right)}\left(\Upsilon^{\mathcal{A}}\left(\mathrm{p}^{-1}\left(g_{\varepsilon}\right)\right)\right) A d_{U^{\mathcal{A}}\left(\tilde{D}\left(-\tau_{2}\right)\right)}\left(\Upsilon^{\mathcal{A}}\left(\mathrm{p}^{-1}\left(g_{\delta}\right)\right)\right) .
\end{aligned}
$$

Following RoBerTs [Rob74, corollary 2.5], dilatation invariance of the vacuum and the shrinking supports ensure that the last two operators converge weakly to their vacuum expectation values in the limit $\tau_{1,2} \rightarrow \infty$. We rewrite the equation above:

$$
A d_{U^{\mathcal{A}}\left(\tilde{D}\left(\tau_{1}\right)\right)}\left(\Upsilon^{\mathcal{A}}\left(\mathrm{p}^{-1}\left(g_{\varepsilon}\right)\right)\right) A d_{U^{\mathcal{A}}\left(\tilde{D}\left(-\tau_{2}\right)\right)}\left(\Upsilon^{\mathcal{A}}\left(\mathrm{p}^{-1}\left(g_{\delta}\right)\right)\right) U^{\mathcal{A}}(\tilde{D}(t))^{*}
$$




$$
=\overline{\varphi\left(\tau_{1}, \tau_{2}\right)} \Upsilon^{\mathcal{A}}\left(\mathrm{p}^{-1}\left(g_{+}^{\tau_{1}, \tau_{2}}\right)\right)^{*} \Upsilon^{\mathcal{A}}\left(\mathrm{p}^{-1}\left(g_{-}^{\tau_{1}, \tau_{2}}\right)\right)^{*}
$$

The operators to the right converge weakly by this equation in the limit $\tau_{1}, \tau_{2} \rightarrow$ $\infty$. For small $t, g_{\varepsilon}$ and $g_{\delta}$ may be chosen close to the identity, $\omega($.$) is continuous$ and normalised, which means that for $g_{\varepsilon}, g_{\delta} \approx i d$ we have $\omega\left(\Upsilon^{\mathcal{A}}\left(\mathrm{p}^{-1}\left(g_{\varepsilon}\right)\right)\right) \neq 0$, $\omega\left(\Upsilon^{\mathcal{A}}\left(\mathrm{p}^{-1}\left(g_{\delta}\right)\right)\right) \neq 0$. This implies $U^{\mathcal{A}}(\tilde{D}(t)) \in \mathcal{A}\left(\mathrm{S}_{+}^{1}\right) \vee \mathcal{A}\left(\mathrm{S}_{-}^{1}\right)$ for small and hence for all $t$.

Because $\Upsilon^{\mathcal{A}}\left(\mathrm{p}^{-1}\left(g_{+}^{\tau_{1}, \tau_{2}}\right)\right)$ and $\Upsilon^{\mathcal{A}}\left(\mathrm{p}^{-1}\left(g_{-}^{\tau_{1}, \tau_{2}}\right)\right)$ are unitary operators, the righthand side of equation (IV.11) converges, up to a phase, strongly against $U^{\mathcal{A}}(\tilde{D}(t))$ for small $t$. This strong convergence proves that for $B \in \mathcal{B}\left(\mathrm{S}_{+}^{1}\right)$ and small $t$ holds true in the weak topology:

$$
U^{\mathcal{A}}(\tilde{D}(t)) B U^{\mathcal{A}}(\tilde{D}(t))^{*}=\lim _{\tau_{1}, \tau_{2} \rightarrow \infty} A d_{\Upsilon \mathcal{A}\left(\mathbf{p}^{-1}\left(g_{+}^{\tau_{1}, \tau_{2}}\right)\right)}(B) \in \mathcal{B}\left(\mathrm{S}_{+}^{1}\right)
$$

This establishes the net-endomorphism property (definition IV.1).

The first statement of this lemma holds trivially, if the global algebra $\mathcal{A}$ coincides with $\mathcal{A}\left(\mathrm{S}_{+}^{1}\right) \vee \mathcal{A}\left(\mathrm{S}_{-}^{1}\right)$. This is a desirable property, eg for the Connes' fusion approach to superselection structure (cf eg [Was98]), and it holds true in presence of strong additivity, but a proof of it relying on general properties of chiral conformal subtheories seems out of reach.

However, it seems natural for the representatives $U^{\mathcal{A}}(\tilde{D}(t)), t \in \mathbb{R}$, to be contained in $\mathcal{A}\left(\mathrm{S}_{+}^{1}\right) \vee \mathcal{A}\left(\mathrm{S}_{-}^{1}\right)$. One may compare this with the construction of the inner implementation of translations in $\bigvee_{I \subseteq \mathbb{R}} \mathcal{A}(I)$ using the spectrum condition [Bor66]. Here, the translations operate as geometrical automorphism group on the net of local V.NEUMANN algebras associated with localisation regions which do not contain the fixed-point of translations and they are implementable by a unitary group contained in the V.NEUMANN algebra which is generated by this net. The action of $U^{\mathcal{A}}(\tilde{D}()$.$) on \mathcal{A}\left(\mathrm{S}_{+}^{1}\right) \vee \mathcal{A}\left(\mathrm{S}_{-}^{1}\right)$ has the same character in this respect.

The proof of lemma IV.12 relies on the structure of Diff $\left(\mathrm{S}^{1}\right)$ in order to establish convergence by equation (IV.11). This procedure does not apply to the representation of dilatations through products of an exponentiated smeared stress-energy tensor as in equation (A.3). If one seeks for nets of test functions $f_{\delta}, f_{\varepsilon}$ whose supports shrink to the points +1 and -1 , respectively, while the properties needed for establishing equation (A.3) are upheld, one lacks control over the vacuum expectation values of $\exp \left(-i t \Theta^{\mathcal{A}}\left(f_{\varepsilon}\right)^{-}\right), \exp \left(-i t \Theta^{\mathcal{A}}\left(f_{\delta}\right)^{-}\right)$. Such control could allow to establish non-zero weak limit points for this procedure ${ }^{5}$ and thus to give an alternative proof for lemma IV.12 doing without the structure of $\operatorname{Diff}_{+}\left(\mathrm{S}^{1}\right)$.

We summarise and state the main result of this work, which proves that the maximal Coset models are of a local nature:

\footnotetext{
${ }^{5}$ Compare discussion in [BSM90, section 4] on strong additivity of the $U(1)$-current algebra.
} 
Theorem IV.13: Let $\mathcal{A} \subset \mathcal{B}$ be a chiral conformal subtheory and suppose the AdDitional Assumption to hold. Then the unique inner-implementing representation $U^{\mathcal{A}}$ has the net-endomorphism property and for all $I \Subset \mathrm{S}^{1}$ there holds:

$$
\mathcal{C}_{\text {max }}(I)=\left\{U^{\mathcal{A}}(\tilde{g}), \tilde{g} \in P S L(2, \mathbb{R})^{\sim}\right\}^{\prime} \cap \mathcal{B}(I)=\mathcal{A}(I)^{\prime} \cap \mathcal{B}(I)=\mathcal{C}_{I} .
$$

Proof: The net-endomorphism property of $U^{\mathcal{A}}$ holds by lemma IV.2 (and lemma IV.12), and ii) in proposition IV.11 is fulfilled because of lemma IV.12.

In the cases where both $\mathcal{A}$ and $\mathcal{B}$ possess an integrable stress-energy tensor, and hence $\mathcal{C}_{\text {max }}$ alike, the theorem means in particular: $\mathcal{A}_{\max }(I)$ and $\mathcal{C}_{\text {max }}(I)$, $I \Subset \mathrm{S}^{1}$, are their mutual relative commutants in $\mathcal{B}(I)$. We have learned that the Coset pair $\mathcal{A}_{\max } \subseteq \mathcal{C}_{\max } \subset \mathcal{B}$ is a typical object for studies on the structures related to chiral subnets an their CosET models. For these typical CosET pairs we introduce a new term reflecting the absence of the isotony problem both for the subnet and for the COSET model:

Definition IV.14: $A$ Coset pair $\mathcal{A} \subseteq \mathcal{C} \subset \mathcal{B}$ is called normal if for all $I \Subset \mathrm{S}^{1}$ the algebras $\mathcal{A}(I)$ and $\mathcal{C}(I)$ are their mutual relative commutants in $\mathcal{B}(I)$.

For normal Coset pairs $\mathcal{A} \subseteq \mathcal{C} \subset \mathcal{B}$ the local inclusions are automatically irreducible, ie the relative commutant of $\mathcal{A} \subseteq \mathcal{C}(I)$ in $\mathcal{B}(I)$ is $\mathbb{C} \mathbb{1}$, because the local algebras $\mathcal{A}(I)$ are factors.

The main theorem proves the conclusions of REHREN [Reh00] to hold true which rely on the generating property of nets of chiral observables, if the $1+1$ dimensional theory contains a stress-energy tensor in the sense of the LüsCHERMACK theorem [FST89]. Since such a stress-energy tensor factorises into its independent chiral components, our analysis applies directly. The generating property introduced in [Reh00] resisted attempts of proof even in presence of a stress-energy tensor, unfortunately. 


\section{Chapter V}

\section{Coset pairs of finite Index}

In this chapter we want to make contact with the frameworks of nets of subfactors [LR95] and of normal canonical tensor product subfactors [Reh00]. The characteristic assumption for this purpose will be that the inclusion $\mathcal{A} \subset \mathcal{C} \subset \mathcal{B}$ is of finite index; this property is discussed in the first section, and some simple statements on the local inclusions $\mathcal{A} \subset \mathcal{C}(I) \subset \mathcal{B}(I)$ are derived. We fix some notation on the way.

In the second section, we look at the net of inclusions in the 1+1-dimensional quasi-theory $\mathcal{B}^{1+1}$ and derive their character as covariant, localisable representations of $\mathcal{A} \otimes \mathcal{C}$ along the lines of [Lon01], where the corresponding situation for chiral subnets is discussed. We exploit well-known structures of the theory of superselection sectors in low dimensions [FRS89, FRS92] in order to improve our knowledge of the net-endomorphic action of $U^{\mathcal{A}}$ and we determine the possible spectrum of $U^{\mathcal{A}}(\tilde{R}(2 \pi))$ in case $U^{\mathcal{A}}$ has sharp geometrical action.

In the third section we discuss some examples of COSET pairs, illustrating the structures developed more abstractly in the previous chapters and sections, and we interpret results of studies on current subalgebras [GKO86, KW88, KNS88] such that we get examples of normal canonical tensor product subfactors which are Coset pairs.

\section{On local inclusions of Coset pairs}

As discussed in section II 1.3 finiteness of index is presumably not a generic feature of chiral subnets. Nevertheless, there are rich structures associated with inclusions of finite index, and the theory on these is advanced and promises to teach us something regarding the general situation as well. In particular, a large number of current subalgebras $\mathcal{A} \subset \mathcal{B}$ are known to be cofinite, ie the Coset pair $\mathcal{A} \subseteq \mathcal{C}_{\max } \subset \mathcal{B}$ is known to have finite index, and hence the methods related to finite index inclusions yield interesting results on COSET models.

While there was not much need to introduce the machinery on modular co- 
variant subalgebras to the full in the previous chapters, we now have to give the related structures in more detail. Rather than duplicating existing summaries, we just state the relevant facts in a form needed here and fix our notation on the way. The general reference on what is to come is the seminal work of LONGO AND REHREN on nets of subfactors [LR95]. In this section we will be concerned with local inclusions of Coset pairs, $\mathcal{A} \subset \mathcal{C}(I) \subset \mathcal{B}(I)$.

Let $\mathcal{N} \subset \mathcal{M}$ be an inclusion of v.NEUMANN algebras with common unit $\mathbb{1}$, $\Omega$ a cyclic and separating vector for $\mathcal{M}$. $\mathcal{N}$ is a modular covariant subalgebra of $\mathcal{M}$, if it is globally invariant with respect to the modular group $\sigma$ of $(\mathcal{M}, \Omega)$ :

$$
\sigma_{t}(\mathcal{N})=\Delta_{\mathcal{M}}^{i t} \mathcal{N} \Delta_{\mathcal{M}}^{-i t}=\mathcal{N}, \quad \forall t \in \mathbb{R} .
$$

$\Delta_{\mathcal{M}}$ stands for the modular operator of $(\mathcal{M}, \Omega)$; the corresponding modular conjugation will be denoted $J_{\mathcal{M}}$. The assumption of conformal covariance of chiral subnets in definition II.2 implies that the local subalgebras are modular covariant because of the BISOGNANO-WICHMANn property of $\mathcal{B}$.

Modular covariance has remarkable consequences, mostly due to a theorem of TAKESAKI [Tak72] which states that there is a normal, faithful conditional expectation $\mu: \mathcal{M} \rightarrow \mathcal{N}$ leaving invariant the state $\omega()=.\langle\Omega, . \Omega\rangle$, ie $\omega \circ \mu=\omega$. The latter property determines $\mu$ uniquely (see below vi) on page 84).

There are equivalent ways to define conditional expectations on V.NEUMANN algebras (see eg [Str81, chapter II, §9]). We prefer to state one definition with a list of useful properties of conditional expectations taken from [KK92]. A conditional expectation $\mu: \mathcal{M} \rightarrow \mathcal{N}$ is a projection from $\mathcal{M}$ onto $\mathcal{N}$ of norm 1 , ie a linear mapping satisfying $\mu^{2}=\mu$ and $\|\mu\|=1$. Such $\mu$ has the following properties:

i) Idempotency: $\mu^{2}=\mu$,

ii) Bimodule property: $\mu\left(n_{1} m n_{2}\right)=n_{1} \mu(m) n_{2}, m \in \mathcal{M}, n_{1}, n_{2} \in \mathcal{N}$,

iii) Positivity: $\mu\left(m^{*} m\right) \geq 0, m \in \mathcal{M}$,

iv) *-property: $\mu(m)^{*}=\mu\left(m^{*}\right), m \in \mathcal{M}$,

v) Normalisation: $\mu(\mathbb{1})=\mathbb{1}$,

vi) SCHWARZ property: $\mu\left(m^{*}\right) \mu(m) \leq \mu\left(m^{*} m\right), m \in \mathcal{M}$.

$\mu$ is called faithful if $\mu\left(m^{*} m\right)=0$ is possible for $m=0$ only. $\mu$ is a normal conditional expectation if it is normal as a linear map.

Examples of conditional expectations are group means for fixed-point inclusions with respect to a finite or compact automorphism group. If the innerimplementing representation $U^{\mathcal{A}}$ associated with a chiral subnet $\mathcal{A} \subset \mathcal{B}$ has the net-endomorphism property, a result of FRIGERIO [Fri78] gives explicit formulae 
for the faithful, normal conditional expectations of the inclusions $\mathcal{A}_{\max }(I) \subset \mathcal{B}(I)$, $\mathcal{C}_{\text {max }}(I) \subset \mathcal{B}(I)$ which leave invariant the vacuum. We state the corresponding formula for the inclusion $\mathcal{C}_{\max }\left(\mathrm{S}_{+}^{1}\right) \subset \mathcal{B}\left(\mathrm{S}_{+}^{1}\right)$ :

$$
\mu_{\mathcal{C}_{\text {max }}\left(\mathrm{S}_{+}^{1}\right) \subset \mathcal{B}\left(\mathrm{S}_{+}^{1}\right)}(b)=w^{*}-\lim _{\lambda \searrow 0} \lambda \int_{0}^{\infty} d t e^{-\lambda t} A d_{U^{\mathcal{A}}(T(t))}(b), \quad b \in \mathcal{B}\left(\mathrm{S}_{+}^{1}\right) .
$$

One may use the action of dilatations in a similar manner (mean ergodic theorem, cf eg [Lan76, Pet83]).

Normal, faithful conditional expectations $\mu: \mathcal{M} \rightarrow \mathcal{N}$ which leave the state $\omega$ invariant have remarkable properties (see eg [KK92], [Bor97a, theorem 6.4], [Bor00, lemma VI.1.2]). We assume in addition $\mathcal{M}, \mathcal{N}$ to be factors and $\mathscr{H}$ to be separable. If we denote the projection onto $\mathscr{H}_{\mathcal{N}}=\overline{\mathcal{N} \Omega}$ by $e_{\mathcal{N}}$, then we have:

i) $e_{\mathcal{N}}$ commutes with $\Delta_{\mathcal{M}}^{i t}, t \in \mathbb{R}$, and $J_{\mathcal{M}}$.

ii) $\Delta_{\mathcal{M}} e_{\mathcal{N}}$ and $J_{\mathcal{M}} e_{\mathcal{N}}$ are the modular data of $\mathcal{N} e_{\mathcal{N}}$ on $\mathscr{H}_{\mathcal{N}}$.

iii) We have $e_{\mathcal{N}} \mathcal{N}^{\prime} e_{\mathcal{N}}=J_{\mathcal{M}} \mathcal{N} J_{\mathcal{M}} e_{\mathcal{N}}$.

iv) It holds true: $\mathcal{N}=\left\{e_{\mathcal{N}}\right\}^{\prime} \cap \mathcal{M}$.

v) The map $\mathcal{N} \ni n \mapsto n e_{\mathcal{N}}$ defines an isomorphism of v.NEUMANN algebras. We have $\|n\|=\left\|n e_{\mathcal{N}}\right\|$.

vi) For given $m \in \mathcal{M}$, the unique solution of the equation $e_{\mathcal{N}} m e_{\mathcal{N}}=n e_{\mathcal{N}}$, $n \in \mathcal{N}$, is $n=\mu(m)$.

The index of a normal, faithful conditional expectation $\mu: \mathcal{M} \rightarrow \mathcal{N}, \mathcal{M}, \mathcal{N}$ infinite-dimensional factors, is defined as follows :

$$
I_{\mu}:=\inf \left\{\lambda: \lambda \geqslant 1, \mu\left(m^{*} m\right) \geq \lambda^{-1} m^{*} m \forall m \in \mathcal{M}\right\} .
$$

If $I_{\mu}<\infty$ for some $\mu$, then there is a unique normal, faithful conditional expectation $\mu_{0}: \mathcal{M} \rightarrow \mathcal{N}$ which has a minimal index $I_{\mu_{0}}$. This is used to define the index of the subfactor $\mathcal{N} \subset \mathcal{M}$ :

$$
[\mathcal{M}: \mathcal{N}]:=I_{\mu_{0}}=\inf _{\mu} I_{\mu}
$$

If $\mathcal{N} \subset \mathcal{M}$ is irreducible, ie the relative commutant $\mathcal{N}^{c}:=\mathcal{N}^{\prime} \cap \mathcal{M}$ of $\mathcal{N}$ in $\mathcal{M}$ is trivial, $\mathcal{N}^{c}=\mathbb{C} \mathbb{1}$, then there is at most one normal, faithful conditional expectation $\mu: \mathcal{M} \rightarrow \mathcal{N}$ [Con73, theorem 1.5.5]. Local inclusions of chiral subnets of finite index are irreducible, as we shall see below (lemma V.1), and for this reason their respective unique normal, faithful conditional expectation is automatically minimal.

Central for the studies on (nets of) subfactors is the dual canonical endomorphism $\rho: \mathcal{N} \rightarrow \mathcal{N}$. If $\mathcal{N}$ and $\mathcal{M}$ are both properly infinite V. NEUMANN algebras 
on a separable HiLBERT space, there are vectors $\phi$ which are cyclic and separating for both $\mathcal{N}$ and $\mathcal{M}$ [DL84]. Taking the modular conjugations $J_{(\mathcal{N}, \phi)}$ and $J_{(\mathcal{M}, \phi)}$ corresponding to the pairs $(\mathcal{N}, \phi)$ and $(\mathcal{M}, \phi)$, respectively, one defines the canonical endomorphism $\gamma: \mathcal{M} \rightarrow \mathcal{N} \subset \mathcal{M}$ by:

$$
\gamma(m):=J_{(\mathcal{N}, \phi)} J_{(\mathcal{M}, \phi)} m J_{(\mathcal{M}, \phi)} J_{(\mathcal{N}, \phi)}, m \in \mathcal{M} .
$$

$\gamma$ depends only through a unitary conjugation in $\mathcal{N}$ on the choice of $\phi$ [Lon87]. The restriction of $\gamma$ to $\mathcal{N}$ defines the dual canonical endomorphism $\rho$. For inclusions of infinite factors $\mathcal{N} \subset \mathcal{M}$ with finite index $[\mathcal{M}: \mathcal{N}]$ one defines the dimensions $d_{\rho}, d_{\gamma}$ of $\rho, \gamma$ to be:

$$
d_{\rho}:=[\mathcal{N}: \rho(\mathcal{N})]^{\frac{1}{2}}, d_{\gamma}:=[\mathcal{M}: \gamma(\mathcal{M})]^{\frac{1}{2}} .
$$

We have: $d_{\rho}=d_{\gamma}=[\mathcal{M}: \mathcal{N}]$.

Regarding a chiral subnet $\mathcal{A} \subset \mathcal{B}$ one finds that the dual canonical endomorphism $\rho_{I}: \mathcal{A}(I) \rightarrow \rho_{I}(\mathcal{A}(I))$, taken as an endomorphism of the isomorphic algebra $\mathcal{A}(I) e_{\mathcal{A}}$, may be extended to a DHR endomorphism $\rho$ of $\mathcal{A} e_{\mathcal{A}}$ localised in I. The DHR endomorphism $\rho$ induces a localised representation of $\mathcal{A} e_{\mathcal{A}}$ which is unitarily equivalent to the representation of $\mathcal{A} e_{\mathcal{A}}$ by the embedding $\mathcal{A} \subset \mathcal{B}$ and denoted $\rho$ as well. This is shown in [Lon01] (mostly relying on results in [LR95]) and we will give corresponding arguments in $1+1$ dimensions in the next section.

The dimension of the representation $\rho$ is defined as:

$$
d(\rho):=\left[\rho_{I^{\prime}}\left(\mathcal{A}\left(I^{\prime}\right) e_{\mathcal{A}}\right)^{\prime}: \rho_{I}\left(\mathcal{A}(I) e_{\mathcal{A}}\right)\right]^{\frac{1}{2}}=\left[\mathcal{A}(I): \rho_{I}(\mathcal{A}(I))\right]^{\frac{1}{2}}=d_{\rho_{I}} .
$$

By the index-statistics theorem [GL96] this dimension coincides with the statistical dimension of the DHR endomorphism $\rho$. The index $[\mathcal{B}(I): \mathcal{A}(I)]$ is found to be constant for all $I \Subset S^{1}$ [Lon01]. The same arguments apply, with minor alterations, to the $1+1$-dimensional situation and we refrain from giving a proof that the index is constant in this setting as well.

The following lemma is a preparation for a simple statement on CosET pairs of finite index (proposition V.2); it shows in particular that a chiral subnet $\mathcal{A} \subset \mathcal{B}$ with finite index is a conformal inclusion in the sense of definition II.21.

Lemma V.1: Assume $\mathcal{A} \subset \mathcal{C} \subset \mathcal{B}$ to be a COSET pair of finite index. Then the local inclusions $\mathcal{A} \subset \mathcal{C}(I) \subset \mathcal{B}(I), I \Subset \mathrm{S}^{1}$, are irreducible, as are the inclusions $\mathcal{A}(I) \subset \mathcal{A}(I)^{c c}, \mathcal{C}(I) \subset \mathcal{A}(I)^{c}$.

Proof: Using formulae on the indices of a sequence of inclusions and for tensor products of such we have [Lon90, Lon89]:

$$
\begin{aligned}
{[\mathcal{B}(I): \mathcal{A} \subseteq \mathcal{C}(I)] \leqslant } & {\left[\mathcal{B}(I): \mathcal{A}(I)^{c c} \otimes \mathcal{A}(I)^{c}\right] } \\
& \cdot\left[\mathcal{A}(I)^{c c} \otimes \mathcal{A}(I)^{c}: \mathcal{A} \subseteq \mathcal{C}(I)\right], \\
{\left[\mathcal{A}(I)^{c c} \otimes \mathcal{A}(I)^{c}: \mathcal{A} \subseteq \mathcal{C}(I)\right]=} & {\left[\mathcal{A}(I)^{c c}: \mathcal{A}(I)\right]\left[\mathcal{A}(I)^{c}: \mathcal{C}(I)\right] . }
\end{aligned}
$$


The inequality (V.8) is saturated, if $\mathcal{A} \subset \mathcal{C}(I) \subset \mathcal{B}(I)$ is irreducible. This is the case: the index $[\mathcal{B}(I): \mathcal{A} \subset \mathcal{C}(I)]$ is finite and hence the relative commutant of $\mathcal{A} \subset \mathcal{C}(I)$ in $\mathcal{B}(I)$ is finite dimensional [Lon90]. This finite-dimensional algebra is modular covariant and hence, according to lemma A.2, all vectors in $\mathcal{A} \subset \mathcal{C}(I)^{c} \Omega$ are invariant with respect to the translation group $U\left(T_{I}().\right)$ (cf proof of proposition IV.6), but this means that they are fixed by all of $U$ (lemma A.1). Uniqueness of the vacuum and its separating property show that the relative commutant is trivial. The other inclusions are irreducible by the same argument.

Inclusions of infinite subfactors $\mathcal{N} \subset \mathcal{M}$ with finite index may be characterised in terms of intertwiners between endomorphisms. If $\rho, \sigma$ are endomorphisms of $\mathcal{M}$, then an intertwiner from $\rho$ to $\sigma$ is a bounded operator $T$ which satisfies for all $m \in \mathcal{M}: T \rho(m)=\sigma(m) T$. This property is indicated by the notation $T: \rho \rightarrow \sigma$. The following may be found eg in [LR95, RST96, Reh94b], where some more useful identities on the intertwiners are stated as well. We take $\mathcal{N} \subset \mathcal{M}$ to be irreducible :

The subfactor $\mathcal{N} \subset \mathcal{M}$ is completely determined by the triple $(\gamma, v, w)$ where $\gamma$ is an endomorphism of $\mathcal{M}, v, w \in \mathcal{M}$ are isometries, $v: i d \rightarrow \gamma, w: \gamma \rightarrow \gamma^{2}$ and the following identities are satisfied:

i) $w^{*} v=[\mathcal{M}: \mathcal{N}]^{-\frac{1}{2}} \mathbb{1}=w^{*} \gamma(v)$,

ii) $w w^{*}=\gamma\left(w^{*}\right) w$,

iii) $w w=\gamma(w) w$.

One may reconstruct $\mathcal{N}$ as the image of $\mu()=.w^{*} \gamma()$.$w . Every m \in \mathcal{M}$ is of the form:

$$
m=[\mathcal{M}: \mathcal{N}] \mu\left(m v^{*}\right) v=[\mathcal{M}: \mathcal{N}] v^{*} \mu(v m)
$$

The dual canonical endomorphism $\rho$ has a finite decomposition into irreducible, inequivalent endomorphisms $\rho_{s}, \rho \cong \bigoplus_{s} N_{s} \rho_{s}$. For every $\rho_{s}$ there is a complete orthonormal set of intertwiners $\mathcal{N} \ni w_{s}^{i}: \rho_{s} \rightarrow \rho, i=1, \ldots, N_{s}$, $\sum_{i s} w_{s}^{i} w_{s}^{i *}=\mathbb{1}, w_{s}^{i *} w_{t}^{j}=\delta_{s t} \delta^{i j} \mathbb{1}$. The second equation defines a scalar product on the intertwiner spaces.

The definition $\psi_{s}^{i}:=w_{s}^{i *} v$ yields an anti-isomorphism between the intertwiner spaces of the $w_{s}^{i}$ and the space of charged intertwiners, spanned by the $\psi_{s}^{i}: i d \rightarrow$ $\rho_{s}$ ([LR95, lemma 4.5], [Reh94b, proposition 2]). By irreducibility of $\mathcal{N} \subset \mathcal{M}$, there is a scalar product on the space of charged intertwiners:

$$
\left\langle\psi_{s_{1}}^{i_{1}}, \psi_{s_{2}}^{i_{2}}\right\rangle \mathbb{1} \equiv \psi_{s_{1}}^{i_{1} *} \psi_{s_{2}}^{i_{2}} \in \mathcal{N}^{\prime} \cap \mathcal{M}=\mathbb{C} \mathbb{1} .
$$

Equation (V.10) leads to a decomposition of $\mathcal{M}$ with respect to the $\rho_{s}$ :

$$
m=\sum_{s, i}[\mathcal{M}: \mathcal{N}] \mu\left(m \psi_{s}^{i *}\right) \psi_{s}^{i} .
$$

This formula is the ground for the following proposition. 
Proposition V.2: Let $\mathcal{A} \subseteq \mathcal{C} \subset \mathcal{B}$ be a COSEt pair of finite index. Assume the corresponding inner-implementing representations $U^{\mathcal{A}}, U^{\mathcal{C}}$ to have the netendomorphism property. Then the local inclusions $\mathcal{A} \subseteq \mathcal{C}(I) \subset \mathcal{B}(I)$ are irreducible, $\mathcal{A}_{\max }(I)$ and $\mathcal{C}_{\max }(I)$ are their mutual relative commutants and we have: $U \circ \mathrm{p}=U^{\mathcal{A}} U^{\mathcal{C}}$.

Proof: The irreducibility of $\mathcal{A} \subset \mathcal{C}(I) \subset \mathcal{B}(I)$ was shown in lemma V.1.

Exploiting covariance, we restrict our attention to the case $I=\mathrm{S}_{+}^{1}$. We denote elements in $\mathcal{A} \subseteq \mathcal{C}\left(\mathrm{S}_{+}^{1}\right)$ by $[a c]$. Each $b \in \mathcal{B}\left(\mathrm{S}_{+}^{1}\right)$ has the form $b=\sum_{s, i}[a c]_{s, i} \psi_{s}^{i}$. By assumption, $A d_{U \mathcal{A}^{\prime} \cap \mathcal{C}^{\prime}}(\tilde{D}(\tau))$ (see equation (II.21) for the definition of $U^{\mathcal{A}^{\prime} \cap \mathcal{C}^{\prime}}$ ) defines an automorphism of $\mathcal{B}\left(\mathrm{S}_{+}^{1}\right)$, which acts trivial on $\mathcal{A} @ \mathcal{C}\left(\mathrm{S}_{+}^{1}\right)$ and leaves globally invariant the finite-dimensional spaces of charged intertwiners $\psi_{s}^{i}$. According to the lemmas A.2 and A.1 the vectors $\psi_{s}^{i} \Omega$ are invariant with respect to $U^{\mathcal{A}^{\prime} \cap \mathcal{C}^{\prime}}$. The ReEH-Schlieder theorem on the chiral theory $\mathcal{B}$ and the decomposition $b=\sum_{s, i}[a c]_{s, i} \psi_{s}^{i}$ for each $b \in \mathcal{B}\left(\mathrm{S}_{+}^{1}\right)$ yields $U^{\mathcal{A}^{\prime} \cap \mathcal{C}^{\prime}}=\mathbb{1} \Leftrightarrow U \circ \mathrm{p}=U^{\mathcal{A}} U^{\mathcal{C}}$.

The same line of argument works for the inclusion $\mathcal{A}_{\max }\left(\mathrm{S}_{+}^{1}\right) \subset \mathcal{A}\left(\mathrm{S}_{+}^{1}\right)^{c c}$, where it proves every element in $\mathcal{A}\left(\mathrm{S}_{+}^{1}\right)^{c c}$ to be invariant with respect to $A d_{U^{c}}$ and hence to be contained in $\mathcal{A}_{\max }\left(\mathrm{S}_{+}^{1}\right)$ (invoking proposition IV.6). The proof is complete, if we apply the same routine to $\mathcal{C}_{\max }\left(\mathrm{S}_{+}^{1}\right) \subset \mathcal{A}\left(\mathrm{S}_{+}^{1}\right)^{c}$.

Proposition V.2 establishes a complete analogy between the Coset construction of stress-energy tensors for current subalgebras of Goddard, Kent, Olive [GKO86] and the Borchers-Sugawara representations of a CoSET pair of finite index: The identity $U \circ \mathrm{p}=U^{\mathcal{A}} U^{\mathcal{C}}$ shows that $U$ possesses a factorisation with respect to the COSET pair $\mathcal{A} \subseteq \mathcal{C} \subset \mathcal{B}$ which is generated by commuting local observables (cf discussion in section II,2.2). Furthermore, it shows that the net-endomorphism property is sufficient to solve the isotony problem for COSET pairs of finite index and to prove that the maximal CosET pair is normal.

The proof of proposition V.2 is similar to the ones in the well-known situation of current subalgebras for which the vacuum representation of the larger one decomposes into finitely many irreducible representations when restricted to the subalgebra (see eg [KW88, corollary 3.2.1]): loosely speaking, the assumption of finite index trivialises the problem, since there are no non-trivial finite-dimensional, unitary representations of $\operatorname{PSL}(2, \mathbb{R})^{\sim}$.

\section{Coset pairs as nets of subfactors}

In this section we study some aspects of the inclusion of CosET pairs as left and right chiral observables in the 1+1-dimensional quasi-theory $\mathcal{B}^{1+1}$ constructed from the subnet $\mathcal{A} \subset \mathcal{B}$ by means of chiral holography (section IV, 2 ). Our arguments naturally apply to inclusions of left and right chiral observables in a 1+1-dimensional conformal theory as well, and we try to make this analogy 
manifest in the following. Hence, we make contact with structures discussed by REHREN [Reh00], who has analysed the inclusion of chiral observables in a (genuine) 1+1-dimensional conformal theory.

In particular, we investigate the character of the inclusion $\mathcal{A} \subset \mathcal{C} \subset \mathcal{B}^{1+1}$ as a localisable representation of $\mathcal{A} \otimes \mathcal{C}$, which is considered as a $1+1$-dimensional theory. The assumption of finite index for the COSET pair forms the ground for most of the following. If the Coset pair is spatial (see below) in addition, then we can improve easily our knowledge on the sub-geometrical action of $U^{\mathcal{A}}$ on $\mathcal{B}$ following from the net-endomorphism property (proposition IV.6) and show that the spectrum of $U^{\mathcal{A}}(\tilde{R}(2 \pi))$ has to be contained in $\{ \pm 1\}$, if $U^{\mathcal{A}}$ has sharp geometrical action.

We proceed along the lines of [LR95, RST96, Reh94b, GL96] and, in particular, [Lon01]. Some of the discussions below are given merely for the convenience of the reader, as most of the arguments in [Lon01] are adapted easily from the chiral setting to the 1+1-dimensional situation, or essentially stem from [LR95] where the formulation does not refer to a specific spacetime at all.

\subsection{The localised representation of a COSET pair}

In section 1 we considered inclusions of local algebras in the chiral case; now, we want to take the net-structure of inclusions into account and we do that for the inclusion of the Coset pair $\mathcal{A}\left(\mathcal{C}\right.$ in the $1+1$-dimensional quasi-theory $\mathcal{B}^{1+1}$. In the terminology of LONGO AND REHREN, who developed the general, abstract framework of nets of subfactors [LR95], our subnets are particular examples of (irreducible) quantum field theoretical nets of subfactors. We have to extend the arguments of [LR95] from directed nets of subfactors to the situation in the 1+1-dimensional conformal covering space.

The assumption of conformal covariance on chiral subnets (definition II.2) implies that we have a conditional expectation for each local inclusion which is normal, faithful and leaves invariant the vacuum. These conditional expectations reflect the net-structure:

The reconstruction of each conditional expectation $\mu_{I}: \mathcal{B}(I) \rightarrow \mathcal{A}(I)$ through the cyclic projection $e_{\mathcal{A}}$ (see vi) on page 84) shows that the conditional expectations are consistent with the net of inclusions: $\mu_{I} \uparrow \mathcal{B}(J)=\mu_{J}, J \subset I$. Namely, for $b \in \mathcal{B}(J) \subset \mathcal{B}(I)$ the equation $e_{\mathcal{A}} b e_{\mathcal{A}}=a e_{\mathcal{A}}$ has only one solution $a \in \mathcal{A}(J) \subset \mathcal{A}(I)$, namely $a=\mu_{I}(b)=\mu_{J}(b)$. The same line of argument proves the conditional expectations to be covariant:

$$
\alpha_{g}\left(\mu_{I}(b)\right) e_{\mathcal{A}}=\alpha_{g}\left(e_{\mathcal{A}} b e_{\mathcal{A}}\right)=e_{\mathcal{A}} \alpha_{g}(b) e_{\mathcal{A}}=\mu_{g I}\left(\alpha_{g}(b)\right) e_{\mathcal{A}} .
$$

These structures carry over to the 1+1-dimensional situation, which we study now.

Let $\mathcal{B}^{1+1}$ be a $1+1$-dimensional conformal (quasi-) theory (cf section IV 2 ) and denote its restriction to MinkOWski space by $\mathcal{B}_{0}^{1+1}$, ie we look at the (directed) 
net of local algebras $\mathcal{B}^{1+1}(\mathcal{O}), \mathcal{O}$ a bounded double cone in $\mathbb{M}$, denoted $\mathcal{O} \Subset \mathbb{M}$. Bounded double cones are Cartesian products $I \times J$ of bounded intervals on the chiral light-rays. The localisation regions of $\mathcal{B}^{1+1}$ are double cones $\mathcal{O}$ which are properly contained a single copy of $\mathbb{M}$; such double cones will be indicated as $\mathcal{O} \Subset \widetilde{\mathbb{M}}$.

Suppose, we have a localised representation $\rho$ of $\mathcal{B}^{1+1}$, that is: for each double cone $\mathcal{O} \Subset \widetilde{\mathbb{M}}$ the algebra $\rho_{\mathcal{O}}\left(\mathcal{B}^{1+1}(\mathcal{O})\right)$ forms a normal representation of the respective local algebra $\mathcal{B}^{1+1}(\mathcal{O})$ on the vacuum HILBERT space $\mathscr{H}$ of $\mathcal{B}^{1+1}$, and the $\rho_{\mathcal{O}}$ fulfill:

○ consistency: $\mathcal{O}_{1} \subset \mathcal{O}_{2} \Rightarrow \rho_{\mathcal{O}_{2}}\left\lceil\mathcal{B}^{1+1}\left(\mathcal{O}_{1}\right)=\rho_{\mathcal{O}_{1}}\right.$,

○ locality: $\mathcal{O}_{1} \subset \mathcal{O}_{2}^{\prime} \Rightarrow \rho_{\mathcal{O}_{1}}\left(\mathcal{B}^{1+1}\left(\mathcal{O}_{1}\right)\right) \subset \rho_{\mathcal{O}_{2}}\left(\mathcal{B}^{1+1}\left(\mathcal{O}_{2}\right)\right)^{\prime}$,

○ localisation: for some $\mathcal{O}_{0}^{\prime} \Subset \widetilde{\mathbb{M}}$ we have $\rho_{\mathcal{O}}=i d_{\mathcal{O}}$ for all $\mathcal{O} \subset \mathcal{O}_{0}^{\prime}$.

By HAAG duality of $\mathcal{B}^{1+1}$ [BGL93], $\rho$ satisfies the following properties:

i) If $\mathcal{O} \Subset \widetilde{\mathbb{M}}, \mathcal{O}_{0} \subset \mathcal{O}$, then $\rho_{\mathcal{O}}\left(\mathcal{B}^{1+1}(\mathcal{O})\right) \subset \mathcal{B}^{1+1}(\mathcal{O})$, and for all $\mathcal{O}_{1} \Subset \widetilde{\mathbb{M}}$ containing $\mathcal{O}$ we have: $\rho_{\mathcal{O}_{1}}\left\lceil\mathcal{B}^{1+1}(\mathcal{O})=\rho_{\mathcal{O}}\right.$.

ii) If $\mathcal{O} \subset \mathcal{O}_{0}^{\prime}$, we have $\rho_{\mathcal{O}}=i d_{\mathcal{O}}$.

iii) For $\mathcal{O}_{1}, \mathcal{O}_{2} \Subset \widetilde{\mathbb{M}}, \mathcal{O}_{0} \cup \mathcal{O}_{1} \subset \mathcal{O}_{2}$, there is a unitary $u \in \mathcal{B}^{1+1}\left(\mathcal{O}_{2}\right)$ such that $\mathcal{O} \mapsto \tilde{\rho}_{\mathcal{O}}():.=u \rho_{\mathcal{O}}(.) u^{*}$ is a representation localised in $\mathcal{O}_{1}$.

We call any map $\rho$ which associates with every $\mathcal{O} \Subset \mathbb{M}$ a normal representation $\rho_{\mathcal{O}}$ of $\mathcal{B}^{1+1}(\mathcal{O})$ and which satisfies i), ii), iii) with $\widetilde{\mathbb{M}}$ replaced by $\mathbb{M}$ a $D H R$ endomorphism (of $\mathcal{B}_{0}^{1+1}$ ), in direct correspondence with the works of DopLICHER, HaAG, RoberTs [DHR69a, DHR69b, DHR71, DHR74].

It will prove possible to construct a DHR endomorphism for $\mathcal{A} \otimes \mathcal{C}_{0}$ from the inclusion $\mathcal{A} \subseteq \mathcal{C} \subset \mathcal{B}^{1+1}$ and we need to extend this to a representation of $\mathcal{A} \otimes \mathcal{C}$. In the following lemma $\mathbb{M}_{0, \pm}$ stands for the open interior of the closure of the union of $\mathbb{M}$ and the two neighbouring sheets of the covering as indicated in figure V.1; the restriction of $\mathcal{B}^{1+1}$ to $\mathbb{M}_{0, \pm}$ is denoted by $\mathcal{B}_{0, \pm}^{1+1}$. We state the lemma in some more generality than actually needed in the following:

Lemma V.3: Let $\rho$ be a DHR endomorphism of $\mathcal{B}_{0}^{1+1}$ localised in some $\mathcal{O}_{0} \Subset \mathbb{M}$. There is a unique representation of $\mathcal{B}_{0, \pm}^{1+1}$ extending $\rho$ and localised in $\mathcal{O}_{0}$.

Proof: The argument is straightforward and virtually identical to the chiral case [Lon01, proposition 11]. For geometrical details see figure V.1, where points on the left and right boundary are to be identified if they have the same height.

For each $\mathcal{O} \Subset \mathbb{M}_{0, \pm}$ there is a $\mathbb{M} \ni \mathcal{O}_{1} \subset \mathcal{O}^{\prime}$ and a $\mathcal{O}_{2} \Subset \mathbb{M}$ containing both $\mathcal{O}_{0}$ and $\mathcal{O}_{1}$. Transporting $\rho$ from $\mathcal{O}_{0}$ to $\mathcal{O}_{1}$ we set with respect to a corresponding 


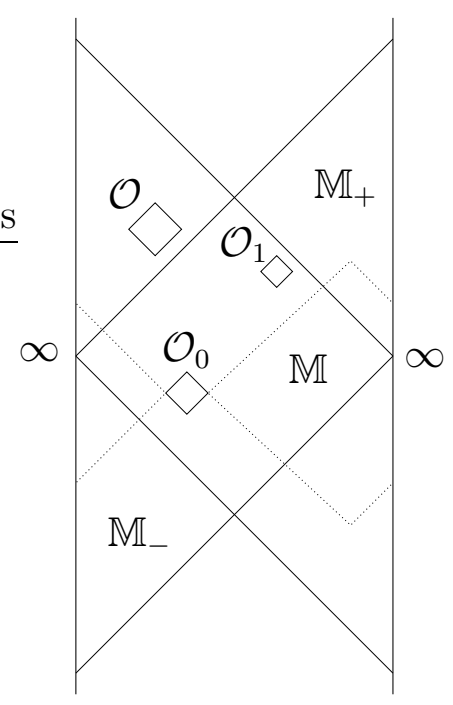

Figure V.1

$u \in \mathcal{B}^{1+1}\left(\mathcal{O}_{2}\right): \tilde{\rho}:=A d_{u} \circ \rho$. The extension of $\rho$ to $\mathcal{B}^{1+1}(\mathcal{O}), \mathcal{O} \Subset \mathbb{M}_{0, \pm}$, is defined by $\rho_{\mathcal{O}}:=A d_{u^{*}}$.

Obviously, this definition extends $\rho$. It is well defined as a different choice of $u, \mathcal{O}_{1}, \mathcal{O}_{2}$ for $\rho$ 's transport results in adjoining a local unitary associated with the causal complement of $\mathcal{O}$. The extension is localised in $\mathcal{O}_{0}$ since for $\mathcal{O} \subset \mathcal{O}_{0}^{\prime}$ we may choose $u=\mathbb{1}, \mathcal{O}_{0}=\mathcal{O}_{1}=\mathcal{O}_{2}$. The consistency of the extension for $\mathcal{O}_{a} \subset \mathcal{O}_{b}$ is seen easily by a common choice $u, \mathcal{O}_{1}, \mathcal{O}_{2}$.

Locality of $\rho$ follows in this way: For any pair $\mathcal{O}_{a}, \mathcal{O}_{b} \Subset \mathbb{M}_{0, \pm}, \mathcal{O}_{b} \subset \mathcal{O}_{a}^{\prime}$ there is a covering $\left\{\mathcal{O}_{b}^{i}\right\}$ of $\mathcal{O}_{b}$ by double cones $\mathcal{O}_{b}^{i}$ such that $\overline{\mathcal{O}_{b}^{i}} \subset \mathcal{O}_{b}$ and there is a double cone $\mathcal{O}_{1}^{i} \Subset \mathbb{M}$ spacelike to both $\mathcal{O}_{a}$ and $\mathcal{O}_{b}^{i}$. Transporting $\rho$ from $\mathcal{O}_{0}$ into $\mathcal{O}_{1}^{i}$ as above, we have:

$$
\tilde{\rho}_{\mathcal{O}_{b}^{i}}^{i}\left(\mathcal{B}^{1+1}\left(\mathcal{O}_{b}^{i}\right)\right)=\mathcal{B}^{1+1}\left(\mathcal{O}_{b}^{i}\right) \subset \mathcal{B}^{1+1}\left(\mathcal{O}_{a}\right)^{\prime}=\tilde{\rho}_{\mathcal{O}_{a}}^{i}\left(\mathcal{B}^{1+1}\left(\mathcal{O}_{a}\right)\right)^{\prime}
$$

Now one has to use the unitary equivalence of $\tilde{\rho}^{i}$ and $\rho$ and weak additivity of $\mathcal{B}^{1+1}$, which yields: $\rho_{\mathcal{O}_{b}}\left(\mathcal{B}^{1+1}\left(\mathcal{O}_{b}\right)\right) \subset \rho_{\mathcal{O}_{a}}\left(\mathcal{B}^{1+1}\left(\mathcal{O}_{a}\right)\right)^{\prime}$.

Finally, we verify the uniqueness of the extension. For arbitrary $\mathcal{O} \subset \mathbb{M}_{0, \pm}$, we transport $\rho$ into $\mathcal{O}_{1} \subset \mathcal{O}^{\prime}$ and define $\tilde{\rho}$ as above. If $\rho^{(1)}, \rho^{(2)}$ are two extensions of $\rho$, we look at $\tilde{\rho}^{(1),(2)}:=A d_{u} \circ \rho^{(1),(2)}$. Since $\tilde{\rho}^{(1),(2)}$ are localised in $\mathcal{O}_{1} \subset \mathcal{O}^{\prime}$, they agree trivially on $\mathcal{B}^{1+1}(\mathcal{O})$, namely: $\tilde{\rho}_{\mathcal{O}}^{(1)}=i d \uparrow \mathcal{B}^{1+1}(\mathcal{O})=\tilde{\rho}_{\mathcal{O}}^{(2)}$. The remainder follows from the definition of $\tilde{\rho}^{(1),(2)}$.

Remarks: Restricting to time-zero double cones yields the chiral case [Lon01, proposition 11]; here the complete conformal covering space, $\mathrm{S}^{1}$, is reached by the argument.

It is seen easily, that the extended $\rho$ may be transported into any $\mathcal{O}_{1}$ for which $\mathcal{O}_{1}, \mathcal{O}_{1}^{\prime} \Subset \mathbb{M}_{0, \pm}$ by the usual procedure relying on local normality. This leads us 
to recognise that $\rho$ may not be extended beyond $\mathbb{M}_{0, \pm}$ by the procedure in the proof of lemma V.3. Fortunately, this is not needed for our purposes either.

In the following, we extend the dual canonical endomorphism of a local inclusion $\mathcal{A} \subset \mathcal{C}\left(\mathcal{O}_{0}\right) \subset \mathcal{B}^{1+1}\left(\mathcal{O}_{0}\right)$ to a DHR endomorphism of $\mathcal{A} \otimes \mathcal{C}_{0}$. Lemma V.3 and the periodicity of $\mathcal{A} \otimes \mathcal{C}$ over $\widetilde{\mathbb{M}}$ then tell us that this DHR endomorphism extends to a localised representation.

Lemma V.4: Choose a canonical endomorphism $\gamma_{\mathcal{O}_{0}}: \mathcal{B}^{1+1}\left(\mathcal{O}_{0}\right) \rightarrow \mathcal{A} \subset \mathcal{C}\left(\mathcal{O}_{0}\right)$. Then for any $\mathcal{O} \supset \mathcal{O}_{0}$ there is a canonical endomorphism $\gamma_{\mathcal{O}}$ satisfying: $\gamma_{\mathcal{O}} \uparrow$ $\mathcal{B}^{1+1}\left(\mathcal{O}_{0}\right)=\gamma_{\mathcal{O}_{0}}$.

If the COSET pair is of finite index, there are common isometric intertwiners

$$
\mathcal{B}^{1+1}\left(\mathcal{O}_{0}\right) \ni v: i d \rightarrow \gamma_{\mathcal{O}}, \mathcal{A} \subset \mathcal{C}\left(\mathcal{O}_{0}\right) \ni w: i d \rightarrow \rho_{\mathcal{O}}
$$

Proof: (Along the lines of [Lon01, lemma 15]) We set $\mathcal{M}:=\mathcal{B}^{1+1}(\mathcal{O}), \mathcal{M}^{0}:=$ $\mathcal{B}^{1+1}\left(\mathcal{O}_{0}\right), \mathcal{N}:=\mathcal{A} \subset \mathcal{C}(\mathcal{O}), \mathcal{N}^{0}:=\mathcal{A} \subset \mathcal{C}\left(\mathcal{O}_{0}\right)$. Moreover, we define $\mathcal{M}_{1}:=\mathcal{M} \vee$ $\left\{e_{\mathcal{N}}\right\}^{\prime \prime}, \mathcal{M}_{1}^{0}:=\mathcal{M}^{0} \vee\left\{e_{\mathcal{N}}\right\}^{\prime \prime}$. Let $\mu: \mathcal{M} \rightarrow \mathcal{N}, \mu^{0}: \mathcal{M}^{0} \rightarrow \mathcal{N}^{0}$ be the normal, faithful conditional expectations which leave the vacuum invariant.

There is an isometry $v_{1} \in \mathcal{M}_{1}^{0}$ satisfying $v_{1} v_{1}^{*}=e_{\mathcal{N}}=e_{\mathcal{N}^{0}}$ which reconstructs $\gamma_{\mathcal{O}_{0}}$ by the unique solutions in $\mathcal{A} \subset \mathcal{C}\left(\mathcal{O}_{0}\right)$ of the following equation on elements $b \in \mathcal{B}^{1+1}\left(\mathcal{O}_{0}\right)$ [LR95, proposition 2.9, theorem 3.2]:

$$
\gamma_{\mathcal{O}_{0}}(b) e_{\mathcal{N}} \equiv v_{1} b v_{1}^{*}
$$

The same equation for elements $b \in \mathcal{B}^{1+1}(\mathcal{O})$ defines a canonical endomorphism $\gamma_{\mathcal{O}}$ of $\mathcal{B}^{1+1}(\mathcal{O})$. Consistency of $\gamma_{\mathcal{O}_{0}}$ and $\gamma_{\mathcal{O}}$ is obvious.

In case the index is finite, there exist normal, faithful conditional expectations $\mu_{1}: \mathcal{M}_{1} \rightarrow \mathcal{M}, \mu_{1}^{0}: \mathcal{M}_{1}^{0} \rightarrow \mathcal{M}^{0}$ with the same (minimal) index. These are consistent: in $\mathcal{M}_{1}^{0}$ elements of the form $\sum_{i} x_{i} e_{\mathcal{N}} y_{i}, x_{i}, y_{i} \in \mathcal{M}^{0}$ are dense [Jon83, PP86] and we have $\mu_{1}\left(e_{\mathcal{N}}\right)=[\mathcal{M}: \mathcal{N}]^{-1}=\mu_{1}^{0}\left(e_{\mathcal{N}}\right)$. Consistency follows as a consequence of normality from the following identity:

$$
\mu_{1}\left(\sum_{i} x_{i} e_{\mathcal{N}} y_{i}\right)=\sum_{i} x_{i} \mu_{1}\left(e_{\mathcal{N}}\right) y_{i}=\mu_{1}^{0}\left(\sum_{i} x_{i} e_{\mathcal{N}} y_{i}\right)
$$

The operators $v, w$ are obtained as follows (see [LR95, (2.16)]):

$$
\begin{aligned}
{[\mathcal{M}: \mathcal{N}]^{-\frac{1}{2}} v } & :=\mu_{1}\left(v_{1}\right)=\mu_{1}^{0}\left(v_{1}\right) \\
{[\mathcal{M}: \mathcal{N}]^{-\frac{1}{2}} w } & :=\mu(v)=\mu^{0}(v)
\end{aligned}
$$

They fulfill the relations i), ii), iii) on page 86 . 
Lemma V.5: Let $v_{1} \in \mathcal{B}^{1+1}\left(\mathcal{O}_{0}\right) \vee\left\{e_{\mathcal{A} \odot \mathcal{C}}\right\}^{\prime \prime}$ be an isometry satisfying $v_{1} v_{1}^{*}=$ $e_{\mathcal{A} \odot \mathcal{C}}$. The dual canonical endomorphism $\rho_{\mathcal{O}_{0}}$ defined by

$$
\rho_{\mathcal{O}_{0}}([a c]) e_{\mathcal{A} @ \mathcal{C}}=v_{1}[a c] v_{1}^{*}, \quad[a c] \in \mathcal{A}\left(\mathcal{C}\left(\mathcal{O}_{0}\right)\right.
$$

has an extension defining a localised representation of $\mathcal{A} \otimes \mathcal{C}$ localised in $\mathcal{O}_{0}$, which shall be called $\rho$.

Proof: (Along the lines of [Lon01, proposition 16].) We choose a sheet of the covering as Minkowski space, $\mathbb{M}$, containing $\mathcal{O}_{0}$ as a bounded double cone. The extension of $\rho_{\mathcal{O}_{0}}$ to all of $\mathcal{A} \subset \mathcal{C}_{0}$ is constructed as in the proof of lemma $\mathrm{V} .4$ by extending the canonical endomorphism $\gamma_{\mathcal{O}_{0}}$. The isomorphisms between the local algebras of $\mathcal{A} @ \mathcal{C}_{0}$ and $\mathcal{A} \otimes \mathcal{C}_{0}$ define the action of the extension of $\rho_{\mathcal{O}_{0}}$ on $\mathcal{A} \otimes \mathcal{C}_{0}$ (see $\mathrm{v}$ ), page 84) and hence of $\rho$ itself.

We have to check the conditions i), ii), iii) (page 89) for $\rho$. i) and ii) are contained in [LR95, theorem 3.2]; condition iii) follows from the uniqueness of the canonical endomorphism up to unitary equivalence with respect to a local unitary in $\mathcal{A}\left(\mathcal{C}_{0}\right.$ [Lon87].

Finally, we apply lemma $\mathrm{V} .3$ and recognise the periodicity of $\mathcal{A} \otimes \mathcal{C}$ on conformal covering space which means that $\mathcal{A} \otimes \mathcal{C}$ effectively lives on $\mathrm{S}^{1} \times \mathrm{S}^{1}$ and hence extension to $\mathbb{M}_{0, \pm}$ is sufficient.

We have $\rho_{\mathcal{O}}(\mathcal{A} \otimes \mathcal{C}(\mathcal{O})) \subset \mathcal{A} \otimes \mathcal{C}(\mathcal{O})$ for each $\mathcal{O} \supset \mathcal{O}_{0}$, because $\rho$ is localised and $\mathcal{A} \otimes \mathcal{C}$ satisfies HaAg duality. Moreover, $[a c] \mapsto[a c] e_{\mathcal{A} \odot \mathcal{C}},[a c] \in \mathcal{A} \subset \mathcal{C}(\mathcal{O})$, $\mathcal{O} \Subset \widetilde{\mathbb{M}}$, defines an isomorphism of v.NEUMANN algebras which can be used to define:

$$
\rho_{\mathcal{O}}([a c]) e_{\mathcal{A} \odot \mathcal{C}} \equiv \rho_{\mathcal{O}}\left([a c] e_{\mathcal{A} \odot \mathcal{C}}\right), \mathcal{O} \supset \mathcal{O}_{0}
$$

It is clear that this definition agrees with the dual canonical endomorphisms for $\mathcal{O} \Subset \mathbb{M}$ which were used to construct $\rho$ (see proof of lemma V.5).

Proposition V.6: The representation $\rho$ of lemma $V .5$ is unitarily equivalent to the representation $\mathcal{A} \subset \mathcal{C} \subset \mathcal{B}^{1+1}$. In particular, it is covariant with the same spectrum condition on the translations. It holds true for $v_{1}$ as above and $\mathcal{O} \Subset \widetilde{\mathbb{M}}$ :

$$
\rho_{\mathcal{O}}\left([a c] e_{\mathcal{A} \odot \mathcal{C}}\right)=v_{1}[a c] v_{1}^{*}, \quad[a c] \in \mathcal{A} \subset \mathcal{C}(\mathcal{O})
$$

Proof: (Along the lines of [Lon01, proposition 17].) For $\mathcal{O}_{0} \subset \mathcal{O} \Subset \mathbb{M}$ and for $\mathcal{O} \subset \mathcal{O}_{0}^{\prime}$ there is nothing to show. For the general case we follow the same strategy as in the proof of lemma V.3. It suffices to look at proper double cones $\mathcal{O}$ in $\mathbb{M}_{0, \pm}$.

We choose $\mathbb{M} \ni \mathcal{O}_{1} \subset \mathcal{O}^{\prime}$ and $\mathcal{O}_{2} \Subset \mathbb{M}$ containing both $\mathcal{O}_{1}$ and $\mathcal{O}_{0}$. There is a canonical endomorphism $\gamma_{\mathcal{O}_{2}}$ which extends $\gamma_{\mathcal{O}_{0}}$ and yields through its restriction to $\mathcal{A} \subset \mathcal{C}\left(\mathcal{O}_{2}\right)$, the dual canonical endomorphism $\rho_{\mathcal{O}_{2}}$, the same localised representation $\rho$ by the process described in the proof of lemma V.5. 
Now choose a canonical endomorphism $\tilde{\gamma}_{\mathcal{O}_{1}}$. According to lemma V.4 there is a canonical endomorphism $\tilde{\gamma}_{\mathcal{O}_{2}}$ which extends $\tilde{\gamma}_{\mathcal{O}_{1}}$, and there is a unitary $u \in \mathcal{A}\left(\mathcal{C}\left(\mathcal{O}_{2}\right)\right.$ such that $\tilde{\gamma}_{\mathcal{O}_{2}}=A d_{u} \circ \gamma_{\mathcal{O}_{2}}$ [Lon87]. The defining isometry $\tilde{v}_{1} \in \mathcal{B}^{1+1}\left(\mathcal{O}_{1}\right) \vee\left\{e_{\mathcal{A} \odot \mathcal{C}}\right\}^{\prime \prime}$ of $\tilde{\gamma}_{\mathcal{O}_{1}}$ satisfies: $\tilde{v}_{1}=u v_{1}$.

The dual canonical endomorphism $\tilde{\rho}_{\mathcal{O}_{1}}$ extends to a localised representation $\tilde{\rho}$ for which we have: $\tilde{\rho}=A d_{u} \circ \rho$. For $[a c] \in \mathcal{A} \subset \mathcal{C}(\mathcal{O})$ this leads to:

$$
u \rho_{\mathcal{O}}\left([a c] e_{\mathcal{A} \odot \mathcal{C}}\right) u^{*}=\tilde{\rho}\left([a c] e_{\mathcal{A} \odot \mathcal{C}}\right)=[a c] e_{\mathcal{A} \odot \mathcal{C}}=\tilde{v}_{1}[a c] \tilde{v}_{1}^{*}=u v_{1}[a c] v_{1}^{*} u^{*} .
$$

The following lemma determines to a large extent the transformation behaviour of observables in $\mathcal{B}^{1+1}$ with respect to chiral transformations, provided the Coset pair has finite index:

Lemma V.7: Assume $\left[\mathcal{B}^{1+1}: \mathcal{A} \subseteq \mathcal{C}\right]<\infty$, and take $v \in \mathcal{B}^{1+1}\left(\mathcal{O}_{0}\right), w \in \mathcal{A} \subseteq \mathcal{C}\left(\mathcal{O}_{0}\right)$ as in equations (V.14), (V.15). If $\mathcal{O} \Subset \widetilde{\mathbb{M}}$ contains $\mathcal{O}_{0}$, we have for all $[a c] \in$ $\mathcal{A}(\mathcal{C}(\mathcal{O}):$

$$
\rho_{\mathcal{O}}([a c]) v_{1}=v_{1}[a c], \rho_{\mathcal{O}}([a c]) v=v[a c], \rho_{\mathcal{O}}([a c]) w=w[a c] .
$$

Proof: From equations (V.17) and (V.18) follows immediately the first statement in (V.19). For the remainder apply the conditional expectations $\mu_{1}: \mathcal{B}^{1+1}\left(\mathcal{O}_{1}\right) \vee$ $\left\{e_{\mathcal{A} \odot \mathcal{C}}\right\}^{\prime \prime} \rightarrow \mathcal{B}^{1+1}\left(\mathcal{O}_{1}\right)$ and $\mu \mu_{1}: \mathcal{B}^{1+1}\left(\mathcal{O}_{1}\right) \vee\left\{e_{\mathcal{A} \odot \mathcal{C}}\right\}^{\prime \prime} \rightarrow \mathcal{A} \subseteq \mathcal{C}\left(\mathcal{O}_{1}\right)$ (see proof of lemma $\mathrm{V} .4)$.

The following proposition is practically identical to [Lon01, corollary 18]:

Proposition V.8: Assume $\left[\mathcal{B}^{1+1}: \mathcal{A} \subseteq \mathcal{C}\right]<\infty$. $\rho$ shall denote a representation of $\mathcal{A} \otimes \mathcal{C}$ on $e_{\mathcal{A} \odot \mathcal{C}} \mathscr{H}$ which is unitarily equivalent to $\mathcal{A} \subseteq \mathcal{C} \subset \mathcal{B}^{1+1}$ and localised is some double cone $\mathcal{O}_{0}$. Then $\rho$ has finite decomposition into irreducibles:

$$
\rho \cong \bigoplus_{i=0}^{n} N_{i} \rho^{i}
$$

The $\rho^{i}$ are covariant representations of $\mathcal{A} \otimes \mathcal{C}$ with the same spectrum condition on the translations and are localised in $\mathcal{O}_{0}$ alike. For $\mathcal{O} \supset \mathcal{O}_{0}$ the dual canonical endomorphism has the same decomposition:

$$
\rho_{\mathcal{O}} \cong \bigoplus_{i=0}^{n} N_{i} \rho_{\mathcal{O}}^{i}
$$

Proof: Since the spacetime symmetry group of our problem is $\left(P S L(2, \mathbb{R})^{\sim} \times\right.$ $\left.\operatorname{PSL}(2, \mathbb{R})^{\sim}\right) / \mathbb{Z}$ one may adapt the arguments of [GL96] using the propositions and lemmas above. In particular, the identity of global and local intertwiner 
spaces may be proved in the same manner, replacing the choice of one point at infinity by two such points, one on each chiral light-ray. We refrain from repeating the discussions in [GL96].

Remark: Because $\mathcal{A} \subset \mathcal{C} \subset \mathcal{B}^{1+1}$ is irreducible for finite index, the vacuum sector of $\mathcal{A} \otimes \mathcal{C}$ appears exactly once in $\rho$; these two facts are equivalent (see [LR95]).

Before closing this section we only mention very briefly a natural question: For which pairs of chiral nets exist embeddings as COSET pairs in another chiral conformal theory and which are the solutions to this problem? This problem will prove difficult to tackle in general, but if one restricts attention to CosET pairs of finite index there is a detailed formulation.

An irreducible, infinite subfactor $\mathcal{N} \subset \mathcal{M}$ with $[\mathcal{M}: \mathcal{N}]<\infty$ is completely determined by the following data, the $\operatorname{DHR}$ triple $\left(\rho, w, w_{1}\right)$, where $\rho$ is an endomorphism of $\mathcal{N}$ with $d_{\rho}=[\mathcal{M}: \mathcal{N}]$ and $\mathcal{N} \ni w: i d \rightarrow \rho, \mathcal{N} \ni w_{1}: \rho \rightarrow \rho^{2}$ are isometric intertwiners satisfying [Lon94]:

i) $w^{*} w_{1}=[\mathcal{M}: \mathcal{N}]^{-\frac{1}{2}} \mathbb{1}=\rho\left(w^{*}\right) w_{1}$,

ii) $w_{1} w_{1}^{*}=\rho\left(w_{1}^{*}\right) w_{1}$,

iii) $w_{1} w_{1}=\rho\left(w_{1}\right) w_{1}$.

This characterisation is equivalent to the one discussed on page 86, where one has to take $\rho=\gamma \uparrow \mathcal{N}, w_{1}=\gamma(v)$. Concerning chiral subnets and CoseT pairs this has the advantage of determining $\mathcal{B}$ entirely in terms of DHR data of $\mathcal{A}$ or, respectively, of $\mathcal{A} \otimes \mathcal{C}$.

If we now combine some DHR endomorphisms of $\mathcal{A} \otimes \mathcal{C}$ of finite statistics to a reducible endomorphism $\rho$ it is known what is needed in addition to ensure that the extension of $\mathcal{A} \otimes \mathcal{C}$ by means of i), ii), iii) indeed defines a local theory [LR95, theorem 4.9] (some more details in [RST96]). We need to have the following identity for the statistics operator of $\rho, \varepsilon_{\rho}^{<}$, and $w_{1}$ :

$$
\varepsilon_{\rho}^{<} w_{1}=w_{1}
$$

From i), ii), iii) alone only follows relative locality of the extension $\mathcal{B}$. Determining the possible solutions $\mathcal{B}$ is a local extension problem for $\mathcal{A} \otimes \mathcal{C}$. It is outside the purposes of this work to give a more elaborate discussion on this issue. We only mention as introductory references [LR95, RST96, Reh01] and for some examples for solutions of such problems [KL02].

\subsection{On spatial Coset pairs}

It is the purpose of the following discussion to determine the spectrum of the operator $U^{\mathcal{A}}(\tilde{R}(2 \pi))$ in presence of sharp geometrical action under conditions which 
are satisfied in a large set of examples. The spectrum of $U^{\mathcal{A}}(\tilde{R}(2 \pi))$ is known in many examples, and our result excludes the possibility of sharp geometrical action in all these cases (except, of course, the ones that one can make up trivially). On the way we indicate how to improve our knowledge about the geometrical impact of $U^{\mathcal{A}}$ (net-endomorphism property) for cofinite subnets $\mathcal{A} \subset \mathcal{B}$.

The key point is that the transformation behaviour of charged intertwiners is completely given in terms of the COSET pair. This is true in a more general setting than the one we give below, but the derivation of the general statement is a mere repetition of the analysis in the chiral case (see [Lon01, GL96]) and appears to be of little interest. So, we give a discussion aiming directly for our goals, which requires some additional structure. The starting point is

Definition V.9: $\mathcal{A} \subset \mathcal{C} \subset \mathcal{B}$ is called a spatial Coset pair, if the localised representation $\rho$ of $\mathcal{A} \otimes \mathcal{C}$, which is unitarily equivalent to the COSET pair representation, decomposes completely into tensor products, $\rho_{r}^{\mathcal{A}} \otimes \rho_{s}^{\mathcal{C}}$, of irreducible, localised representations $\rho_{r}^{\mathcal{A}}$ of $\mathcal{A}$ and $\rho_{s}^{\mathcal{C}}$ of $\mathcal{C}$, respectively:

$$
\rho \cong \bigoplus Z_{r s} \rho_{r}^{\mathcal{A}} \otimes \rho_{s}^{\mathcal{C}}
$$

Remark: Even for Coset pairs of finite index it is not clear whether they are automatically spatial. This is true under additional conditions [KLM01, lemma 27], which are known to be satisfied if one of $\mathcal{A}, \mathcal{C}$ is completely rational [KLM01, corollary 14].

Covariance of a representation $\rho_{r}^{\mathcal{A}}$ localised in some $I_{0} \Subset \mathrm{S}^{1}$ means that there are unitaries $z_{\rho_{r}^{\mathcal{A}}}(\tilde{g})$ implementing the equivalence of $\rho_{r}^{\mathcal{A}}$ and $\alpha_{\mathbf{p}(\tilde{g})} \circ \rho_{r}^{\mathcal{A}} \circ \alpha_{\mathbf{p}(\tilde{g})^{-1}}$ :

$$
A d_{z_{\rho_{r}^{A}}(\tilde{g})} \circ \rho_{r}^{\mathcal{A}}=\alpha_{\mathfrak{p}(\tilde{g})} \circ \rho_{r}^{\mathcal{A}} \circ \alpha_{\mathfrak{p}(\tilde{g})^{-1}} .
$$

The map $g \mapsto z_{\rho_{r}^{A}}(\tilde{g})$ may be chosen to be a localised $\alpha$-cocycle with values in the universal $C^{*}$-algebra $\mathcal{A}_{\text {uni }}$ such that:

i) $z_{\rho_{r}^{\mathcal{A}}}(\tilde{g}) \in \mathcal{A}\left(I_{0} \cup \mathrm{p}(\tilde{g}) I_{0}\right) e_{\mathcal{A}}$, for $\tilde{g} \in P S L(2, \mathbb{R})^{\sim}$ which are close to the identity and satisfy $I_{0} \cup \mathrm{p}(\tilde{g}) I_{0} \Subset \mathrm{S}^{1}$.

ii) $z_{\rho_{r}^{\mathcal{A}}}(\tilde{g} \tilde{h})=\alpha_{\mathbf{p}(\tilde{g})}\left(z_{\rho_{r}^{\mathcal{A}}}(\tilde{h})\right) z_{\rho_{r}^{\mathcal{A}}}(\tilde{g}), \tilde{g}, \tilde{h} \in P S L(2, \mathbb{R})^{\sim}$.

If $U_{\rho_{r}^{\mathcal{A}}}$ implements covariance in the representation $\rho_{r}^{\mathcal{A}}$, we have:

$$
z_{\rho_{r}^{\mathcal{A}}}(\tilde{g})=U(\mathrm{p}(\tilde{g})) e_{\mathcal{A}} U_{\rho_{r}^{\mathcal{A}}}(\tilde{g})^{*}, \tilde{g} \in P S L(2, \mathbb{R})^{\sim} .
$$

More details on localised covariance cocycles may be found in [Rob76], [GL92], [GL96], [Sch97, §27].

For $\tilde{g}$ as in i) one may identify $z_{\rho_{r}^{A}}(\tilde{g})$ with its representative in the inclusion $\mathcal{A} \subset \mathcal{B}$. By the very construction of the covariance cocycles $z_{\rho_{r}^{\mathcal{A}}}(\tilde{g})$ for general $\tilde{g}$ (see eg [Sch97, lemma 27.2]), it is clear that $z_{\rho_{r}^{\mathcal{A}}}(\tilde{g})$ may be identified with its 
representative in the inclusion $\mathcal{A} \subset \mathcal{B}$ as well: The inclusion defines a locally normal representation of $\mathcal{A} e_{\mathcal{A}}$ which lifts to a representation of $\mathcal{A}_{\text {uni }}$. The universal $C^{*}$-algebra, in turn, contains the covariance cocycle $z_{\rho_{r}^{\mathcal{A}}}(\tilde{g})$.

All that has been said about the $\rho_{r}^{\mathcal{A}}$ holds, mutatis mutandis, for the representations $\rho_{s}^{\mathcal{C}}$; these representations shall be localised in $J_{0} \Subset \mathrm{S}^{1}$.

We now choose complete sets of orthonormal intertwiners $\mathcal{A}\left(I_{0}\right) \ni w_{s}^{i}: \rho_{r}^{\mathcal{A}} \otimes$ $i d \rightarrow \rho$ and $\mathcal{C}\left(J_{0}\right) \ni w_{s}^{j}: i d \otimes \rho_{s}^{\mathcal{C}} \rightarrow \rho$ such that the $w_{r}^{i} w_{s}^{j}: \rho_{r}^{\mathcal{A}} \otimes \rho_{s}^{\mathcal{C}} \rightarrow \rho$ form a complete orthonormal set of intertwiners. These are intertwiners for all $\mathcal{A} \otimes \mathcal{C}(I \times J)$, if $I \times J \supset I_{0} \times J_{0}$ [GL96, proof of theorem 2.3].

Using the isometry $v$ from lemma $\mathrm{V} .7$ we define the charged intertwiners for our spatial Coset pair of finite index as:

$$
\psi_{r s}^{i j}:=w_{r}^{i *} w_{s}^{j *} v \in \mathcal{B}^{1+1}\left(I_{0} \times J_{0}\right) .
$$

These operators are charged intertwiners $i d \rightarrow \rho_{r}^{\mathcal{A}} \otimes \rho_{s}^{\mathcal{C}}$ for all $\mathcal{A} \otimes \mathcal{C}(I \times J)$, if $I \times$ $J \supset I_{0} \times J_{0}$. The map $w_{r}^{i} w_{s}^{j} \mapsto \psi_{r s}^{i j}$ defines an anti-isomorphism of the respective intertwiner spaces [LR95, lemma 4.5], [Reh94b, proposition 2]. Regarding the transformation behaviour of the charged intertwiners we have:

Lemma V.10: For $(\tilde{g}, \tilde{h}) \in \operatorname{PSL}(2, \mathbb{R})^{\sim} \times \operatorname{PSL}(2, \mathbb{R})^{\sim}$ holds:

$$
\alpha_{(\tilde{g}, \tilde{h})} \psi_{r s}^{i j}=A d_{U^{\mathcal{A}}(\tilde{g})} A d_{U_{\mathcal{A}^{\prime}(\tilde{h})}}\left(\psi_{r s}^{i j}\right)=z_{\rho_{r}^{\mathcal{A}}}(\tilde{g}) z_{\rho_{s}^{\mathcal{C}}}(\tilde{h}) \psi_{r s}^{i j} .
$$

Proof: The method of proof is standard [Reh94b, lemma (3.6)],[Lon01, corollary 19]. We look at some neighbourhood $\mathcal{U}$ of the identity in $\operatorname{PSL}(2, \mathbb{R})^{\sim} \times P S L(2, \mathbb{R})^{\sim}$. It is easy to show on grounds of the covariance of the endomorphisms $\rho_{r}^{\mathcal{A}}, \rho_{s}^{\mathcal{C}}$ (see equation V.21) that the following mapping, defined for $(\tilde{g}, \tilde{h}) \in \mathcal{U}$, leaves globally invariant the spaces of charged intertwiners:

$$
\left((\tilde{g}, \tilde{h}), \psi_{r s}^{i j}\right) \mapsto z_{\rho_{r}^{\mathcal{A}}}(\tilde{g})^{*} z_{\rho_{s}^{\mathcal{C}}}(\tilde{h})^{*} \alpha_{(\tilde{g}, \tilde{h})}\left(\psi_{r s}^{i j}\right) .
$$

Indeed, this map defines a unitary representation of $P S L(2, \mathbb{R})^{\sim} \times P S L(2, \mathbb{R})^{\sim}$ on the finite-dimensional HILBERT spaces of charged intertwiners. This representation has to be trivial, as there are no non-trivial unitary, finite-dimensional representations of this group.

Hence, the transformation behaviour of $b \in \mathcal{B}^{1+1}(\mathcal{O}), \mathcal{O} \supset I_{0} \times J_{0}$ has the following form:

$$
\alpha_{(\tilde{g}, \tilde{h})}(b)=\sum_{i, j, r, s}[\mathcal{B}: \mathcal{A} \subseteq \mathcal{C}] \alpha_{(\tilde{g}, \tilde{h})}\left(\mu_{\mathcal{O}}\left(b \psi_{r s}^{i j *}\right)\right) z_{\rho_{r}^{\mathcal{A}}}(\tilde{g}) z_{\rho_{s}^{\mathcal{C}}}(\tilde{h}) \psi_{r s}^{i j}
$$

For chiral rotations by $2 \pi$ we have in terms of the lower bound $h_{r}^{\mathcal{A}}$ of the spectrum of the conformal Hamiltonian $L_{0}$ in the representation $\rho_{r}^{\mathcal{A}}$ [GL96]: $z_{\rho_{r}^{\mathcal{A}}}(\tilde{R}(2 \pi))=e^{-i 2 \pi h_{r}^{\mathcal{A}}}$. This proves, that the adjoint action of $\operatorname{Ad}_{U_{\mathcal{A}} \mathcal{A}(\tilde{R}(2 \pi))}$ on any $\mathcal{B}^{1+1}(\mathcal{O}), \mathcal{O} \supset I_{0} \times J_{0}$, defines an automorphism of this algebra. We state this result explicitly for the chiral situation: 
Proposition V.11: Let $\mathcal{A} \subseteq \mathcal{C} \subset \mathcal{B}$ be a spatial COSET pair with finite index and assume $U^{\mathcal{A}}$ to have the net-endomorphism property. Then $A d_{U^{\mathcal{A}}(\tilde{R}(2 \pi))}(\mathcal{B}(I))=$ $\mathcal{B}(I)$ for all $I \Subset \mathrm{S}^{1}$.

Proof: First, one goes into the chirally holographic picture, then one constructs the representation $\rho$ unitarily equivalent to $\mathcal{A} \subset \mathcal{C} \subset \mathcal{B}^{1+1}$ and localised in $I \times I$, and one proceeds as above until one arrives at (V.25). Now the statement is obvious, if one restricts attention to the time-zero algebra $\mathcal{B}^{1+1}(I \times I)=\mathcal{B}(I)$.

Hence we improved proposition IV.6 on the net-endomorphic action of $U^{\mathcal{A}}$ on $\mathcal{B}$ under additional assumptions. We expect this automorphic action of $A d_{U^{\mathcal{A}}(\tilde{R}(2 \pi))}$ on each $\mathcal{B}(I), I \Subset \mathrm{S}^{1}$, in general.

We continue with an application: the spectrum of $U^{\mathcal{A}}(\tilde{R}(2 \pi))$ is determined (almost completely), provided $U^{\mathcal{A}}$ has sharp geometrical action. The argument applies to the corresponding situation of chiral observables in any $1+1$ dimensional conformal theory as well.

Proposition V.12: Let $\mathcal{A} \subseteq \mathcal{C} \subset \mathcal{B}$ be a spatial Coset pair with finite index and assume $U^{\mathcal{A}}$ to have sharp geometrical action on $\mathcal{B}$. Then we have $\operatorname{Spec}\left(U^{\mathcal{A}}(\tilde{R}(2 \pi))\right) \subset\{ \pm 1\}$, and each $b \in \mathcal{B}(I), I \Subset \mathrm{S}^{1}$, has a decomposition:

$$
b=b_{+}+b_{-}, b_{ \pm} \in \mathcal{B}(I), A d_{U^{\mathcal{A}}(\tilde{R}(2 \pi))}\left(b_{ \pm}\right)= \pm b_{ \pm} .
$$

Proof: We analyse this problem in the holographic picture in $1+1$ dimensions (see section IV ,2). The DHR endomorphism $\rho_{r}^{\mathcal{A}} \otimes \rho_{s}^{\mathcal{C}}$ has four statistics operators: left and right statistics operators for spacelike directions, denoted $\varepsilon_{r s}^{>}$and $\varepsilon_{r s}^{<}$, and upper and lower statistics operators for time-like directions, which we write as $\varepsilon_{r s}^{\vee}$ and $\varepsilon_{r s}^{\wedge}$. All arguments on statistics have nothing to do with positivity of energy, but only with geometry and commutativity.

For the chiral endomorphisms there is a right and a left statistics operator, namely $\varepsilon_{r}^{+}$and $\varepsilon_{r}^{-}$for $\rho_{r}^{\mathcal{A}}$ and correspondingly $\varepsilon_{s}^{+}$and $\varepsilon_{s}^{-}$for $\rho_{s}^{\mathcal{C}}$. We have:

$$
\varepsilon_{r s}^{<}=\varepsilon_{r}^{+} \otimes \varepsilon_{s}^{+}, \varepsilon_{r s}^{\vee}=\varepsilon_{r}^{+} \otimes \varepsilon_{s}^{-} .
$$

According to the general analysis of ReHRen [Reh94a, proposition 4], [Reh01, lemma 3.1] the corresponding statistical phases satisfy:

$$
1=\kappa_{r s}^{<}=\kappa_{r}^{+} \kappa_{s}^{+}, 1=\kappa_{r s}^{\vee}=\kappa_{r}^{+} \kappa_{s}^{-} .
$$

The left identity follows from locality (commutativity for spacelike separation) and the right one from commutativity for timelike separation.

The left and right statistics operators are connected by the identity $\varepsilon_{r, s}^{+}=\varepsilon_{r, s}^{-*}$, which yields directly $\kappa_{r, s}^{+}=\overline{\kappa_{r, s}^{-}}$. Combining this with the conformal spin and statistics theorem [GL96] and equations (V.28) leads to

$$
\kappa_{r}^{+2}=\left(e^{i 2 \pi h_{r}^{\mathcal{A}}}\right)^{2}=1 \Leftrightarrow h_{r}^{\mathcal{A}} \in \frac{1}{2} \mathbb{N} .
$$


The remainder follows from the decomposition of local observables and the transformation law of charged intertwiners, see equation (V.25).

If the decomposition of the vacuum representation of a chiral conformal theory $\mathcal{B}$ considered as representation of a spatial Coset pair $\mathcal{A} \subset \mathcal{C}$ of finite index is known and $U^{\mathcal{A}}$ has the net-endomorphism property, it is very simple to check whether the Borchers-SugAwARA representation $U^{\mathcal{A}}$ can have sharp geometrical action by means of proposition V.12. In most examples sharp geometrical action and hence time-like commutativity of the quasi-theory in $1+1$ dimensions can be ruled out this way. In section 3 some typical branchings for current subalgebras and their Coset models are stated. Obviously, tensor products of chiral conformal theories and tensor products of chiral fermions (analogous to bounded Bose fields) yield chiral subnets with sharp geometrical action. It is unclear whether there are other examples of this structure; compare remarks at the end of section IV.2.

The result of the spectrum of chiral conformal rotations by $2 \pi$ in proposition V.12 is familiar in the context of conformal quantum field theory in terms of operator-valued distributions. Here, the two-point function of a quasi-primary field $\Phi_{d_{+} d_{-}}$of chiral scaling dimensions $d_{+}$and $d_{-}$is determined by the transformation behaviour of the field to be:

$$
\left\langle\Omega, \Phi_{d_{+} d_{-}}\left(x_{+}, x_{-}\right) \Phi_{d_{+} d_{-}}\left(y_{+}, y_{-}\right) \Omega\right\rangle=C_{\Phi}\left(\frac{-i}{\Delta_{+}+i \varepsilon}\right)^{2 d_{+}}\left(\frac{-i}{\Delta_{-}+i \varepsilon}\right)^{2 d_{-}}
$$

Here, $C_{\Phi}$ is some positive constant and $\Delta_{+}=x_{+}-y_{+}, \Delta_{-}=x_{-}-y_{-}$.

Commutativity of the field for spacelike, $\Delta_{+} \Delta_{-}<0$, and for time-like separation, $\Delta_{+} \Delta_{-}>0$, yields a symmetry of the two-point function in (V.30) with respect to $\Delta_{ \pm} \leftrightarrow-\Delta_{ \pm}$. Taking this together with the analyticity properties of the distributions on the right-hand side of (V.30) results in restrictions on the possible values of $d_{ \pm}$:

$$
\left(\frac{-i}{\Delta+i \varepsilon}\right)^{2 d}=e^{ \pm i 2 \pi d}\left(\frac{-i}{-\Delta+i \varepsilon}\right)^{2 d}, \quad \Delta \gtrless 0 .
$$

Commutativity in spacelike directions results in $e^{i 2 \pi\left(d_{+}-d_{-}\right)}=1$ and commutativity for timelike directions requires $e^{i 2 \pi\left(d_{+}+d_{-}\right)}=1$. Both together imply $d_{ \pm} \in \frac{1}{2} \mathbb{N}$ and hence the spectrum of chiral rotations by $2 \pi$ is found again to be contained in $\{ \pm 1\}$. 


\section{Coset construction of normal CTPS}

The main goal in this section is to get examples of normal canonical tensor product subfactors (normal CTPS). This concept was introduced by REHREN [Reh00] in the context of inclusions of tensor products of chiral observables in a 1+1-dimensional conformal theory. According to the definition of normal CTPS [Reh00], Coset pairs form another class of examples of this structure, if they are spatial, normal and of finite index. Provided the net-endomorphism property holds for the respective inner-implementing representation, such CosET pairs have an interpretation directly analogous to that originally considered in [Reh00] (by chiral holography, section IV,2).

In the examples which we discuss below there is a Coset pair $\mathcal{A} \subset \mathcal{C} \subset \mathcal{B}$ which is spatial, the decomposition (branching rules) of this representation of $\mathcal{A} \otimes \mathcal{C}$ available from group-theoretic investigations. The branchings for $\mathcal{A} \subset \mathcal{C} \subset$ $\mathcal{B}$ are finite and, moreover, both $\mathcal{A}$ and $\mathcal{C}$ are completely rational, a property which consists of three parts: the models satisfy the split property and strong additivity (cf section II,1.3) and their respective $\mu$-index is finite. The $\mu$-index is the index of the inclusion described in equation (IV.10), and finite $\mu$-index implies in particular that there are only finitely many sectors which all have finite statistical dimension [KLM01, theorem 33]. For this reason the branching tells us that the Coset pair $\mathcal{A} \subset \mathcal{C} \subset \mathcal{B}$ has finite index.

Because of strong additivity of $\mathcal{A}$ and $\mathcal{C}$ the Coset pair $\mathcal{A}_{\max } \subseteq \mathcal{C}_{\max } \subset \mathcal{B}$ is known to be normal, but the same follows from the presence of stress-energy tensors in both subnets. We will use our knowledge on $\mathcal{A}, \mathcal{C}$ and on $\mathcal{A} \subset \mathcal{C} \subset \mathcal{B}$ in order to obtain the branching rules for $\mathcal{A}_{\max } \subset \mathcal{C}_{\max } \subset \mathcal{B}$.

Lemma V.13: Assume $\mathcal{A} \subseteq \mathcal{C} \subset \mathcal{B}$ is a spatial Coset pair of finite index. Then $\mathcal{A}_{\max } @ \mathcal{C}_{\max } \subset \mathcal{B}$ is spatial and of finite index as well.

Proof: The statement on the index follows from an identity contained in [Lon90], compare the argument in the proof of lemma V.1.

By assumption, $\mathcal{A} \subset \mathcal{B}$ is unitarily equivalent to a direct sum of irreducible representations of $\mathcal{A}$, the representation space decomposing as $\mathscr{H}=\sum_{i} \mathscr{H}_{(i)} \otimes$ $\mathscr{H}_{i}$, where the $\mathscr{H}_{i}$ are the representation spaces of inequivalent, irreducible representations $\pi_{i}$ and the $\mathscr{H}_{(i)}$ are multiplicity spaces. We denote the projection onto $\mathscr{H}_{(i)} \otimes \mathscr{H}_{i}$ by $P_{i}$.

For an operator $V$ which commutes with all of $\mathcal{A}$, the operators $P_{i} V P_{j}$ define intertwiners from $\mathbb{1}_{(j)} \otimes \pi_{j}$ to $\mathbb{1}_{(i)} \otimes \pi_{i}$, which by inequivalence of the $\pi_{i}$ implies: $P_{i} V P_{j}=\delta_{i j} P_{i} V P_{i}$, ie $V=\sum_{i} P_{i} V P_{i}$. Obviously, $P_{i} V P_{i}$ is of the form $P_{i} V P_{i}=$ : $V_{(i)} \otimes \mathbb{1}_{i}$, and this means that the multiplicity spaces $\mathscr{H}_{(i)}$ form representation spaces of $\mathcal{C}_{\text {max }}$. These representations are completely reducible into irreducibles by finiteness of index [GL96]. The same argument works for $\mathcal{A}_{\max }$ as well. 
For definiteness the following proposition summarises the relevant results on spatial Coset pairs of finite index obtained so far:

Proposition V.14: $\mathcal{A} \subset \mathcal{C} \subset \mathcal{B}$ a spatial Coset pair with finite index. Assume $U^{\mathcal{A}}, U^{\mathcal{C}}$ to have the net-endomorphism property.

Then $\mathcal{A}_{\text {max }} \subseteq \mathcal{C}_{\max } \subset \mathcal{B}$ is a normal, irreducible, spatial Coset pair with finite index, and thus forms a normal CTPS.

Proof: The statement was proven with proposition V.2 and lemma V.13.

The branchings of the Coset pairs $\mathcal{A} \subset \mathcal{C} \subset \mathcal{B}$ and $\mathcal{A}_{\max }\left(\mathcal{C}_{\max } \subset \mathcal{B}\right.$ are written as follows:

$$
\begin{aligned}
\rho & \cong \bigoplus Z_{r s} \rho_{r}^{\mathcal{A}} \otimes \rho_{s}^{\mathcal{C}} \\
\rho_{\max } & \cong \bigoplus Z_{u v}^{\max } \rho_{u}^{\mathcal{A}_{\max }} \otimes \rho_{v}^{\mathcal{C}_{\max }}
\end{aligned}
$$

The finite matrices $Z_{r s}, Z_{u v}^{\max }$ are called the coupling matrix of the respective decomposition. Normality of a CTPS is equivalent to $Z_{0 s}=\delta_{0 s}, Z_{r 0}=\delta_{r 0}$ [Reh00, corollary 3.5].

The probably most remarkable result of [Reh00], translated in our setting, is the following: If $\mathcal{A}_{\max } \subset \mathcal{C}_{\max } \subset \mathcal{B}$ forms a normal CTPS, then the sets of superselection sectors $\left[\rho_{u}^{\mathcal{A}_{\text {max }}}\right],\left[\rho_{v}^{\mathcal{C}_{\text {max }}}\right]$ appearing in $(\mathrm{V} .33)$ are invariant under conjugation and their direct sums span a subsystem of sectors which is closed under fusion. The coupling matrix $Z_{u v}^{\max }$ is in fact a permutation matrix, ie for given $u$ there is exactly one $v$ for which $Z_{u v}^{\max } \neq 0$ and the map $u \mapsto v(u)$ derived from this condition yields $Z_{u v}^{\max }=\delta_{u v(u)}$. In this way the coupling matrix induces an isomorphism of fusion algebras for the subsystems of sectors of $\mathcal{A}_{\text {max }}$, $\mathcal{C}_{\max }$ generated by the subsectors of $\rho_{\max }$ through $\left[\rho_{u}^{\mathcal{A}_{\max }}\right] \mapsto\left[\rho_{v(u)}^{\mathcal{C}_{\max }}\right]$. This means in particular that the statistical dimensions of coupled sectors have to coincide: $d\left(\rho_{u}^{\mathcal{A}_{\max }}\right)=d\left(\rho_{v(u)}^{\mathcal{C}_{\max }}\right)$.

MÜGER has announced an extension of these findings in case $\mathcal{B}$ has trivial superselection structure, ie all locally normal representations of $\mathcal{B}$ are equivalent to the vacuum representation [Müg02]. In this case the isomorphism of fusion rules extends to an isomorphism of the respective DHR subcategories. These claims motivated our discussing the example contained in section 3.3 .

The results of REHREN and MÜGER show that branchings of COSET pairs establish direct links between the superselection structure of $\mathcal{A}_{\text {max }}$ and $\mathcal{C}_{\text {max }}$. Moreover, the DHR triple of a chiral subnet of finite index determines completely the vacuum representation of the larger theory and, in the opposite direction, the embedding itself determines the vacuum representation of the subnet. In principle, the superselection structures of $\mathcal{A}, \mathcal{C}, \mathcal{A}_{\text {max }}, \mathcal{C}_{\text {max }}$ all are connected by embedding, local extension and coupling, provided the assumptions of proposition V.14 hold (or, possibly, alterations of these). 
Of these connections we use only rudimentary details. Let $\iota^{\mathcal{A}}: \mathcal{A}(I) \hookrightarrow$ $\mathcal{A}_{\text {max }}(I)$ denote the embedding inducing the inclusion $\mathcal{A}(I) \subset \mathcal{A}_{\text {max }}(I)$; this inclusion is irreducible by the assumption of finite index (lemma V.1). There is an injective homomorphism $\bar{\iota}^{\mathcal{A}}: \mathcal{A}_{\text {max }}(I) \rightarrow \mathcal{A}(I)$ such that $\iota^{\mathcal{A}} \bar{\iota}^{\mathcal{A}}$ equals $\gamma_{I}^{\mathcal{A}}$, the canonical endomorphism of $\mathcal{A}(I) \subset \mathcal{A}_{\text {max }}(I)$, and we have $\bar{\iota}^{\mathcal{A}} \iota^{\mathcal{A}}=\rho_{I}^{\mathcal{A}}$ for the dual canonical endomorphism. An endomorphism $\sigma$ of $\mathcal{A}_{\max }(I)$ possesses $\bar{\iota}^{\mathcal{A}} \sigma \iota^{\mathcal{A}}$ as restriction of $\sigma$ to an endomorphism of $\mathcal{A}(I)$. For a short summary on these notions see, for example, [LR95, section 2.8.C.]. Since the decompositions of $\rho_{\max }, \rho^{\mathcal{A}}$ as localised representations and as dual canonical endomorphisms are the same (as covariant representations of finite index [GL96, Lon01]), we may study the fusion rules of restricted representations by looking at the corresponding restricted endomorphisms.

In the examples below, the fusion rules of restricted endomorphisms are known, while their decomposition into irreducibles is not (initially). But we do know the following: The canonical endomorphism ${ }^{1} \gamma_{I}^{\mathcal{A}}$ contains the identity on $\mathcal{A}_{\max }(I)$ with multiplicity 1 . Since the dual canonical endomorphism $\rho_{\max }$ is self-conjugate, to every $\rho_{u}^{\mathcal{A}_{\text {max }}}$ appearing in (V.33) the conjugate $\rho_{\bar{u}}^{\mathcal{A}_{\text {max }}}$ appears as well. The product of the corresponding restricted endomorphisms must contain $\rho^{\mathcal{A}}$ :

$$
\begin{aligned}
\bar{\iota}^{\mathcal{A}} \rho_{I u}^{\mathcal{A}_{\max }} \iota^{\mathcal{A}} \bar{\iota}^{\mathcal{A}} \rho_{I \bar{u}}^{\mathcal{A}_{\max }} \iota^{\mathcal{A}} & =\bar{\iota}^{\mathcal{A}} \rho_{I u}^{\mathcal{A}_{\max }} \gamma_{I}^{\mathcal{A}} \rho_{I \bar{u}}^{\mathcal{A}_{\max }} \iota^{\mathcal{A}} \\
& \succ \bar{\iota}^{\mathcal{A}} \rho_{I u}^{\mathcal{A}_{\text {max }}} i d^{\mathcal{A}_{\max }(I)} \rho_{I \bar{u}}^{\mathcal{A}_{\text {max }}} \iota^{\mathcal{A}} \\
& \succ \bar{\iota}^{\mathcal{A}} i d^{\mathcal{A}_{\max }(I)} \iota^{\mathcal{A}}=\rho_{I}^{\mathcal{A}}
\end{aligned}
$$

The same applies to $\mathcal{C}$ and $\mathcal{C}_{\text {max }}$, of course.

Below we will derive the branching of the extended Coset pairs $\mathcal{A}_{\max }\left(\mathrm{C} \mathcal{C}_{\max } \subset\right.$ $\mathcal{B}$, which define normal CTPS. The basis for the discussions are branching formulae for a Coset pair $\mathcal{A} \subset \mathcal{C} \subset \mathcal{B}$ which were derived by character arguments in [GKO86], [KW88], [KNS88]. These (and other) sources contain branchings for a number of other COSET pairs as well. We have selected the three examples discussed in sections $3.1,3.2,3.3$ for their special interest.

The main tools of the arguments below are the present knowledge on current algebras and of $\mathcal{V} i r_{c<1}$ models, the spatial identification of $\mathcal{A}_{\text {max }}$ and $\mathcal{C}_{\text {max }}$ (proposition IV.8), the results on normal CTPS discussed above [Reh00] and some general results on completely rational models, mainly from [KLM01].

\subsection{The Coset pairs of Goddard, Kent and Olive}

One achievement in 1+1-dimensional and chiral conformal quantum field theory was the construction of the stress-energy tensors with central charge less than 1

\footnotetext{
${ }^{1}$ It has to be emphasised that the canonical endomorphism does in general not have a continuation to a DHR endomorphism and that thus not all irreducibles contained in it need to have an interpretation as a DHR endomorphism. In this respect the canonical endomorphism is quite different from the dual canonical endomorphism.
} 
as Coset models by Goddard, Kent and Olive (GKO) [GKO85, GKO86]. The analysis of FrIEDAN, QIU AND SHEnkER [FQS84, FQS85a, FQS86] (cf [Lan88]) had shown that below 1 the central charges had to be contained in the discrete series:

$$
c(m)=1-\frac{6}{(m+2)(m+3)}, \quad m \in \mathbb{N} .
$$

The Coset construction of GKO proved these models to exist as CoseT models associated with the chiral subnet $\mathcal{L} S U(2)_{m+1} \subset \mathcal{L} S U(2)_{1} \otimes \mathcal{L} S U(2)_{m}$, the inclusion induced by the diagonal embedding of the colour algebras. The Coset stress-energy tensor, which generates the $\mathcal{V} i r_{c(m)}$ model, is simply given by the difference of the SUGAWARA stress-energy tensors of $\mathcal{L} S U(2)_{1} \otimes \mathcal{L} S U(2)_{m}$ and of $\mathcal{L} S U(2)_{m+1}$. There are various other ways to construct $\mathcal{V} i r_{c<1}$ models as Coset stress-energy tensors of current algebra inclusions (classification: [BG87], examples eg in [GKO86, KW88]).

The branching of the vacuum representation of $\mathcal{L} S U(2)_{1} \otimes \mathcal{L} S U(2)_{m}$ with respect to the Coset pair $\mathcal{L} S U(2)_{m+1}$ C $\mathcal{V} i r_{c(m)}$ reads [GKO86], [KW88, 4.1.a]:

$$
L\left(\Lambda_{0}\right) \otimes L\left(m \Lambda_{0}\right) \uparrow \cong \sum_{l: 0 \leq 2 l \leq m+1} L\left((m+1-2 l) \Lambda_{0}, 2 l \Lambda_{1}\right) \otimes V_{1,2 l+1}^{(m)}
$$

By the symbol $\uparrow$ we indicate that the formula gives the decomposition of $L\left(\Lambda_{0}\right) \otimes$ $L\left(m \Lambda_{0}\right)$ as a representation of $\mathcal{L} S U(2)_{m+1} \otimes \mathcal{V} i r_{c(m)}$.

The highest-weight representations of $\mathcal{L} S U(2)_{k}$ are denoted as in [KW88] according to the classification of (unitarisable) highest-weight representations of affine KAC-Moody algebras (see [Kac90], summary in [KW88]). $L\left(k \Lambda_{0}\right)$ stands for the vacuum representation of $\mathcal{L} S U(2)_{k}$ and $L\left((k-l) \Lambda_{0}, l \Lambda_{1}\right)$ stands for the highest-weight representation of $\mathcal{L} S U(2)_{k}$ in which the horizontal subalgebra acts as a spin $l / 2$ representation of $S U(2)$ on the vectors of lowest energy.

The highest-weight vector of the representation $L\left((k-l) \Lambda_{0}, l \Lambda_{1}\right)$ has conformal energy $l(l+2) /(4(2+k))$. Hence, not all the lowest conformal energy eigenvalues occurring in (V.36) can be half-integers; this prohibits sharp geometrical action of the inner-implementing representation $U^{\mathcal{L} S U(2)_{m+1}}$ (proposition V.12).

The highest-weight representations $V_{r, s}^{(m)}$ of $\mathcal{V}_{i r_{c(m)}}$ are identified by the energy $h_{r, s}^{(m)}$ of their lowest-energy vector. There are only finitely many of such representations, namely:

$$
h_{r, s}^{(m)}=\frac{[(m+3) r-s(m+2)]^{2}-1}{4(m+2)(m+3)}, \quad 1 \leq s \leq r \leq m+1 .
$$

Together with ${ }^{2}$ proposition IV.8 equation (V.36) shows that $\mathcal{V}_{i(m)}$ is indeed the maximal CosET model; since there are no chiral subnets in a net generated

\footnotetext{
${ }^{2}$ Alternatively one may argue as in [KL02, lemma 3.2, corollary 3.3]
} 
by a stress-energy tensor [Car98], these Coset models are minimal among the non-trivial ones as well.

$\mathrm{XU}$ [Xu00a, Xu99, Xu01] studied the Coset models of the chiral subnets $\mathcal{L} S U(n)_{k+l} \subset \mathcal{L} S U(n)_{k} \otimes \mathcal{L} S U(n)_{l}$ arising from the diagonal embedding of colour algebras. His results cover, of course, the GKO Cosets ${ }^{3}$. KaWAHIGASHI AND LONGO [KL02] completed the proof of the $\mathcal{V} i r_{c<1}$ models being completely rational. Since the $\mathcal{L} S U(n)_{k}$ are completely rational as well [GF93, TL97, Xu00b], this tells us that $\mathcal{L} S U(2)_{m+1} \subset \mathcal{V} i r_{c(m)} \subset \mathcal{L} S U(2)_{1} \otimes \mathcal{L} S U(2)_{m}$ does indeed form a normal CTPS.

Furthermore, KawAHIGASHI AND LONGO [KL02] completed the proof of the $\mathcal{V} i r_{c(m)}$ fusion rules being identical with the ones expected from operator product expansions of primary fields and the statistical representation of $S L(2, \mathbb{Z})$ [Reh90] being identical with the modular representation (see eg [DFMS96, section 10.6]). The latter identity is induced by the diagonalisation of the fusion rules by both representations (VERLINDE formulae, [Ver88] [Reh90]) and the identification of the highest weights $h_{r, s}^{(m)}$. More details on the problem of matching these two representations of $S L(2, \mathbb{Z})$ for general rational models in [Reh01], [Fuc94].

For future reference we state the fusion rules

$$
\phi_{r_{1}, s_{1}}^{(m)} \phi_{r_{2}, s_{2}}^{(m)} \cong \bigoplus_{\substack{r=\left|r_{1}-r_{2}\right|+1, r+r_{1}+r_{2}: \text { odd } \\ s=\left|s_{1}-s_{2}\right|+1, s+s_{1}+s_{2} \text { :odd }}}^{r_{\max }} \oint_{r, s}^{(m)},
$$

where $r_{\max }=\min \left(r_{1}+r_{2}-1,2 m+3-r_{1}-r_{2}\right), s_{\max }=\min \left(s_{1}+s_{2}-1,2 m+5-\right.$ $\left.s_{1}-s_{2}\right)$. It easy to see that all $\phi_{r, s}^{(m)}$ are self-conjugate, ie the vacuum sector $\phi_{1,1}^{(m)}$ is contained only in the squares $\left(\phi_{r, s}^{(m)}\right)^{2}$ and it appears in these precisely once. The functions on the right-hand side of (V.37) are invariant with respect to the simultaneous replacement $r \rightarrow m+2-r, s \rightarrow m+3-s$. This identification has to be taken into account when calculating the fusion rules.

The statistical dimensions of the representations $\phi_{r, s}^{(m)}$ are:

$$
d_{r, s}^{(m)}=\frac{\sin (r \pi /(m+2))}{\sin (\pi /(m+2))} \frac{\sin (s \pi /(m+3))}{\sin (\pi /(m+3))} .
$$

\subsection{Extension $\left(A_{10}, E_{6}\right)$ of $\mathcal{V}_{\frac{21}{22}}$ as a COSET model}

KAWAHIGASHi AND LONGO [KL02] completed the classification of local extensions of the $\mathcal{V} i r_{c<1}$ models. Most of these are given as orbifolds: the local extension contains the $\mathcal{V} i r_{c<1}$ model as a fixed-point subtheory with respect to a $\mathbb{Z}_{2}$ symmetry; some of these are among the maximal Coset models associated with current subalgebras, as branchings contained in [KW88] show. Only four local extensions are of a different type (exceptional cases). For two

\footnotetext{
${ }^{3}$ For an earlier investigation on the $\mathcal{V} i r_{c<1}$-models as GKO CosET models see [Lok94].
} 
of these KAWAHIGASHI AND LONGO [KL02] gave a rigorous interpretation as COsET models of current subalgebras following suggestions of BöCKENHAUER AND Evans [BE99]. These are the extension $\left(E_{6}, A_{12}\right)$ of $\mathcal{V} i r_{c(10)}$, associated with $\mathcal{L} S U(2)_{11} \subset \mathcal{L} S O(5)_{1} \otimes \mathcal{L} S U(2)_{1}$, and the extension $\left(E_{8}, A_{30}\right)$ of $\mathcal{V}_{i r}(28)$, associated with $\mathcal{L} S U(2)_{29} \subset \mathcal{L} G(2)_{1} \otimes \mathcal{L} S U(2)_{1}$.

A third of the exceptional local extensions is identified through the branching rules for the Coset pair $\mathcal{L} S U(9)_{2} \subset \mathcal{V} i r_{c(9)} \subset \mathcal{L} E(8)_{2}[\mathrm{KW} 88,4.3 . \mathrm{a}]$ :

$$
L\left(2 \Lambda_{0}\right)\left\lceil\cong \sum_{l=0}^{4}\left[L\left(\mu_{2 l+1}\right)+L\left(\sigma \mu_{2 l+1}\right)+L\left(\sigma^{2} \mu_{2 l+1}\right)\right] \otimes\left[V_{2 l+1,1}^{(9)}+V_{2 l+1,7}^{(9)}\right]\right.
$$

By the symbol $\uparrow$ we indicate that the formula gives the decomposition of $L\left(2 \Lambda_{0}\right)$ as a representation of $\mathcal{L} S U(9)_{2} \otimes \mathcal{V} i r_{c(9)}$.

We have in (V.40):

\begin{tabular}{r|ccccc}
$l$ & 0 & 1 & 2 & 3 & 4 \\
\hline$\mu_{2 l+1}$ & $2 \dot{\Lambda}_{0}$ & $\dot{\Lambda}_{1}+\dot{\Lambda}_{8}$ & $\dot{\Lambda}_{2}+\dot{\Lambda}_{7}$ & $\dot{\Lambda}_{3}+\dot{\Lambda}_{6}$ & $\dot{\Lambda}_{4}+\dot{\Lambda}_{5}$ \\
$\sigma \mu_{2 l+1}$ & $2 \dot{\Lambda}_{3}$ & $\dot{\Lambda}_{4}+\dot{\Lambda}_{2}$ & $\dot{\Lambda}_{5}+\dot{\Lambda}_{1}$ & $\dot{\Lambda}_{6}+\dot{\Lambda}_{0}$ & $\dot{\Lambda}_{7}+\dot{\Lambda}_{8}$ \\
$\sigma^{2} \mu_{2 l+1}$ & $2 \dot{\Lambda}_{6}$ & $\dot{\Lambda}_{7}+\dot{\Lambda}_{5}$ & $\dot{\Lambda}_{8}+\dot{\Lambda}_{4}$ & $\dot{\Lambda}_{0}+\dot{\Lambda}_{3}$ & $\dot{\Lambda}_{1}+\dot{\Lambda}_{2}$
\end{tabular}

As in [KW88] we denote the fundamental weights of the affine KAC-Moody algebra with horizontal subalgebra isomorphic to the LIE algebra of $S U(9)$ as $\dot{\Lambda}_{i}$, $i=0, \ldots, 8$. If one is interested in the highest weight of the module of $S U(9)$ formed by the vectors of lowest energy, one simply ignores the multiple of $\dot{\Lambda}_{0}$ and takes $\dot{\Lambda}_{i}, i=1, \ldots, 8$, to stand for the corresponding fundamental weights of $S U(9)$. The notation for the representations of the $\mathcal{V} i r_{c<1}$ models was introduced in the previous section already.

The conformal energies of the highest-weight vectors for $\mathcal{L} S U(9)_{2}$ occurring in (V.40) are given by:

\begin{tabular}{c|ccccc}
$l$ & 0 & 1 & 2 & 3 & 4 \\
\hline$h_{\mu_{2 l+1}}$ & 0 & $\frac{9}{11}$ & $\frac{16}{11}$ & $\frac{21}{11}$ & $\frac{24}{11}$ \\
$h_{\sigma \mu_{2 l+1}}$ & 2 & $\frac{20}{11}$ & $\frac{16}{11}$ & $\frac{10}{11}$ & $\frac{13}{11}$ \\
$h_{\sigma^{2} \mu_{2 l+1}}$ & 2 & $\frac{20}{11}$ & $\frac{16}{11}$ & $\frac{10}{11}$ & $\frac{13}{11}$
\end{tabular}

Most of these lowest conformal energy eigenvalues are not half-integers; this prohibits sharp geometrical action of the inner-implementing representation $U^{\mathcal{L S U}(9)_{2}}$ (proposition V.12).

Applying results of LoNGo [Lon01], we readily extract from (V.40) that $\mathcal{L} E(8)_{2}$ is completely rational, since $\mathcal{L} S U(9)_{2}$ [GF93, TL97, Xu00b] and $\mathcal{V} i r_{c(9)}$ [KL02] are and the branching is finite. The more interesting consequence of (V.40) is: 
Proposition V.15: The extension $\left(A_{10}, E_{6}\right)$ of $\mathcal{V}_{r_{c(9)}}$ is the maximal CoseT model associated with the chiral subnet $\mathcal{L} S U(9)_{2} \subset \mathcal{L} E(8)_{2}$.

The decomposition of $\rho_{\max }$, the localised representation of the COSET pair $\mathcal{A}_{\text {max }} \bigcirc \mathcal{C}_{\text {max }} \subset \mathcal{L} E(8)_{2}$ associated with $\mathcal{L} S U(9)_{2} \subset \mathcal{V} i r_{c(9)} \subset \mathcal{L} E(8)_{2}$, is:

$$
\rho_{\max } \cong \bigoplus_{l=0}^{4} \rho_{l}^{\mathcal{A}_{\max }} \otimes \rho_{l}^{\mathcal{C}_{\max }}
$$

where we have the following branchings:

$$
\begin{aligned}
& \bar{\iota}^{\mathcal{A}} \rho_{l}^{\mathcal{A}_{\max }} \iota^{\mathcal{A}} \cong \alpha_{L\left(\mu_{2 l+1}\right)} \oplus \alpha_{L\left(\sigma \mu_{2 l+1}\right)} \oplus \alpha_{L\left(\sigma^{2} \mu_{2 l+1}\right)}, \\
& \bar{\iota}^{\mathcal{C}} \rho_{l}^{\mathcal{C}_{\max }} \iota^{\mathcal{C}} \cong \phi_{2 l+1,1}^{(9)} \oplus \phi_{2 l+1,7}^{(9)} .
\end{aligned}
$$

The representations $\rho_{l}^{\mathcal{A}_{\max }}, \rho_{l}^{\mathcal{C}_{\max }}$ are all self-conjugate.

Proof: Following proposition IV.8 we read off (V.40) that the maximal CosET model $\mathcal{C}_{\text {max }}$ associated with $\mathcal{L} S U(9)_{2} \subset \mathcal{L} E(8)_{2}$ is a non-trivial local extension of $\mathcal{V} i r_{c(9)}$. The decomposition of the vacuum representation of $\mathcal{C}_{\text {max }}$ with respect to $\mathcal{V} i r_{c(9)}$ is given by:

$$
\rho^{\mathcal{C}} \cong \phi_{1,1}^{(9)} \oplus \phi_{1,7}^{(9)} .
$$

The extension is effected by a field of scaling dimension 8 , and the index $\left[\mathcal{C}_{\max }\right.$ : $\left.\mathcal{V} i r_{c(9)}\right]$ coincides with the statistical dimension of $\rho^{\mathcal{C}}$, which we calculate using (V.39) as: $d\left(\rho^{\mathcal{C}}\right)=3+\sqrt{3}$. There is only one local extension of $\mathcal{V}_{i r_{c(9)}}$ with this index, namely the $\left(A_{10}, E_{6}\right)$ extension [KL02, table 3], which is hence identified as a Coset model.

$\mathcal{A}_{\text {max }}$ is a non-trivial local extension of $\mathcal{L} S U(9)_{2}$, and it is completely rational, as $\mathcal{L} S U(9)_{2}$ is included with finite index [Lon01]. From arguments above we know that $\mathcal{A}_{\max } \subseteq \mathcal{C}_{\max } \subset \mathcal{L} E(8)_{2}$ is a normal CTPS (proposition V.14). We want to derive the decomposition of $\rho_{\max }$ from (V.40). It is clear that it has the form of (V.33), where each $\rho_{u}^{\mathcal{A}_{\max }}\left(\rho_{v}^{\mathcal{C}_{\max }}\right)$ appears only once, and for each $\rho_{u}^{\mathcal{A}_{\max }}\left(\rho_{v}^{\mathcal{C}_{\max }}\right)$ the conjugate $\rho_{\bar{u}}^{\mathcal{A}_{\max }}\left(\rho_{\bar{v}}^{\mathcal{C}_{\max }}\right)$ has to appear as well [Reh00]. We know that all the representations occurring in $(\mathrm{V} .40)$ come from restrictions of the $\rho_{u}^{\mathcal{A}_{\max }}, \rho_{v}^{\mathcal{C}_{\max }}$.

Any sector of $\mathcal{V} i r_{c(9)}$ appearing in (V.40) appears only once and they all are self-conjugate. Looking at the restricted fusion rules of conjugate sectors of $\mathcal{C}_{\text {max }}$, equation (V.34), we conclude: if a $\phi_{2 l+1,1}^{(9)}$ appearing in (V.40) is the restriction of a $\rho_{v}^{\mathcal{C}_{\max }}$, then its square has to contain $\rho^{\mathcal{C}}$. But this is not the case, as one may verify using the fusion rules (V.38). This shows that the restrictions of irreducible $\rho_{v}^{\mathcal{C}_{\max }}$ contained in $\rho_{\max }$ are of the form $\bar{\iota}^{\mathcal{C}} \rho_{v}^{\mathcal{C}_{\max }} \iota^{\mathcal{C}} \cong \phi_{2 l+1,1}^{(9)} \oplus \phi_{2 l+1,7}^{(9)}$, with $l$ depending on $v$.

Now we may calculate the statistical dimensions of the irreducible $\rho_{v}^{\mathcal{C}_{\max }}$ contained in $\rho_{\max }$ from (V.40), (V.39) according to [Car02, proposition 3.1]:

$$
d\left(\bar{\iota}^{\mathcal{C}} \rho_{v}^{\mathcal{C}_{\text {max }}} \iota^{\mathcal{C}}\right)=d\left(\rho_{v}^{\mathcal{C}_{\text {max }}}\right)\left[\mathcal{C}_{\text {max }}: \mathcal{V} i r_{c(9)}\right]=d\left(\rho_{v}^{\mathcal{C}_{\text {max }}}\right) d\left(\rho^{\mathcal{C}}\right) .
$$


The statistical dimensions of the representations of $\mathcal{V} i r_{c(9)}$ occurring in (V.40) are given by:

\begin{tabular}{c|ccc}
$l$ & $d_{2 l+1,1}^{(9)}$ & $d_{2 l+1,7}^{(9)}$ & $\left(d_{2 l+1,1}^{(9)}+d_{2 l+1,7}^{(9)}\right) d\left(\rho^{\mathcal{C}}\right)^{-1}$ \\
\hline 0 & 1 & 3.732051 & 1 \\
1 & 2.682507 & 10.011252 & 2.682507 \\
2 & 3.513337 & 13.111953 & 3.513337 \\
3 & 3.228707 & 12.049700 & 3.228707 \\
4 & 1.918986 & 7.161753 & 1.918986
\end{tabular}

The fusion rules of positive-energy representations $\mathcal{L} S U(9)_{2}$ are known [Was98] and, therefore, the corresponding statistical dimensions may be calculated as asymptotic dimensions using formulae in, for example, [KW88]. The dimensions for representations occurring in (V.40) are:

$$
\begin{aligned}
d_{2 \dot{\Lambda}_{0}}=d_{2 \dot{\Lambda}_{3}}=d_{2 \dot{\Lambda}_{6}}=1, \\
d_{\dot{\Lambda}_{1}+\dot{\Lambda}_{8}}=d_{\dot{\Lambda}_{4}+\dot{\Lambda}_{2}}=d_{\dot{\Lambda}_{7}+\dot{\Lambda}_{5}} \approx 2.682507, \\
d_{\dot{\Lambda}_{2}+\dot{\Lambda}_{7}}=d_{\dot{\Lambda}_{5}+\dot{\Lambda}_{1}}=d_{\dot{\Lambda}_{8}+\dot{\Lambda}_{4}} \approx 3.513337, \\
d_{\dot{\Lambda}_{3}+\dot{\Lambda}_{6}}=d_{\dot{\Lambda}_{6}+\dot{\Lambda}_{0}}=d_{\dot{\Lambda}_{0}+\dot{\Lambda}_{3}} \approx 3.228707, \\
d_{\dot{\Lambda}_{4}+\dot{\Lambda}_{5}}=d_{\dot{\Lambda}_{7}+\dot{\Lambda}_{8}}=d_{\dot{\Lambda}_{1}+\dot{\Lambda}_{2}} \approx 1.918986 .
\end{aligned}
$$

Below $\sqrt{6}$ the numbers which can occur as a statistical dimension form a discrete set and the only value below $\sqrt{2}$ is 1 [Jon83, Reh95]. This allows to write actual equalities in the first line, which implies: $\left[\mathcal{A}_{\text {max }}: \mathcal{L} S U(9)_{2}\right]=d\left(\rho^{\mathcal{A}}\right)=3$. Through the corresponding version of equation (V.45) the statistical dimensions of candidate $\rho_{u}^{\mathcal{A}_{\max }}$ given by thinkable decompositions of $\rho_{\max }$ may be calculated. These have to coincide with the dimension of the $\rho_{v}^{\mathcal{C}_{\max }}$ they are coupled to [Reh00, theorem 3.6]. Comparing (V.46) and (V.47), this requirement admits the decomposition of $\rho_{\max }$ only to be given by (V.41), (V.42), (V.43).

The representations $\rho_{l}^{\mathcal{A}_{\max }}, \rho_{l}^{\mathcal{C}_{\max }}$ are all self-conjugate, because the $\phi_{r, s}^{(m)}$ all are and the coupling matrix induces an isomorphism of fusion rules [Reh00].

Remark: According to [KW88, 4.1.b], the $\left(A_{10}, E_{6}\right)$ extension of $\mathcal{V} i r_{c(9)}$ may as well be viewed as the maximal COSET model associated with the chiral subnet $\mathcal{L} E(8)_{3} \subset \mathcal{L} E(8)_{2} \otimes \mathcal{L} E(8)_{1}$.

Concerning the forth exceptional local extension of a $\mathcal{V} i r_{c<1}$ model, namely the $\left(A_{28}, E_{8}\right)$ extension of $\mathcal{V} i r_{c(27)}$, the classification of current subalgebras with Coset stress-energy tensor having $c<1$ [BG87] and the classification of conformal inclusions of current algebras [AGO87, BB87, SW86] suggests that this is probably not a COSET model associated with a current subalgebra. Nevertheless, this chiral conformal theory is known to exist by an abstract construction relying on DHR data of $\mathcal{V} i r_{c(27)}$ [KL02] and thus appears to be a genuine achievement of the conceptual approach of local quantum physics. 


\subsection{A normal CTPS in $\mathcal{L} E(8)_{1}$}

As an example for the structure discussed by MüGER [Müg02] we discuss a COSET pair in $\mathcal{L} E(8)_{1}$. This we will show first: Up to unitary equivalence the vacuum representation is the only locally normal representation of $\mathcal{L} E(8)_{1}$. If we look only at the representations that stem from integrating a unitarisable highestweight representation of the corresponding current algebra, this is clear because the WEYL-alcove condition only admits one highest weight, namely the vacuum. But we need a statement on the chiral model $\mathcal{L} E(8)_{1}$, which we obtain applying well-known statements on completely rational models and our current knowledge of the models $\mathcal{L} S U(n)_{k}$.

Proposition V.16: $\mathcal{L} E(8)_{1}$ is completely rational and its only sector is the vacuum sector.

Proof: Complete rationality of $\mathcal{L} E(8)_{1}$ holds, because $\mathcal{L} S U(9)_{1} \subset \mathcal{L} E(8)_{1}$ is a conformal inclusion. Indeed, the branching of this chiral subnet reads [KNS88]:

$$
L\left(\Lambda_{0}\right) \Upsilon \cong L\left(\dot{\Lambda}_{0}\right)+L\left(\dot{\Lambda}_{3}\right)+L\left(\dot{\Lambda}_{6}\right) .
$$

By the symbol $\uparrow$ we indicate that the formula gives the decomposition of $L\left(\Lambda_{0}\right)$ as a representation of $\mathcal{L} S U(9)_{1}$. Again the notation is standard for representations of affine KAC-Moody algebras (see [KW88] for a summary): $L\left(\Lambda_{0}\right)$ is the vacuum representation of $\mathcal{L} E(8)_{1}, L\left(\dot{\Lambda}_{0}\right)$ is the vacuum representation and $L\left(\dot{\Lambda}_{3,6}\right)$ are inequivalent highest-weight representations of $\mathcal{L} S U(9)_{1}$.

To prove that $\mathcal{L} E(8)_{1}$ has trivial superselection structure, we calculate its $\mu$-index which turns out to be 1 ; this is a necessary and sufficient condition for trivial superselection structure for completely rational chiral nets [KLM01, corollary 32]. The identity $\mu_{\mathcal{L E}(8)_{1}}=1$ appears implicitly in [Sta95] as well, but we prefer giving a self-contained proof. We know that the $\mu$-index of $\mathcal{L} S U(9)_{1}$ is 9 [Xu00b, section 4.4] and since there are 9 locally normal representations of $\mathcal{L} S U(9)_{1}$ (cf remarks at the end of section III.2) each of them has statistical dimension 1. Hence, the index of the conformal inclusion is determined to be: $\left[\mathcal{L} E(8)_{1}: \mathcal{L} S U(9)_{1}\right]=3$.

The $\mu$-index of $\mathcal{L} E(8)_{1}$ may be calculated using the conformal inclusion above [KLM01, proposition 24]:

$$
\mu\left(\mathcal{L S U}(9)_{1}\right)=\left[\mathcal{L} E(8)_{1}: \mathcal{L} S U(9)_{1}\right]^{2} \mu\left(\mathcal{L} E(8)_{1}\right) .
$$

This yields $\mu\left(\mathcal{L} E(8)_{1}\right)=1$. 
Now we present the example itself, the Coset pair $\mathcal{L} S U(2)_{16} \subset \mathcal{L} S U(3)_{6} \subset$ $\mathcal{L} E(8)_{1}$. It has the branching [KNS88]:

$$
\begin{aligned}
& L\left(\Lambda_{0}\right) \uparrow \cong {\left[L\left(16 \dot{\Lambda}_{0}\right) \oplus L\left(16 \dot{\Lambda}_{1}\right)\right] \otimes\left[L\left(6 \ddot{\Lambda}_{0}\right) \oplus L\left(6 \ddot{\Lambda}_{1}\right) \oplus L\left(6 \ddot{\Lambda}_{2}\right)\right] } \\
& \oplus\left[L\left(14 \dot{\Lambda}_{0}+2 \dot{\Lambda}_{1}\right) \oplus L\left(2 \dot{\Lambda}_{0}+14 \dot{\Lambda}_{1}\right) \oplus 2 L\left(8 \dot{\Lambda}_{0}+8 \dot{\Lambda}_{1}\right)\right] \\
& \quad \otimes L\left(2 \ddot{\Lambda}_{0}+2 \ddot{\Lambda}_{1}+2 \ddot{\Lambda}_{2}\right) \\
& \oplus\left[L\left(10 \dot{\Lambda}_{0}+6 \dot{\Lambda}_{1}\right) \oplus L\left(6 \dot{\Lambda}_{0}+10 \dot{\Lambda}_{1}\right)\right] \\
& \quad \otimes\left[L\left(4 \ddot{\Lambda}_{0}+\ddot{\Lambda}_{1}+\ddot{\Lambda}_{2}\right) \oplus L\left(\ddot{\Lambda}_{0}+4 \ddot{\Lambda}_{1}+\ddot{\Lambda}_{2}\right) \oplus L\left(\ddot{\Lambda}_{0}+\ddot{\Lambda}_{1}+4 \ddot{\Lambda}_{2}\right)\right] \\
& \oplus\left[L\left(12 \dot{\Lambda}_{0}+4 \dot{\Lambda}_{1}\right) \oplus L\left(4 \dot{\Lambda}_{0}+12 \dot{\Lambda}_{1}\right)\right] \\
& \otimes\left[L\left(3 \ddot{\Lambda}_{0}+3 \ddot{\Lambda}_{1}\right) \oplus L\left(3 \ddot{\Lambda}_{0}+3 \ddot{\Lambda}_{2}\right) \oplus L\left(3 \ddot{\Lambda}_{1}+3 \ddot{\Lambda}_{2}\right)\right] . \quad \text { (V.50) }
\end{aligned}
$$

By the symbol $\uparrow$ we indicate that the formula gives the decomposition of $L\left(\Lambda_{0}\right)$ as a representation of $\mathcal{L} S U(2)_{16} \otimes \mathcal{L} S U(3)_{6}$.

Again, the notation is the usual one for highest-weight representations of affine KAC-Moody algebras (summary eg in [KW88]): $\Lambda_{0}$ stands for the vacuum representation at level 1 of the affine KAC-MOODY algebra with horizontal subalgebra $E(8), \dot{\Lambda}_{i}$ for the $i$ th fundamental weight of the affine KAC-Moody algebra with horizontal subalgebra isomorphic to the LIE algebra of $S U(2)$ and $\ddot{\Lambda}_{i}$ for the $i$ th fundamental weight of the affine KAC-MOODY algebra with horizontal subalgebra isomorphic to the LIE algebra of $S U(3)$. If one is interested in the highest weight of the modules of $S U(2)$ or $S U(3)$ formed by the vectors of lowest energy, one simply ignores the multiple of $\dot{\Lambda}_{0}$ and $\ddot{\Lambda}_{0}$ and takes $\dot{\Lambda}_{i}, \ddot{\Lambda}_{i}, i \neq 0$, to stand for the corresponding fundamental weights of $S U(2), S U(3)$, respectively.

The highest weight vectors of representations of $\mathcal{L} S U(2)_{16}$ occurring in (V.50) have the following conformal energies:

$$
\begin{aligned}
h\left(16 \dot{\Lambda}_{0}\right)=0, & h\left(14 \dot{\Lambda}_{0}+2 \dot{\Lambda}_{1}\right)=\frac{1}{9}, \quad h\left(2 \dot{\Lambda}_{0}+14 \dot{\Lambda}_{1}\right)=\frac{28}{9} \\
h\left(16 \dot{\Lambda}_{1}\right)=4, & h\left(8 \dot{\Lambda}_{0}+8 \dot{\Lambda}_{1}\right)=\frac{10}{9}, \\
h\left(10 \dot{\Lambda}_{0}+6 \dot{\Lambda}_{1}\right) & =\frac{2}{3}, \quad h\left(6 \dot{\Lambda}_{0}+10 \dot{\Lambda}_{1}\right)=\frac{5}{3} \\
h\left(12 \dot{\Lambda}_{0}+4 \dot{\Lambda}_{1}\right) & =\frac{1}{3}, \quad h\left(4 \dot{\Lambda}_{0}+12 \dot{\Lambda}_{1}\right)=\frac{7}{3} .
\end{aligned}
$$

Since most of these lowest energy eigenvalues are not half-integers, the innerimplementing representation $U^{\mathcal{L S U}(2)_{16}}$ can not have sharp geometrical action (proposition V.12). 
We introduce some shorthand notation by defining, up to unitary equivalence, representations of the respective chiral net localised in some $I \Subset \mathrm{S}^{1}$ :

$$
\begin{aligned}
\alpha_{(0)}: \cong L\left(16 \dot{\Lambda}_{0}\right) \oplus L\left(16 \dot{\Lambda}_{1}\right) \\
\alpha_{(1)}: \cong L\left(14 \dot{\Lambda}_{0}+2 \dot{\Lambda}_{1}\right) \oplus L\left(2 \dot{\Lambda}_{0}+14 \dot{\Lambda}_{1}\right) \\
\alpha_{(2)}: \cong L\left(8 \dot{\Lambda}_{0}+8 \dot{\Lambda}_{1}\right) \\
\alpha_{(3)}: \cong L\left(8 \dot{\Lambda}_{0}+8 \dot{\Lambda}_{1}\right) \\
\alpha_{(4)}: \cong L\left(10 \dot{\Lambda}_{0}+6 \dot{\Lambda}_{1}\right) \oplus L\left(6 \dot{\Lambda}_{0}+10 \dot{\Lambda}_{1}\right) \\
\alpha_{(5)}: \cong L\left(12 \dot{\Lambda}_{0}+4 \dot{\Lambda}_{1}\right) \oplus L\left(4 \dot{\Lambda}_{0}+12 \dot{\Lambda}_{1}\right) . \\
\zeta_{(0)}: \cong L\left(6 \ddot{\Lambda}_{0}\right) \oplus L\left(6 \ddot{\Lambda}_{1}\right) \oplus L\left(6 \ddot{\Lambda}_{2}\right) \\
\zeta_{(1)}: \cong L\left(2 \ddot{\Lambda}_{0}+2 \ddot{\Lambda}_{1}+2 \ddot{\Lambda}_{2}\right) \\
\zeta_{(2)}: \cong L\left(2 \ddot{\Lambda}_{0}+2 \ddot{\Lambda}_{1}+2 \ddot{\Lambda}_{2}\right) \\
\zeta_{(3)}: \cong L\left(2 \ddot{\Lambda}_{0}+2 \ddot{\Lambda}_{1}+2 \ddot{\Lambda}_{2}\right) \\
\zeta_{(4)}: \cong L\left(4 \ddot{\Lambda}_{0}+\ddot{\Lambda}_{1}+\ddot{\Lambda}_{2}\right) \oplus L\left(\ddot{\Lambda}_{0}+4 \ddot{\Lambda}_{1}+\ddot{\Lambda}_{2}\right) \oplus L\left(\ddot{\Lambda}_{0}+\ddot{\Lambda}_{1}+4 \ddot{\Lambda}_{2}\right) \\
\zeta_{(5)}: \cong L\left(3 \ddot{\Lambda}_{0}+3 \ddot{\Lambda}_{1}\right) \oplus L\left(3 \ddot{\Lambda}_{0}+3 \ddot{\Lambda}_{2}\right) \oplus L\left(3 \ddot{\Lambda}_{1}+3 \ddot{\Lambda}_{2}\right) .
\end{aligned}
$$

All the models involved are completely rational and, therefore, we know that the Coset pair $\mathcal{A}_{\max } \subset \mathcal{C}_{\max } \subset \mathcal{L} E(8)_{1}$ is a normal CTPS. It is straightforward to determine the decomposition of $\rho_{\max }$, the localised, unitarily equivalent representation:

Proposition V.17: The branching for the Coset pair $\mathcal{A}_{\max }\left(\mathcal{C}_{\max } \subset \mathcal{L} E(8)_{1}\right.$ associated with $\mathcal{L} S U(2)_{16} \subset \mathcal{L} S U(3)_{6} \subset \mathcal{L} E(8)_{1}$ reads:

$$
\rho_{\max } \cong \bigoplus_{l=0}^{5} \rho_{l}^{\mathcal{A}_{\max }} \otimes \rho_{l}^{\mathcal{C}_{\text {max }}}
$$

The branchings of the restricted representations read referring to (V.51), (V.52):

$$
\bar{\iota}^{\mathcal{A}} \rho_{l}^{\mathcal{A}_{\max }} \iota^{\mathcal{A}} \cong \alpha_{(l)}, \quad \bar{\iota}^{\mathcal{C}} \rho_{l}^{\mathcal{C}_{\max }} \iota^{\mathcal{C}} \cong \zeta_{(l)}
$$

Proof: The interpretation of the first line in (V.50) is clear from proposition IV.8. The argument from the proof of proposition V.15, using the fusion rules (V.38) only fixes the problem for $l=4,5$ and for $l=1,2,3$ we need to rely entirely on calculating and matching dimensions which turns out to be sufficient to determine the decomposition completely.

We know that the statistical dimensions of irreducible representations of $\mathcal{A}_{\text {max }}$ and $\mathcal{C}_{\text {max }}$, which are coupled in $\rho_{\max }$, have to coincide [Reh00, theorem 3.6]. The 
statistical dimensions of candidate $\rho_{u}^{\mathcal{A}_{\max }}, \rho_{v}^{\mathcal{C}_{\text {max }}}$ contained in thinkable decompositions of $\rho_{\max }$ may be calculated as follows: After having identified $\rho^{\mathcal{A}}, \rho^{\mathcal{C}}$ in (V.50) (proposition IV.8) we immediately get:

$$
\left[\mathcal{A}_{\text {max }}: \mathcal{L} S U(2)_{16}\right]=d\left(\rho^{\mathcal{A}}\right)=2, \quad\left[\mathcal{C}_{\max }: \mathcal{L} S U(3)_{6}\right]=d\left(\rho^{\mathcal{C}}\right)=3 .
$$

For the statistical dimension of representations $\rho_{u}^{\mathcal{A}_{\max }}, \rho_{v}^{\mathcal{C}_{\max }}$ of $\mathcal{A}_{\max }, \mathcal{C}_{\max }$ we have the following (eg [Car02, proposition 3.1]):

$$
\begin{aligned}
d\left(\bar{\iota}^{\mathcal{A}} \rho_{u}^{\mathcal{A}_{\max }} \iota^{\mathcal{A}}\right) & =d\left(\rho_{u}^{\mathcal{A}_{\max }}\right)\left[\mathcal{A}_{\max }: \mathcal{L} S U(2)_{16}\right] \\
d\left(\bar{\iota}^{\mathcal{C}} \rho_{v}^{\mathcal{C}_{\max }} \iota^{\mathcal{C}}\right) & =d\left(\rho_{v}^{\mathcal{C}_{\max }}\right)\left[\mathcal{C}_{\max }: \mathcal{L} S U(3)_{6}\right]
\end{aligned}
$$

Now the statistical dimensions of the irreducible representations of $\mathcal{L} S U(2)_{16}$, $\mathcal{L} S U(3)_{6}$ involved in ( $\left.\mathrm{V} .50\right)$ can be calculated as asymptotic dimensions (these coincide because the respective fusion algebras are identical [Was98]) using formulae in, eg, [KW88]. The results are 4 :

$$
\begin{aligned}
& d_{16 \dot{\Lambda}_{0}}=d_{16 \dot{\Lambda}_{1}}=1, \\
& d_{14 \dot{\Lambda}_{0}+2 \dot{\Lambda}_{1}}=d_{2 \dot{\Lambda}_{0}+14 \dot{\Lambda}_{1}} \approx 2.879385, \\
& d_{8 \dot{\Lambda}_{0}+8 \dot{\Lambda}_{1}} \approx 5.758770 \\
& d_{1 \dot{0} \Lambda_{0}+6 \dot{\Lambda}_{1}}=d_{\dot{6} \Lambda_{0}+10 \dot{\Lambda}_{1}} \approx 5.411474, \\
& d_{12 \dot{\Lambda}_{0}+4 \dot{\Lambda}_{1}}=d_{4 \dot{\Lambda}_{0}+12 \dot{\Lambda}_{1}} \approx 4.411474 \text {. } \\
& d_{6 \ddot{\Lambda}_{0}}=d_{6 \ddot{\Lambda}_{1}}=d_{6 \ddot{\Lambda}_{2}}=1,
\end{aligned}
$$

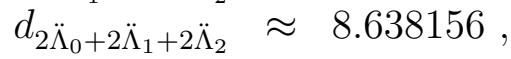

$$
\begin{aligned}
& d_{4 \ddot{\Lambda}_{0}+\ddot{\Lambda}_{1}+\ddot{\Lambda}_{2}}=d_{\ddot{\Lambda}_{0}+4 \ddot{\Lambda}_{1}+\ddot{\Lambda}_{2}}=d_{\ddot{\Lambda}_{0}+\ddot{\Lambda}_{1}+4 \ddot{\Lambda}_{2}} \approx 5.411474, \\
& d_{3 \ddot{\Lambda}_{0}+3 \ddot{\Lambda}_{1}}=d_{3 \ddot{\Lambda}_{0}+3 \ddot{\Lambda}_{2}}=d_{3 \ddot{\Lambda}_{1}+3 \ddot{\Lambda}_{2}} \approx 4.411474 \text {. }
\end{aligned}
$$

If one compares the resulting statistical dimensions of candidate $\rho_{u}^{\mathcal{A}_{\text {max }}}, \rho_{v}^{\mathcal{C}_{\text {max }}}$ coupled in $\rho_{\max }$ according to the equations (V.54), (V.55), (V.56), (V.57) one finds that this only admits a decomposition of $\rho_{\max }$ of the form claimed above.

Remarks: Simply by looking at the branching (V.50) one probably conjectured a decomposition of $\rho_{\max }$ into 4 tensor products of irreducibles rather than six. MÜGER's results [Müg02] show, that the connection between sectors coupled in the branching (V.53) induces an isomorphism of the DHR subcategories generated by the sectors of $\mathcal{A}_{\max }$ and $\mathcal{C}_{\max }$ which are contained in $\rho_{\max }$.

\footnotetext{
${ }^{4}$ Below $\sqrt{6}$ numerical calculations of sufficient accuracy allow complete identification of the dimensions, since the allowed dimensions of localised representations below $\sqrt{6}$ are known to form a discrete set [Jon83, Reh95]; the only value below $\sqrt{2}$ is 1 .
} 


\section{Chapter VI}

\section{The conformally covariant derivatives of the $U(1)$ current}

Much of the present understanding of quantum field theories was achieved by methods related to internal and spacetime symmetries. There are reasons to be interested in objects connected with symmetries which are of a local nature: the lemmas IV.2, IV.12, for example, were proved assuming the presence of a stress-energy tensor. These two lemmas are the foundation of the analysis on the isotony problem, they led to the concept of chiral holography and should hold in quite general circumstances. In this chapter we establish limitations of the concept of a stress-energy tensor and, hence, add reasons for being interested in densities generating specific spacetime symmetries in a more general sense.

Within the classical framework the relation between local objects and continuous symmetries of a Lagrangean field theory is canonical by NoETHER's theorem: to each such symmetry we have an explicitly known conserved current, whose integrals over space, the charges, generate the corresponding symmetry transformation. In quantum field theory the situation is less satisfactory. If one quantises a classical Lagrangean field theory, it may happen that some symmetries do not survive at all because of renormalisation effects. Moreover, there is no a priori knowledge of densities connected with continuous symmetries of a general quantum field theory, although it is possible to characterise such fields

abstractly, of course. The nature of conserved currents connected to symmetries at the quantum level (and of their charges in particular) is hard to clarify in general.

These problems are more accessible for the global conformal spacetime symmetry in $1+1$ dimensions. Here we have an abundance of models for which explicit constructions of a conserved WIGHTMAN quantum field are known, which serves as a density for the conformal symmetry. When smeared out with suitable testfunctions, this field actually generates the conformal symmetry in the sense of integrable LIE algebra representations. Its interpretation as a stress-energy tensor is in direct analogy with the classical object. Depending on weak assumptions 
LÜSCHER AND MACK found that stress-energy tensors of conformally covariant quantum field theories in $1+1$ dimensions always yield local formulations of the VIRASORO algebra [Mac88, LM76, FST89]. In chapter III we discussed current algebras as examples of this structure.

We prove: No such stress-energy tensor exists in a class of completely wellbehaved conformal theories in $1+1$ dimensions, the conformally covariant derivatives of the $U(1)$ current. These are constructed as fields on Minkowski space and possess conformally covariant extensions on their own Fock space, but they do not transform covariantly with respect to the transformations implementing global conformal symmetry of the $U(1)$ current.

YNGVASON [Yng94] studied the conformally covariant derivatives as part of a broader class of derivatives of the $U(1)$ current and established, among other things, that they do not fulfill HaAg duality on Minkowski space ${ }^{1}$. Guido, LONGO AND WIESBROCK [GLW98] studied locally normal representations of these models and found representations of the first derivative, which do not allow an implementation of global conformal symmetry. In a closing side-remark they noted that this contradicts, by unpublished results of D'ANTONI AND FredenHAGEN, presence of diffeomorphism symmetry in these models.

This symmetry is not manifest in the commutation relations of local fields in this model, but as these only serve as "field coordinates" for the local quantum theory they generate, one has to have a closer look for a complete proof, see section A,5. At this point we are interested in a direct argument that excludes presence of a stress-energy tensor for the derivative models.

Most of this chapter is also available as [Kös03a].

\section{The chiral nets generated by the $\Phi^{(n)}$}

The $U(1)$ current in $1+1$ dimensions decomposes into two independent chiral components, the chiral currents, and we shall discuss one of these only (cf chapter III). The derivatives of the chiral current $j$ are given as fields on the light-ray by $\Phi^{(n)}(x):=\partial_{x}^{n} j(x)$, where we used $\partial_{x}:=d / d x$. These fields are covariant with scaling dimension $n+1$ when acted upon by the implementation of the stabiliser group of $\infty$ for the $U(1)$-current theory. By looking at their Wightman functions one recognises that the $\Phi^{(n)}$ possess conformally covariant extensions, if restricted to their own FOCK space; the corresponding unitary representation of $\operatorname{PSL}(2, \mathbb{R})$ implementing global conformal symmetry leaves invariant the vacuum and fulfills the spectrum condition. Each of these extensions transforms covariantly with respect to a different representation of the global conformal group and lives on a different Fock space. From now on, we look at the fields as operators in their

\footnotetext{
${ }^{1}$ Essential duality, which is another name for HAAG duality on the conformal covering of Minkowski space, is a consequence of conformal symmetry [BGL93].
} 
cyclic subrepresentation equipped with their own representation of the global conformal group and we use the symbol $\Phi^{(n)}$ in this sense.

By construction, the derivative fields $\Phi^{(n)}$ obey the following commutation relation as fields on the light-ray:

$$
\left[\Phi^{(n)}(x), \Phi^{(n)}(y)\right]=\frac{i}{2 \pi}(-)^{n} \delta^{(2 n+1)}(x-y) \mathbb{1} .
$$

We want to calculate the corresponding commutation relations for the modes of the conformally extended fields on the compactified light-ray, $\mathrm{S}^{1}$. These fields will be denoted $\widetilde{\Phi}^{(n)}$. The calculation is done best in terms of smeared fields, for details see section III,2. The testfunctions of fields on $\mathrm{S}^{1}$ and their images living on the light-ray are connected by a transformation $f \mapsto \widehat{f}$ depending on the scaling dimension of the respective field; its definition is induced by $\widetilde{\Phi}^{(n)}(f) \equiv \Phi^{(n)}(\widehat{f})$.

Proposition VI.1: The modes $\Phi_{m}^{(n)}:=\widetilde{\Phi}^{(n)}\left(\left[z^{n+m}\right]\right)$ have the following commutation relations:

$$
\left[\Phi_{m}^{(n)}, \Phi_{m^{\prime}}^{(n)}\right]=\delta_{m,-m^{\prime}} \Pi^{(n)}(m),
$$

if we set $\Pi^{(n)}(m):=\prod_{k=0}^{2 n}(m-n+k)$.

Remark: These relations imply that the modes $\Phi_{m}^{(n)},|m| \leq n$, are central, which in turn means that all $L_{0}$-eigenspaces for eigenvalues $1, \ldots, n$ are null.

Proof: We use the shorthand notations $\zeta:=(1+z), d / d z=\partial_{\zeta}$ and arrive at:

$$
\begin{aligned}
& {\left[\widetilde{\Phi}^{(n)}(f), \widetilde{\Phi}^{(n)}(g)\right] \equiv\left[\Phi^{(n)}(\widehat{f}), \Phi^{(n)}(\widehat{g})\right] } \\
= & (-)^{2 n+1} \oint \frac{d z}{2 \pi i} f(z) \zeta^{-2(n+1)}\left(\zeta^{2} \frac{d}{d \zeta}\right)^{2 n+1} \zeta^{-2 n} g(z) \\
= & \oint \frac{d z}{2 \pi i} g(z)\left(\frac{d}{d z}\right)^{2 n+1} f(z) .
\end{aligned}
$$

The identity of the two integration kernels as distributions may be proved inductively. Applying the induction assumption we see that we have to prove: $\zeta^{-2 n} \partial_{\zeta} \zeta^{2} \partial_{\zeta} \zeta^{2 n} \partial_{\zeta}^{2 n-1} \zeta^{-2}=\partial_{\zeta}^{2 n+1}$. One may verify this identity for $n=1$ explicitly. Then one proves by induction on $n$ :

$$
\begin{aligned}
& \zeta^{-2(n+1)} \partial_{\zeta} \zeta^{2} \partial_{\zeta} \zeta^{2(n+1)} \partial_{\zeta}^{2 n+1} \zeta^{-2} \\
= & \zeta^{-2} \partial_{\zeta}^{2 n+1}\left(\zeta^{2} \partial_{\zeta}^{2}-2(2 n+1) \zeta^{2} \partial_{\zeta} \zeta^{-1}+(2 n+1)(2 n)\right)=\partial_{\zeta}^{2 n+3} .
\end{aligned}
$$

As we can see by looking at their canonical commutation relations, the derivative fields may be treated as local quantum theories of bounded operators in terms of WEYL operators and their relations (cf [GLW98]). We take another approach 
which was introduced by BuchHolz And Schulz-Mirbach [BSM90] for the nets of the stress-energy tensor and the $U(1)$ current: By establishing linear energy bounds referring to the conformal Hamiltonian $L_{0}$ the HAAG-KAstLER axioms follow from WightmaN's set of axioms. In particular the fields are essentially self-adjoint on the WIGHTMAN domain, their bounded functions fulfill locality and the local algebras generate a dense subspace from the vacuum. The local algebras are generated by unitaries $W(f):=\exp \left(i \widetilde{\Phi}^{(n)}(f)^{-}\right), \widetilde{\Phi}^{(n)}(f)^{-}$selfadjoint and $\operatorname{supp}(f) \Subset \mathrm{S}^{1}$. The $W(f)$ are concrete representations of the WEYL operators.

Proposition VI.2: The following defines the local algebras of the chiral net generated by the $\Phi^{(n)}$ fields:

$$
\mathcal{A}_{\Phi^{(n)}}(I):=\left\{\widetilde{\Phi}^{(n)}(f)^{-}, \operatorname{supp}(f) \subset I, f=\widetilde{\widetilde{\widehat{f}}}\right\}^{\prime \prime}, I \Subset \mathrm{S}^{1} .
$$

Proof: The proof follows the lines indicated in [BSM90]. If $\psi_{N}$ denotes an arbitrary eigenvector of $L_{0}$ with energy $N$ and norm 1 , then $\Phi_{m}^{(n)} \psi_{N}, m>0$, is a multiple of a unit vector of energy $N-m$, which we will call $\psi_{N-m}$. Making use of a general estimate for positive, linear functionals $\eta\left[\right.$ Buc90]: $|\eta(Q)|^{2} \leq \eta\left(Q^{*} Q\right)$, we are led to the following estimate:

$$
\left\|\Phi_{m}^{(n)} \psi_{N}\right\|^{4} \leqslant\left\|\Phi_{m}^{(n)} \psi_{N}\right\|^{2}\left\|\Phi_{m}^{(n)} \psi_{N-m}\right\|^{2}+\Pi^{(n)}(m)\left\|\Phi_{m}^{(n)} \psi_{N}\right\|^{2} .
$$

For $m \neq 0$ we set $\Pi^{\prime(n)}(m):=\frac{1}{m} \Pi^{(n)}(m)$ and we prove inductively using the spectrum condition: $\left\|\Phi_{m}^{(n)} \Psi_{N}\right\|^{2} \leq N \Pi^{\prime(n)}(m), m \geq 1$.

For the generating modes we have:

$$
\left\|\Phi_{-m}^{(n)} \Psi_{N}\right\|^{2}=\left\|\Phi_{m}^{(n)} \Psi_{N}\right\|^{2}+\Pi^{(n)}(m) \leq(N+m) \Pi^{\prime(n)}(m) .
$$

The zeroth mode is central in the theory and is, therefore, a multiple $q$ of the identity. So we have: $\left\|\Phi_{0}^{(n)} \Psi_{N}\right\|^{2}=q^{2}, q \in \mathbb{R}$. For general $\Phi^{(n)}(f), f \in C^{\infty}\left(\mathrm{S}^{1}\right)$, and a vector $\Psi$ from the WIGHTMAN domain we have the following estimate:

$$
\left\|\Phi^{(n)}(f) \Psi\right\| \leqslant\left\|\left(L_{0}+\mathbb{1}\right) \Psi\right\| \sum_{m \in \mathbb{Z}}\left|f_{m}\right|\left(|m|+\Pi^{\prime(n)}(m)+|q|+1\right) .
$$

This is the linear energy bound from which the HAAG-KASTLER axioms follow as discussed in the proof of theorem III.1.

GABBIANI AND FröHLICH [GF93] gave a formulation of the nuclearity condition adequate for chiral nets, following [BDF87]. We start with a review on this formulation and relate it to properties of $L_{0}$ in typical chiral models. First, we define maps $\Theta_{\beta}, \beta>0$, from the local algebras of a chiral conformal model $\mathcal{B}$ in its vacuum representation into its vacuum HILBERT space $\mathscr{H}$ by:

$$
\Theta_{\beta}: \mathcal{B}(I) \rightarrow \mathscr{H}, B \mapsto e^{-\beta L_{0}} B \Omega, \beta>0
$$


The functions $\Theta_{\beta}$ are required to be nuclear. This means that for every $\Theta_{\beta}$ there exist a sequence of vectors $\phi_{\iota} \in \mathscr{H}$ and a sequence of linear functionals $\varphi_{\iota} \in \mathcal{B}(I)^{*}$ such that:

$$
\Theta_{\beta}(.)=\sum_{\iota} \varphi_{\iota}(.) \phi_{\iota}, \quad \sum_{\iota}\left\|\varphi_{\iota}\right\|\left\|\phi_{\iota}\right\| \leqslant \infty .
$$

Furthermore, defining the trace-norm of $\Theta_{\beta}$ by: $\left\|\Theta_{\beta}\right\|_{1}:=\inf \sum_{\iota}\left\|\varphi_{\iota}\right\|\left\|\phi_{\iota}\right\|$, where the infimum is to be taken over all sequences $\left(\phi_{\iota}\right)_{\iota},\left(\varphi_{\iota}\right)_{\iota}$ complying with the conditions above, there shall be an asymptotic bound for $\beta \searrow 0$ :

$$
\left\|\Theta_{\beta}\right\|_{1} \leqslant e^{\left(\frac{\beta_{0}}{\beta}\right)^{n}}, \beta_{0}, n>0 .
$$

$\beta_{0}, n$ are constants, depending on $I \Subset \mathrm{S}^{1}$, possibly.

In typical chiral models, $L_{0}$ has a total set of eigenvectors, $e^{-\beta L_{0}}$ is trace-class for $\beta>0$, and its trace has good asymptotic properties for $\beta \backslash 0$ (eg [Kac90, (13.13.14)] and below). With these structures it is straightforward to establish the nuclearity condition (VI.6), (VI.7). If $\left\{\phi_{N, i}\right\}_{N, i}$ is an orthonormal basis of eigenvectors of $L_{0}$-eigenvalue $N$ labelled by an additional multiplicity index $i$, then the expansion in equation (VI.6) reads:

$$
\Theta_{\beta}(.)=\sum_{N, i}\left\langle e^{-\beta L_{0}} \phi_{N, i}, \Omega\right\rangle\left|\phi_{N, i}\right\rangle \equiv \sum_{N, i} \varphi_{N, i}(.)\left|\phi_{N, i}\right\rangle .
$$

and the following estimate holds: $\sum_{N, i}\left\|\varphi_{N, i}\right\|\left\|\phi_{N, i}\right\| \leq \operatorname{Tr}\left(e^{-2 \beta L_{0}}\right)<\infty$. This inequality holds for $\left\|\Theta_{\beta}\right\|_{1}$ as well and the asymptotic properties of $\operatorname{Tr}\left(e^{-2 \beta L_{0}}\right)$ yield a bound of the form (VI.7).

Now we turn to the nuclearity condition for the conformally covariant derivatives of the $U(1)$ current. Each of their Fock spaces is the quotient of a Verma module modulo the space of null vectors. The VERMA module has at each energy level $N$ a basis given by vectors $\Phi_{-m_{1}}^{(n)} \ldots \Phi_{-m_{k}}^{(n)} \Omega, \sum_{i} m_{i}=N, 0<m_{1} \leq \ldots \leq m_{k}$. Since null vectors reduce the multiplicity of $L_{0}$ eigenvalues, the trace of $e^{-\beta L_{0}}$ in the vacuum representation of the derivative models is dominated by the corresponding $L_{0}$-character for the $U(1)$ current, whose Verma module does not contain any null vectors. The following discussion applies for the same reason to all theories defined by a stress-energy tensor, and to the $U(1)$-current algebra itself, of course.

The trace of $e^{-\beta L_{0}}$ in the vacuum representation of the $U(1)$ current coincides with the combinatorial partition function $p\left(e^{-\beta}\right)$, which is directly connected to Dedekind's $\eta$-function:

$$
p\left(e^{-\beta}\right)^{-1}=\prod_{m \geq 1}\left(1-e^{-\beta m}\right)=e^{-\frac{\beta}{24}} \eta(i \beta /(2 \pi)) .
$$

For the nuclearity condition we have to check the asymptotic behaviour for $\beta \backslash 0$. This behaviour is determined by the transformation law of $\eta$ for $\tau \mapsto 1 / \tau$. This 
reads [Sch74, III.§3]: $\sqrt{\beta / 2 \pi} \eta(i \beta /(2 \pi))=\eta(i 2 \pi / \beta)$. We have with $\beta_{0}>-1+$ $\pi^{2} / 6$ and $n=1$ :

$$
\lim _{\beta \backslash 0} p\left(e^{-\beta}\right) e^{-\left(\frac{\beta_{0}}{\beta}\right)^{n}}=0
$$

This estimate is a special form of a nuclearity condition and ensures the split property for all models under consideration by arguments as given in [GF93, lemma 2.12].

\section{No stress-energy tensor in $\Phi^{(n)}$-models}

We seek for a stress-energy tensor in the theories defined by conformally covariant derivatives of degree $n$ of the $U(1)$ current in $1+1$ dimensions. We assume the stress-energy tensor to deserve its name and therefore it should be a local, covariant, conserved, symmetric, traceless quantum field $\Theta$ of scaling dimension 2 , which is relatively local to the $\Phi^{(n)}$ under consideration and a density for its infinitesimal conformal transformations. Because all models involved factorise into chiral components, we shall discuss the situation on the compactified light-ray, ie the fields live on $\mathrm{S}^{1}$.

According to the analysis of LÜSCHER AND MACK the commutation relations

of $\widetilde{\Theta}$ have a very specific form [LM76, Mac88] [FST89, theorem 3.1]. $\widetilde{\Theta}$ is a LIE field with an extension proportional to $c$, the central charge of $\widetilde{\Theta}$ :

$$
\frac{c}{12} \oint \frac{d z}{2 \pi i} f^{\prime \prime \prime}(z) g(z)=[\widetilde{\Theta}(f), \widetilde{\Theta}(g)]-\widetilde{\Theta}\left(f^{\prime} g-f g^{\prime}\right)
$$

$c / 2$ is the normalisation constant of the two point function of $\widetilde{\Theta}$, hence we have $c \in \mathbb{R}_{+}$, and, by the REeH-Schlieder theorem, $\widetilde{\Theta}=0$ if and only if $c=0$.

Proposition VI.3: The conformally covariant derivatives of the $U(1)$ current in $1+1$ dimensions do not contain a stress-energy tensor.

Proof: Looking at the commutation relations of the modes of $\Phi^{(n)}$ (equation VI.1), we learn that the eigenspaces of the conformal Hamiltonian $L_{0}$ associated with energy $1, \ldots, n$ are all null. If $n \geq 2$ this yields for $L_{-2}=\widetilde{\Theta}\left(\left[z^{-1}\right]\right): c / 2=$ $\left\|L_{-2} \Omega\right\|^{2}=0$, and hence $\widetilde{\Theta}=0$.

In the case $n=1$ all vectors of energy 2 are multiples of $\Phi_{-2}^{(1)} \Omega$. If there is a stress-energy tensor $\widetilde{\Theta}$ then we have: $\gamma L_{-2} \Omega=\Phi_{-2}^{(1)} \Omega, c|\gamma|^{2}=12$. Obviously, $\widetilde{\Phi}^{(1)}-\gamma \widetilde{\Theta}$ is a quasi-primary field and its two-point function is determined by conformal covariance up to a constant, $C \geq 0$ :

$$
\left\langle\Omega,\left(\widetilde{\Phi}^{(1)^{\dagger}}(z)-\bar{\gamma} \widetilde{\Theta}^{\dagger}(z)\right)\left(\widetilde{\Phi}^{(1)}(w)-\gamma \widetilde{\Theta}(w)\right) \Omega\right\rangle=C\left(z_{>}-w\right)^{-4} .
$$

In particular, we have: $C=\left\|\left(\Phi_{-2}^{(1)}-\gamma L_{-2}\right) \Omega\right\|^{2}=0$. By the REEH-SCHLIEDER theorem, the field $\widetilde{\Phi}^{(1)}-\gamma \widetilde{\Theta}$ is zero. Since $\gamma^{-1} \widetilde{\Phi}^{(1)}$ is not a stress-energy tensor, 
the claim holds for $n=1$ as well.

We have shown that the quantum field theory of the conformally covariant derivatives of the $U(1)$ current in $1+1$ dimensions does not contain a stress-energy tensor. This adds another detail to their character as archetypes of conformal theories in $1+1$ dimensions: In spite of being completely well-behaved, they do not exhibit special properties of other comparatively simple models such as strong additivity or presence of a stress-energy tensor.

If there is a local density associated in some sense with the conformal symmetry of these models, it has to be of a different nature. The proofs of the lemmas IV.2 and IV.12, which are the foundation of chapter IV, indicate why such densities are desirable. We discuss possible generalisations in the following chapter. 


\section{Chapter VII}

\section{Discussion}

\section{Summary}

We studied chiral conformal subnets $\mathcal{A} \subset \mathcal{B}$, their Coset models $\mathcal{C} \subset \mathcal{B}$, ie subnets of $\mathcal{B}$ which commute with $\mathcal{A}$, and found objects and structures intrinsically associated with the subnet $\mathcal{A} \subset \mathcal{B}$. Most of our results hold on general grounds and are not directly related to a specific class of models, although our work is inspired by current subalgebras and their CoseT models, compare chapter III. We abstracted from these examples when we formulated our general assumptions, see section II. 1.2 .

In section II.2, we found that there is a unique inner-implementing representation $U^{\mathcal{A}}$ with the following properties: $U^{\mathcal{A}}$ is affiliated with $\mathcal{A}$ in a global sense, its non-trivial unitaries are genuine global observables of the subnet $\mathcal{A}$. The adjoint action of $U^{\mathcal{A}}$ implements global conformal symmetry on the subnet $\mathcal{A} \subset \mathcal{B} . U^{\mathcal{A}}$ is a unitary, strongly continuous representation of $P S L(2, \mathbb{R})^{\sim}$ of positive energy which is complemented by another representation $U^{\mathcal{A}^{\prime}}$ by unitaries in the commutant of the global algebra $\mathcal{A}=\bigvee_{I \Subset \mathrm{S}^{1}} \mathcal{A}(I) . U^{\mathcal{A}^{\prime}}$ is a positive-energy representation of $P S L(2, \mathbb{R})^{\sim}$, too, and yields a factorisation of $U$, the implementation of global conformal symmetry in the vacuum representation of $\mathcal{B}$, as $U \circ \mathrm{p}=U^{\mathcal{A}} U^{\mathcal{A}^{\prime}}, \mathrm{p}: \operatorname{PSL}(2, \mathbb{R})^{\sim} \rightarrow \operatorname{PSL}(2, \mathbb{R})$ the covering projection. Both $U^{\mathcal{A}}$ and $U^{\mathcal{A}^{\prime}}$ leave the vacuum invariant.

$U^{\mathcal{A}}$ is intrinsic for the subnet $\mathcal{A} \subset \mathcal{B}$ and it is directly related to its CoseT structure: the local observables of $\mathcal{B}$ which commute with all unitaries of $U^{\mathcal{A}}$ form the maximal CoseT model $\mathcal{C}_{\text {max }}$; the local algebras of $\mathcal{C}_{\max }$ contain the local observables of all Coset models $\mathcal{C} \subset \mathcal{B}$ associated with the subnet $\mathcal{A} \subset \mathcal{B}$ (lemma II.4).

We raised the question whether COSET models are of a local nature, ie if there is an alternative characterisation not by global data like the unitaries of $U^{\mathcal{A}}$ but by local data, more specifically if the local algebras $\mathcal{C}_{\text {max }}(I)$ coincide with the local relative commutants $\mathcal{C}_{I}=\mathcal{A}(I)^{\prime} \cap \mathcal{B}(I)$. The task was to show that the 
$\mathcal{C}_{I}$ increase with the localisation region $I$ and we referred to this as the isotony problem. This problem exists for embeddings of theories in any spacetime and we argued that this problem must be taken seriously. We took a new perspective by studying it through the action of the inner-implementing representation $U^{\mathcal{A}}$ on the local observables of the ambient theory $\mathcal{B}$.

The construction of $U^{\mathcal{A}}$ relies on the fact that $P S L(2, \mathbb{R})^{\sim}$ is, as $\operatorname{PSL}(2, \mathbb{R})$ and all other global conformal groups [BGL93], generated by translation subgroups which fulfill the spectrum condition in every positive-energy representation of the global conformal group. A result of Borchers [Bor66] ensures the existence of globally inner implementations of automorphism groups of a V.NEumANn algebra $\mathcal{A}$ induced by the adjoint action of a positive-energy representation of a translation group. We derived from these implementations of translation automorphisms the globally inner implementation of all automorphisms associated with conformal symmetry. This construction generalises within its limits the SUGAWARA construction of stress-energy tensors for current algebras and we introduced the name Borchers-Sugawara construction for it since its key building block is the result of BORCHERS.

The Borchers-SugaWARA construction of $U^{\mathcal{A}}$ is completely general but it does not relate to the local structure of the chiral subnet. We found that a single Additional Assumption on the way $U^{\mathcal{A}}$ is generated by local observables of $\mathcal{A}$ (see page 65) is sufficient in order to determine the geometrical character of the adjoint action of $U^{\mathcal{A}}$ on local observables of $\mathcal{B}$ and to tackle the isotony problem: If $U^{\mathcal{A}}$ is generated by integrals of a stress-energy tensor contained in $\mathcal{A}$ both problems can be solved.

So, our analysis applies directly to a large class of examples, the current subalgebras and their COSET models which contain the CosET stress-energy tensor, because the SUGAWARA construction yields stress-energy tensors as explicit quadratic functions of the currents of the respective current algebra (theorem III.3). The isotony problem is of interest for this class of examples because the COSET models are not known to be strongly additive in general.

Even in presence of a stress-energy tensor we needed to pin down the problems of determining the geometric impact of $U^{\mathcal{A}}$ on local observables of $\mathcal{B}$ and of establishing isotony for the local relative commutants by general arguments. The Additional Assumption was only needed to establish two crucial, but natural lemmas (lemma IV.2 and lemma IV.12). Moreover, we did not use specific structures of current subalgebras, as the LÜSCHER-MACK theorem shows that the structures we need hold for any subtheory $\mathcal{A}$ which contains a stress-energy tensor provided the action of the stress-energy tensor on the vacuum HILBERT space of $\mathcal{B}$ has some weak technical properties (cf section III,1.2 and proof of theorem III.3).

Chapter IV contains the main results of this work: We established on grounds of the Additional Assumption that the adjoint action of dilatations $U^{\mathcal{A}}(\tilde{D}(t))$, $t \in \mathbb{R}$, induces automorphisms of $\mathcal{B}\left(\mathrm{S}_{+}^{1}\right)$ (lemma IV.2) and that this feature, which 
we call net-endomorphism property, allows a satisfactory analysis of the geometric impact of $U^{\mathcal{A}}$ on local observables of the ambient theory $\mathcal{B}$ (proposition IV.6). In particular, this ensures that for given $I \Subset \mathrm{S}^{1}$ the algebras $A d_{U^{\mathcal{A}}(\tilde{g})} \mathcal{B}(I)$ consist of local operators as long as $\tilde{g}$ is sufficiently close to the identity, in spite of the $U^{\mathcal{A}}(\tilde{g})$ being genuine global observables for $\tilde{g} \neq i d$. This was achieved through a converse of BORCHERS' theorem on half-sided translations (theorem IV.3), ie by an interplay of positivity of energy and modular theory.

The net-endomorphic action of $U^{\mathcal{A}}$ on $\mathcal{B}$ found its interpretation by chiral holography: $U^{\mathcal{A}}$ and $U^{\mathcal{A}^{\prime}}$ may be taken to be implementations of chiral conformal transformations on either light-cone coordinate in $1+1$ dimensions which generate from the chiral conformal theory $\mathcal{B}$ a $1+1$-dimensional holographic extension $\mathcal{B}^{1+1}$. The original net $\mathcal{B}$ is contained as the net of observables associated with timezero double cones, and $\mathcal{B}^{1+1}$ fulfills all properties of a conformal theory in $1+1$ dimensions with one exception: it's not the translations in futurelike directions but in right spacelike directions which have positive generators (theorem IV.7). For this reason we called $\mathcal{B}^{1+1}$ a quasi-theory.

$\mathcal{B}^{1+1}$ allows to take a geometrical point of view with respect to chiral subnets and their Coset models: the maximal Coset model $\mathcal{C}_{\text {max }}$ and the chiral subnet $\mathcal{A}_{\text {max }}$, consisting of all local observables of $\mathcal{B}$ on which $U^{\mathcal{A}}$ implements conformal covariance, are contained in $\mathcal{B}^{1+1}$ as subtheories of all left, respectively right chiral observables. This establishes a direct connection between chiral subnets and their CoseT models and inclusions of chiral observables in 1+1-dimensional conformal theories as studied before by REHREN [Reh00].

Chiral holography shows that the symmetries which yield chiral subnets and their COSET models as fixed-point subtheories is a spacetime symmetry given by the chiral conformal transformations on the respective other light-cone coordinate. There is no need to broaden the symmetry concept in order to understand these inclusions, one only has to go into the chirally holographic picture.

The interpretation of $\mathcal{A}_{\max }, \mathcal{C}_{\max }$ as chiral observables in $\mathcal{B}^{1+1}$ directly leads to a spatial identification of these subnets: the HILBERT subspaces they generate from the vacuum coincide with the spaces of $U^{\mathcal{A}^{\prime}}$ - and $U^{\mathcal{A}}$-invariant vectors, respectively [Reh00, lemma 2.3]. We gave an alternative proof using the netendomorphism property of $U^{\mathcal{A}}$ directly and showed that the subnet generated by a chiral subnet $\mathcal{A} \subset \mathcal{B}$ and any Coset model, $\mathcal{C}$, is the tensor product $\mathcal{A} \otimes \mathcal{C}$ of both theories (proposition IV.8). This has interesting applications (see below).

In section IV 3 we solved the isotony problem in our setting. The ADDitionaL Assumption was used to establish that the dilatations $U^{\mathcal{A}}(\tilde{D}(t)), t \in \mathbb{R}$, are contained in the algebra $\mathcal{A}\left(\mathrm{S}_{+}^{1}\right) \vee \mathcal{A}\left(\mathrm{S}_{-}^{1}\right)$ (lemma IV.12). After being complemented with the net-endomorphism property and some general arguments this proved sufficient to establish isotony for the local relative commutants; we summarised this in our main result, theorem IV.13.

On the way to our solution of the isotony problem we used structures of the chiral conformal group, $\operatorname{PSL}(2, \mathbb{R})$, and of the group of orientation preserv- 
ing diffeomorphisms of the circle, Diff $\left(\mathrm{S}^{1}\right)$, or rather their universal covering groups and of their (exponentiated) positive-energy representations. The latter group comes into play by the theorem of LÜSCHER AND MACK [FST89, Mac88, LM76] on the commutation relations of stress-energy tensors in chiral and 1+1dimensional conformal quantum field theory. A technical result of ToLEDANOLAREDO [TL99] on the exponentiation of these commutation relations was vital for proving the ADDITIONAL Assumption in presence of stress-energy tensors (compare proof of theorem III.3).

Our approach is independent of the properties of strong additivity and finiteness of index for $\mathcal{A} \subset \mathcal{C}_{\text {max }}(\mathcal{A} \subset \mathcal{B}$ cofinite $)$, which are central for other studies on chiral subnets and their COSET models and which may be established for current subalgebras by taking advantage of their specific structure, compare in particular [Xu00a, Xu99], [Lon01], [KL02], [Lok94]. Both approaches are complementary, and our analysis of the action of the inner-implementation $U^{\mathcal{A}}$ on the ambient theory $\mathcal{B}$ yields new insights for cofinite inclusions of current algebras as well:

The spatial identification of $\mathcal{A}_{\max }$ and $\mathcal{C}_{\max }$ (proposition IV.8) allowed us interpret branchings for particular current subalgebras $\mathcal{A} \subset \mathcal{B}$ and concrete CosET models $\mathcal{C}$ which are available from group-theoretic analysis [GKO86, KW88, KNS88] as restricted branchings of normal canonical tensor product subfactors (CTPS) [Reh00] given by $\mathcal{A}_{\max } \subset \mathcal{C}_{\max } \subset \mathcal{B}$. We gave a Coset construction of the third exceptional local extension of $\mathcal{V} i r_{c<1}$ models as classified in [KL02] (section V.3.2) and found a normal CTPS in an ambient theory with trivial superselection structure, $\mathcal{L} E(8)_{1}$, a setting discussed by MüGER [Müg02] (see section $\mathrm{V}, 3.3)$.

We obtained some results for general cofinite subnets $\mathcal{A} \subset \mathcal{B}$ as well: in this setting the net-endomorphism property alone is sufficient to solve the isotony problem (proposition V.2). Moreover, for Coset pairs $\mathcal{A} \subset \mathcal{C} \subset \mathcal{B}$ which are spatial (definition V.9) the chiral rigid rotations by $2 \pi, U^{\mathcal{A}}(\tilde{R}(2 \pi))$, induce automorphisms of each local algebra $\mathcal{B}(I)$ (proposition V.11), which improves the general knowledge on the net-endomorphic action of $U^{\mathcal{A}}$ on $\mathcal{B}$ (cf proposition IV.6).

We found that for spatial Coset pairs $\mathcal{A} \subset \mathcal{C} \subset \mathcal{B}$ of finite index the quasitheory $\mathcal{B}^{1+1}$ can fulfill timelike commutativity and hence can be reinterpreted directly as a genuinely physical model only if the spectrum of $U^{\mathcal{A}}(\tilde{R}(2 \pi))$ is contained in $\{ \pm 1\}$ (proposition V.12). This necessary condition excludes sharp geometrical action of $U^{\mathcal{A}}$ on the chiral theory $\mathcal{B}$, which is equivalent with timelike commutativity of $\mathcal{B}^{1+1}$ (proposition IV.10), for all (known) current subalgebras except the cases one can make up trivially.

In section III. 4 we applied our new perspective of analysing the action of the inner-implementation of a subtheory on the observables of the ambient theory when we gave a direct characterisation of conformal inclusions of current algebras. Moreover, one may recognise from this discussion that it is of limited use to know explicitly the commutation relations of the stress-energy tensor of a subtheory 
on the fields of the ambient theory, which we derived for current subalgebras (equation III.42).

Chapter VI, finally, was to show that there are well-behaved conformal models which do not possess a stress-energy tensor, namely the conformally covariant derivatives of the $U(1)$ current. Since these models live in $1+1$ dimensions but decompose into independent chiral components, they provide examples of this structure in both settings. If there is a local density associated with the conformal symmetry of these models, it has to be of a different nature, and this asks for possible generalisations of our discussion.

\section{Outlook}

A very general quantum version of NOETHER's theorem exists [BDL86] on grounds of the split property, which was established easily for the conformally covariant derivatives in section VI.1. Here, symmetries are implemented on local algebras by operators which are localised in a somewhat enlarged region. CARPI [Car99] took point-like limits of local implementers applying methods of JöRSS AND FREDENHAGEN [FJ96] and reconstructed the stress-energy tensor of some models in this way. Hence he gave an explicit account of the relation of local implementers to densities as needed in chapter IV. We comment briefly of the possible application of local implementers in the context of chiral subnets and the isotony problem.

Let us begin with a short summary of the quantum NoETHER theorem of Buchiolz, Doplicher And Longo [BDL86]. The split property of the subnet $\mathcal{A}$, which definitely holds if $\mathcal{B}$ is split (proposition II.7), proves that for given $I \Subset \mathrm{S}^{1}$ and each $J \Subset \mathrm{S}^{1}$ which contains the closure of $I$ there is a faithful, normal product state $\eta_{I \subset J}$ for $\mathcal{A}(I) e_{\mathcal{A}} \vee \mathcal{A}\left(J^{\prime}\right) e_{\mathcal{A}}$ [Buc74]. This state is then taken to define the universal localisation map $\Psi_{I \subset J}$ which maps each bounded operator on $e_{\mathcal{A}} \mathscr{H}$ to a local observable in $\mathcal{A}(J) e_{\mathcal{A}}$ and acts trivially on $\mathcal{A}(I) e_{\mathcal{A}}$. The universal localisation map is a $*$-homomorphism of norm 1 and the operators $\Psi_{I \subset J}\left(U(g) e_{\mathcal{A}}\right), g \in P S L(2, \mathbb{R})$, form a representation of $P S L(2, \mathbb{R})$ with the same spectral properties as $U e_{\mathcal{A}}$. For $g \in P S L(2, \mathbb{R})$ and $I_{0} \Subset \mathrm{S}^{1}$ which satisfy $g I_{0} \subset I$, the adjoint action of $\Psi_{I \subset J}\left(U(g) e_{\mathcal{A}}\right)$ implements the automorphism $\alpha_{g}$ on $\mathcal{A}\left(I_{0}\right) e_{\mathcal{A}}$, and for this reason the operators $\Psi_{I \subset J}\left(U(g) e_{\mathcal{A}}\right)$ are called local implementers.

By local normality of the embedding $\mathcal{A} \subset \mathcal{B}$ we can lift the local implementers from the vacuum subrepresentation to the subnet, and we write the corresponding operator in $\mathcal{A}(J)$ by $\Psi_{I \subset J}^{\mathcal{A} \subset \mathcal{B}}\left(U(g) e_{\mathcal{A}}\right)$. These operators are local implementers for the subtheory in the obvious sense. Taking advantage of the group structure of $\operatorname{PSL}(2, \mathbb{R})$ one can use the locality of $\mathcal{B}$ to show that every local observable of $\mathcal{B}\left(I_{0}\right)$ which commutes with the local implementers $\Psi_{I \subset J}^{\mathcal{A} \subset \mathcal{B}}\left(U(g) e_{\mathcal{A}}\right)$ for some neighbourhood of the identity commutes with all of $\mathcal{A}\left(I_{0}\right)$. This allows a characterisation of the maximal CoseT model analogous to the one given in lemma 


\section{II.4.}

If we want to deal with the isotony problem from this angle, however, we have to look at the limit $J \rightarrow I_{0}$, and it seems unlikely that it might prove possible to establish non-trivial weak limit points for the local implementers themselves for $g \neq i d$. But looking at the proof of lemma IV.12, equation (IV.11) in particular, one might hope that such limit points exist for bilocal products of local implementers as

$$
\Psi_{I_{-} \subset J_{-}}^{\mathcal{A} \subset \mathcal{B}}\left(U\left(g_{-}\right) e_{\mathcal{A}}\right) \Psi_{I_{+} \subset J_{+}}^{\mathcal{A} \subset \mathcal{B}}\left(U\left(g_{+}\right) e_{\mathcal{A}}\right), I_{ \pm}, J_{ \pm} \subset S_{ \pm}^{1}, g_{ \pm} \in P S L(2, \mathbb{R}) .
$$

in the limit $I_{ \pm} \rightarrow \mathrm{S}_{ \pm}^{1}, g_{ \pm} \rightarrow D(t) \neq i d$ (for small $t$ ). To establish such limit points one has to relate the product states to the subnet structure. In case there are weak limit points which agree with $U^{\mathcal{A}}(\tilde{D}(t)$ up to a phase (cf equation (IV.11)), one directly gets a solution for the isotony problem by the arguments in the proof of lemma IV.12.

It was proved in [DDFL87] that there are choices for the enlarged regions (intervals $J$ above) such that the universal localisation maps converge point wise strongly to the identity map in the limit of the localisation regions exhausting spacetime (in our case $I$ tending to $\mathbb{R}$ ), provided the union of all local algebras acts irreducibly on HILBERT space [DDFL87]; but there is no method available to establish weak limit points for bilocal operators of a subtheory.

We end our speculations on the possible role of local implementers in the context of chiral subnets with a simple observation: Provided one can establish weak limit points for bilocal operators as in (VII.1) which agree with $U^{\mathcal{A}}(\tilde{D}(t))$ for $t \neq 0$ up to a phase and weak limit points for corresponding local implementers of the ambient theory $\mathcal{B}$ which agree with the dilatations $U(D(t))$ up to a phase, it is clear that $U^{\mathcal{A}^{\prime}}(\tilde{D}(t))=U(D(t)) U^{\mathcal{A}}(\tilde{D}(t))^{*}$ is affiliated with the maximal Coset model.

If one uses the representatives of localised diffeomorphisms instead of local implementers, such a connection is established by (a corresponding version of) equation (IV.11), if both $\mathcal{A}$ and $\mathcal{B}$ contain a stress-energy tensor. In fact, if $U^{\mathcal{A}^{\prime}}(\tilde{D}(t))$ is affiliated with $\mathcal{C}_{\text {max }}$, covariance directly leads to the identity $U^{\mathcal{A}^{\prime}}=U^{\mathcal{C}_{\text {max }}}$. Hence, the isotony problem appears to be intimately related to the conformal inclusion problem which we discussed in section II.2.2 (compare proposition V.2 for a similar connection for cofinite subnets).

It is the author's opinion that one should start investigating the relation of local implementers to the problems outlined above by investigating possible weak limit points of bilocal products of local implementers in the vacuum representation of the conformally covariant derivatives of the $U(1)$ current. These are particularly simple models as they satisfy canonical commutation relations and the split property was established very easily for these models (section VI.1).

Finally, we comment briefly on possible generalisations of our analysis to subtheories in other spacetimes: Our analysis directly generalises to $1+1$ dimensions, 
because in this context any stress-energy tensor decomposes into two independent chiral parts (by the LÜSCHER-MACK theorem), the global conformal group is a factor group of $\operatorname{PSL}(2, \mathbb{R})^{\sim} \times \operatorname{PSL}(2, \mathbb{R})^{\sim}$, and double cones and their causal complements in 1+1-dimensional covering space are Cartesian products of proper intervals on the (simply connected covering of the) circle.

Adaptations of the Borchers-SugaWARA construction for conformal groups of higher-dimensional spacetimes are possible (see discussion in section II 2.3) and provided one can establish the corresponding versions of the two crucial lemmas (lemma IV.2, lemma IV.12) our general strategy will work because it only involves general properties of conformal groups and the causal structure of conformal coverings of spacetime (cf [BGL93]). Since there is no equivalent of the LÜSCHER-MACK theorem in higher dimensions, one probably has to use local implementers in order to establish substitutes of the lemmas IV.2 and IV.12.

For subnets with lesser spacetime symmetry like POINCARÉ invariant theories in $3+1$ dimensions one has to resort to different methods. In this context, CARPI AND CONTI solved the isotony problem by methods less direct than ours, but very general ones $[\mathrm{CC} 01, \mathrm{CC}]$. Thus, the quest for the heart of this problem still awaits further investigation. 


\section{Appendix A}

\section{Lemmas on $\operatorname{PSL}(2, \mathbb{R})$ and $\operatorname{PSL}(2, \mathbb{R})^{\sim}$}

This appendix contains some statements on the group of global chiral conformal transformations, $P S L(2, \mathbb{R})$, and its universal covering group, $P S L(2, \mathbb{R}) \sim$. For a short summary on the properties of the group $P S L(2, \mathbb{R})$ and its action on $\mathrm{S}^{1}$ see [GF93]; a very short introduction is contained in section II.1.1.

The following lemma is crucial for the solution of the isotony problem (theorem IV.13) and it is involved in the proof of proposition IV.8 as well. Its statement is a variation of lemma B.2 in [GL96] and its proof is a detailed version of the argument indicated by GuIDO AND Longo.

Lemma A.1: Let $\mathscr{H}$ be a separable HILBERT space and $V$ a unitary, strongly continuous representation of $P S L(2, \mathbb{R})^{\sim}$ on $\mathscr{H}$. If $H \subset P S L(2, \mathbb{R})^{\sim}$ is a subgroup having closed, non-compact image in $P S L(2, \mathbb{R})$ under the action of the covering projection $\mathrm{p}$, then each $\left.V\right|_{H}$-invariant vector is in fact $V$-invariant. If $V$ is a representation of positive energy, then each vector which is invariant with respect to $V(\tilde{R}()$.$) is V$-invariant as well.

Proof: As a first step we reduce $V$ by its trivial subrepresentation, $\mathbb{1}_{\mathscr{H}_{0}}$, and $\mathscr{H}$ by the Hilbert subspace of $V$-invariant vectors, $\mathscr{H}_{0}$. For any vector in $\mathscr{H}_{0}$ the statements of the lemma are true, obviously, and we only have to discuss the complementary representation $V^{\perp}$ on the HILBERT subspace orthogonal to $\mathscr{H}_{0}$,

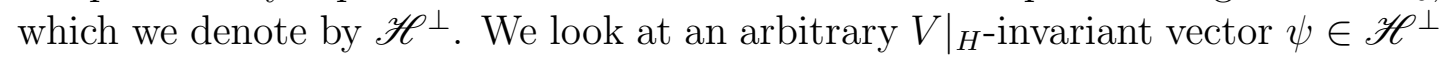
and prove that any such vector has to vanish. A technical complication arises as $P S L(2, \mathbb{R})^{\sim}$ has an infinite centre. This forces us to apply a trick included in the proof of [GL96, corollary B.2.].

We decompose $V^{\perp}$ into a direct integral of irreducible representations $V_{x}$ (see eg [BR77]). The corresponding components $\psi_{x}$ of $\psi$ are almost all $\left.V_{x}\right|_{H^{-}}$invariant and almost all $V_{x}$ are different from the trivial representation. Next, we take the tensor product of a non-trivial $V_{x}$ and its conjugate representation, $\overline{V_{x}}$. We prove: $V_{x} \otimes \overline{V_{x}}$ does not contain the trivial representation. To this end we assume that there is a partial isometry $W: \mathscr{H}_{x} \otimes \overline{\mathscr{H}_{x}} \rightarrow \mathbb{C}$ intertwining $V_{x} \otimes \overline{V_{x}}$ and the 
trivial representation on $\mathbb{C}$. Using [KR83] (theorem 2.4.1) we define an operator $T: \mathscr{H}_{x} \rightarrow \mathscr{H}_{x}$ with $\|T\| \leq 1$ by $\left\langle\eta_{2}, T \eta_{1}\right\rangle_{x}:=W \eta_{1} \otimes \overline{\eta_{2}}, \eta_{1,2} \in \mathscr{H}_{x}$. One readily checks that $T$ commutes with $V_{x}$ and hence is of the form $e^{i \varphi}\|T\| \mathbb{1}$. Choosing an orthonormal basis $\left\{e_{n}\right\}_{n \in \mathbb{N}}$ this yields for $\chi_{N}:=\sum_{n=1}^{N} \frac{1}{n} e_{n} \otimes \overline{e_{n}}$ the following: $W \chi_{N}=e^{i \varphi}\|T\| \sum_{n=1}^{N} \frac{1}{n}$. Since $\mathscr{H}_{x}$ has infinite dimension [Gri93] this contradicts the assumption: $\lim _{N \rightarrow \infty} \chi_{N} \in \mathscr{H}_{x} \otimes \overline{\mathscr{H}}_{x}$ but $W \chi_{N}$ is unbounded.

We know that $V_{x} \otimes \overline{V_{x}}$ does not admit a non-trivial invariant vector and it forms a representation of $\operatorname{PSL}(2, \mathbb{R})$ as the kernel of $V_{x} \otimes \overline{V_{x}}$ contains the centre of $\operatorname{PSL}(2, \mathbb{R})^{\sim}$. Now we are in the position to apply [Zim84, theorem 2.2.20] and thus we have for any $\xi_{x} \in \mathscr{H}_{x}$ :

$$
\lim _{\mathbf{p}(\tilde{g}) \rightarrow \infty}\left|\left\langle V_{x}(\tilde{g}) \xi_{x}, \xi_{x}\right\rangle\right|^{2}=\lim _{\mathbf{p}(\tilde{g}) \rightarrow \infty}\left\langle\left(V_{x} \otimes \overline{V_{x}}\right)(\tilde{g}) \xi_{x} \otimes \overline{\xi_{x}}, \xi_{x} \otimes \overline{\xi_{x}}\right\rangle=0 .
$$

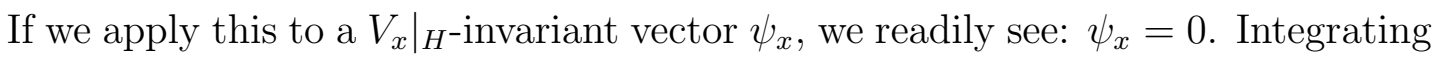
over $x$ yields the first statement of the lemma.

The result on rigid conformal rotations may be deduced in the same manner: The irreducible representations $V_{x}$ are almost all of positive energy and the only irreducible representation of $\operatorname{PSL}(2, \mathbb{R})^{\sim}$ having positive energy and containing a non-trivial $\tilde{R}($.$) -invariant vector is the trivial representation [Gri93].$

The following lemma is a variant of [GL96, lemma B.3]:

Lemma A.2: $V$ a unitary, strongly continuous representation of the translationdilatation group on a HILBERT space $\mathscr{H}$. Then every finite-dimensional subspace which is left invariant globally by $V(D(\tau))$ for some $\tau \neq 0$ consists of translation invariant vectors.

Proof: Let $\mathscr{K}$ denote the finite-dimensional subspace of $\mathscr{H}$ left invariant by $V(D(\tau))$. Then $V(D(\tau))$ may be diagonalised on $\mathscr{K}$ and we take some eigenvector $\psi$ satisfying $V(D(\tau)) \psi=a \psi$. We treat the case $\tau>0$, else one has to look at $V(D(\tau))^{*} \psi=a^{-1} \psi$ :

$$
\langle\psi, V(T(t)) \psi\rangle=\left\langle a^{-1} V(D(\tau)) \psi, V(T(t)) a^{-1} V(D(\tau)) \psi\right\rangle=\left\langle\psi, V\left(T\left(e^{-\tau} t\right)\right) \psi\right\rangle
$$

$n$-fold iteration yields the identity $\langle\psi, V(T(t)) \psi\rangle=\langle\psi, \psi\rangle$ in the limit $n \rightarrow \infty$. It is elementary to check that this implies $\|\psi-V(T(t)) \psi\|^{2}=0$, ie $\psi=V(T(t)) \psi$.

The identity components $S O(s, 1)_{+}^{\uparrow}$ of the LoRENTZ groups in $s+1$ dimensions, $S O(s, 1)$, are known to be exponential, ie the exponential map is surjective for these groups [Mos94, Mos97]. This applies to $P S L(2, \mathbb{R}) \cong S O(2,1)_{+}^{\uparrow}$, but we consider it worthwhile to give a straightforward elementary proof.

Proposition A.3: Every element in $P S L(2, \mathbb{R})$ lies on at least one one-parameter subgroup. 
Proof: We solve the problem using the formulation of $\operatorname{PSU}(1,1) \cong P S L(2, \mathbb{R})$ as automorphisms of the open unit disc $D_{1} \subset \mathbb{C}$ (see eg [FL80]). Such automorphisms, $f$, are parametrised in terms of their zero, $z_{0}$, and a complex phase, $e^{i \phi}$ :

$$
f(z)=e^{i \phi} \frac{z-z_{0}}{1-\overline{z_{0}} z}, z \in D_{1} .
$$

It easy to see, that the action of $P S U(1,1)$ on $\mathrm{S}^{1}$, equation (II.3), is exactly of this form; the inverse $f^{-1}$ of an automorphism $f$ has inverse phase, $e^{-i \phi}$. Hence, we may restrict attention to $0 \leq \phi \leq \pi$.

We will prove that it is possible to take the square root of every automorphism of $D_{1}$ and that iterating this process eventually yields an $n$th root arbitrarily close to the identity $\left(z_{0}=0, e^{i \phi}=1\right)$. In a neighbourhood of the identity the exponential map is known to be a (local) diffeomorphism from the LIE algebra into the group (eg [HN91, Korollar III.2.10]).

Given $f$, we seek a $g$, parametrised by $w_{0}, e^{i \psi}$, which solves $f=g^{2}$ and which is closer to the identity. $g$ has to solve the following equations:

$$
\begin{aligned}
& \frac{w_{0}\left(e^{i \psi}+1\right)}{e^{i \psi}+\left|w_{0}\right|^{2}} \stackrel{!}{=} z_{0}, \\
& \frac{e^{i \psi}\left(e^{i \psi}+\left|w_{0}\right|^{2}\right)}{1+\left|w_{0}\right|^{2} e^{i \psi}-z \overline{w_{0}}\left(1+e^{i \psi}\right)} \stackrel{!}{=} \frac{e^{i \phi}}{1-\overline{z_{0}} z}, \quad z \in D_{1} .
\end{aligned}
$$

The case $e^{i \phi}=1$ leads to elementary quadratic equations whose solutions have again $e^{i \psi}=0$. The iterated square roots can readily be seen to tend towards the identity. Solutions satisfying $w_{0}=z_{0}$ can only occur for $z_{0}=0$; in this case $f$ is a pure rotation and lies on a one-parameter group.

In the other cases, one can derive the following, equivalent pair of equations for $w_{0}, e^{i \psi}$ :

$$
\begin{aligned}
& w_{0}=e^{-i \frac{\psi}{2}} e^{i \frac{\phi}{2}} z_{0} \frac{\sin \frac{\psi}{2}}{\sin \frac{\phi}{2}}, \\
& \sin \left(\frac{\phi}{2}-\psi\right)\left|1-e^{i \phi}\right| \stackrel{!}{=}(1-\cos \psi)\left|z_{0}\right|^{2} .
\end{aligned}
$$

Looking at the possible graphs of the functions equated in the second formula one readily sees that there always is a solution with $\psi<\frac{\phi}{2}$. Taking this solution, one immediately gets $w_{0}$ with $\left|w_{0}\right|^{2}<\frac{1}{2}\left|z_{0}\right|^{2}$. Again, this is just what we need.

The group $S L(2, \mathbb{R})$ can easily be seen not to be exponential: there is no $g \in S L(2, \mathbb{R})$ solving the equation $\operatorname{diag}\left(-e^{\tau},-e^{-\tau}\right)=g^{2}$. 


\section{Lemmas on $\operatorname{Diff}_{+}\left(\mathrm{S}^{1}\right)$ and $\operatorname{Diff}_{+}\left(\mathrm{S}^{1}\right)^{\sim}$}

This appendix contains some technical lemmas on the group of orientation preserving diffeomorphisms of the circle, Diff $\left(\mathrm{S}^{1}\right)$, and its universal covering group, Diff $_{+}\left(\mathrm{S}^{1}\right)^{\sim}$. For short summaries on its general properties and more statements related to local quantum physics see [Mil84, PS86, Lok94].

The first lemma is a simple statement on the position of scale transformations in Diff $\left(\mathrm{S}^{1}\right)^{\sim}$; it is needed in the proofs of lemma IV.2 and lemma IV.12.

Lemma A.4: For a fixed scale transformation $D(t) \neq i d, t$ small, there exist diffeomorphisms $g_{\delta}, g_{\varepsilon} \in D$ iff ${ }_{+}\left(\mathrm{S}^{1}\right)$ which are localised in arbitrarily small neighbourhoods of +1 and -1 , respectively, and which agree with $D(t)$ close to +1 and -1 , respectively, such that, by defining

$$
g_{\delta}^{\tau_{1}}:=D\left(\tau_{1}\right) g_{\delta} D\left(\tau_{1}\right)^{-1}, g_{\varepsilon}^{\tau_{2}}:=D\left(\tau_{2}\right)^{-1} g_{\varepsilon} D\left(\tau_{2}\right)
$$

we have for all $\tau_{1,2} \in \mathbb{R}_{+}$:

$$
D(t)=g_{+}^{\tau_{1}, \tau_{2}} g_{-}^{\tau_{1}, \tau_{2}} g_{\delta}^{\tau_{1}} g_{\varepsilon}^{\tau_{2}}
$$

Here, the diffeomorphisms $g_{+}^{\tau_{1}, \tau_{2}}, g_{-}^{\tau_{1}, \tau_{2}}$ are uniquely specified by their being localised in the upper and lower half circle, respectively. After a local identification of Diff $\left(\mathrm{S}^{1}\right)$ with a sheet of Diff $\left(\mathrm{S}^{1}\right)^{\sim}$ containing the identity, equation (A.1) still holds for the respective images in Diff $\left(\mathrm{S}^{1}\right)^{\sim}$.

Proof: If $I_{1}$ and $I_{2}$ are neighbouring intervals, the "completed union" which consists of $I_{1} \cup I_{2}$ and the common boundary point will be denoted $I_{1} \cup I_{2}$.

Choose a set $\left\{I_{\iota}^{0}, \iota=+,-, \delta, \varepsilon\right\}$ of proper, disjoint intervals such that $I_{ \pm}^{0} \subset \mathrm{S}_{ \pm}^{1}$, $+1 \in I_{\delta}^{0},-1 \in I_{\varepsilon}^{0}$, the $I_{\iota}^{0}$ are separated by proper intervals $I_{a}, . ., I_{d}$ and a covering of $\mathrm{S}^{1}$ by proper intervals $I_{\iota}^{1}$ is defined through:

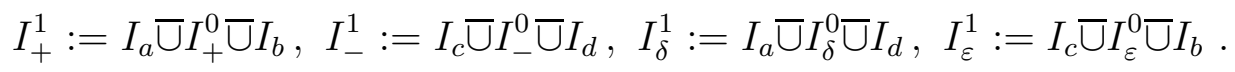

For fixed $t$, one can choose these intervals such that $D(t)$ satisfies $D(t) I_{\iota}^{0} \Subset I_{\iota}^{1}$. Since $D(t) \mathrm{S}_{ \pm}^{1} \subset \mathrm{S}_{ \pm}^{1}$, we may choose $g_{\delta}, g_{\varepsilon}$ close to $i d$ such that $g_{\delta}$ agrees with $D(t)$ on $I_{\delta}^{0}$ and with $i d$ on $I_{\delta}^{1 \prime}$ and $g_{\varepsilon}$ agrees with $D(t)$ on $I_{\varepsilon}^{0}$ and with $i d$ on $I_{\varepsilon}^{1 \prime}$. Referring to this choice we set:

$$
g_{ \pm} \uparrow I_{ \pm}^{1}:=D(t) g_{\delta}^{-1} g_{\varepsilon}^{-1} \uparrow \mathrm{S}_{ \pm}^{1}, \quad g_{ \pm} \uparrow \overline{\mathrm{S}_{\mp}^{1}}:=i d \uparrow \overline{\mathrm{S}_{\mp}^{1}}
$$

Then we have $D(t)=g_{+} g_{-} g_{\delta} g_{\varepsilon}$. We may now apply the definitions in the lemma to this choice and recognise the results to satisfy equally well the assumptions of the construction just given.

For a neighbourhood of the identity the covering projection $\mathrm{p}:$ Diff $\left(\mathrm{S}^{1}\right)^{\sim} \rightarrow$ $\operatorname{Diff}_{+}\left(\mathrm{S}^{1}\right)$ is a homeomorphism. If we apply $\mathrm{p}^{-1}$ to $D(t), g_{\delta}, g_{\varepsilon}, g_{+}, g_{-}$, we have 
$\mathrm{p}^{-1}(D(t))=\mathrm{p}^{-1}\left(g_{+}\right) \mathrm{p}^{-1}\left(g_{-}\right) \mathrm{p}^{-1}\left(g_{\delta}\right) \mathrm{p}^{-1}\left(g_{\varepsilon}\right)$. For small $\tau_{1}, \tau_{2}$ the equality (A.1) holds with the corresponding replacements, and the same is true for all $\tau_{1,2} \in \mathbb{R}_{+}$ by continuity: denoting the covering projection from $\mathbb{R}$ onto $S^{1}$ by $p$, all the group elements involved belong to the identity component of the subgroup of Diff $_{+}\left(\mathrm{S}^{1}\right)^{\sim}$ which stabilises $\mathrm{p}^{-1}(+1)$ and $\mathrm{p}^{-1}(-1)$, ie we never leave the first sheet of the covering.

Basically, the following lemma says: For all elements $\phi$ in a neighbourhood of the identity in Diff $\left(\mathrm{S}^{1}\right)$ there is a presentation as a finite product of localised diffeomorphisms $\Xi_{i}(\phi)$, which are continuous and unital functions of the group element $\phi$. Its general ideas are due to D'Antoni and Fredenhagen 1 1.

Lemma A.5: Let $\left\{I_{i}\right\}_{i \in \mathbb{Z}_{m}}$ be a finite covering of the circle by three or more proper intervals having the following additional properties: $I_{i} \cap I_{i+1}=: I_{i, i+1} \Subset \mathrm{S}^{1}$, $I_{i} \cap I_{j}=\varnothing$ if $j \notin\{i \pm 1, i\}$.

We choose a neighbourhood $\mathcal{U}_{\varepsilon} \subset$ Diff $\left(\mathrm{S}^{1}\right)$ containing the identity and depending on $\varepsilon>0,1>\delta>0$, such that for all $\phi \in \mathcal{U}_{\varepsilon}$ the following conditions are fulfilled:

$$
\begin{array}{rrrl}
(i): & \min _{z \in \mathrm{S}^{1}}\left\{[\arg \phi(z)]^{\prime}\right\} & >\delta, \\
(\text { ii }): & & |\arg \phi(z)-\arg z| & <\varepsilon \delta .
\end{array}
$$

Then there are $\varepsilon$ such that there exist continuous localisation mappings $\Xi_{i}$ : $\mathcal{U}_{\varepsilon} \longrightarrow \operatorname{Diff}_{\widetilde{I}_{i}}\left(\mathrm{~S}^{1}\right), \widetilde{I}_{i} \Subset \mathrm{S}^{1}, i=1, . ., m$, with the following features:

$$
\phi=\prod_{i=1}^{m} \Xi_{i}(\phi), \quad \Xi_{i}(i d)=i d .
$$

Proof: Equivalently we look at periodic diffeomorphisms of the real axis: $\varphi \in$ $C^{\infty}(\mathbb{R}), \varphi^{\prime}(x)>0, \varphi(x+2 \pi)=\varphi(x)+2 \pi$. We denote the analogue of $\varphi$ in $\operatorname{Diff}_{+}\left(\mathrm{S}^{1}\right)$ by $\check{\varphi}$. The preimage of an interval $I \Subset \mathrm{S}^{1}$ under the covering projection $\mathrm{p}$ will be called $\hat{I}$. We choose a smooth partition $\mu$ of unity on $\mathrm{S}^{1}$ satisfying $1 \geq \mu_{i} \geq 0, \operatorname{supp}\left(\mu_{i}\right) \subset I_{i}$. On the covering space we define $\lambda_{i}(x):=\mu_{i}(\mathrm{p}(x))$.

We set: $\Psi_{k}[\varphi](x):=x+\sum_{i=1}^{k} \lambda_{i}(x)(\varphi(x)-x), k=0, \ldots, m . \Psi_{k}[\varphi]$ coincides with $\varphi$ on $\hat{M}_{k}:=\left\{\bigcup_{j=k+1}^{m} I_{j}\right\}^{\prime}$ and with $i d$ on $\hat{N}_{k}:=\left\{\bigcup_{j=1}^{k} I_{j}\right\}^{\prime}$, since the sum $\sum_{i=1}^{k} \lambda_{i}($.$) takes the values 1$ and 0 , respectively. On $\hat{I}_{m, 1}$ we have (corresponding bound for $\left.\hat{I}_{k, k+1}\right)$ :

$$
\begin{aligned}
\Psi_{k}[\varphi]^{\prime}(x) & =\lambda_{m}(x)+\lambda_{1}(x) \varphi^{\prime}(x)+\lambda_{1}^{\prime}(x)(\varphi(x)-x) \\
& \geqslant \min \left\{1, \varphi^{\prime}(x)\right\}-\max _{\xi \in \hat{I}_{m, 1}}\left|\lambda_{1}^{\prime}(\xi)\right||\varphi(x)-x|
\end{aligned}
$$

With $\varepsilon^{-1}:=\max _{\xi \in \mathbb{R}} \sum_{k=1}^{m}\left|\lambda_{k}^{\prime}(\xi)\right|,\left(\right.$ A.2), $(i),(i i)$ imply $\Psi_{k}[\varphi]^{\prime}>0$, which means that $\Psi_{k}[\varphi]$ is a periodic diffeomorphism..

\footnotetext{
${ }^{1}$ The author is indebted to Prof. Fredenhagen (Hamburg) for a discussion on related ideas of an unpublished joint work he had undertaken together with Prof. D'Antoni [DF].
} 
A closer look at the action of the $\Psi_{k}[\varphi], k<m$, reveals that $\check{\Psi}_{k}[\varphi] \circ \check{\Psi}_{k-1}[\varphi]^{-1}$ is localised in $N_{k}^{\prime} \Subset \mathrm{S}^{1}$ and that $\breve{\Psi}_{m}[\varphi] \circ \breve{\Psi}_{m-1}[\varphi]^{-1}$ is localised in $\check{\varphi}\left(M_{m-1}\right)^{\prime}$. We set: $\widetilde{I}_{k}^{\prime}:=N_{m+1-k}$ for $k=2, \ldots, m$, and $\widetilde{I}_{1}{ }^{\prime}:=\check{\varphi}\left(M_{m-1}\right)$.

We define the localising maps by $\Xi_{k}(\varphi):=\Psi_{m+1-k}[\varphi] \circ \Psi_{m-k}[\varphi]^{-1}, k=$ $1, \ldots, m$. Continuous dependence of $\Xi_{k}(\varphi)$ on $\varphi$ is obvious, $\Psi_{k}[i d]=i d$ yields $\Xi_{k}(i d)=i d$. Finally, with $\Psi_{m}[\varphi]=\varphi$ and $\Psi_{0}[\varphi]=i d$ :

$$
\prod_{k=1}^{m} \Xi_{k}(\varphi)=\Psi_{m}[\varphi] \circ \Psi_{m-1}[\varphi]^{-1} \circ \Psi_{m-1}[\varphi] \circ \ldots \circ \Psi_{0}[\varphi]^{-1}=\varphi \circ i d
$$

\section{Alternative argument for lemma IV.2}

The the net-endomorphism property of $U^{\mathcal{A}}$ can be shown to hold using only the stress-energy tensor $\Theta^{\mathcal{A}}$ and without making any (explicit) reference to the exponentiated representation of Diff $\left(\mathrm{S}^{1}\right)^{\sim}$. We indicate the alternative argument proving lemma IV.2 briefly.

If $\Theta^{\mathcal{A}}$ is smeared with real test functions $f$, the respective closures $\Theta^{\mathcal{A}}(f)^{-}$ are essentially self-adjoint on the WIGHTMAN domain, linear in the test function and fulfill locality in the sense of self-adjoint operators [BSM90]. Let $\Theta^{\mathcal{A}}(f)^{-}$, $f(x)=x$, denote the generator of dilatations in the representations $\Upsilon^{\mathcal{A}}, U^{\mathcal{A}}(\mathrm{cf}$ theorem III.3).

We cover the circle $\mathrm{S}^{1}$ by four proper intervals $I_{ \pm}, I_{\varepsilon}, I_{\delta}$, satisfying $\overline{I_{ \pm}} \subset \mathrm{S}_{ \pm}^{1}$, $-1 \in I_{\varepsilon} \Subset \mathrm{S}^{1},+1 \in I_{\delta} \Subset \mathrm{S}^{1}$, where $I_{\varepsilon}$ and $I_{\delta}$ are taken to be arbitrarily small. We take a partition of unity subordinate to this covering by real test functions and thus gain the corresponding decomposition of $\widetilde{f}$ into test functions with compact support in the respective proper intervals: $\widetilde{f}=f_{+}+f_{-}+f_{\delta}+f_{\varepsilon}$. If one smears $\widetilde{\Theta}^{\mathcal{A}}$ with $f_{+}, f_{-}, f_{\delta}, f_{\varepsilon}$ or a real linear combination, one gets an essentially self-adjoint operator on the WIGHTMAN domain.

According to TrotTER's formula [RS72, theorem VIII.31] we have in the strong operator topology:

$$
\lim _{n \rightarrow \infty}\left(e^{i \frac{t}{n} \Theta^{\mathcal{A}}(f)^{-}} e^{-i \frac{t}{n} \widetilde{\Theta}^{\mathcal{A}}\left(f_{+}+f_{-}\right)^{-}}\right)^{n}=e^{-i t \widetilde{\Theta}^{\mathcal{A}}\left(f_{\delta}\right)^{-}} e^{-i t \widetilde{\Theta}^{\mathcal{A}}\left(f_{\varepsilon}\right)^{-}} .
$$

We introduce a new notation: $g_{\tau}(z):=[d(D(-\tau) z) / d z]^{-1} g(D(-\tau) z)$. Covariance of $\Theta^{\mathcal{A}}$ with respect to dilatations then reads as:

$$
A d_{U^{\mathcal{A}}(D(\tau))}\left(e^{i s \widetilde{\Theta}^{\mathcal{A}}(g)^{-}}\right)=e^{i s \widetilde{\Theta}^{\mathcal{A}}\left(g_{\tau}\right)^{-}} .
$$

One can verify easily by looking at the $n$th step of the sequence in TROTTER's formula that the following holds:

$$
e^{i t \Theta^{\mathcal{A}}(f)^{-}}=e^{-i t \widetilde{\Theta}^{\mathcal{A}}\left(f_{\delta}\right)^{-}} e^{-i t \widetilde{\Theta}^{\mathcal{A}}\left(f_{\varepsilon}\right)^{-}} e^{i t \widetilde{\Theta}^{\mathcal{A}}\left(f_{+}+f_{\delta}+f_{\varepsilon}\right)^{-}} e^{i t \widetilde{\Theta}^{\mathcal{A}}\left(\left(f_{-}+f_{\delta}+f_{\varepsilon}\right)_{-t}\right)^{-}} .
$$


It is elementary to see that the strong convergence of the TROTTER formula entails weak (and hence strong) convergence of the operators involved in equation (A.3). This formula yields for $\bar{I} \subset \mathrm{S}_{+}^{1}, I \Subset \mathrm{S}^{1}$ :

$$
U^{\mathcal{A}}(\tilde{D}(t)) \mathcal{B}(I) U^{\mathcal{A}}(\tilde{D}(t))^{*} \subset \bigcap_{J \supset \overline{\mathrm{S}_{+}^{1}}} \mathcal{B}(J)=\mathcal{B}\left(\mathrm{S}_{+}^{1}\right) .
$$

The latter equality is due to continuity from the outside. The remainder follows as in the proof of lemma IV.2.

\section{Detailed proof of the BMT theorem}

The original proof of [BMT88, theorem 1] (BMT theorem) is very brief and we consider it worthwhile to make available a detailed proof. We give a formulation for the chiral situation, the generalisation to other conformal scenarios is straightforward. The author thanks Prof. D. Buchholz (Göttingen) for mentioning the relation of this theorem to the inner-implementing representation of translations.

Proposition A.6: Assume $\pi$ to be a covariant representation of a chiral conformal theory $\mathcal{B}$ and that there exists a vector $\phi$ in the representation HILBERT space $\mathscr{H}_{\pi}$ which is cyclic for $\pi(\mathcal{B})=\bigvee_{I \Subset \mathrm{S}^{1}} \pi_{I}(\mathcal{B}(I))^{\prime \prime}$. The unitary, strongly continuous representation $U_{\pi}$ of $P S L(2, \mathbb{R})^{\sim}$, which implements conformal covariance of $\pi$, shall be of positive energy.

Then, $\pi$ is locally normal and unitarily equivalent to a representation localised in an arbitrary $I_{0} \Subset \mathrm{S}^{1}$.

Proof: $U_{\pi}$ defines automorphisms of $\pi(\mathcal{B})$ and satisfies the spectrum condition. Thus, there is a representation $V_{\pi(\mathcal{B})}$ of the translations which is inner in $\pi(\mathcal{B})$, satisfies the spectrum condition and implements the translations on $\pi$ [Bor66, Arv74] (cf [BR87, theorem 3.2.46]). The positive generator of $V_{\pi(\mathcal{B})}$ shall be called $H$ and for some $t>0$ we set: $\psi:=e^{-t H} \phi . \psi$ is analytic for $V_{\pi(\mathcal{B})}$ in a full neighbourhood of the identity.

We use the spectral decomposition of $H$ to define $M_{n}:=\int_{0}^{n} d E_{\lambda} e^{\lambda t}$. These operators are contained in $\pi(\mathcal{B})$. Obviously, we have $X \phi=\lim _{n \rightarrow \infty} X M_{n} \psi$ for every $X \in \pi(\mathcal{B})$. This proves that $\psi$ too is cyclic for $\pi(\mathcal{B})$. By the usual ReEHSCHLIEDER argument [Bor68] the vector $\psi$ is cyclic for some $\pi_{I_{\infty}}\left(\mathcal{B}\left(I_{\infty}\right)\right)^{\prime \prime}$ where $I_{\infty}$ is supposed to contain the point $\infty$. This implies, because of locality, that $\psi$ is separating for each $\pi_{I_{0}}\left(\mathcal{B}\left(I_{0}\right)\right)^{\prime \prime}$, if $I_{0}$ and $I_{\infty}$ are disjoint. Covariance of $\pi$ implies now that each $\pi_{I}(\mathcal{B}(I))^{\prime \prime}$ has a separating vector.

Every V.NEUMANN algebra which possesses a separating vector is $\sigma$-finite [Dix81, I.1.4.,Prop. 6] and every $*$-homomorphism from a $\sigma$-finite, properly infinite V.NEUMANN algebra into a $\sigma$-finite V.NEUMANN algebra is automatically normal [Tak79, V.5, Theorem 5.1] (the local algebras $\mathcal{B}(I)$, which are type $I I I_{1}$ 
factors, are $\sigma$-finite and properly infinite). The argument of [BR87, theorem 2.4.24] shows that the representations $\pi_{I}$ are faithful, too. Normality implies the identity $\pi_{I}(\mathcal{B}(I))=\pi_{I}(\mathcal{B}(I))^{\prime \prime}$ and adding faithfulness we get: $\pi_{I}(\mathcal{B}(I)) \cong \mathcal{B}(I)$. Since $\mathcal{B}(I)$ is a type $I I I$ factor this isomorphy is in fact spatial, ie: it may be implemented by a unitary from $\mathscr{H}_{\pi}$ onto $\mathscr{H}$ (eg [Sch67, II.4.6., Theorem]). If we pick a $\mathcal{B}\left(I_{0}\right)$ and denote the unitary implementer of the isomorphism between $\pi_{I}\left(\mathcal{B}\left(I_{0}\right)\right)$ and $\mathcal{B}\left(I_{0}\right)$ by $W$, then $\rho_{\pi}():.=A d_{W}(\pi()$.$) defines a unitarily equivalent,$ localised representation on $\mathscr{H}$.

\section{$5 \quad \operatorname{Diff}_{+}\left(\mathrm{S}^{1}\right)$-Symmetry and $P S L(2, \mathbb{R})$-Covariance}

We deduce covariance with respect to global conformal transformations for all locally normal representations of diffeomorphism covariant chiral components of a factorising conformal theory in $1+1$ dimensions. As an application we prove the statement of [GLW98] saying that the theory of the first conformally covariant derivative of the $U(1)$ current does not contain a stress-energy tensor.

Diffeomorphism covariance of a chiral net $\mathcal{B}$ means that there is a strongly continuous map $\Upsilon_{0}$ from the orientation preserving diffeomorphisms of the circle, Diff $\left(\mathrm{S}^{1}\right)$, into the unitaries on $\mathscr{H}$, the representation space of the vacuum representation of $\mathcal{B}$, implementing a geometric automorphic action $\alpha$ of $\operatorname{Diff}_{+}\left(\mathrm{S}^{1}\right)$ :

$$
\Upsilon_{0}(\phi) \mathcal{B}(I) \Upsilon_{0}(\phi)^{*} \equiv \alpha_{\phi}(\mathcal{B}(I))=\mathcal{B}(\phi(I)), I \Subset \mathrm{S}^{1}, \phi \in \operatorname{Diff}_{+}\left(\mathrm{S}^{1}\right)
$$

If $\phi$ acts trivially on an interval $I^{\prime}$, then $A d_{\Upsilon_{0}(\phi)}$ is to implement the trivial automorphism of $\mathcal{B}\left(I^{\prime}\right)$; such $\phi$ is said to be localised in $I$ and gives rise to a local operator $\Upsilon_{0}(\phi) \in \mathcal{B}(I)$, by HAAG duality of $\mathcal{B}$. $\Upsilon_{0}$ defines a ray representation, as the cocycles $\Upsilon_{0}\left(\phi_{1}\right) \Upsilon_{0}\left(\phi_{2}\right) \Upsilon_{0}\left(\phi_{1} \phi_{2}\right)^{*}$ commute with $\mathcal{B}$ and $\mathcal{B}$ is irreducible. We require $\Upsilon_{0}(i d)=\mathbb{1}$ and $\alpha \uparrow P S L(2, \mathbb{R})$ to be identical to the global conformal covariance of $\mathcal{B}$. Since Diff $\left(\mathrm{S}^{1}\right)$ is a simple group (theorem of EPSTEIN, HeRMan, Thurston, of [Mil84]) the whole representation $\Upsilon_{0}$ is contained in $\mathcal{B}_{\text {uni }}$, the universal $C^{*}$-algebra generated by the local algebras $\mathcal{B}(I), I \Subset \mathrm{S}^{1}$.

In models having a stress-energy tensor, the restricted representation $\Upsilon_{0} \uparrow$ $\operatorname{PSL}(2, \mathbb{R})$ is in fact a representation of $P S L(2, \mathbb{R})$ (cf theorem III.3). The further analysis does not require the answer to the cohomological question whether this may be achieved always by a proper choice of phases for $\Upsilon_{0}$ and we shall, therefore, not concern ourselves with this problem.

We deal with a locally normal representation $\pi$ of $\mathcal{B}$, ie a family of normal representations $\pi_{I}$ of the local algebras $\mathcal{B}(I)$ by bounded operators on a HILBERT space $\mathscr{H}_{\pi}$, which is required to be consistent with isotony: $I \subset J \Rightarrow \pi_{J} \uparrow \mathcal{B}(I)=$ $\pi_{I}$. This family lifts uniquely to a representation $\pi$ of $\mathcal{B}_{\text {uni }}$ and the $\pi_{I}$ are given in terms of the embeddings $\iota_{I}: \mathcal{B}(I) \hookrightarrow \mathcal{B}_{\text {uni }}$ by $\pi_{I}=\pi \circ \iota_{I}$ [Fre90, GL92]. 
It is easy to see, that $\pi \circ \Upsilon_{0}$ implements the automorphic action $\alpha$ and represents the group laws of Diff $\left(\mathrm{S}^{1}\right)$ up to multiplication with operators in the centre of $\pi\left(\mathcal{B}_{\text {uni }}\right)$. The restrictions of $\pi \circ \Upsilon_{0}$ to subgroups of localised diffeomorphisms are weakly and thus strongly continuous by local normality and the local cocycles are phases, since local algebras are factors. We note that $\pi$ is unital because of local normality.

We will now restrict our attention to the subgroup of global conformal transformations, $P S L(2, \mathbb{R})$, and construct a unitary, strongly continuous representation of its universal covering group $P S L(2, \mathbb{R})^{\sim}$ from $\pi \circ \Upsilon_{0} \uparrow P S L(2, \mathbb{R})$. This representation will implement the automorphic action $\alpha$ of $P S L(2, \mathbb{R})$ on $\mathcal{B}$ in the representation $\pi$ and will be inner in the global sense, ie it will be contained in the V.NEUmANN algebra of global observables, $\pi(\mathcal{B}):=\bigvee_{I \Subset S^{1}} \pi_{I}(\mathcal{B}(I))$. The line of argument will be very similar to the one leading to theorem II.14.

We can write every $g \in P S L(2, \mathbb{R})$ in the form $g=T\left(p_{g}\right) D\left(\tau_{g}\right) R\left(t_{g}\right)$, where each term depends continuously on $g$ (IWASAWA decomposition, [GF93, appendix I]). In fact, any $g \in P S L(2, \mathbb{R})$ may be written as a product of four translations and four special conformal transformations, each single of them depending continuously on $g$, if one uses the identities:

$$
\begin{aligned}
D(\tau) & =S\left(-\left(e^{\frac{\tau}{2}}-1\right) e^{-\frac{\tau}{2}}\right) T(1) S\left(e^{\frac{\tau}{2}}-1\right) T\left(-e^{-\frac{\tau}{2}}\right) \\
R(2 t) & =S\left((-1+\cos t)(\sin t)^{-1}\right) T(\sin t) S\left((-1+\cos t)(\sin t)^{-1}\right) .
\end{aligned}
$$

According to lemma A.5, there are continuous, identity preserving localisation maps $\Xi_{j}, j=1, . ., m$, which map a neighbourhood of the identity, $\mathcal{U}_{\varepsilon} \subset \operatorname{Diff}_{+}\left(\mathrm{S}^{1}\right)$, into groups of localised diffeomorphisms such that we have $\prod_{j=1}^{m} \Xi_{j}(\phi)=\phi$, $\phi \in \mathcal{U}_{\varepsilon}$. If we specialise to translations, this means that there is an open interval $I_{\varepsilon}$ containing 0 for which the mapping $t \mapsto \prod_{j} \pi \circ \Upsilon_{0}\left(\Xi_{j}(T(t))\right)$ is unital and strongly continuous. We extend this map to all of $\mathbb{R}$ through a choice of a $\tau \in I_{\varepsilon}$, $\tau>0$, defining $n_{t} \in \mathbb{Z}$ by its properties $t=n_{t} \tau+\left(t-n_{t} \tau\right), t-n_{t} \tau \in[0, \tau[$, and setting

$$
T^{\pi(\mathcal{B})}(t):=\left(\prod_{j} \pi \circ \Upsilon_{0}\left(\Xi_{j}(T(\tau))\right)\right)^{n_{t}} \prod_{j} \pi \circ \Upsilon_{0}\left(\Xi_{j}\left(T\left(t-n_{t} \tau\right)\right)\right)
$$

One can easily check that this is indeed a weakly and thus strongly continuous map into the unitaries on $\mathscr{H}_{\pi}$ by recognising that the mappings involved are continuous and unital $\left(\pi(\mathbb{1})=\mathbb{1}, \Xi_{i}(i d)=i d\right)$.

This procedure applies to the special conformal transformations as well, and we may use the result, the IWASAwA decomposition and (A.5), (A.6) to define for each $g \in P S L(2, \mathbb{R})$ :

$$
\pi^{\mathcal{B}}(g):=\prod_{i=1}^{4} T^{\pi(\mathcal{B})}\left(t_{g}^{(i)}\right) S^{\pi(\mathcal{B})}\left(n_{g}^{(i)}\right), \quad g \in P S L(2, \mathbb{R})
$$


We have $\pi^{\mathcal{B}}(i d)=\mathbb{1}$. The following lemma asserts that the $\pi^{\mathcal{B}}(g)$ define an innerimplementing representation up to a cocycle in the centre of $\pi(\mathcal{B})$. To this end we define operators sensitive to the violation of the group multiplication law: $z^{\mathcal{B}}(g, h):=\pi^{\mathcal{B}}(g) \pi^{\mathcal{B}}(h) \pi^{\mathcal{B}}(g h)^{*}, g, h \in P S L(2, \mathbb{R})$.

Lemma A.7: $\pi^{\mathcal{B}}: g \mapsto \pi^{\mathcal{B}}(g)$ defines a strongly continuous mapping with unitary values in $\pi(\mathcal{B})$. The adjoint action of $\pi^{\mathcal{B}}(g), g \in P S L(2, \mathbb{R})$, on $\pi(\mathcal{B})$ implements the automorphism $\alpha_{g} \cdot z^{\mathcal{B}}:(g, h) \mapsto z^{\mathcal{B}}(g, h)$ defines a strongly continuous 2-cocycle with unitary values in $\pi(\mathcal{B})^{\prime} \cap \pi(\mathcal{B})$.

Proof: Unitarity is obvious. Strong continuity follows since we multiply continuous functions. The implementing property of the $\pi^{\mathcal{B}}(g)$ follows immediately by the decomposition $g=\prod_{i=1}^{4} T\left(t_{g}^{(i)}\right) S\left(s_{g}^{(i)}\right)$, the subsequent decomposition of these into products of localised diffeomorphisms, the definition of $\pi^{\mathcal{B}}(g)$ and the implementation property of the (generalised) ray representation $\pi \circ \Upsilon_{0}$ of $\operatorname{Diff}_{+}\left(\mathrm{S}^{1}\right)$. At this point all but the cocycle properties of $z^{\mathcal{B}}$ follow immediately from its definition. If we look at $\pi^{\mathcal{B}}(f) \pi^{\mathcal{B}}(g) \pi^{\mathcal{B}}(h)$, insert some identities appropriately, we find: $z^{\mathcal{B}}(f, g h) z^{\mathcal{B}}(g, h)=z^{\mathcal{B}}(f, g) z^{\mathcal{B}}(f g, h)$. Even more immediate are the equalities $z^{\mathcal{B}}(i d, g)=z^{\mathcal{B}}(g, i d)=\mathbb{1}$.

We write the abelian V.NEUMANN algebra generated by the cocycle operators $z^{\mathcal{B}}(g, h)$ as follows: $\mathcal{Z}^{\mathcal{B}} \equiv\left\{z^{\mathcal{B}}(g, h), z^{\mathcal{B}}(g, h)^{*} \mid g, h \in P S L(2, \mathbb{R})\right\}^{\prime \prime}$. Obviously $\mathcal{Z}^{\mathcal{B}}$ is contained in the centre of $\pi(\mathcal{B})$. Now we are prepared to realise the construction itself:

Lemma A.8: For every $\tilde{g} \in P S L(2, \mathbb{R})^{\sim}$ there exists a unitary operator $z^{\mathcal{B}}(\tilde{g}) \in$ $\mathcal{Z}^{\mathcal{B}}$ such that

$$
U_{\pi}(\tilde{g}):=z^{\mathcal{B}}(\tilde{g}) \pi^{\mathcal{B}}(\mathrm{p}(\tilde{g}))
$$

defines a unitary, strongly continuous representation, whose adjoint action implements the automorphic action $\alpha \circ \mathrm{p}$ on $\pi(\mathcal{B})$.

Proof: The proof of lemma II.13 applies word for word.

The outcome of the construction presented above proves the main result of this section. It was known already, perhaps not in the present formulation, to D'Antoni And Fredenhagen [DF]; its uniqueness statement is a simple consequence of the fact that $\operatorname{PSL}(2, \mathbb{R})^{\sim}$ is a perfect group (proposition II.9):

Theorem A.9: Let $\mathcal{B}$ be a chiral conformal, diffeomorphism covariant theory. Then any locally normal representation $\pi$ of $\mathcal{B}$ is covariant with respect to the automorphic action of $P S L(2, \mathbb{R})$. The implementing representation may be chosen to be the unique globally $\pi(\mathcal{B})$-inner, implementing representation $U_{\pi}$ of $\operatorname{PSL}(2, \mathbb{R})^{\sim}$. 
The construction given here for diffeomorphism covariant theories is more general than the Borchers-SugawARA construction (section II.2), if these possess locally normal representations which violate positivity of energy. For representations with finite statistical dimension the spectrum condition is always fulfilled because of the theorem we have just derived and results of [BCL98]. For infinite index representations there exists a criterion for strongly additive theories; it was given in [BCL98], too. In presence of the spectrum condition the construction given here and the BORCHERS-SUGAWARA construction agree by uniqueness.

As an application we have the following corollary, which provides an explicit proof for the closing remark of [GLW98] and is an alternative for the proof of proposition VI.3 in the case $n=1$; we make the same assumptions on stressenergy tensors as in section VI,2:

Corollary A.10: There is no stress-energy tensor in the $\Phi^{(1)}$-model.

Proof: Lets assume that there was a stress-energy tensor in this model. Then the FoCK space of $\Phi^{(1)}$ decomposes completely, as representation space of the VIRAsORO algebra, into irreducible highest-weight representations [Kac90, proposition 11.12.c]. Since for fixed $c<1$ there are only finitely many allowed ground states and the energy eigenspaces are finite-dimensional, this decomposition would be finite. For $c<1$, the theory generated by the stress-energy tensor, $\mathcal{B}_{\Theta}$, is completely rational [KL02], a property it would pass on to the theory generated by $\Phi^{(1)}$, denoted $\mathcal{B}_{\Phi^{(1)}}$ [Lon01]. In particular, $\mathcal{B}_{\Phi^{(1)}}$ would be strongly additive, which it is not [Yng94].

The particular shape of Fock space teaches us the following: From the representation theory of the VIRASORO algebra for $\mathrm{c}=1$ [RC85] and for $\mathrm{c}>1$ [Lan88, lemma 2] we learn that the set of vectors $L_{-m_{1}} \ldots L_{-m_{k}} \Omega, m_{1} \geq \ldots \geq m_{k}>1$, at a particular level $\sum_{i} m_{i}$ is linearly independent, ie such a stress-energy tensor would generate a dense set of vectors from the vacuum as does $\Phi^{(1)}$. The same holds for the local quantum theories generated by both fields, $\mathcal{B}_{\Theta}$ and $\mathcal{B}_{\Phi^{(1)}}$, respectively.

By conformal covariance and the BISOGNANO-WICHMANN property for chiral conformal theories the local algebras $\mathcal{B}_{\Theta}(I)$ are modular covariant subalgebras of the local algebras $\mathcal{B}_{\Phi^{(1)}}(I)$. By results of TAKESAKI [Tak72], JonEs [Jon83] and the REEH-SCHLIEDER theorem we know that the projection $e_{\Theta}$ onto $\overline{\mathcal{B}_{\Theta} \Omega}$ completely characterises $\mathcal{B}_{\Theta}$ through $\mathcal{B}_{\Theta}(I)=\left\{e_{\Theta}\right\}^{\prime} \cap \mathcal{B}_{\Phi^{(1)}}(I)$. We have just deduced $e_{\Theta}=\mathbb{1}$, and thus the two local quantum theories coincide. Both fields have to be regarded as different coordinates of the same theory.

The representation of the VIRASORO algebra defined by the commutation relations of the stress-energy tensor integrates to a projective representation of Diff $_{+}\left(\mathrm{S}^{1}\right)[\mathrm{GW} 85]$. A generating set of the local algebras $\mathcal{B}_{\Theta}(I)$ is given by all oneparameter groups $\exp (i t \Theta(f)), \operatorname{supp}(f) \subset I, \Theta(f)$ symmetric, which represent one-parameter subgroups of Diff $+\left(\mathrm{S}^{1}\right)$ [BSM90, Lok94]. This shows diffeomorphism covariance of $\mathcal{B}_{\Theta}$ and, by assumption, of $\mathcal{B}_{\Phi^{(1)}}$. 
By theorem A.9 any locally normal representation of $\mathcal{B}_{\Phi^{(1)}}$ would be covariant, but [GLW98] have given DHR-automorphisms for this model, which are not covariant. This contradicts the assumption. 


\section{Bibliography}

[AGO87] R. Arcuri, J. Gomes, and D. Olive. Conformal subalgebras and symmetric spaces. Nuclear Phys. B285 (1987) 327.

[Ara92] H. Araki. Symmetries in theory of local observables and the choice of the net of local algebras. Rev. Math. Phys. SI 1 (Special Issue) (1992) $1-14$.

[Arv74] W. Arveson. On groups of automorphisms of operator algebras. J. Funct. Anal. 15 (1974) 217-243.

[Bau76] K. Baumann. There are no scalar Lie fields in three or more dimensional space-time. Comm. Math. Phys. 47 (1976) 69-74.

[Bau97] K. Baumann. Unboundedness of Bose fields in a one-dimensional model world. Lett. Math. Phys. 41 (1997) 135-147.

[Bau99] K. Baumann. Bounded Bose fields in $1+1$ dimensions commuting for space- and timelike distances. J. Math. Phys. 40 (1999) 1719-1737.

[BB85] H.-J. Borchers and D. Buchholz. The energy-momentum spectrum in local field theories with broken Lorentz-symmetry. Comm. Math. Phys. 97 (1985) 169-185.

[BB87] F. Bais and P. Bouwknegt. A classification of subgroup truncations of the bosonic strings. Nuclear Phys. B279 (1987) 561.

[BCL98] P. Bertozzini, R. Conti, and R. Longo. Covariant sectors with infinite dimension and positivity of the energy. Comm. Math. Phys. 141 (1998) 471-492.

[BDF87] D. Buchholz, C. D'Antoni, and K. Fredenhagen. The universal structure of local algebras. Comm. Math. Phys. 111 (1987) 123-135.

[BDFS00] D. Buchholz, O. Dreyer, M. Florig, and S. J. Summers. Geometric modular action and spacetime symmetry groups. Rev. Math. Phys. 12 (2000) 475-560. 
[BDL86] D. Buchholz, S. Doplicher, and R. Longo. On Noether's theorem in quantum field theory. Ann. Physics 170 (1986) 1-17.

[BE99] J. Bockenhauer and D. E. Evans. Modular invariants, graphs and $\alpha-$ induction for nets of subfactors II. Comm. Math. Phys. 200 (1999) 57-103.

[BG87] P. Bowcock and P. Goddard. Virasoro algebras with central charge $c<1$. Nuclear Phys. B285 [FS19] (1987) 651-670.

[BGL93] R. Brunetti, D. Guido, and R. Longo. Modular structure and duality in conformal quantum field theory. Comm. Math. Phys. 156 (1993) 201-219.

[BGL95] R. Brunetti, D. Guido, and R. Longo. Group cohomology, modular theory and space-time symmetries. Rev. Math. Phys. 7 (1995) 57-71.

[BH71] K. Bardakci and M. B. Halpern. New dual quark models. Phys. Rev. D3 (1971) 2493.

[BLT75] N. Bogolubov, A. Logunov, and I. Todorov. Introduction to Axiomatic Quantum Field Theory. Benjamin, Reading, MA, 1975.

[BMT88] D. Buchholz, G. Mack, and I. Todorov. The current algebra on the circle as a germ of local field theories. Nuclear-Physics-B, ProceedingsSupplements 5B (1988) 20-56.

[Bor66] H.-J. Borchers. Energy and momentum as observables in quantum field theory. Comm. Math. Phys. 2 (1966) 49-54.

[Bor68] H.-J. Borchers. On the converse of the Reeh-Schlieder theorem. Comm. Math. Phys. 10 (1968) 269-273.

[Bor84] H.-J. Borchers. Translation group and spectrum condition. Comm. Math. Phys. 96 (1984) 1-13.

[Bor87] H.-J. Borchers. On the interplay between spectrum condition and locality in quantum field theory. Contemp. Math. 62 (1987) 143-152.

[Bor92] H.-J. Borchers. The CPT theorem in two-dimensional theories of local observables. Comm. Math. Phys. 143 (1992) 315-332.

[Bor97a] H.-J. Borchers. Half-sided modular inclusions and structure analysis in quantum field theory. In S. Doplicher, R. Longo, J. Roberts, and L. Zsido (eds.), Operator Algebras and Quantum Field Theory. International Press, Cambridge, MA, 1997; pp. 589-608. 
[Bor97b] H.-J. Borchers. On the lattice of subalgebras associated with the principle of half-sided modular inclusion. Lett. Math. Phys. 40 (1997) 371-390.

[Bor98] H.-J. Borchers. Half-sided translations and the type of von Neumann algebras. Lett. Math. Phys. 44 (1998) 283-290.

[Bor00] H.-J. Borchers. On revolutionizing quantum field theory with Tomita's modular theory. J. Math. Phys. 41 (2000) 3604-3673.

[BPZ84] A. Belavin, A. Polyakov, and A. Zamolodchikov. Infinite conformal symmetry in two-dimensional quantum field theory. Nuclear Phys. B241 (1984) 333.

[BR77] A. Barut and R. Rączka. Theory of Group Representations and Applications. PWN - Polish Scientific Publishers, Warszawa, 1977.

[BR87] O. Bratteli and D. Robinson. Operator Algebras and Quantum Statistical Mechnics I. $C^{*}$ - und $W^{*}$-Algebras, Symmetry Groups, Decomposition of States. Springer, New York, 1987, 2nd edition.

[BS93] P. Bouwknegt and K. Schoutens. $\mathcal{W}$-symmetry in conformal field theory. Phys. Rep. 223 (1993) 183-276.

[BSM90] D. Buchholz and H. Schulz-Mirbach. Haag duality in conformal quantum field theory. Rev. Math. Phys. 2 (1990) 105-125.

[Buc74] D. Buchholz. Product states for local algebras. Comm. Math. Phys. 36 (1974) 287-304.

[Buc90] D. Buchholz. Harmonic analysis of local operators. Comm. Math. Phys. 129 (1990) 631-641.

[Buc00] D. Buchholz. Current trends in axiomatic quantum field theory. Lecture Notes in Phys. 558 (2000) 43-64.

[BW75] J. J. Bisognano and E. H. Wichmann. On the duality condition for a hermitian scalar field. J. Math. Phys. 16 (1975) 985-1007.

[BW76] J. J. Bisognano and E. H. Wichmann. On the duality condition for quantum fields. J. Math. Phys. 17 (1976) 303-321.

[BW86] D. Buchholz and E. Wichmann. Causal independence and the energylevel density of states in local quantum field theory. Comm. Math. Phys. 106 (1986) 321-344.

[BZ63] H. Borchers and W. Zimmermann. On the self-adjointness of field operators. Nuovo Cimento XXXI (1963) 1047-1059. 
[Car98] S. Carpi. Absence of subsystems for the Haag-Kastler net generated by the energy-momentum tensor in two-dimensional conformal field theory. Lett. Math. Phys. 45 (1998) 259-267.

[Car99] S. Carpi. Quantum Noether's theorem and conformal field theory: Study of some models. Rev. Math. Phys. 11 (1999) 519-532.

[Car02] S. Carpi. The Virasoro algebra and sectors with infinite statistical dimension, 2002. math.OA/0203027.

[CC] S. Carpi and R. Conti. (In preparation).

[CC01] S. Carpi and R. Conti. Classification of subsystems for local nets with trivial superselection structure. Comm. Math. Phys. 217 (2001) 89-106.

[Con73] A. Connes. Une classification des facteurs de types III. Ann. Sci. École Norm. Sup. (4) 6 (1973) 133-252.

[Con95] R. Conti. On the intrinsic definition of local observables. Lett. Math. Phys. 35 (1995) 237-250.

[Cor89] J. Cornwell. Group Theory in Physics, Volume III: Supersymmetries and Infinite-Dimensional Algebras. Academic Press, London, San Diego, 1989.

[Dab96] C. Daboul. Algebraic proof of the symmetric space theorem. J. Math. Phys. 37 (1996) 3576-3586.

[Dav96] D. Davidson. Endomorphism semigroups and lightlike translations. Lett. Math. Phys. 38 (1996) 77-90.

[DDFL87] C. D'Antoni, S. Doplicher, K. Fredenhagen, and R. Longo. Convergence of local charges and continuity properties of $W^{*}$-inclusions. Comm. Math. Phys. 110 (1987) 325-348.

[DF] C. D'Antoni and K. Fredenhagen. (Private communication).

[DF77] W. Driessler and J. Fröhlich. The reconstruction of local observable algebras from Euclidean Green's functions of a relativistic quantum field theory. Ann. Inst. H. Poincaré A 27 (1977) 221-236.

[DFMS96] P. Di Francesco, P. Mathieu, and D. Sénéchal. Conformal Field Theory. Springer Verlag, Berlin, Heidelberg, New York, 1996.

[DHR69a] S. Doplicher, R. Haag, and J. Roberts. Fields, observables and gauge transformations I. Comm. Math. Phys. 13 (1969) 1-23. 
[DHR69b] S. Doplicher, R. Haag, and J. Roberts. Fields, observables and gauge transformations II. Comm. Math. Phys. 15 (1969) 173-200.

[DHR71] S. Doplicher, R. Haag, and J. Roberts. Local observables and particle statistics I. Comm. Math. Phys. 23 (1971) 199-230.

[DHR74] S. Doplicher, R. Haag, and J. Roberts. Local observables and particle statistics II. Comm. Math. Phys. 35 (1974) 49-85.

[Dix81] J. Dixmier. Von Neumann Algebras. North-Holland, Amsterdam, 1981.

[DL84] S. Doplicher and R. Longo. Standard and split inclusions of von Neumann algebras. Invent. Math. 75 (1984) 493-536.

[DLR01] C. D'Antoni, R. Longo, and F. Radulescu. Conformal nets, maximal temperature and models from free probability. J. Operator Theory 45 (2001) 195-208.

[Dop92] S. Doplicher. Progress and problems in algebraic quantum field theory. In S. Albeverio (ed.), Ideas and methods in quantum and statistical physics, volume 2. Cambridge University Press, Cambridge, 1992; pp. 390-404.

[DR90] S. Doplicher and J. E. Roberts. Why there is a field algebra with a compact gauge group describing the superselection structure in particle physics. Comm. Math. Phys. 131 (1990) 51-107.

[Dri75] W. Driessler. Comments on light-like translations and applications in relativistic quantum field theory. Comm. Math. Phys. 44 (1975) $133-141$.

[DSW86] W. Driessler, S. Summers, and E. Wichmann. On the connection between quantum fields and von Neumann algebras of local operators. Comm. Math. Phys. 105 (1986) 49-84.

[Dyn57a] E. Dynkin. Semisimple subalgebras of semisimple Lie algebras. Amer. Math. Soc. Transl. 6 (1957) 111-244.

[Dyn57b] E. Dynkin. The maximal subgroups of the classical groups. Amer. Math. Soc. Transl. 6 (1957) 245-378.

[EHH93] W. Eholzer, A. Honecker, and R. Hübel. How complete is the classification of $\mathcal{W}$-symmetries ?. Phys. Lett. B 308 (1993) 42-50.

[FJ96] K. Fredenhagen and M. Jörß. Conformal Haag-Kastler nets, pointlike localized fields, and the existence of operator product expansions. Comm. Math. Phys. 176 (1996) 541-554. 
[FK80] I. Frenkel and V. Kac. Basic representations of affine Lie algebras and dual resonance models. Invent. Math. 62 (1980) 23-66.

[FL80] W. Fischer and I. Lieb. Funktionentheorie. Vieweg, Wiesbaden, 1980.

[Flo98] M. Florig. On Borchers' theorem. Lett. Math. Phys. 46 (1998) 289293.

[FQS84] D. Friedan, Z. Qiu, and S. Shenker. Conformal invariance, unitarity, and critical exponents in two dimensions. Phys. Rev. Lett. 52 (1984) 1575 .

[FQS85a] D. Friedan, Z. Qiu, and S. Shenker. Conformal invariance, unitarity and two dimensional critical exponents. In Lepowsky et al. [LMS85], pp. 419-449.

[FQS85b] D. Friedan, Z. Qiu, and S. Shenker. Superconformal invariance in two dimensions and the tricritical Ising model. Phys. Lett. 151 B (1985) 37-43.

[FQS86] D. Friedan, Z. Qiu, and S. Shenker. Details of the non-unitarity proof for highest weight representations of the Virasoro algebra. Comm. Math. Phys. 107 (1986) 535-542.

[Fre90] K. Fredenhagen. Generalizations of the theory of superselection sectors. In Kastler [Kas90].

[Fre91] K. Fredenhagen. On the general theory of quantized fields. In Proceedings of the IAMP conference Leipzig 1991. 1991.

[Fri78] A. Frigerio. Stationary states of quantum dynamical semigroups. Comm. Math. Phys. 63 (1978) 269-276.

[Frö77] J. Fröhlich. Application of commutator theorems to the integration of representations of Lie algebras and commutation relations. Comm. Math. Phys. 54 (1977) 135-150.

[FRS89] K. Fredenhagen, K.-H. Rehren, and B. Schroer. Superselection sectors with braid group statistics and exchange algebras I. Comm. Math. Phys. 125 (1989) 201-226.

[FRS92] K. Fredenhagen, K.-H. Rehren, and B. Schroer. Superselection sectors with braid group statistics and exchange algebras II. Rev. Math. Phys. SI 1 (Special Issue) (1992) 113-157.

[FST89] P. Furlan, G. Sotkov, and I. Todorov. Two-dimensional conformal quantum field theory. Riv. Nuovo Cim. 12 (1989) 1-203. 
[Fuc92] J. Fuchs. Affine Lie Groups and Quantum Groups. Cambridge University Press, 1992.

[Fuc94] J. Fuchs. Fusion rules in conformal field theory. Fortschr. Phys. 42 (1994) 1-48.

[Fuc97] J. Fuchs. Lectures on conformal field theory and Kac-Moody algebras. In Horvath [Hor97].

[GF93] F. Gabbiani and J. Fröhlich. Operator algebras and conformal field theory. Comm. Math. Phys. 155 (1993) 569-640.

[GKO85] P. Goddard, A. Kent, and D. Olive. Virasoro algebras and coset space models. Phys. Lett. 152 B (1985) 88-92.

[GKO86] P. Goddard, A. Kent, and D. Olive. Unitary representations of the Virasoro and super-Virasoro algebras. Comm. Math. Phys. 103 (1986) $105-119$.

[GL92] D. Guido and R. Longo. Relativistic invariance and charge conjugation in quantum field theory. Comm. Math. Phys. 148 (1992) 521551.

[GL96] D. Guido and R. Longo. The conformal spin and statistics theorem. Comm. Math. Phys. 181 (1996) 11-35.

[GLW98] D. Guido, R. Longo, and H.-W. Wiesbrock. Extensions of conformal nets and superselection sectors. Comm. Math. Phys. 192 (1998) 217244.

[GNO85] P. Goddard, W. Nahm, and D. Olive. Symmetric spaces, Sugawara's energy momentum tensor in two dimensions and free fermions. Phys. Lett. B160 (1985) 111-116.

[GO86] P. Goddard and D. Olive. Kac-Moody and Virasoro algebras in relation to quantum physics. Internat. J. Modern Phys. A 1 (1986) 303-414.

[Gom86] F. Gomes. The triviality of representations of the Virasoro algebra with vanishing central element and $L_{0}$ positive. Phys. Lett. 171B (1986) 75.

[GR00] M. Grott and K.-H. Rehren. On a class of bounded Bose fields. Lett. Math. Phys. 53 (2000) 167-179.

[Gri93] D. Grigore. The projective unitary irreducible representations of the Poincaré group in $(1+2)$-dimensions. J. Math. Phys. 34 (1993) 4127-4189. 
[GSW87] M. Green, J. Schwarz, and E. Witten. Superstring theory, Volume 1: Introduction. Cambridge University Press, Cambridge, UK, 1987.

[GW84] R. Goodman and N. Wallach. Structure and unitary cocycle representations of loop groups and the group of diffeomorphisms of the circle. J. Reine Angew. Math. 347 (1984) 69-222.

[GW85] R. Goodman and N. Wallach. Projective unitary positive-energy representations of Diff( $\left.S^{1}\right)$. J. Funct. Anal. 63 (1985) 299-321.

[Haa87] U. Haagerup. Connes' bicentralizer problem and uniqueness of the injective factor of type $I I I_{1}$. Acta Math. 158 (1987) 95-148.

[Haa92] R. Haag. Local Quantum Physics: Fields, Particles, Algebras. Springer, Berlin, Heidelberg, 1992.

[HK64] R. Haag and D. Kastler. An algebraic approach to quantum field theory. J. Math. Phys. 5 (1964) 848-861.

[HKOC96] M. B. Halpern, E. Kiritsis, N. A. Obers, and K. Clubok. Irrational conformal field theory. Phys. Rep. 265 (1996) 1-138.

[HL82] P. D. Hislop and R. Longo. Modular structure of the local algebras associated with the free massless scalar field theory. Comm. Math. Phys. 84 (1982) 71-85.

[HN91] J. Hilgert and K.-H. Neeb. Lie-Gruppen und Lie-Algebren. Vieweg, Braunschweig, 1991.

[Hor97] Z. Horvath (ed.). Conformal Field Theories and Integrable Models, volume 498 of Lecture Notes in Phys.. Springer, Berlin, 1997.

[Jac79] N. Jacobson. Lie Algebras. Dover Publications, New York, 1979.

[Jon83] V. Jones. Index for subfactors. Invent. Math. 72 (1983) 1-25.

[Jör96] M. Jörß. Conformal quantum field theory: From Haag-Kastler nets to Wightman fields. Ph.D. thesis, Universität Hamburg, 1996. DESY 96-136, KEK 96-10-219.

[Jos65] R. Jost. The General Theory of Quantized Fields, volume IV of Lectures in Applied Mathematics. American Mathematical Society, Providence, RI, 1965.

[Kac90] V. Kac. Infinite dimensional Lie algebras. Cambridge University Press, 1990, 3rd edition. 
[Kas90] D. Kastler (ed.). The Algebraic Theory of Superselection Sectors, Introduction and Recent Results. World Scientific, Singapore, 1990. Proceedings of the 1989 Palermo Conference.

[KK92] R. Kadison and D. Kastler. Cohomological aspects and relative separability of finite Jones index factors. Nachr. Akad. Wissensch. Göttingen Jahrgang 1992 (4) 95-105.

[KL02] Y. Kawahigashi and R. Longo. Classification of local conformal nets. Case $c<1$. math-ph/0201015, 2002.

[KLM01] Y. Kawahigashi, R. Longo, and M. Müger. Multi-interval subfactors and modularity of representations in conformal field theory. Comm. Math. Phys. 219 (2001) 631-669.

[KMR90] D. Kastler, M. Mebkhout, and K.-H. Rehren. Introduction to the algebraic theory of superselection sectors. In Kastler [Kas90], pp. $113-214$.

[KNS88] V. G. Kač and M. Niculescu Sanielevici. Decompositions of representations of exceptional affine algebras with respect to conformal subalgebras. Phys. Rev. D 37 (1988) 2231-2237.

[Kös02] S. Köster. Conformal transformations as observables. Lett. Math. Phys. 61 (2002) 187-198.

[Kös03a] S. Köster. Absence of stress energy tensor in CFT $T_{2}$ models, 2003. math-ph/0303053.

[Kös03b] S. Köster. Conformal covariance subalgebras, 2003. hep-th/0303201.

[Kös03c] S. Köster. Local nature of coset models, 2003. math-ph/0303054.

[KP84] V. Kac and D. Peterson. Infinite-dimensional Lie algebras, theta functions and modular forms. Adv. Math. 53 (1984) 125-264.

[KR67] R. Kadison and J. Ringrose. Derivations and automorphisms of operator algebras. Comm. Math. Phys. 4 (1967) 32-63.

[KR83] R. Kadison and J. Ringrose. Fundamentals of the Theory of Operator Algebras, Vol. I: Elementary Theory. Academic Press, New York, 1983.

[KR86] R. Kadison and J. Ringrose. Fundamentals of the Theory of Operator Algebras, Vol. II: Advanced Theory. Academic Press, Orlando, 1986. 
[KW88] V. Kac and M. Wakimoto. Modular and conformal invariance constraints in representation theory of affine algebras. Adv. Math. 70 (1988) 156-236.

[KW01] R. Kähler and H.-W. Wiesbrock. Modular theory and the reconstruction of four-dimensional quantum field theories. J. Math. Phys. 42 (2001) 74-86.

[Lan76] E. C. Lance. Ergodic theorems for convex sets and operator algebras. Invent. Math. 37 (1976) 201-214.

[Lan88] R. P. Langlands. On unitary representations of the Virasoro algebra. In S. N. Kass (ed.), Infinite-Dimensional Lie Algebras and Their Applications. World Scientific, Singapore, New Jersey, Hong Kong, 1988; pp. 141-159.

[LM75] M. Lüscher and G. Mack. Global conformal invariance in quantum field theory. Comm. Math. Phys. 41 (1975) 203-234.

[LM76] M. Lüscher and G. Mack. The energy momentum tensor of critical quantum field theories in $1+1$ dimensions, 1976. Unpublished manuscript, Universität Hamburg.

[LMS85] J. Lepowsky, S. Mandelstam, and I. Singer (eds.). Vertex Operators in Mathematics and Physics, volume 3 of Mathematical Sciences Research Institute Publications. Springer Verlag, New York, Berlin, Heidelberg, Tokyo, 1985.

[Lok94] T. M. Loke. Operator algebras and conformal field theory of the discrete series representations of Diff( $\left(S^{1}\right)$. Ph.D. thesis, University of Cambridge, 1994.

[Lon87] R. Longo. Simple injective subfactors. Adv. Math. 63 (1987) 152-171.

[Lon89] R. Longo. Index of subfactors and statistics of quantum fields I. Comm. Math. Phys. 126 (1989) 217-247.

[Lon90] R. Longo. Index of subfactors and statistics of quantum fields, II. Correspondences, braid group statistics and Jones polynomial. Comm. Math. Phys. 130 (1990) 285-309.

[Lon94] R. Longo. A duality for Hopf algebras and for subfactors. I. Comm. Math. Phys. 159 (1994) 133-150.

[Lon97] R. Longo. An analogue of the Kac-Wakimoto formula and black hole conditional entropy. Comm. Math. Phys. 186 (1997) 451-479. 
[Lon01] R. Longo. Conformal subnets and intermediate subfactors. math.OA/0102196, 2001.

[LR95] R. Longo and K.-H. Rehren. Nets of subfactors. Rev. Math. Phys. 7 (1995) 567-597.

[LRT78] P. Leyland, J. Roberts, and D. Testard. Duality for quantum free fields, 1978. CNRS Marseille 78/P.1016, KEK 79-1-157.

[Mac77] G. Mack. All unitary ray representations of the conformal group $S U(2,2)$ with positive energy. Comm. Math. Phys. 55 (1977) 1-28.

[Mac88] G. Mack. Introduction to conformal invariant quantum field theories in two and more dimensions. In G. t'Hooft et al. (ed.), Nonperturbative Quantum Field Theory. Plenum Press, New York, 1988; pp. 353-383. Cargèse 1987 proceedings.

[Mil84] J. Milnor. Remarks on infinite-dimensional Lie groups. In B. DeWitt and R. Stora (eds.), Relativité, groupes et topologie II/ Relativity, groups and topology II. Elsevier Science Publishers B.V, 1984; pp. 1007-1057. Les Houches, Session XL, 1983.

[Mos94] M. Moskowitz. On the surjectivity of the exponential map for certain Lie groups. Annali di Matematica pura ed applicata (serie IV.) 166 (1994) 129-143.

[Mos97] M. Moskowitz. Correction and addenda to: On the surjectivity of the exponential map for certain Lie groups. Annali di Matematica pura ed applicata (serie IV.) 173 (1997) 351-358.

[MP81] W. McKay and J. Patera. Tables of Dimensions, Indices, and Branching Rules for Representations of Simple Lie Algebras. Marcel Dekker, New York, Basel, 1981.

[Müg02] M. Müger. Research summary (preliminary), September 21, 2002. Universiteit Utrecht, the Netherlands.

[Nel59] E. Nelson. Analytic vectors. Ann. of Math.(2) 70 (1959) 572-615.

[Pet83] D. Petz. Ergodic theorems in von Neumann algebras. Acta Sci. Math. (Szeged) 46 (1983) 329-343.

[P] Platon. Politeia. Insel Verlag, Frankfurt am Main, Leipzig, 1991.

[PP86] M. Pimsner and S. Popa. Entropy and index for subfactors. Ann. Sci. École Norm. Sup. (4) 19 (1986) 57-106. 
[PS86] A. Pressley and G. Segal. Loop Groups. Clarendon Press, Oxford, 1986.

[RC85] A. Rocha-Caridi. Vacuum vector representations of the Virasoro algebra. In Lepowsky et al. [LMS85], pp. 451-473.

[Ree88] H. Reeh. A remark concerning canonical commutation relations. J. Math. Phys. 29 (1988) 1535-1536.

[Reh90] K.-H. Rehren. Braid group statistics and their superselection rules. In Kastler [Kas90], pp. 333-355.

[Reh94a] K.-H. Rehren. A new view of the Virasoro algebra. Lett. Math. Phys. 30 (1994) 125-130.

[Reh94b] K.-H. Rehren. Subfactors and coset models. In H.-D. Doebner, Y. Dobrev, and A. Ushveridze (eds.), Generalized Symmetries in Physics. World Scientific, Singapore, 1994; pp. 338-356.

[Reh95] K.-H. Rehren. On the range of the index of subfactors. J. Funct. Anal. 134 (1995) 183-193.

[Reh97] K.-H. Rehren. Bounded Bose fields. Lett. Math. Phys. 40 (1997) 299-306.

[Reh00] K.-H. Rehren. Chiral observables and modular invariants. Comm. Math. Phys. 208 (2000) 689-712.

[Reh01] K.-H. Rehren. Locality and modular invariance in 2d conformal qft. In R. Longo (ed.), Mathematical Physics in Mathematics and Physics: Quantum and Operator Algebraic Aspects, volume 30 of Fields Institute Communications. American Mathematical Society, Providence, RI, 2001; pp. 341-354. Siena 2000 Proceedings.

[Rob74] J. E. Roberts. Some applications of dilatation invariance to structural questions in the theory of local observables. Comm. Math. Phys. 37 (1974) 273-286.

[Rob76] J. E. Roberts. Local cohomology and superselection structure. Comm. Math. Phys. 51 (1976) 107-119.

[RS61] H. Reeh and S. Schlieder. Bemerkungen zur Unitäräquivalenz von Lorentzinvarianten Feldern. Nuovo Cimento XXII (1961) 1051-1068.

[RS72] M. Reed and B. Simon. Methods of Modern Mathematical Physics I: Functional Analysis. Academic Press, New York, London, 1972. 
[RS75] M. Reed and B. Simon. Methods of Modern Mathematical Physics II: Fourier Analysis and Self-Adjointness. Academic Press, New York, London, 1975.

[RST96] K.-H. Rehren, Y. Stanev, and I. Todorov. Characterizing invariants for local extensions of current algebras. Comm. Math. Phys. 174 (1996) 605-633.

[Sch67] J. T. Schwartz. $W^{*}$-Algebras. Gordon and Breach, New York, London, Paris, 1967.

[Sch74] B. Schoenberg. Elliptic Modular Functions. Springer-Verlag, Berlin, Heidelberg, New York, 1974.

[Sch95] M. Schottenloher. Eine mathematische Einführung in die konforme Feldtheorie. Hamburger Beiträge zur Mathematik aus dem mathematischen Seminar Heft 38 (1995) 1-82.

[Sch97] A. U. Schmidt. Algebraische und konforme Quantenfeldtheorie. Logos Verlag, Berlin, 1997.

[Seg71] I. Segal. Causally oriented manifolds and groups. Bull. Amer. Math. Soc. 77 (1971) 958-959.

[Sim68] D. Simms. Lie Groups and Quantum Mechanics, volume 52 of Lecture Notes in Math.. Springer-Verlag, Berlin, Heidelberg, New York, 1968.

[Sta95] C. P. Staszkiewicz. Die lokale Struktur abelscher Stromalgebren auf dem Kreis. Ph.D. thesis, FU Berlin, 1995.

[Str81] S. Stratila. Modular Theory in Operator Algebras. Editura Academiei/ Abacus Press, Bucuresti, Romania/ Tunbridge Wells, Kent, England, 1981.

[Sug68] H. Sugawara. A field theory of currents. Phys. Rev. 170 (1968) 1659-1662.

[Sum90] S. Summers. On the independence of local algebras in quantum field theory. Rev. Math. Phys. 2 (1990) 201-247.

[SW64] R. Streater and A. Wightman. PCT, Spin and Statistics, and All That. W.A. Benjamin, New York, Amsterdam, 1964.

[SW86] A. Schellekens and N. Warner. Conformal subalgebras of Kac-Moody algebras. Phys. Rev. D 34 (1986) 3092-3096. 
[SZ79] S. Stratila and L. Zsido. Lectures on von Neumann Algebras. Editura Academiei/ Abacus Press, Bucuresti, Romania/ Tunbridge Wells, Kent, England, 1979.

[Tak70] M. Takesaki. Tomita's Theory of Modular Hilbert Algebras and Its Applications, volume 128 of Lecture Notes in Math.. Springer, New York, 1970.

[Tak72] M. Takesaki. Conditional expectations in von Neumann algebras. J. Funct. Analysis 9 (1972) 306-321.

[Tak79] M. Takesaki. Theory of Operator Algebras I. Springer Verlag, New York, 1979.

[Tit39] E. Titchmarsh. The Theory of Functions. Oxford University Press, London, 1939, 2nd edition.

[TL97] V. Toledano Laredo. Fusion of positive energy representations of $\operatorname{LSpin}_{2 n}$. Ph.D. thesis, University of Cambridge, 1997.

[TL99] V. Toledano Laredo. Integrating unitary representations of infinitedimensional Lie groups. J. Funct. Anal. 161 (1999) 478-508.

[Tod65] I. Todorov. Der axiomatische Zugang zur Quantenfeldtheorie. Fortschr. Phys. 13 (1965) 649-700.

[Tod82] I. Todorov. Conformal invariance in (gauge) quantum field theory. In R. Schrader (ed.), VI. International Congress on Mathematical Physics, Berlin, 1981, volume 153 of Lecture Notes in Phys.. Springer, Berlin, Heidelberg, 1982; pp. 319-323.

[Tod94] I. Todorov. What are we learning from 2-dimensional conformal models?. Ben-Gurion University of the Negev Press, 1994; pp. 160-176.

[Tod01] I. Todorov. Two dimensional conformal field theory and beyond. Lessons from a continuing fashion. Lett. Math. Phys. 56 (2001) 151161.

[Ver88] E. Verlinde. Fusion rules and modular transformations in $2 d$ conformal field theory. Nuclear Phys. B300 (1988) 360.

[Was98] A. Wassermann. Operator algebras and conformal field theory III. Fusion of positive energy representations of $L S U(N)$ using bounded operators. Invent. Math. 133 (1998) 467-538.

[Wat97] G. M. Watts. $\mathcal{W}$-algebras and their representations. In Horvath [Hor97], pp. 55-84. 
[WG65] A. Wightman and L. Gårding. Fields as operator-valued distributions in relativistic quantum theory. Arkiv för Fysik 28 (1965) 129-184.

[Wie92] H.-W. Wiesbrock. A comment on a recent work of Borchers. Lett. Math. Phys. 25 (1992) 157-159.

[Wig56] A. Wightman. Quantum field theory in terms of vacuum expectation values. Phys. Rev. 101 (1956) 860-866.

[Xu99] F. Xu. Algebraic coset conformal field theories II. Publ. Res. Inst. Math. Sci. 35 (1999) 795-824.

[Xu00a] F. Xu. Algebraic coset conformal field theories. Comm. Math. Phys. 211 (2000) 1-43.

[Xu00b] F. Xu. Jones-Wassermann subfactors for disconnected intervals. Commun. Contemp. Math. 2 (2000) 307-347.

[Xu01] F. Xu. On a conjecture of Kac-Wakimoto. Publ. Res. Inst. Math. Sci. 37 (2001) 165-190.

[Xu02] F. Xu. On the equivalence of certain coset conformal field theories. Comm. Math. Phys. 228 (2002) 257-279.

[Xu03] F. Xu. Strong additivity and conformal nets, 2003. math.QA/0303266.

[Yng94] J. Yngvason. A note on essential duality. Lett. Math. Phys. 31 (1994) 127-141.

[Zim84] R. J. Zimmer. Ergodic Theory and Semisimple Groups. Birkhäuser, Boston, Basel, Stuttgart, 1984. 


\section{Frequently used symbols}

Most of the notation in this dissertation is in wide-spread use. Therefore, we only give a list of frequently occurring symbols of special importance.

Symbol Description Reference

$T, D, S, R$ one-parameter groups in $\operatorname{PSL}(2, \mathbb{R})$

section II 1.1

$\tilde{T}, \tilde{D}, \tilde{S}, \tilde{R}$ one-parameter groups in $\operatorname{PSL}(2, \mathbb{R})^{\sim}$

section II.1.1

$P, K$

generators of one-parameter groups

section II.1.1

$L_{0}$

conformal Hamiltonian

section II 1.1

$I \Subset \mathrm{S}^{1}, I^{\prime}$

proper interval in $\mathrm{S}^{1}$, its causal complement

$\mathrm{S}_{+}^{1}, \mathrm{~S}_{-}^{1}$

upper and lower half-circle

$\mathbb{C}_{1}$

phases in $\mathbb{C}$

section II 1.1

section II 1.1

section II.1.1

$\mathcal{A} \subset \mathcal{B} \quad$ chiral subnet

page 10

$\mathcal{A}$

net of subalgebras \& its global algebra

pages 10,11

$e_{\mathcal{A}}$ cyclic projection of subnet $\mathcal{A} \subset \mathcal{B}$

page 17

$U^{\mathcal{A}}, U^{\mathcal{A}^{\prime}}$

inner implementations for $\mathcal{A}, \mathcal{A}^{\prime}$

section II.2

$\mathcal{A} \subset \mathcal{C} \subset \mathcal{B} \quad$ Coset pair

page 11

$\mathcal{A} \otimes \mathcal{C}$

vacuum representation of a COSET pair

page 19

$\mathcal{C}_{I}$ local relative commutant

page 11

$\mathcal{C}_{\text {max }}$

maximal Coset model

page 11

$\mathcal{A}_{\text {max }}$

maximal covariance extension of $\mathcal{A} \subset \mathcal{B}$

page 34

$\mathcal{L} G_{k} \quad$ model of loop group $\mathcal{L} G$ at level $k$

page 51

$\mathcal{V} i r_{c}$

model of a stress-energy tensor

page 51

$\operatorname{Diff}_{I}\left(\mathrm{~S}^{1}\right)$

subgroup of localised diffeomorphisms

page 54

$\Phi(f)^{-}$

closure of smeared quantum field $\Phi(f)$

$\widetilde{f}, \widehat{g}$

transformations on test functions

page 45 


\section{Danksagungen}

Ich möchte Herrn Professor K.-H. Rehren für die Gelegenheit danken, eine interessante Fragestellung der mathematischen Physik zu untersuchen, für viele nützliche Diskussionen, die wir miteinander hatten, und für seine beständige und inspirierende Unterstützung. Ich hoffe, dass diese Arbeit seiner Betreuung Ehre macht.

Herrn Professor D. Buchholz möchte ich Dank sagen für stete Ermunterungen und für seine Bereitschaft, als Referent zur Verfügung zu stehen.

Weiterhin danke ich dem Evangelischen Studienwerk Villigst für seine Unterstützung durch ein Promotionsstipendium im Rahmen des Förderschwerpunktes „Wechselwirkung“ und der Berliner-Ungewitter-Stiftung für die Gewährung von Reisemitteln.

Meine Arbeit und ich verdanken allen Angehörigen des Institutes für Theoretische Physik der Universität Göttingen und dem guten Arbeitsklima gerade in der Arbeitsgruppe „Quantenfeldtheorie und Statistische Mechanik“ viele Anregungen und unverzichtbare Unterstützung.

Schließlich wären all diejenigen zu nennen, die mich in den Stand gesetzt und im Stande erhalten haben, diese Arbeit zu verfertigen. Ich kann sie hier gar nicht alle nennen und ihnen an dieser Stelle angemessen danken, vor allem denjenigen nicht, denen ich besonders viel zu verdanken habe - sie werden es wissen. 



\section{Lebenslauf}

\section{Sören Köster}

wurde geboren am 2. November 1968 in Aachen

und ist seitdem deutscher Staatsangehöriger.

Juni 1988 Abitur an der Johannes-Brahms-Schule in Pinneberg

Oktober 1990 - $\quad$ Studium der Physik an der Universität Hamburg

September 1993

August $1992 \quad$ Vordiplom Physik

Oktober 1993 - "Advanced course M.Sc. in elementary particle theory" September 1994 an der University of Durham (UK)

Dezember $1994 \quad$ Verleihung M.Sc.

Oktober 1994 - $\quad$ Studium der Physik an der Universität Hamburg

September 1997

April 1998 - $\quad$ Aufbaustudium Physik an der Georg-August-Universität Götingen,

März 2003 Bearbeitung des Promotionsthemas

„Struktur von Coset-Theorien“

unter der Betreuung von Prof. K.-H. Rehren. 